\title{
A Summary of Cyclic Lateral Load Tests on Rectangular Reinforced Concrete Columns
}

Building and Fire Research Laboratory

National Institute of Standards and Technology

Gaithersburg, Maryland 20899

QC

100

.456

N0.5984

1997

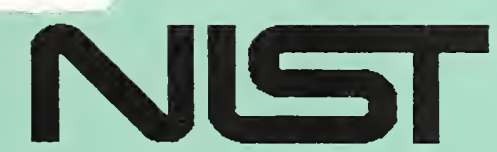

United States Department of Commerce

Technology Administration

National Institute of Standards and Technology 



\section{A Summary of Cyclic Lateral Load Tests on Rectangular Reinforced Concrete Columns}

January 1997

Andrew W. Taylor, Cynthia Kuo, Kevin Wellenius, Duke Chung

Building and Fire Research Laboratory

National Institute of Standards and Technology

Gaithersburg, Maryland 20899

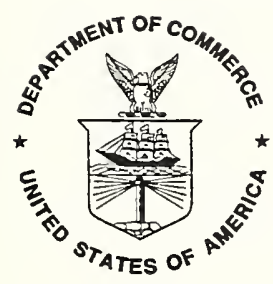

U.S. Department of Commerce

William Daley, Secretary

Technology Administration

Mary L. Good, Under Secretary for Technology

National Institute of Standards and Technology

Arati A. Prabhakar, Director 


\begin{abstract}
Data are summarized from 107 laboratory tests on reinforced concrete columns with rectangular cross sections. In these tests the axial load on the column was held nearly constant while cyclic lateral loads were applied to cause column deformations in the inelastic range. The data in this report were obtained from test programs in the United States, Canada, New Zealand, and Japan. Synopses of each test program are presented and the geometric and material properties of each column test specimen are summarized. Plots of lateral load vs. lateral deflection are also shown for each specimen. The lateral load-lateral deflection histories for all specimens are presented in digital format on a computer disk. Much of this data has only been available in analog format, and is presented for the first time in digital format in this report.
\end{abstract}

KEYWORDS: building technology; cyclic lateral loads; digital data base; earthquake engineering; experiments; inelastic behavior; rectangular reinforcement; reinforced concrete columns 


\section{ACKNOWLEDGMENTS}

We would like to thank the many researchers who provided reports and data to be included in this summary. In a number of cases we requested authors to retrieve data from experiments completed several years before, and they graciously searched their archives for the information we requested. This project was funded by the Federal Highway Administration, the National Center for Earthquake Engineering Research, and the California Department of Transportation. The authors gratefully acknowledge this support. 


\section{CONTENTS}

Chapter 1: Introduction 1

Chapter 2: Descriptions of Cyclic Lateral Load Tests on Spiral Reinforced Columns 3

A. Tests Conducted in New Zealand

1979 Gill

1981 Ang, Priestley and Park

1986 Soesianawati, Park and Priestley 7

1986 Zahn, Park and Priestley 8

1989 Watson and Park 9

1990 Tanaka and Park 10

1990 Park and Paulay 12

B. Tests Conducted in Japan

1982 Arakawa, Arai, Egashira and Fujita 13

1982 Nagasaka 14

1984 Ohno and Nishioka 15

1985 Ohue, Morimoto, Fujii and Morita 16

1985 Zhou, Higashi, Jiang and Shimizu 17

1986 Imai and Yamamoto 18

1987 Zhou, Satoh, Jiang, Ono and Shimizu 19

1987 Kanda, Shirai, Adachi and Sato 21

1989 Arakawa, Arai, Mizoguchi and Yoshida 23

1989 Muguruma, Watanabe and Komuro 24

1989 Ono, Shirai, Adachi and Sakamaki 26

1990 Sakai, Hibi, Otani and Aoyama 27

1991 Amitsu, Shirai, Adachi and Ono 29

C. Tests Conducted in North America

1973 Wight and Sozen $\quad 30$

1975 Atalay and Penzien $\quad 32$

1982 Umehara and Jirsa 34

1985 Bett, Klingner and Jirsa 36

1988 Azizinamini, Johal, Hanson, Musser and Corley 37

1989 Saatcioglu and Ozcebe $\quad 38$

Chapter 3: Plots of Digitized Lateral Load-Lateral Deflection Data 40

Chapter 4: Digital Data Files $\quad$. $\quad 95$ 


\section{Chapter 1: Introduction}

The purpose of this document is to summarize and make available for general use a substantial body of experimental data from cyclic lateral load tests on rectangular reinforced concrete columns. These data were collected, digitized and summarized as part of a larger study of the seismic performance of bridge piers ${ }^{1}$, conducted in the Building and Fire Research Laboratory of the National Institute of Standards and Technology. It is hoped that this data will be useful to other researchers, especially for the calibration of damage models.

Lateral force-lateral deflection data were obtained from 107 column tests conducted by researchers in the United States, Canada, New Zealand and Japan. While the data set is not exhaustive, it represents a significant portion of the available test data.

In some cases the authors were able to obtain digital lateral force-lateral deflection data directly from the researchers who performed the column tests. In the majority of cases, however, precision photo-enlargements of lateral force-lateral deflection plots were prepared from report figures. These enlargements were then digitized manually using a digitizing tablet. Thus, the accuracy of the data was limited by the accuracy of the published figures.

Not every test from every test series was reported in this summary. For some specimens analog plots were either not available or were not clear enough to be used for digitization. Only data from specimens with a constant axial compressive load (not a fluctuating, tensile, or zero axial load) were digitized. Furthermore, only data from specimens in which the lateral load was applied along one of the principal axes of the cross section (not along the diagonal of the cross section or along a bi-direction loading path) were digitized.

The data are reported in Chapters 2 through 4 of this report. In Chapter 2 a summary of each test program is given. This includes bibliographic citations, a brief description of the test program, loading conditions, scale, material properties, geometry, and so on. The names of digital files containing lateral force-lateral deflection data are also given. In Chapter 3 plots of lateral force vs. lateral deflection are presented for all 107 specimens. Finally, in Chapter 4, the digital test data are presented by means of computer disks.

\footnotetext{
${ }^{1}$ Stone, William C.; and Taylor, Andrew W., "Seismic Performance of Circular Bridge Columns Designed in Accordance With AASHTO/CALTRANS Standards," NIST Building Science Series 170, Structures Division, Building and Fire Research Laboratory, National Institute of Standards and Technology, Gaithersburg, Maryland, 20899, February 1993.
} 
$-2-$ 


\section{Chapter 2: Description of Cyclic Lateral Load Tests on Rectangular Reinforced Concrete Columns}

Three types of specimens were tested in the experimental programs described in this report: simple cantilever specimens (Figure 1); double-ended specimens (Figure 2); and double curvature specimens (Figure 3). To present the data from these three types of specimens in a uniform format, all data is reported in terms of an equivalent simple cantilever column. Thus, for cantilever specimens (Figure 1) the forces and deflections reported are simply the same as those reported by the original researchers. For double ended specimens (Figure 2) the lateral forces reported are onehalf the forces applied to the central stub of the specimen, and the lateral deflections reported are the lateral deflections of the central stub relative to the two fixed end points. For double curvature specimens (Figure 3) the lateral forces reported are those applied to each end beam of the specimen, and the lateral deflections reported are one-half the relative lateral displacements of the two end beams. The reported length " $L$ " of each specimen is shown in Figures 1 to 3.

All data shown in the tables of this chapter were those reported by the researchers performing the original study, unless noted by an asterisk. Data noted by an asterisk were derived from data supplied in the original report. Caution should be exercised in interpreting the values of "transverse reinforcement ratio." Some researchers computed the transverse reinforcement ratio as a volumetric ratio, while others computed it in the same manner as the shear reinforcement ratio is computed for a flexural member. Therefore, to insure a uniform interpretation of the term "transverse reinforcement ratio", when using the data given in this report the ratio should be recalculated for the specific purpose at hand, based on the geometric data presented in the sketch of each cross section.

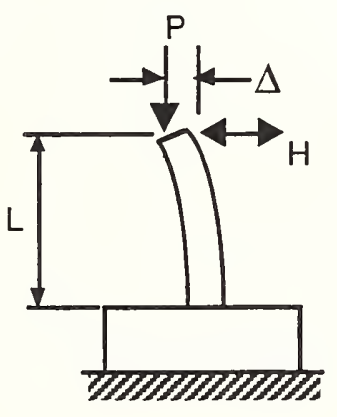

Figure 1: Schematic of Cantilever Specimen

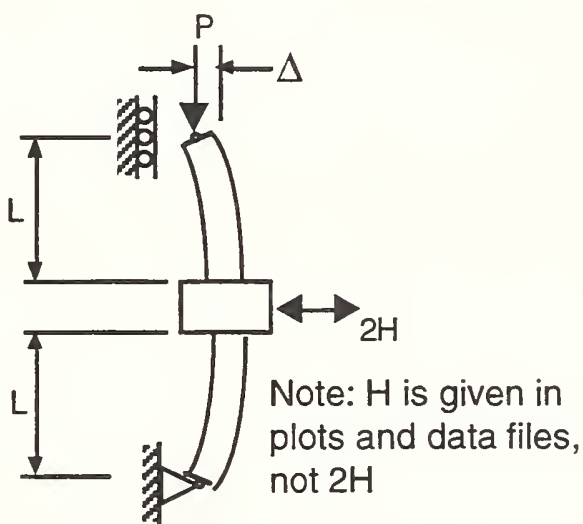

Figure 2: Schematic of Double Ended Specimen

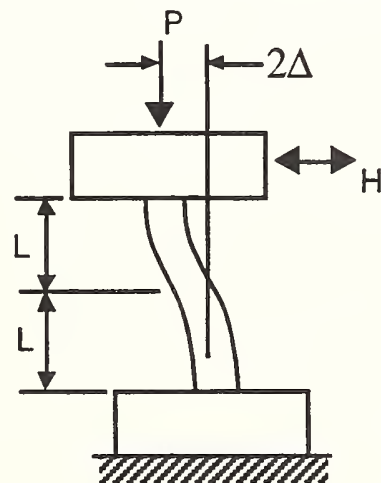

Note: $\Delta$ is given in plots and data files, not $2 \Delta$

Figure 3: Schematic of Double Curvature Specimen 


\section{A: Tests Conducted in New Zealand}

1979 Gill

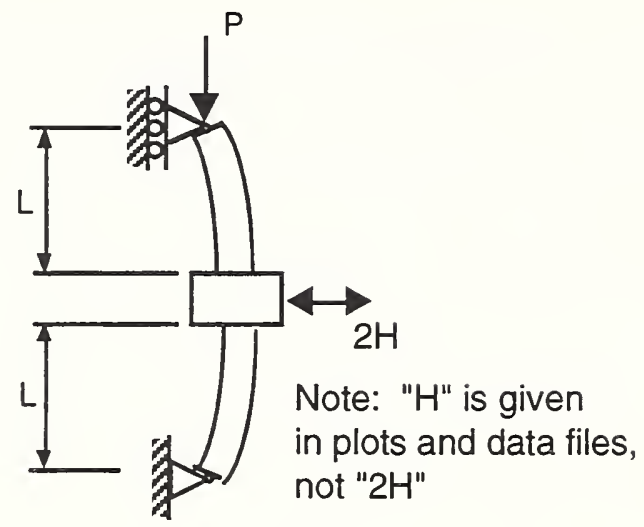

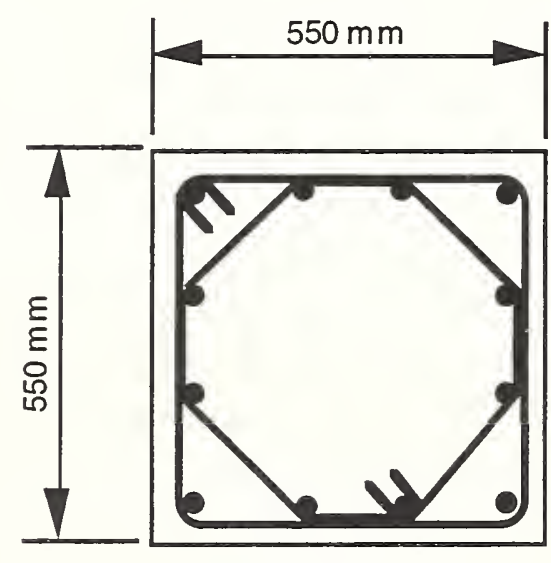

Units 1 and 2

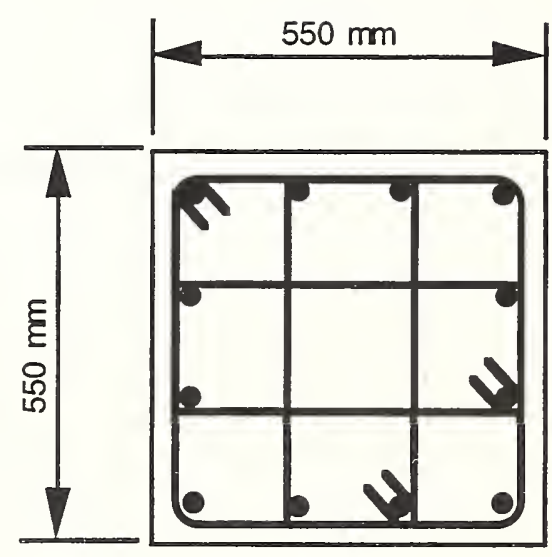

Units 3 and 4

Gill, Wayne Douglas; Park, R.; and Priestley, M.J.N., "Ductility of Rectangular Reinforced Concrete Columns With Axial Load," Report 79-1, Department of Civil Engineering, University of Canterbury, Christchurch, New Zealand, February 1979, 136 pages.

Also reported in Park, R., Priestley, M.J.N., and Gill, W.D., "Ductility of SquareConfined Concrete Columns," Journal of the Structural Division, ASCE, Vol. 108, No. ST4, April 1982, pp. 929-950.

Master of Engineering Report by Gill. Four full-size column sections were subjected to a static cyclic lateral load sequence over a range of axial compressive loads. Two different tie arrangements, both conforming to DZ3101 requirements, were used. These tests were conducted using a double-ended specimen, a self-reacting load frame with a hydraulic ram to apply cyclic lateral load, and a universal testing machine to apply constant axial load. 


\begin{tabular}{|c|c|c|c|c|c|c|}
\hline \multirow{2}{*}{$\begin{array}{c}\text { Unit } \\
\text { No. }\end{array}$} & \multicolumn{3}{|c|}{ In Plastic Hinge Region } & \multicolumn{3}{c|}{ Outside Plastic Hinge Region } \\
\cline { 2 - 7 } & $\begin{array}{c}\text { No. of } \\
\text { Hoop Sets }\end{array}$ & $\begin{array}{c}\text { Bar dia. } \\
\mathrm{mm}\end{array}$ & $\begin{array}{c}\text { Spacing } \\
\text { (ctrs.) mm }\end{array}$ & $\begin{array}{c}\text { No. of } \\
\text { Hoop Sets }\end{array}$ & $\begin{array}{c}\text { Bar dia. } \\
\mathrm{mm}\end{array}$ & $\begin{array}{c}\text { Spacing } \\
\text { (ctrs.) mm }\end{array}$ \\
\hline 1 & 8 & 10 & 80 & 5 & 10 & 135 \\
\hline 2 & 8 & 12 & 75 & 3 & 12 & 210 \\
\hline 3 & 8 & 10 & 75 & 6 & 10 & 105 \\
\hline 4 & 10 & 12 & 72 & 3 & 12 & 200 \\
\hline
\end{tabular}

\begin{tabular}{|c|c|c|c|c|c|c|c|}
\hline \multirow{2}{*}{$\begin{array}{l}\text { Unit } \\
\text { No. }\end{array}$} & $\begin{array}{c}\text { Concrete } \\
\text { Strength, } \\
\mathrm{MPa}\end{array}$ & $\begin{array}{c}\text { Axial } \\
\text { Load } \\
\mathrm{kN}\end{array}$ & \multirow{2}{*}{$\begin{array}{c}\text { Axial Load } \\
\end{array}$} & & \multicolumn{2}{|c|}{$\begin{array}{c}\text { Longitudinal } \\
\text { Reinforcement }\end{array}$} & \multicolumn{2}{|c|}{$\begin{array}{c}\text { Transverse } \\
\text { Reinforcement }\end{array}$} \\
\cline { 5 - 8 } & & & $\rho$ & $\begin{array}{c}\mathrm{f}_{\mathrm{y}} \\
\mathrm{MPa}\end{array}$ & $\rho$ & $\begin{array}{c}\mathrm{f}_{\mathrm{y}} \\
\mathrm{MPa}\end{array}$ \\
\hline 1 & 23.1 & 1815 & 0.260 & 0.0179 & 375 & 0.015 & 297 \\
\hline 2 & 41.4 & 2680 & 0.214 & 0.0179 & 375 & 0.023 & 316 \\
\hline 3 & 21.4 & 2719 & 0.420 & 0.0179 & 375 & 0.020 & 297 \\
\hline 4 & 23.5 & 4265 & 0.600 & 0.0179 & 375 & 0.035 & 294 \\
\hline
\end{tabular}

Longitudinal steel - Grade 380 deformed bars, twelve $24 \mathrm{~mm}$ dia. with $50 \mathrm{~mm}$ cover. Hoop steel - Grade 275 plain round bars.

Half length of specimen, $\mathrm{L}=1.2 \mathrm{~m}$

Data Files:

$$
\begin{aligned}
& \text { GILL79S1.WK1 } \\
& \text { GILL79S2.WK1 } \\
& \text { GILL79S3.WK1 } \\
& \text { GILL79S4.WK1 }
\end{aligned}
$$




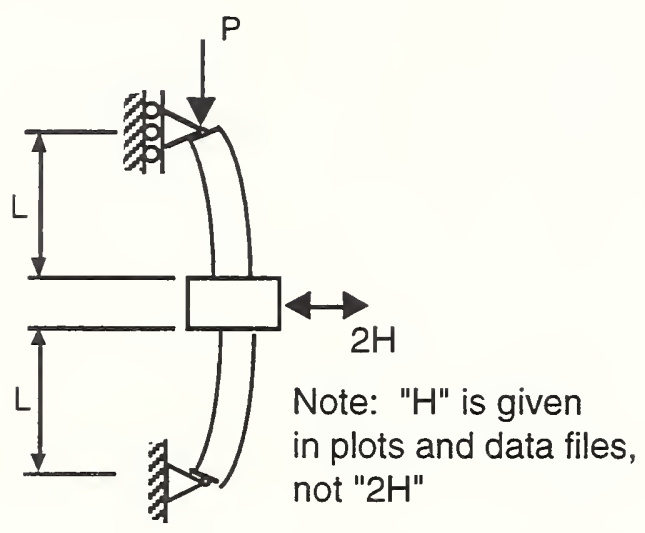

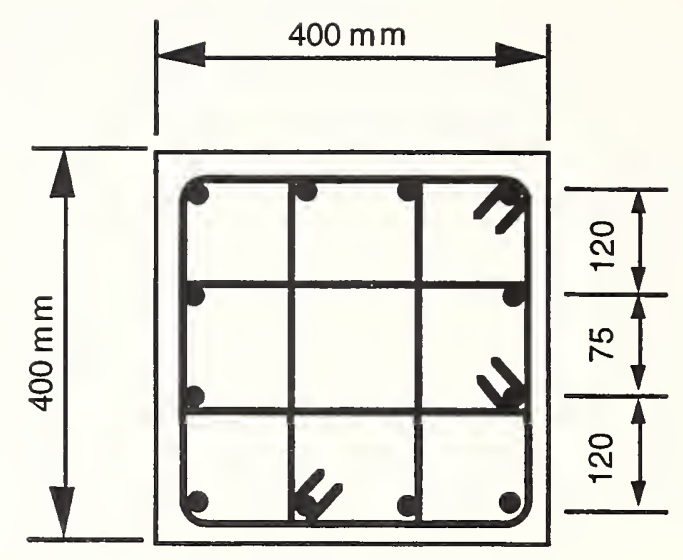

Specimens 3 and 4

Ang Beng Ghee; Priestley, M.J.N.; and Park, R., "Ductility of Reinforced Concrete Bridge Piers Under Seismic Loading," Report 81-3, Department of Civil Engineering, University of Canterbury, Christchurch, New Zealand, February 1981, 109 pages.

Ang tested four columns designed according to the Second Draft (1980) of the Concrete Design Code DZ3101 for different load levels. Only two involved square (rectangular) columns. These tests were conducted using a double-ended specimen, a self-reacting load frame with a hydraulic ram to apply cyclic lateral load, and a universal testing machine to apply constant axial load.

\begin{tabular}{|c|c|c|c|c|c|c|}
\hline Unit & \multicolumn{3}{|c|}{ In Plastic Hinge Region } & \multicolumn{3}{c|}{ Outside Plastic Hinge Region } \\
\cline { 2 - 7 } & $\begin{array}{c}\text { No. of } \\
\text { Hoop Sets }\end{array}$ & $\begin{array}{c}\text { Bar dia. } \\
\text { mm }\end{array}$ & $\begin{array}{c}\text { Spacing } \\
\text { (ctrs.) mm }\end{array}$ & $\begin{array}{c}\text { No. of } \\
\text { Hoop Sets }\end{array}$ & $\begin{array}{c}\text { Bar dia. } \\
\text { mm }\end{array}$ & $\begin{array}{c}\text { Spacing } \\
\text { (ctrs.) mm }\end{array}$ \\
\hline 3 & 5 & 12 & 100 & 6 & 10 & 180 \\
\hline 4 & 5 & 10 & 90 & 6 & 10 & 180 \\
\hline
\end{tabular}

\begin{tabular}{|c|c|c|c|c|c|c|c|}
\hline $\begin{array}{c}\text { Unit } \\
\text { No. }\end{array}$ & $\begin{array}{c}\text { Concrete } \\
\text { Strength, } \\
\mathrm{MPa}\end{array}$ & $\begin{array}{c}\text { Axial } \\
\text { Load } \\
\mathrm{kN}\end{array}$ & \multirow{2}{*}{$\begin{array}{c}\text { Axial Load } \\
\text { Axial Capacity }\end{array}$} & \multicolumn{2}{|c|}{$\begin{array}{c}\text { Longitudinal } \\
\text { Reinforcement }\end{array}$} & \multicolumn{2}{|c|}{$\begin{array}{c}\text { Transverse } \\
\text { Reinforcement }\end{array}$} \\
\cline { 5 - 8 } & & & & $\rho$ & $\begin{array}{c}\mathrm{f}_{\mathrm{y}} \\
\mathrm{MPa}\end{array}$ & $\rho$ & $\begin{array}{c}\mathrm{f}_{\mathrm{y}} \\
\mathrm{MPa}\end{array}$ \\
\hline 3 & 23.6 & 1435 & 0.38 & 0.0151 & 427 & 0.02832 & 320 \\
\hline 4 & 25.0 & 840 & 0.21 & 0.0151 & 427 & 0.02218 & 280 \\
\hline
\end{tabular}

Longitudinal steel - Grade 380 deformed bars, twelve $16 \mathrm{~mm}$ dia. with $34.5 \mathrm{~mm}$ cover. Hoop steel - Grade 275 plain round bars.

Half length of specimen, $\mathrm{L}=1.6 \mathrm{~m}$

Data Files:

ANG81U3.WK1

ANG81U4.WK1 

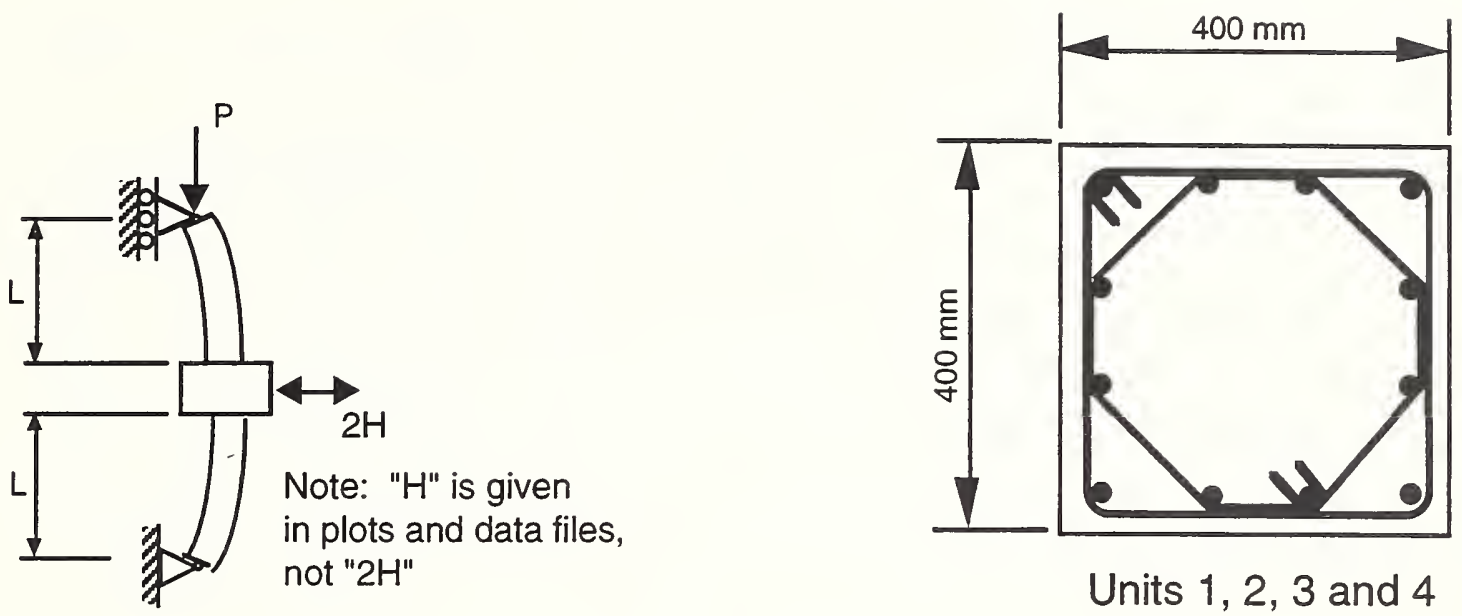

Units 1, 2, 3 and 4

Soesianawati, M.T.; Park, R.; and Priestley, M.J.N., "Limited Ductility Design of Reinforced Concrete Columns," Report 86-10, Department of Civil Engineering, University of Canterbury, Christchurch, New Zealand, March 1986, 208 pages.

Four columns of square cross-section were tested; the quantity of transverse confining steel used ranged from 17 to 46 percent of the NZS 3101:1982 recommended quantity for ductile detailing. These tests were conducted using a double-ended specimen, a self-reacting load frame with a hydraulic ram to apply cyclic lateral load, and a universal testing machine to apply constant axial load.

\begin{tabular}{|c|c|c|c|c|c|c|}
\hline \multirow{2}{*}{$\begin{array}{c}\text { Unit } \\
\text { No. }\end{array}$} & \multicolumn{3}{|c|}{ In Plastic Hinge Region } & \multicolumn{3}{c|}{ Outside Plastic Hinge Region } \\
\cline { 2 - 7 } & $\begin{array}{c}\text { No. of } \\
\text { Hoop Sets }\end{array}$ & $\begin{array}{c}\text { Bar dia. } \\
\text { mm }\end{array}$ & $\begin{array}{c}\text { Spacing } \\
\text { (ctrs.) mm }\end{array}$ & $\begin{array}{c}\text { No. of } \\
\text { Hoop Sets }\end{array}$ & $\begin{array}{c}\text { Bar dia. } \\
\text { mm }\end{array}$ & $\begin{array}{c}\text { Spacing } \\
\text { (ctrs.) mm }\end{array}$ \\
\hline 1 & 5 & 7 & 85 & 6 & 7 & 170 \\
\hline 2 & 8 & 8 & 78 & 5 & 8 & 156 \\
\hline 3 & 7 & 7 & 91 & 5 & 7 & 182 \\
\hline 4 & 7 & 6 & 94 & 5 & 6 & 186 \\
\hline
\end{tabular}

\begin{tabular}{|c|c|c|c|c|c|c|c|}
\hline $\begin{array}{c}\text { Unit } \\
\text { No. }\end{array}$ & $\begin{array}{c}\text { Concrete } \\
\text { Strength, } \\
\mathrm{MPa}\end{array}$ & $\begin{array}{c}\text { Axial } \\
\text { Load } \\
\mathrm{kN}\end{array}$ & \multirow{2}{*}{$\begin{array}{c}\text { Axial Load } \\
\text { Axial Capacity }\end{array}$} & \multicolumn{2}{|c|}{$\begin{array}{c}\text { Longitudinal } \\
\text { Reinforcement }\end{array}$} & \multicolumn{2}{|c|}{$\begin{array}{c}\text { Transverse } \\
\text { Reinforcement }\end{array}$} \\
\cline { 5 - 8 } & & & $\rho$ & $\begin{array}{c}\mathrm{f}_{\mathrm{y}} \\
\mathrm{MPa}\end{array}$ & $\rho$ & $\begin{array}{c}\mathrm{f}_{\mathrm{y}} \\
\mathrm{MPa}\end{array}$ \\
\hline 1 & 46.5 & 744 & 0.1 & 0.0151 & 446 & 0.0086 & 364 \\
\hline 2 & 44.0 & 2112 & 0.3 & 0.0151 & 446 & 0.0122 & 360 \\
\hline 3 & 44.0 & 2112 & 0.3 & 0.0151 & 446 & 0.0080 & 364 \\
\hline 4 & 40.0 & 1920 & 0.3 & 0.0151 & 446 & 0.0057 & 255 \\
\hline
\end{tabular}

Longitudinal steel - Grade 380 deformed bars, twelve $16 \mathrm{~mm}$ dia. bars

Hoop steel - Grade 275 plain round bars. Clear cover to transverse bars $=13 \mathrm{~mm}$.

Half length of specimen, $L=1.6 \mathrm{~m}$

Data Files:

SOES86U1.WK1

SOES86U2.WK1

SOES86U3.WK1

SOES86U4.WK1 

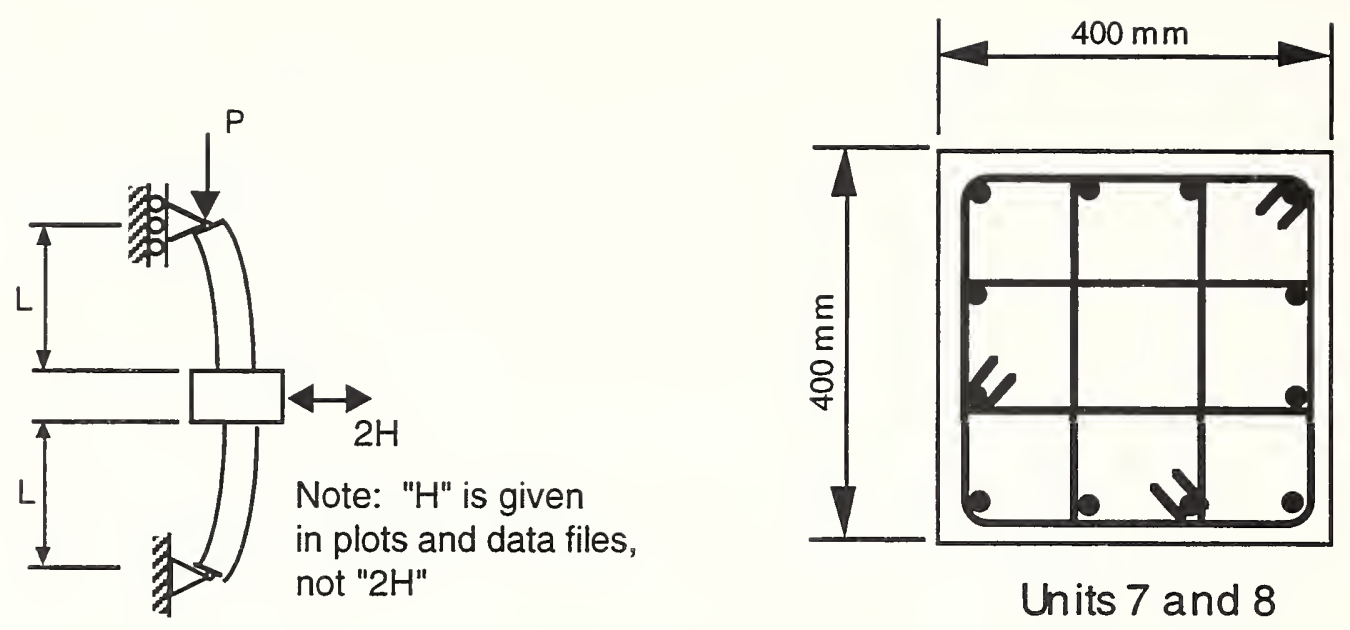

Zahn, F.A.; Park, R.; and Priestley, M.J.N., "Design of Reinforced Concrete Bridge Columns for Strength and Ductility," Report 86-7, Department of Civil Engineering, University of Canterbury, Christchurch, New Zealand, March 1986, 330 pages.

Originally written as a $\mathrm{PhD}$ thesis by Zahn. Sixteen specimens were tested with various shapes and load orientation, but only Units 7 and 8 were square with loading along a principal axis of the cross-section. These tests were conducted using a double-ended specimen, a self-reacting load frame with a hydraulic ram to apply cyclic lateral load, and a universal testing machine to apply constant axial load.

\begin{tabular}{|c|c|c|c|}
\hline \multirow{2}{*}{$\begin{array}{c}\text { Unit } \\
\text { No. }\end{array}$} & \multicolumn{3}{|c|}{ In Plastic Hinge Region } \\
\cline { 2 - 4 } & $\begin{array}{c}\text { No. of } \\
\text { Hoop Sets }\end{array}$ & $\begin{array}{c}\text { Bar dia. } \\
\text { mm }\end{array}$ & $\begin{array}{c}\text { Spacing } \\
\text { (ctrs.) mm }\end{array}$ \\
\hline 7 & 4 & 10 & 117 \\
\hline 8 & 7 & 10 & 92 \\
\hline
\end{tabular}

\begin{tabular}{|c|c|c|c|c|c|c|c|}
\hline \multirow{2}{*}{$\begin{array}{c}\text { Unit } \\
\text { No. }\end{array}$} & $\begin{array}{c}\text { Concrete } \\
\text { Strength, } \\
\mathrm{MPa}\end{array}$ & $\begin{array}{c}\text { Axial } \\
\text { Load } \\
\mathrm{kN}\end{array}$ & \multirow{2}{*}{$\begin{array}{c}\text { Axial Load } \\
\text { Axial Capacity }\end{array}$} & \multicolumn{2}{|c|}{$\begin{array}{c}\text { Longitudinal } \\
\text { Reinforcement }\end{array}$} & \multicolumn{2}{|c|}{$\begin{array}{c}\text { Transverse } \\
\text { Reinforcement }\end{array}$} \\
\cline { 5 - 8 } & & & & $\rho$ & $\begin{array}{c}\mathrm{f}_{\mathrm{y}} \\
\mathrm{MPa}\end{array}$ & $\rho$ & $\begin{array}{c}\mathrm{f}_{\mathrm{y}} \\
\mathrm{MPa}\end{array}$ \\
\hline 7 & 28.3 & 1010 & 0.23 & 0.0151 & 440 & 0.0156 & 466 \\
\hline 8 & 40.1 & 2502 & 0.39 & 0.0151 & 440 & 0.0199 & 466 \\
\hline
\end{tabular}

Longitudinal steel - Grade 380 deformed bars, twelve $16 \mathrm{~mm}$ dia. bars

Hoop steel - Grade 380 plain round bars. Clear cover to transverse bars $=13 \mathrm{~mm}$.

Half length of specimen, $\mathrm{L}=1.6 \mathrm{~m}$

Data Files:

ZAHN86U7.WK1

ZAHN86U8.WK1 

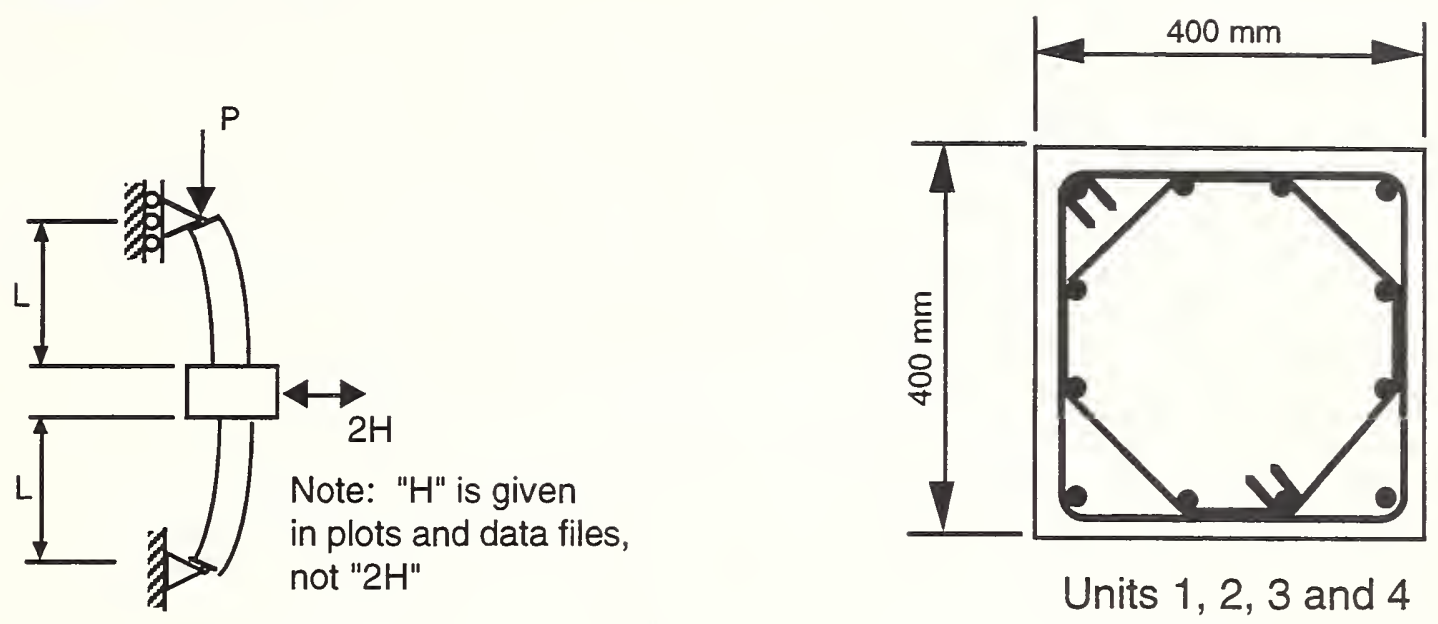

Watson, Soesianawati; and Park, R., "Design of Reinforced Concrete Frames of Limited Ductility," Report 89-4, Department of Civil Engineering, University of Canterbury, Christchurch, New Zealand, January 1989, 232 pages.

$\mathrm{PhD}$ thesis by Watson (née Soesianawati). Seven specimens were tested, but only Units 5 through 9 were rectangular. These tests were conducted using a double-ended specimen, a selfreacting load frame with a hydraulic ram to apply cyclic lateral load, and a universal testing machine to apply constant axial load.

\begin{tabular}{|c|c|c|c|}
\hline \multirow{2}{*}{$\begin{array}{c}\text { Unit } \\
\text { No. }\end{array}$} & \multicolumn{3}{|c|}{ In Plastic Hinge Region } \\
\cline { 2 - 4 } & $\begin{array}{c}\text { No. of } \\
\text { Hoop Sets }\end{array}$ & $\begin{array}{c}\text { Bar dia. } \\
\text { mm }\end{array}$ & $\begin{array}{c}\text { Spacing } \\
\text { (ctrs.) mm }\end{array}$ \\
\hline 5 & 8 & 8 & 81 \\
\hline 6 & 7 & 6 & 96 \\
\hline 7 & 7 & 12 & 96 \\
\hline 8 & 8 & 8 & 77 \\
\hline 9 & 12 & 12 & 52 \\
\hline
\end{tabular}

\begin{tabular}{|c|c|c|c|c|c|c|c|}
\hline \multirow{2}{*}{$\begin{array}{c}\text { Unit } \\
\text { No. }\end{array}$} & $\begin{array}{c}\text { Concrete } \\
\text { Strength, } \\
\mathrm{MPa}\end{array}$ & $\begin{array}{c}\text { Axial } \\
\text { Load } \\
\mathrm{kN}\end{array}$ & \multirow{2}{*}{$\begin{array}{c}\text { Axial Load } \\
\text { Axial Capacity }\end{array}$} & \multicolumn{2}{|c|}{$\begin{array}{c}\text { Longitudinal } \\
\text { Reinforcement }\end{array}$} & \multicolumn{2}{|c|}{$\begin{array}{c}\text { Transverse } \\
\text { Reinforcement }\end{array}$} \\
\cline { 5 - 8 } & & & & $\rho$ & $\begin{array}{c}\mathrm{f}_{\mathrm{y}} \\
\mathrm{MPa}\end{array}$ & $\rho^{*}$ & $\begin{array}{c}\mathrm{f}_{\mathrm{y}} \\
\mathrm{MPa}\end{array}$ \\
\hline 5 & 41 & 3280 & 0.5 & 0.0151 & 474 & 0.0066 & 372 \\
\hline 6 & 40 & 3200 & 0.5 & 0.0151 & 474 & 0.0032 & 388 \\
\hline 7 & 42 & 4704 & 0.7 & 0.0151 & 474 & 0.0126 & 308 \\
\hline 8 & 39 & 4368 & 0.7 & 0.0151 & 474 & 0.0070 & 372 \\
\hline 9 & 40 & 4480 & 0.7 & 0.0151 & 474 & 0.0233 & 308 \\
\hline
\end{tabular}

Longitudinal steel - Grade 380 deformed bars, twelve $16 \mathrm{~mm}$ dia. bars

Hoop steel - Grade 275 plain round bars. Clear cover to transverse bars $=13 \mathrm{~mm}$.

* Calculated from data given in report

Half length of specimen, $\mathrm{L}=1.6 \mathrm{~m}$

Data Files:

WAT89U5.WK1

WAT89U6.WK1

WAT89U7.WK1

WAT89U8.WK1

WAT89U9.WK1 


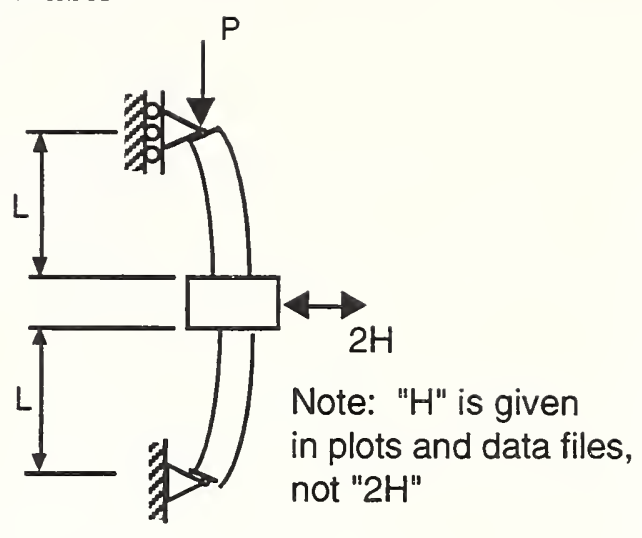

Specimens 1 to 4

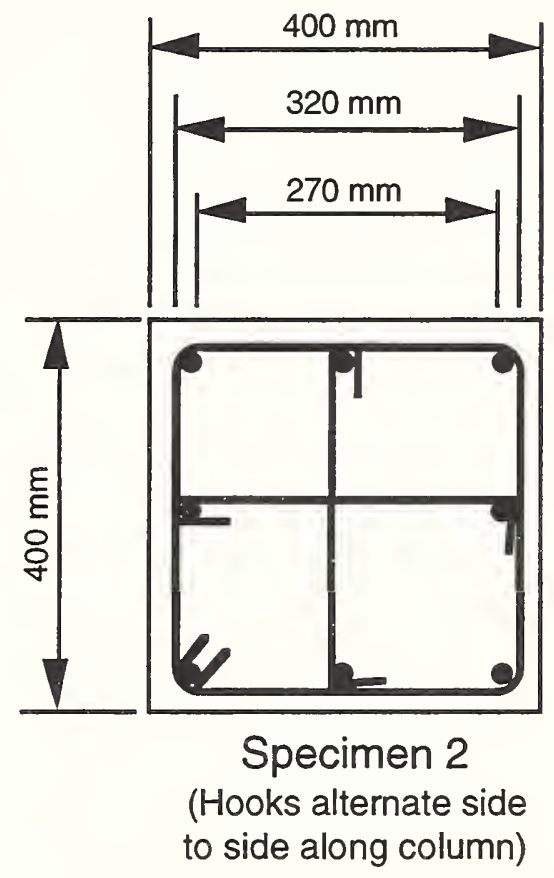

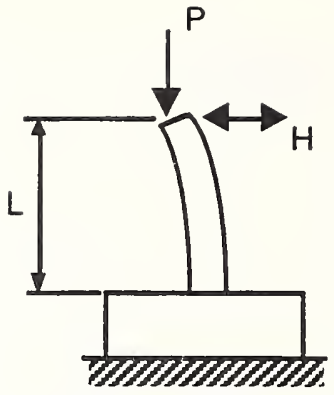

Specimens 5 to 8

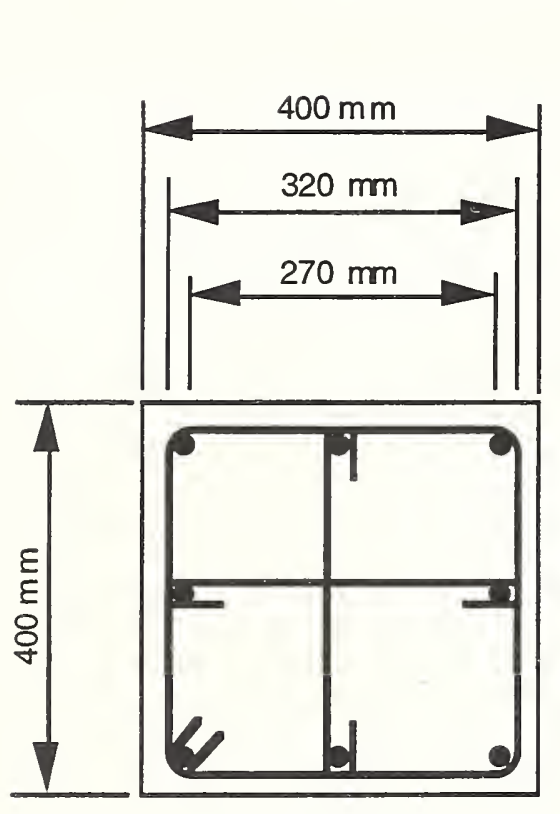

Specimen 1

a

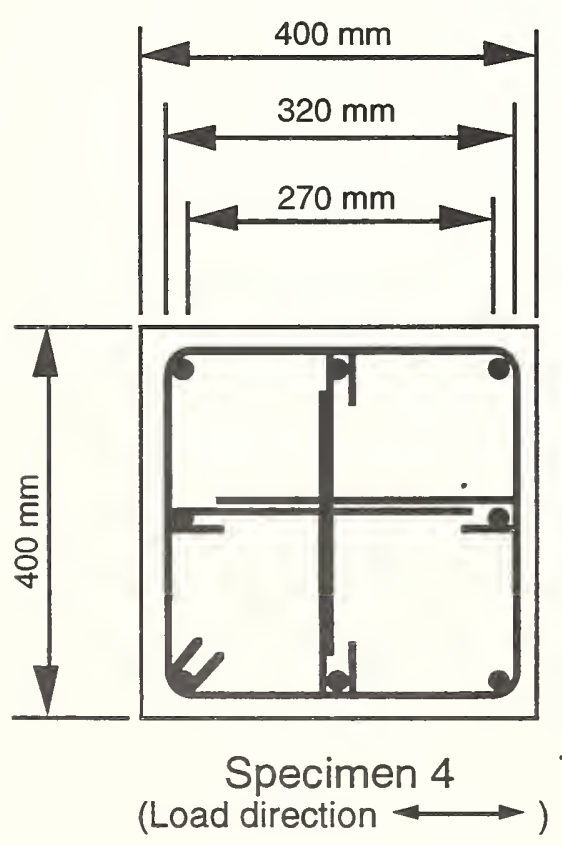

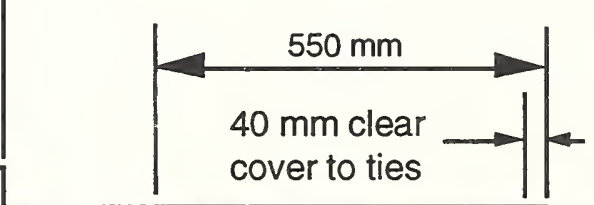

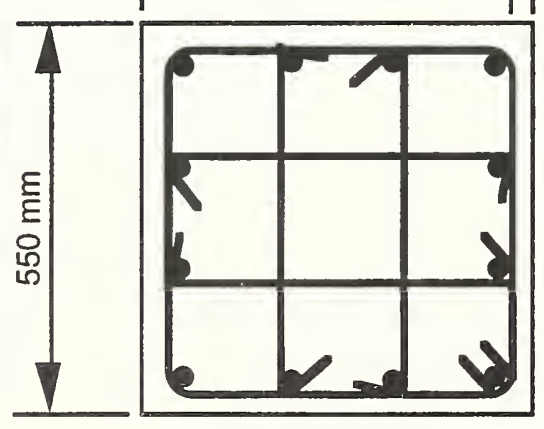

Specimens 5 and 7
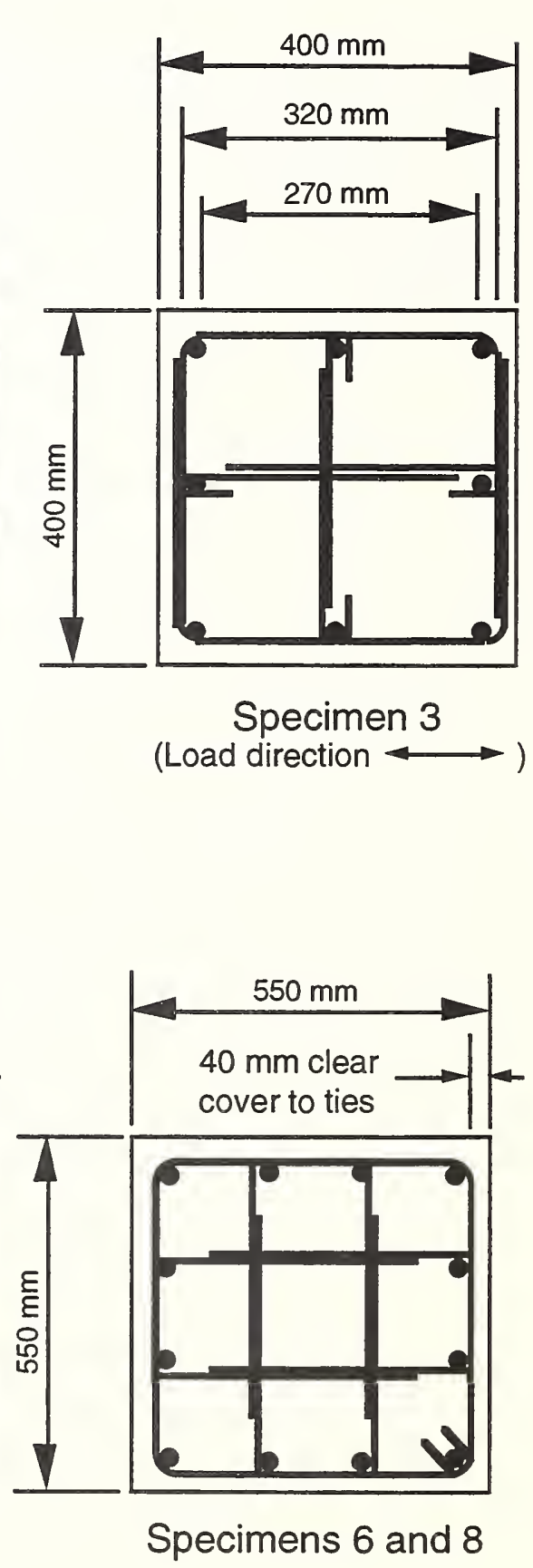
Tanaka, H.; and Park, R., "Effect of Lateral Confining Reinforcement on the Ductile Behaviour of Reinforced Concrete Columns," Report 90-2, Department of Civil Engineering, University of Canterbury, June 1990, 458 pages.

$\mathrm{PhD}$ thesis by Tanaka. Eight columns with square cross sections were tested. (One specimen with a rectangular cross section was also tested, as described in the next section). Specimens one through four were conducted using a double-ended specimen, a self-reacting load frame with a hydraulic ram to apply cyclic lateral load, and a universal testing machine to apply constant axial load. Specimens five through eight incorporated a true cantilever column setup.

\begin{tabular}{|c|c|c|c|}
\hline \multirow{2}{*}{$\begin{array}{c}\text { Unit } \\
\text { No. }\end{array}$} & \multicolumn{3}{|c|}{ In Plastic Hinge Region } \\
\cline { 2 - 4 } & $\begin{array}{c}\text { No. of } \\
\text { Hoop Sets }\end{array}$ & $\begin{array}{c}\text { Bar dia. } \\
\text { mm }\end{array}$ & $\begin{array}{c}\text { Spacing } \\
\text { (ctrs.) mm }\end{array}$ \\
\hline 1 & 6 & 12 & 80 \\
\hline 2 & 6 & 12 & 80 \\
\hline 3 & 6 & 12 & 80 \\
\hline 4 & 6 & 12 & 80 \\
\hline 5 & 6 & 12 & 110 \\
\hline 6 & 6 & 12 & 110 \\
\hline 7 & 7 & 12 & 90 \\
\hline 8 & 7 & 12 & 90 \\
\hline
\end{tabular}

\begin{tabular}{|c|c|c|c|c|c|c|c|}
\hline \multirow{2}{*}{$\begin{array}{l}\text { Unit } \\
\text { No. }\end{array}$} & $\begin{array}{c}\text { Concrete } \\
\text { Strength, } \\
\mathrm{MPa}\end{array}$ & $\begin{array}{c}\text { Axial } \\
\text { Load } \\
\mathrm{kN}^{*}\end{array}$ & $\mathrm{~A}$ & $\begin{array}{c}\text { Axial Load } \\
\text { Axial Capacity }\end{array}$ & \multicolumn{2}{|c|}{$\begin{array}{c}\text { Longitudinal } \\
\text { Reinforcement }\end{array}$} & \multicolumn{2}{|c|}{$\begin{array}{c}\text { Transverse } \\
\text { Reinforcement }\end{array}$} \\
\cline { 5 - 8 } & & & $\rho$ & $\begin{array}{c}\mathrm{f}_{\mathrm{y}} \\
\mathrm{MPa}\end{array}$ & $\rho$ & $\begin{array}{c}\mathrm{f}_{\mathrm{y}} \\
\mathrm{MPa}_{\mathrm{y}}\end{array}$ \\
\hline 1 & 25.6 & 819 & 0.2 & 0.0157 & 474 & 0.0255 & 333 \\
\hline 2 & 25.6 & 819 & 0.2 & 0.0157 & 474 & 0.0255 & 333 \\
\hline 3 & 25.6 & 819 & 0.2 & 0.0157 & 474 & 0.0255 & 333 \\
\hline 4 & 25.6 & 819 & 0.2 & 0.0157 & 474 & 0.0255 & 333 \\
\hline 5 & 32.0 & 968 & 0.1 & 0.0125 & 511 & 0.0170 & 325 \\
\hline 6 & 32.0 & 968 & 0.1 & 0.0125 & 511 & 0.0170 & 325 \\
\hline 7 & 32.1 & 2913 & 0.3 & 0.0125 & 511 & 0.0208 & 325 \\
\hline 8 & 32.1 & 2913 & 0.3 & 0.0125 & 511 & 0.0208 & 325 \\
\hline
\end{tabular}

Longitudinal steel - Grade 380 deformed bars, $20 \mathrm{~mm}$ dia. bars

Hoop steel - Grade 275 deformed bars. Clear cover to transverse bars $=40 \mathrm{~mm}$.

* Calculated from data given in report

Half length of specimens 1 to $4, \mathrm{~L}=1.6 \mathrm{~m}$. Length of specimens 5 to $8, \mathrm{~L}=1.65 \mathrm{~m}$

Data Files:

TANA90U1.WK1

TANA90U2.WK1

TANA90U3.WK1

TANA90U4.WK1

TANA90U5.WK1

TANA90U6.WK1

TANA90U7.WK1

TANA90U8.WK1 

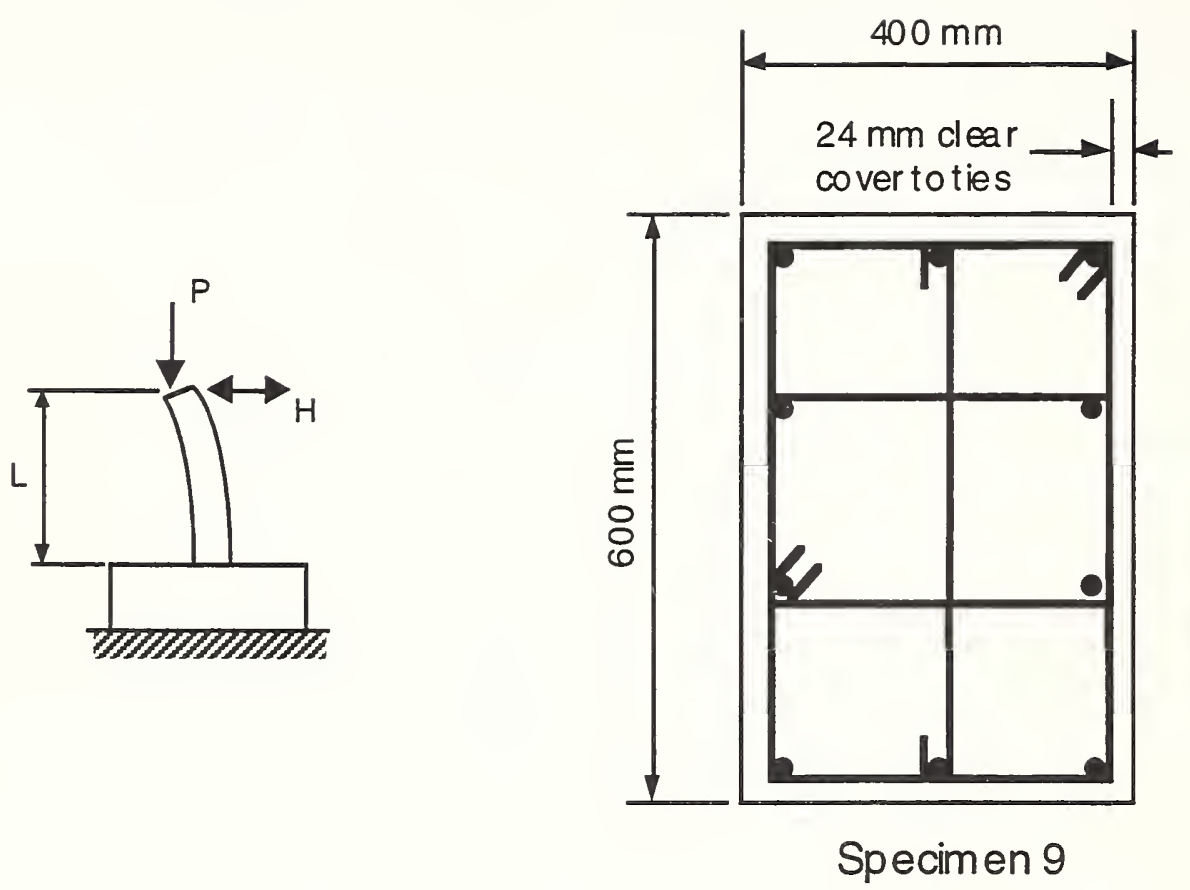

Park, R.; and Paulay, T., "Use of Interlocking Spirals for Transverse Reinforcement in Bridge Columns," Strength and Ductility of Concrete Substructures of Bridges, RRU (Road Research Unit) Bulletin 84, Vol. 1, 1990, pp 77-92.

See also, Tanaka, H.; and Park, R., "Effect of Lateral Confining Reinforcement on the Ductile Behaviour of Reinforced Concrete Columns," Report 90-2, Department of Civil Engineering, University of Canterbury, June 1990, 458 pages, described in the previous section.

One rectangular column with overlapping hoops was tested. The configuration of the specimen was a cantilever, as illustrated above.

\begin{tabular}{|c|c|c|c|}
\hline \multirow{2}{*}{$\begin{array}{c}\text { Unit } \\
\text { No. }\end{array}$} & \multicolumn{3}{|c|}{ In Plastic Hinge Region } \\
\cline { 2 - 4 } & $\begin{array}{c}\text { No. of } \\
\text { Hoop Sets }\end{array}$ & $\begin{array}{c}\text { Bar dia. } \\
\text { mm }\end{array}$ & $\begin{array}{c}\text { Spacing } \\
\text { (ctrs.) mm }\end{array}$ \\
\hline 9 & 9 & 12 & 80 \\
\hline
\end{tabular}

\begin{tabular}{|c|c|c|c|c|c|c|c|}
\hline \multirow{2}{*}{$\begin{array}{c}\text { Unit } \\
\text { No. }\end{array}$} & $\begin{array}{c}\text { Concrete } \\
\text { Strength, } \\
\mathrm{MPa}\end{array}$ & $\begin{array}{c}\text { Axial } \\
\text { Load } \\
\mathrm{kN}\end{array}$ & \multirow{2}{*}{$\begin{array}{c}\text { Axial Load } \\
\text { Axial Capacity }\end{array}$} & \multicolumn{2}{|c|}{$\begin{array}{c}\text { Longitudinal } \\
\text { Reinforcement }\end{array}$} & \multicolumn{2}{|c|}{$\begin{array}{c}\text { Transverse } \\
\text { Reinforcement }\end{array}$} \\
\cline { 5 - 8 } & & & $\rho$ & $\begin{array}{c}\mathrm{f}_{\mathrm{y}} \\
\mathrm{MPa}\end{array}$ & $\rho$ & $\begin{array}{c}\mathrm{f}_{\mathrm{y}} \\
\mathrm{MPa}\end{array}$ \\
\hline 9 & 26.9 & 646 & 0.1 & 0.0188 & 432 & 0.0217 & 305 \\
\hline
\end{tabular}

Longitudinal steel - Grade 380 deformed bars, $24 \mathrm{~mm}$ dia. bars

Hoop steel - Grade 275 plain bars. Clear cover to transverse bars $=24 \mathrm{~mm}$.

* Calculated from data given in report

Length of specimen, $\mathrm{L}=1.784 \mathrm{~m}$

Data File:

TANA90U9.WK1 


\section{B. Tests Conducted in Japan}

\section{Arakawa, Arai, Egashira, and Fujita}
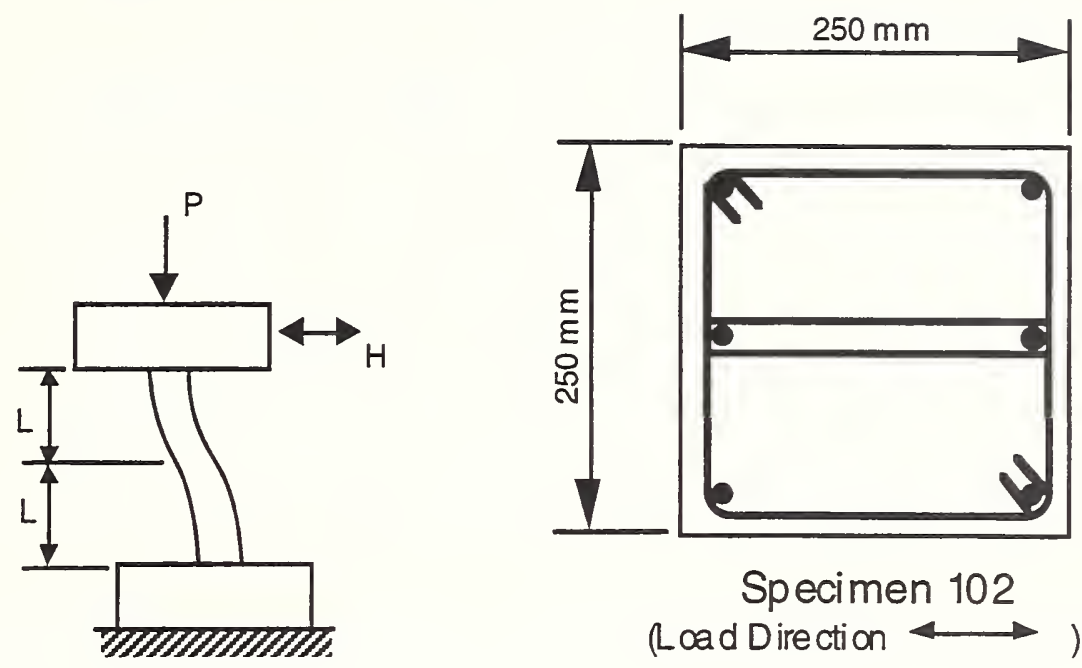

Arakawa, Takashi; Arai, Yasuyuki; Egashira, Keiichi; and Fujita, Yutaka, "Effects of the Rate of Cyclic Loading on the Load-Carrying Capacity and Inelastic Behavior of Reinforced Concrete Columns," Transactions of the Japan Concrete Institute, Vol. 4, 1982, pp 485-492.

Ten double-curvature specimens were tested under constant axial load and cyclic lateral load. The main variable was the rate of lateral loading. A digitizable analog plot was available from the authors for only one of the tests, specimen 102. This specimen was tested at a cyclic lateral load rate of $0.5 \mathrm{~Hz}$.

\begin{tabular}{|c|c|c|}
\hline \multirow{2}{*}{$\begin{array}{c}\text { Unit } \\
\text { No. }\end{array}$} & $\begin{array}{c}\text { Lateral Reinforcement } \\
\text { mm }\end{array}$ & $\begin{array}{c}\text { Spacing } \\
\text { (ctrs.) } \mathrm{mm}\end{array}$ \\
\hline 102 & 5.5 & 32 \\
\hline
\end{tabular}

\begin{tabular}{|c|c|c|c|c|c|c|c|}
\hline $\begin{array}{l}\text { Unit } \\
\text { No. }\end{array}$ & $\begin{array}{c}\text { Concrete } \\
\text { Strength, } \\
\mathrm{MPa}\end{array}$ & $\begin{array}{c}\text { Axial } \\
\text { Load } \\
\mathrm{kN}^{*}\end{array}$ & Axial Capacity & \multicolumn{2}{|c|}{$\begin{array}{c}\text { Longitudinal } \\
\text { Reinforcement }\end{array}$} & \multicolumn{2}{|c|}{$\begin{array}{c}\text { Transverse } \\
\text { Reinforcement }\end{array}$} \\
\cline { 4 - 8 } & & & $\rho$ & $\begin{array}{c}\mathrm{f}_{\mathrm{y}} \\
\mathrm{MPa}\end{array}$ & $\rho$ & $\begin{array}{c}\mathrm{f}_{\mathrm{y}} \\
\mathrm{MPa}\end{array}$ \\
\hline 102 & 20.6 & 429 & 0.33 & 0.0068 & 392.8 & 0.0118 & 323 \\
\hline
\end{tabular}

Longitudinal steel - six deformed bars, $10 \mathrm{~mm}$ dia.

* Calculated from data given in report

Half height of specimen, $\mathrm{L}=0.375 \mathrm{~m}$

Data Files:

AR82102.WK1 

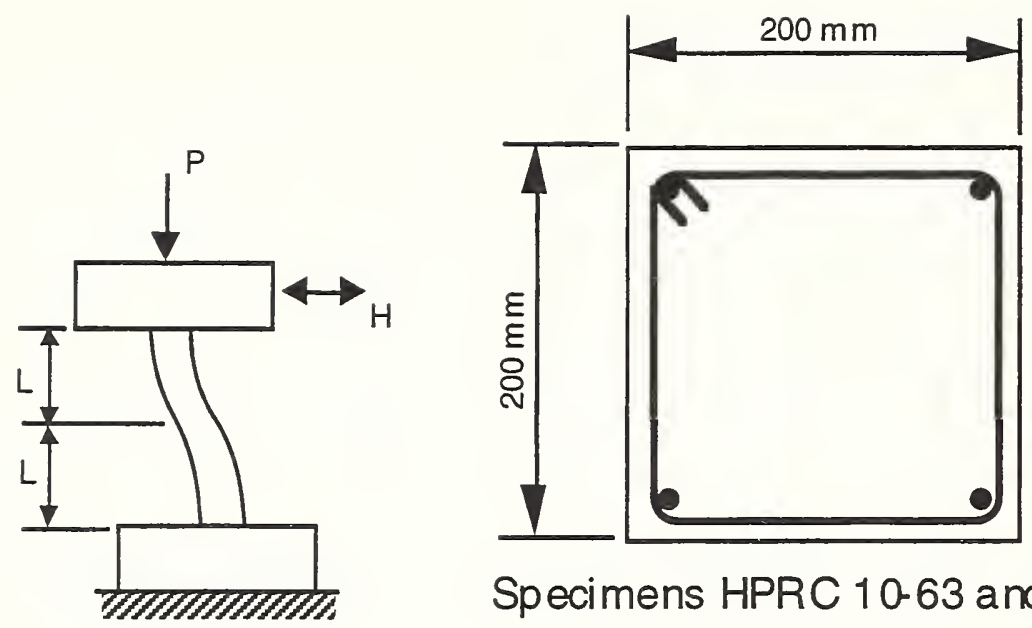

Specimens HPRC 10-63 and 19-32

Nagasaka, Tomoya, "Effectiveness of Steel Fiber as Web Reinforcement in Reinforced Concrete Columns," Transactions of the Japan Concrete Institute, Vol. 4, 1982, pp. 493-500.

Twenty-two double-curvature specimens were tested under constant axial load and cyclic lateral load. The main variable was the use of conventional hoop reinforcement vs. the use of fiber reinforcement. Digitizable analog plots were available from the paper for only two of the tests that had conventional hoop reinforcement and no fiber reinforcement: specimen HPRC 10-63 and HPRC 19-32.

\begin{tabular}{|c|c|c|}
\hline \multirow{2}{*}{$\begin{array}{l}\text { Unit } \\
\text { No. }\end{array}$} & \multicolumn{2}{|c|}{ Lateral Reinforcement } \\
\hline & $\begin{array}{l}\text { Bar dia. } \\
\text { mm }\end{array}$ & $\begin{array}{l}\text { Spacing } \\
\text { (ctrs.) } \mathrm{mm}\end{array}$ \\
\hline HPRC $10-63$ & 6 & 35 \\
\hline HPRC 19-32 & 6 & 20 \\
\hline
\end{tabular}

\begin{tabular}{|c|c|c|c|c|c|c|c|}
\hline $\begin{array}{c}\text { Unit } \\
\text { No. }\end{array}$ & $\begin{array}{c}\text { Concrete } \\
\text { Strength, } \\
\mathrm{MPa}\end{array}$ & $\begin{array}{c}\text { Axial } \\
\mathrm{kNN}\end{array}$ & \multicolumn{2}{|c|}{$\begin{array}{c}\text { Axial Load } \\
\text { Axial Capacity* }\end{array}$} & \multicolumn{2}{|c|}{$\begin{array}{c}\text { Longitudinal } \\
\text { Reinforcement }\end{array}$} & \multicolumn{2}{|c|}{$\begin{array}{c}\text { Transverse } \\
\text { Reinforcement }\end{array}$} \\
\cline { 5 - 8 } & & & & $\rho$ & $\begin{array}{c}\mathrm{f}_{\mathrm{y}} \\
\mathrm{MPa}\end{array}$ & $\rho$ & $\begin{array}{c}\mathrm{f}_{\mathrm{y}} \\
\mathrm{MPa}\end{array}$ \\
\hline $\begin{array}{c}\text { HPRC } \\
10-63\end{array}$ & 21.6 & 147 & 0.17 & 0.0133 & 371 & 0.0081 & 344 \\
\hline $\begin{array}{c}\text { HPRC } \\
19-32\end{array}$ & 21.0 & 294 & 0.34 & 0.0133 & 371 & 0.0139 & 344 \\
\hline
\end{tabular}

Longitudinal steel - four deformed bars, $13 \mathrm{~mm}$ dia.

*Calculated from data given in report

Half height of specimen, $\mathrm{L}=0.30 \mathrm{~m}$

Data Files:

NAG1063.WK1

NAG1932.WK1 

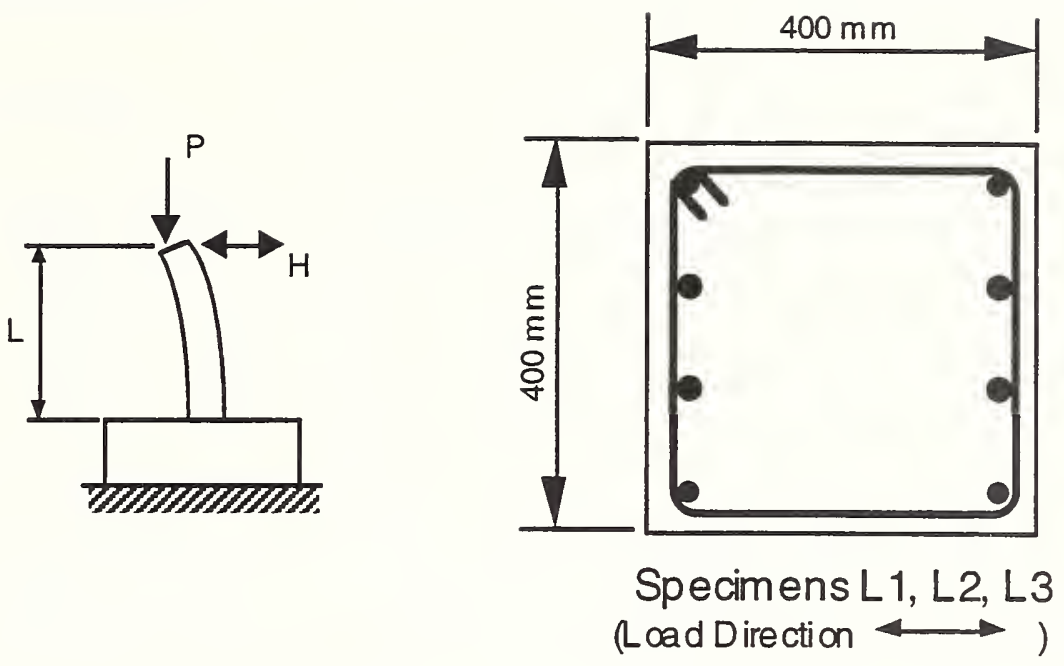

Ohno, Tomonori; and Nishioka, Takashi, "An Experimental Study on Energy Absorption Capacity of Columns in Reinforced Concrete Structures," Proceedings of the JSCE, Structural Engineering/Earthquake Engineering, Vol. 1, No 2., October 1984, pp. 137-147.

Five cantilevered columns were tested under constant axial load and cyclic lateral load. The variables studied were the lateral load pattern and the level of axial load. Digitizable analog plots were available for only three of the tests: specimens L1, L2 and L3. The lateral load pattern for specimen L1 was very severe, and only two complete cycles were applied.

\begin{tabular}{|c|c|c|}
\hline \multirow{2}{*}{$\begin{array}{l}\text { Unit } \\
\text { No. }\end{array}$} & \multicolumn{2}{|c|}{ Lateral Reinforcement } \\
\cline { 2 - 3 } & $\begin{array}{c}\text { Bar dia. } \\
\text { mm }\end{array}$ & $\begin{array}{c}\text { Spacing } \\
\text { (ctrs.) } \mathrm{mm}\end{array}$ \\
\hline L1 & 9 & 100 \\
\hline L2 & 9 & 100 \\
\hline L3 & 9 & 100 \\
\hline
\end{tabular}

\begin{tabular}{|c|c|c|c|c|c|c|c|}
\hline $\begin{array}{l}\text { Unit } \\
\text { No. }\end{array}$ & $\begin{array}{c}\text { Concrete } \\
\text { Strength, } \\
\mathrm{MPa}\end{array}$ & $\begin{array}{c}\text { Axial } \\
\text { Load } \\
\mathrm{kN*}\end{array}$ & \multirow{2}{*}{$\begin{array}{c}\text { Axial Load } \\
\text { Axial Capacity* }\end{array}$} & \multicolumn{2}{|c|}{$\begin{array}{c}\text { Longitudinal } \\
\text { Reinforcement }\end{array}$} & \multicolumn{2}{|c|}{$\begin{array}{c}\text { Transverse } \\
\text { Reinforcement }\end{array}$} \\
\cline { 5 - 8 } & & & $\rho *$ & $\begin{array}{c}\mathrm{f}_{\mathrm{y}} \\
\mathrm{MPa}\end{array}$ & $\rho$ & $\begin{array}{c}\mathrm{f}_{\mathrm{y}} \\
\mathrm{MPa}\end{array}$ \\
\hline L1 & 24.8 & 157 & 0.04 & 0.0142 & 362 & 0.0032 & 325 \\
\hline L2 & 24.8 & 157 & 0.04 & 0.0142 & 362 & 0.0032 & 325 \\
\hline L3 & 24.8 & 157 & 0.04 & 0.0142 & 362 & 0.0032 & 325 \\
\hline
\end{tabular}

Longitudinal steel - eight deformed bars, $19 \mathrm{~mm}$ dia.

${ }^{*}$ Calculated from data given in report

Height of specimen, $\mathrm{L}=1.6 \mathrm{~m}$

Data Files:

OHNO84L1.WK1

OHNO84L2.WK1

OHNO84L3.WK1 

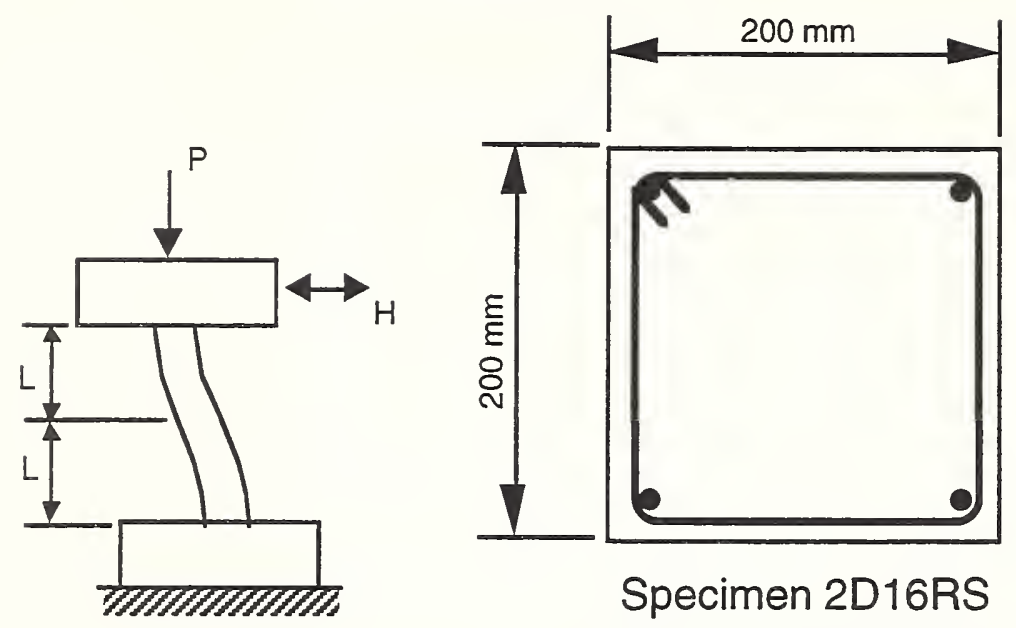

(Hoop corner detail unkown, 135 degree hooks assumed)

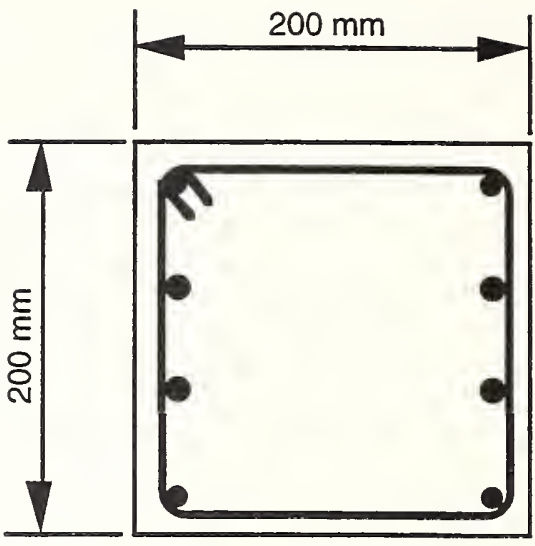

Specimen 4D13RS (Load Direction $\longrightarrow$ )

(Hoop corner detail unkown, 135 degree hooks assumed)

Ohue, Minoru; Morimoto, Hisao, Fujii, Shigeru; and Morita, Shiro, "The Behavior of R.C. Short Columns Failing in Splitting Bond-Shear Under Dynamic Lateral Loading," Transactions of the Japan Concrete Institute, Vol. 7, 1985, pp. 293-300.

Eleven double curvature columns were tested under constant axial load and cyclic lateral load. The main variable studied was the rate of lateral loading. Digitizable analog plots were available for only two of the tests in which quasi-static lateral load was applied: specimens 2D16RS and 4D13RS.

\begin{tabular}{|c|c|c|}
\hline \multirow{2}{*}{$\begin{array}{c}\text { Unit } \\
\text { No. }\end{array}$} & \multicolumn{2}{|c|}{ Lateral Reinforcement } \\
\cline { 2 - 3 } & $\begin{array}{c}\text { Bar dia. } \\
\mathrm{mm}\end{array}$ & $\begin{array}{c}\text { Spacing } \\
\text { (ctrs.) } \mathrm{mm}\end{array}$ \\
\hline 2D16RS & 6 & 50 \\
\hline 4D13RS & 6 & 50 \\
\hline
\end{tabular}

\begin{tabular}{|c|c|c|c|c|c|c|c|}
\hline $\begin{array}{c}\text { Unit } \\
\text { No. }\end{array}$ & $\begin{array}{c}\text { Concrete } \\
\text { Strength, } \\
\mathrm{MPa}\end{array}$ & $\begin{array}{c}\text { Axial } \\
\mathrm{kN}\end{array}$ & \multirow{2}{*}{$\begin{array}{c}\text { Axial Load } \\
\text { Axial Capacity* }\end{array}$} & \multicolumn{2}{|c|}{$\begin{array}{c}\text { Longitudinal } \\
\text { Reinforcement }\end{array}$} & \multicolumn{2}{|c|}{$\begin{array}{c}\text { Transverse } \\
\text { Reinforcement }\end{array}$} \\
\cline { 5 - 9 } & & & & $\rho^{*}$ & $\begin{array}{c}\mathrm{f}_{\mathrm{y}} \\
\mathrm{MPa}\end{array}$ & $\rho$ & $\begin{array}{c}\mathrm{f}_{\mathrm{y}} \\
\mathrm{MPa}\end{array}$ \\
\hline 2D16RS & 32.0 & 183 & 0.14 & 0.0201 & 369 & 0.0057 & 316 \\
\hline 4D13RS & 29.9 & 183 & 0.15 & 0.0265 & 370 & 0.0057 & 316 \\
\hline
\end{tabular}

Longitudinal steel - deformed bars, $16 \mathrm{~mm}$ dia for 2D16RS and $13 \mathrm{~mm}$ dia. for 4D13RS Lateral reinforcement is plain bars (not deformed)

${ }^{*}$ Calculated from data given in report

Half height of specimen, $\mathrm{L}=0.40 \mathrm{~m}$

Data Files:

OH2D16RS.WK1 OH4D13RS.WK1 

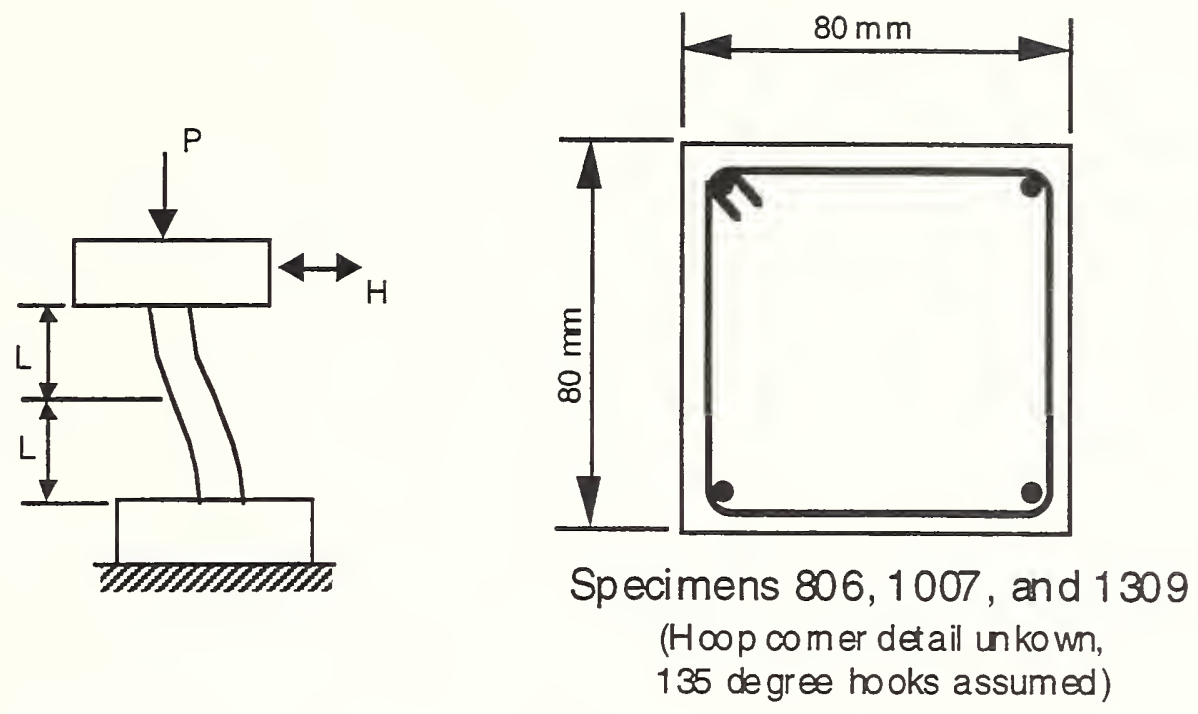

Zhou, Xiaozhen; Higashi, Yoichi; Jiang, Weishan; and Shimizu, Yasushi, "Behavior of Reinforced Concrete Column Under High Axial Load," Transactions of the Japan Concrete Institute, Vol. 7, 1985, pp. 385-392.

Seventeen one-fifth scale, double curvature column specimens were tested. This study focuses on short columns with high axial loads. Only three digitizable analog plots were available from the paper: specimens 806, 1007, and 1309

\begin{tabular}{|c|c|c|}
\hline \multirow{2}{*}{$\begin{array}{l}\text { Unit } \\
\text { No. }\end{array}$} & \multicolumn{2}{|c|}{ Lateral Reinforcement } \\
\cline { 2 - 3 } & $\begin{array}{c}\text { Bar dia. } \\
\text { mm }\end{array}$ & $\begin{array}{c}\text { Spacing } \\
\text { (ctrs.) mm }\end{array}$ \\
\hline 806 & 4 & 80 \\
\hline 1007 & 4 & 80 \\
\hline 1309 & 4 & 80 \\
\hline
\end{tabular}

\begin{tabular}{|c|c|c|c|c|c|c|c|}
\hline \multirow{2}{*}{$\begin{array}{c}\text { Unit } \\
\text { No. }\end{array}$} & $\begin{array}{c}\text { Concrete } \\
\text { Strength, } \\
\text { MPa }\end{array}$ & $\begin{array}{c}\text { Axial } \\
\text { Load } \\
\mathrm{kN}\end{array}$ & \multirow{2}{*}{$\begin{array}{c}\text { Axial Load } \\
\text { Axial Capacity* }\end{array}$} & \multicolumn{2}{|c|}{$\begin{array}{c}\text { Longitudinal } \\
\text { Reinforcement }\end{array}$} & \multicolumn{2}{|c|}{$\begin{array}{c}\text { Transverse } \\
\text { Reinforcement }\end{array}$} \\
\cline { 5 - 8 } & & & & $\rho^{*}$ & $\begin{array}{c}\mathrm{f}_{\mathrm{y}} \\
\text { MPa }\end{array}$ & $\rho *$ & $\begin{array}{c}\mathrm{f}_{\mathrm{y}} \\
\mathrm{MPa}\end{array}$ \\
\hline 806 & 32.3 & 124 & 0.6 & 0.0177 & 336 & 0.0052 & 341 \\
\hline 1007 & 34.0 & 152 & 0.7 & 0.0177 & 336 & 0.0052 & 341 \\
\hline 1309 & 32.8 & 189 & 0.9 & 0.0177 & 336 & 0.0052 & 341 \\
\hline
\end{tabular}

Longitudinal steel - four deformed bars, $6 \mathrm{~mm}$ dia

Lateral reinforcement is plain bars (not deformed)

${ }^{*}$ Calculated from data given in report

Half height of specimen, $\mathrm{L}=0.08 \mathrm{~m}$

Data Files:

ZHO806.WK1

ZHO1007.WK1

ZHO1309.WK1 

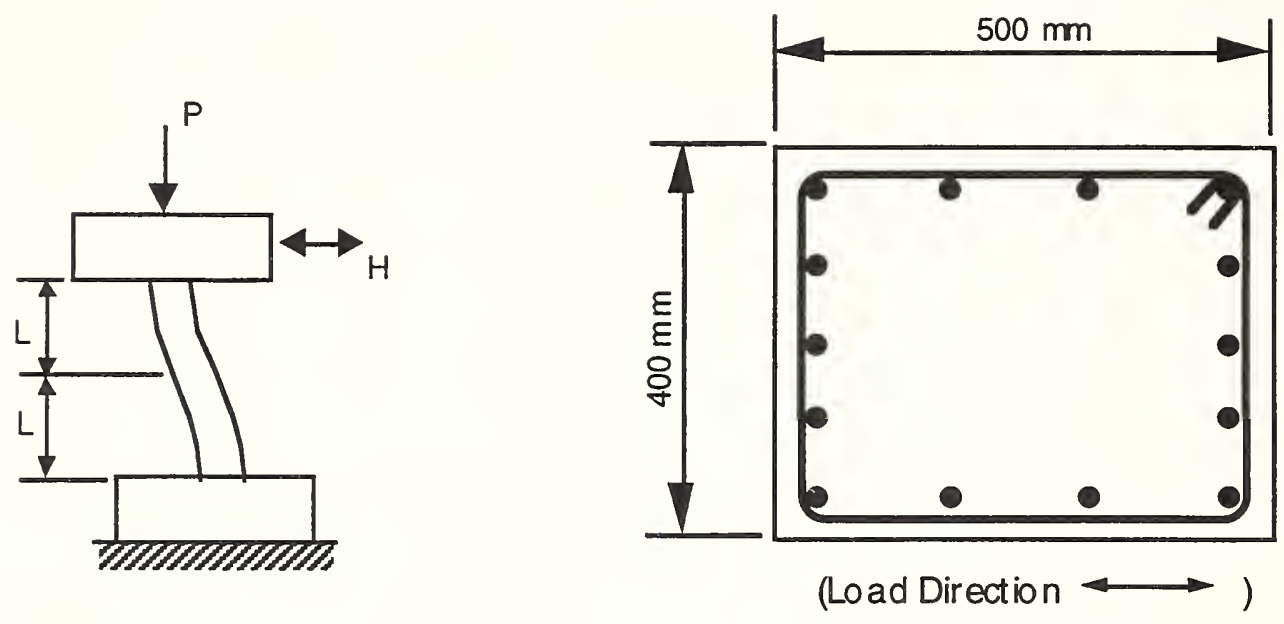

(Hoop corner detail unkown $135 \mathrm{dgree} \mathrm{hooks} \mathrm{assumed)}$

Imai, Hiroshi; and Yamamoto, Yoshie, "A Study on Causes of Earthquake Damage of Izumi High School Due to Miyagi-Ken-Oki Earthquake in 1978," Transactions of the Japan Concrete Institute, Vol. 8, 1986, pp. 405-418.

One full-size double curvature specimen was tested. The specimen simulated columns in a high school building damaged during the 1978 Miyagi-ken-oki earthquake

\begin{tabular}{|c|c|c|}
\hline \multirow{2}{*}{$\begin{array}{l}\text { Unit } \\
\text { No. }\end{array}$} & \multicolumn{2}{|c|}{ Lateral Reinforcement } \\
\hline & $\begin{array}{c}\text { Bar dia. } \\
\text { mm }\end{array}$ & $\begin{array}{c}\text { Spacing } \\
\text { (ctrs.) } \mathrm{mm}\end{array}$ \\
\hline 1 & 9 & 100 \\
\hline
\end{tabular}

\begin{tabular}{|c|c|c|c|c|c|c|c|}
\hline \multirow{2}{*}{$\begin{array}{c}\text { Unit } \\
\text { No. }\end{array}$} & $\begin{array}{c}\text { Concrete } \\
\text { Strength, } \\
\mathrm{MPa}\end{array}$ & $\begin{array}{c}\text { Axial } \\
\text { Load } \\
\mathrm{kN}\end{array}$ & \multirow{2}{*}{$\begin{array}{c}\text { Axial Load } \\
\text { Axial Capacity* }\end{array}$} & \multicolumn{2}{|c|}{$\begin{array}{c}\text { Longitudinal } \\
\text { Reinforcement }\end{array}$} & \multicolumn{2}{|c|}{$\begin{array}{c}\text { Transverse } \\
\text { Reinforcement }\end{array}$} \\
\cline { 5 - 8 } & & & $\rho *$ & $\begin{array}{c}\mathrm{f}_{\mathrm{y}} \\
\mathrm{MPa}\end{array}$ & $\rho * *$ & $\begin{array}{c}\mathrm{f}_{\mathrm{y}} \\
\mathrm{MPa}\end{array}$ \\
\hline 1 & 27.1 & 392 & 0.072 & 0.0209 & 318 & 0.0036 & 336 \\
\hline
\end{tabular}

Longitudinal steel - 14 deformed bars, $22 \mathrm{~mm}$ dia

${ }^{*}$ Calculated from data given in report

**Approximate value estimated from data given in report

Half height of specimen, $\mathrm{L}=0.825 \mathrm{~m}$

Data Files:

IMAI86.WK1 


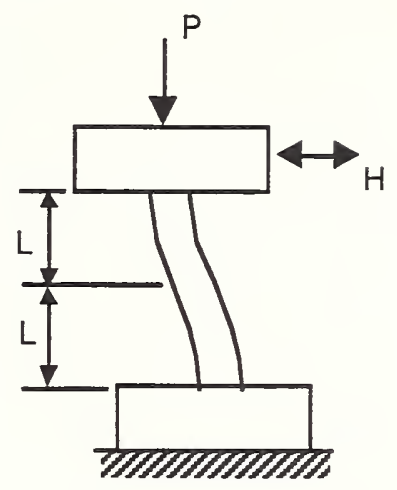

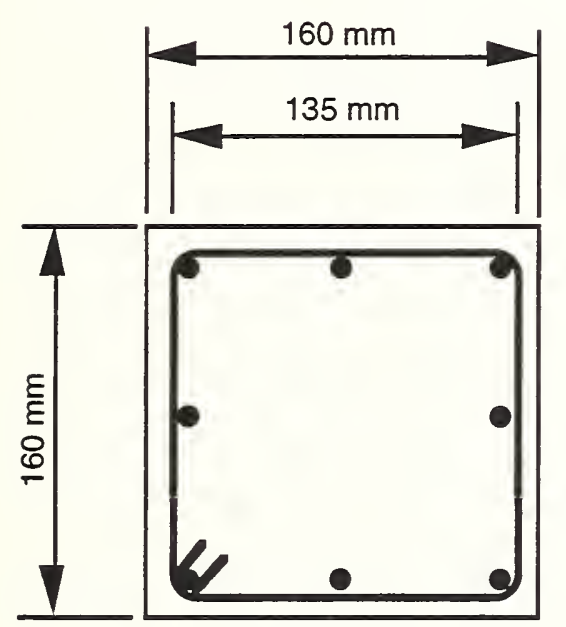

Specimens 104-08, 204-08 and $302-07$

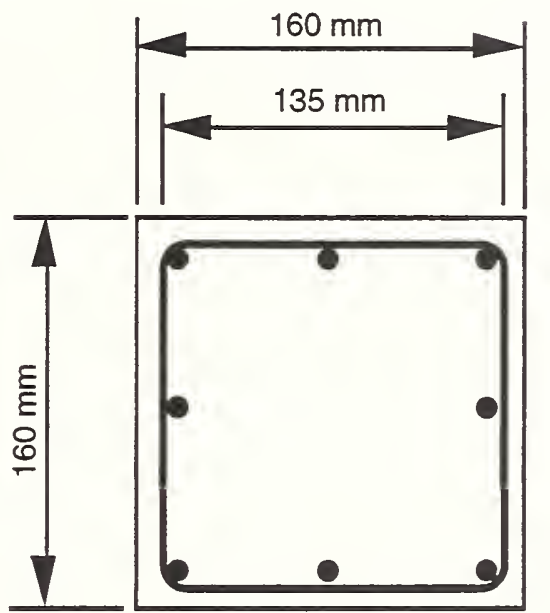

Spiral hoops Specimens 114-08, 214-08 and 312-07

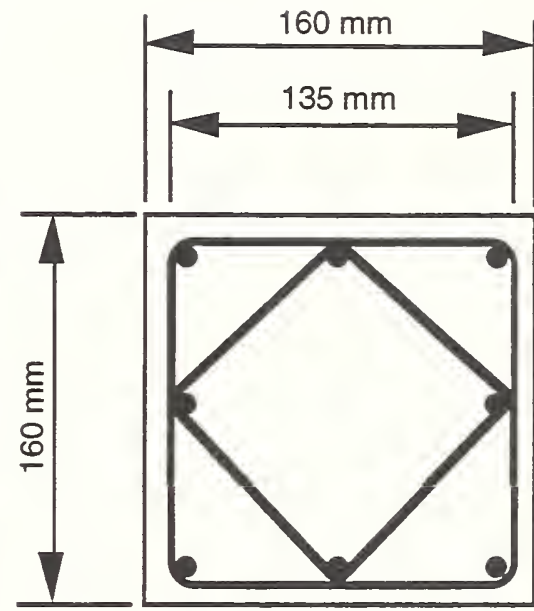

Double spiral hoops Specimens

124-08, 223-09 and 322-07

Zhou, Xiaoshen; Satoh, Toshio; Jiang, Weishan; Ono, Arata; and Shimizo, Yasushi, "Behavior of Reinforced Concrete Short Column Under High Axial Load," Transactions of the Japan Concrete Institute, Vol. 9, 1987, pp. 541-548.

Thirty-five double curvature column specimens, at approximately $2 / 5$ scale, were tested under constant axial load and cyclic lateral load. The main objective of the test series was to investigate the seismic performance of columns under high compressive stress. Digitizable analog plots from only nine specimens were available in the paper. 


\begin{tabular}{|c|c|c|}
\hline \multirow{2}{*}{\begin{tabular}{l} 
Nnit \\
\cline { 2 - 3 }
\end{tabular}} & $\begin{array}{c}\text { Lateral Reinforcement } \\
\text { Bar dia. } \\
\text { mm }\end{array}$ & $\begin{array}{c}\text { Spacing } \\
\text { (ctrs.) mm }\end{array}$ \\
\hline $104-08$ & 5 & 40 \\
\hline $114-08$ & 5 & 40 \\
\hline $124-08$ & 5 & 40 \\
\hline $204-08$ & 5 & 40 \\
\hline $214-08$ & 5 & 40 \\
\hline $223-09$ & 5 & 40 \\
\hline $302-07$ & 5 & 40 \\
\hline $312-07$ & 5 & 40 \\
\hline $322-07$ & 5 & 40 \\
\hline
\end{tabular}

\begin{tabular}{|c|c|c|c|c|c|c|c|}
\hline \multirow{2}{*}{$\begin{array}{c}\text { Unit } \\
\text { No. }\end{array}$} & $\begin{array}{c}\text { Soncrete } \\
\text { Strength, } \\
\mathrm{MPa}\end{array}$ & $\begin{array}{c}\text { Axial } \\
\text { Load } \\
\mathrm{kN}\end{array}$ & \multirow{2}{*}{$\begin{array}{c}\text { Axial Capacity* } \\
\text { Axial Capad }\end{array}$} & \multicolumn{2}{|c|}{$\begin{array}{c}\text { Longitudinal } \\
\text { Reinforcement }\end{array}$} & \multicolumn{2}{|c|}{$\begin{array}{c}\text { Transverse } \\
\text { Reinforcement }\end{array}$} \\
\cline { 5 - 8 } & & & & $\rho^{*}$ & $\begin{array}{c}\mathrm{f}_{\mathrm{y}} \\
\mathrm{MPa}\end{array}$ & $\rho^{*}$ & $\begin{array}{c}\mathrm{f}_{\mathrm{y}} \\
\mathrm{MPa}\end{array}$ \\
\hline $104-08$ & 19.8 & 406 & 0.8 & 2.45 & 341 & 0.0073 & 559 \\
\hline $114-08$ & 19.8 & 406 & 0.8 & 2.45 & 341 & 0.0073 & 559 \\
\hline $124-08$ & 19.8 & 406 & 0.8 & 2.45 & 341 & 0.0175 & 559 \\
\hline $204-08$ & 21.1 & 432 & 0.8 & 2.45 & 341 & 0.0073 & 559 \\
\hline $214-08$ & 21.1 & 432 & 0.8 & 2.45 & 341 & 0.0073 & 559 \\
\hline $223-09$ & 21.1 & 486 & 0.9 & 2.45 & 341 & 0.0175 & 559 \\
\hline $302-07$ & 28.8 & 517 & 0.7 & 2.45 & 341 & 0.0073 & 559 \\
\hline $312-07$ & 28.8 & 517 & 0.7 & 2.45 & 341 & 0.0073 & 559 \\
\hline $322-07$ & 28.8 & 517 & 0.7 & 2.45 & 341 & 0.0175 & 559 \\
\hline
\end{tabular}

Longitudinal steel - eight bars, $10 \mathrm{~mm}$ dia

* Calculated from data given in report

Half height of specimens: $\quad 104-08,114-08$ and $124-08, \mathrm{~L}=0.16 \mathrm{~m}$ 204-08, 214-08 and 223-09, $\mathrm{L}=0.32 \mathrm{~m}$ $302-07,312-07$ and $322-07, \mathrm{~L}=0.48 \mathrm{~m}$

Data Files:

ZHO10408.WK1
ZHO1 1408.WK1
ZHO12408.WK1
ZHO20408.WK1
ZHO21408.WK1
ZHO22309.WK1
ZHO30207.WK1
ZHO31207.WK1
ZHO32207.WK1




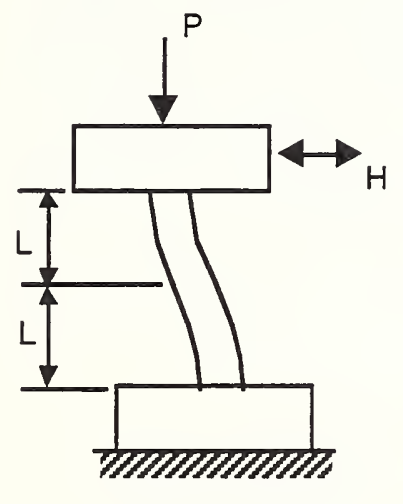

Kanda, Makoto; Shirai, Nobuaki; Adachi, Hiromi; and Sato, Toshio, "Analytical Study on Elasto-Plastic Hysteretic Behaviors of Reinforced Concrete Members," Transactions of the Japan Concrete Institute, Vol. 10, 1988, pp. 257-264. (Specimens 85STC-3 and 85PDC-3 only)

Six double curvature specimens were tested. In three of the tests ("STC" series) pseudostatic cyclic loads were applied in the form of a sawtooth wave with gradually increasing amplitude. In the remaining three tests ("PDC" series) a pseudo-dynamic testing procedure was followed, to simulate earthquake loading of the columns. The three specimens in each series had different details for anchorage of the longitudinal bars. The influence of the anchorage detail is not discussed in the paper by Kanda et al. because the paper mainly focuses on finite element modeling methods. Digital files of load-deflection data for all six tests were obtained directly from the researchers who performed the tests.

\begin{tabular}{|c|c|c|}
\hline \multirow{2}{*}{$\begin{array}{c}\text { Unit } \\
\text { No. }\end{array}$} & \multicolumn{2}{|c|}{ Lateral Reinforcement } \\
\cline { 2 - 3 } & $\begin{array}{c}\text { Bardia. } \\
\text { mm }\end{array}$ & $\begin{array}{c}\text { Spacing } \\
\text { (ctrs.) } \mathrm{mm}\end{array}$ \\
\hline 85STC-1 & 6 & 50 \\
\hline 85STC-2 & 6 & 50 \\
\hline 85STC-3 & 6 & 50 \\
\hline 85PDC-1 & 6 & 50 \\
\hline 85PDC-2 & 6 & 50 \\
\hline 85PDC-3 & 6 & 50 \\
\hline
\end{tabular}




\begin{tabular}{|c|c|c|c|c|c|c|c|}
\hline $\begin{array}{c}\text { Unit } \\
\text { No. }\end{array}$ & $\begin{array}{c}\text { Concrete } \\
\text { Strength, } \\
\text { MPa }\end{array}$ & $\begin{array}{c}\text { Axial } \\
\text { Load } \\
\text { kN* }\end{array}$ & \multirow{2}{*}{$\begin{array}{c}\text { Axial Load } \\
\text { Axial Capacity* }\end{array}$} & \multicolumn{2}{|c|}{$\begin{array}{c}\text { Longitudinal } \\
\text { Reinforcement }\end{array}$} & \multicolumn{2}{|c|}{$\begin{array}{c}\text { Transverse } \\
\text { Reinforcement }\end{array}$} \\
\cline { 5 - 8 } & & & & $\rho *$ & $\begin{array}{c}\mathrm{f}_{\mathrm{y}} \\
\mathrm{MPa}^{*}\end{array}$ & $\rho *$ & $\begin{array}{c}\mathrm{f}_{\mathrm{y}} \\
\mathrm{MPa}\end{array}$ \\
\hline 85STC-1 & 27.9 & $183.9^{* *}$ & 0.088 & 0.0142 & 374 & 0.0038 & 506 \\
\hline 85STC-2 & 27.9 & $183.9^{* *}$ & 0.088 & 0.0142 & 374 & 0.0038 & 506 \\
\hline 85STC-3 & 27.9 & 183.9 & 0.088 & 0.0142 & 374 & 0.0038 & 506 \\
\hline 85PDC-1 & 24.8 & $183.9^{* *}$ & 0.099 & 0.0142 & 338 & 0.0038 & 352 \\
\hline 85PDC-2 & 27.9 & $183.9^{* *}$ & 0.088 & 0.0142 & 374 & 0.0038 & 506 \\
\hline 85PDC-3 & 27.9 & 183.9 & 0.088 & 0.0142 & 374 & 0.0038 & 506 \\
\hline
\end{tabular}

Longitudinal steel - eight deformed bars, $13 \mathrm{~mm}$ diameter

Lateral reinforcement - plain bars, $6 \mathrm{~mm}$ dia., except deformed bars $6 \mathrm{~mm}$ dia for 85PDC-1

${ }^{*}$ Calculated from data given in report

**Assumed value, inferred from report

Half height of specimens: $\mathrm{L}=750 \mathrm{~mm}$

Data Files:

KANSTC1.WK1

KANSTC2.WK1

KANSTC3.WK1

KANPDC1.WK1

KANPDC2.WK1

KANPDC3.WK1 


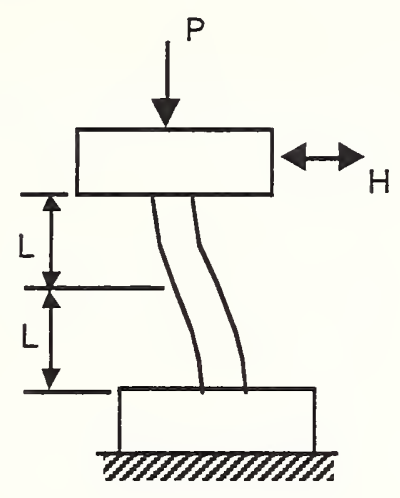

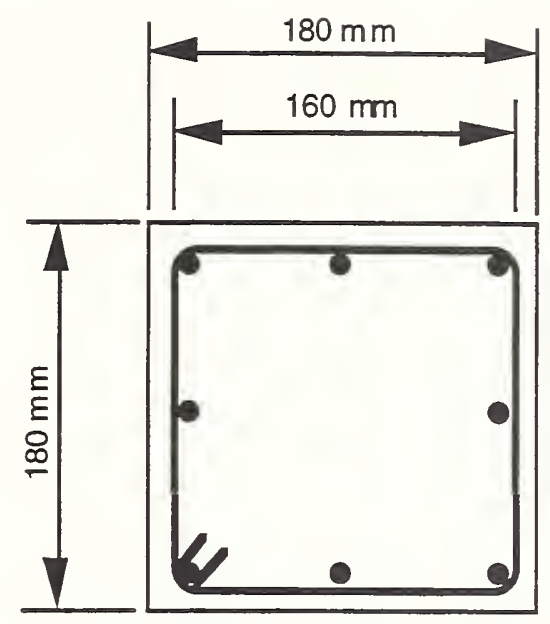

Specimens $\mathrm{OA} 2$ and $\mathrm{OA} 5$

Arakawa, Takashi; Arai, Yasuyuki; Mizoguchi, Mitsuo; and Yoshida, Minoru, "Shear Resisting Behavior of Short Reinforced Concrete Columns Under Biaxial Bending-Shear," Transactions of the Japan Concrete Institute, Vol. 11, 1989, pp. 317-324.

Fourteen double-curvature specimens were tested. The main variables studied were the direction of loading $(0,22.5$, or 45 degrees, with respect to the principal axes of the cross section), and the level of axial compression or tension. Two plots were digitized from tests in which the lateral loads were applied at 0 degrees to the principal axes, and axial compression load was applied.

\begin{tabular}{|c|c|c|}
\hline \multirow{2}{*}{$\begin{array}{l}\text { Unit } \\
\text { No. }\end{array}$} & \multicolumn{2}{|c|}{ Lateral Reinforcement } \\
\hline & $\begin{array}{c}\text { Bar dia. } \\
\text { mm }\end{array}$ & $\begin{array}{c}\text { Spacing } \\
\text { (ctrs.) } \mathrm{mm}\end{array}$ \\
\hline OA2 & 4 & 64.3 \\
\hline$\overline{\mathrm{OA5}}$ & 4 & 64.3 \\
\hline
\end{tabular}

\begin{tabular}{|l|c|c|c|c|c|c|c|}
\hline \multirow{2}{*}{$\begin{array}{l}\text { Unit } \\
\text { No. }\end{array}$} & $\begin{array}{c}\text { Concrete } \\
\text { Strength, } \\
\text { MPa }\end{array}$ & $\begin{array}{c}\text { Axial } \\
\text { Load } \\
\mathrm{kN}^{*}\end{array}$ & \multirow{2}{*}{ Axial Capacity* } & \multicolumn{2}{|c|}{$\begin{array}{c}\text { Longitudinal } \\
\text { Reinforcement }\end{array}$} & \multicolumn{2}{|c|}{$\begin{array}{c}\text { Transverse } \\
\text { Reinforcement }\end{array}$} \\
\cline { 5 - 8 } & & & & $\rho *$ & $\begin{array}{c}\mathrm{f}_{\mathrm{y}} \\
\mathrm{MPa}\end{array}$ & $\rho^{*}$ & $\begin{array}{c}\mathrm{f}_{\mathrm{y}} \\
\mathrm{MPa}\end{array}$ \\
\hline OA2 & 31.8 & 190 & 0.185 & 0.0313 & 340 & 0.0024 & 249 \\
\hline OA5 & 33.0 & 476 & 0.445 & 0.0313 & 340 & 0.0024 & 249 \\
\hline
\end{tabular}

Longitudinal steel - eight deformed bars, $13 \mathrm{~mm}$ dia

${ }^{*}$ Calculated from data given in report

Half height of specimens: $\mathrm{L}=225 \mathrm{~mm}$

Data Files:

ARA89OA2.WK1

ARA89OA5.WK1 


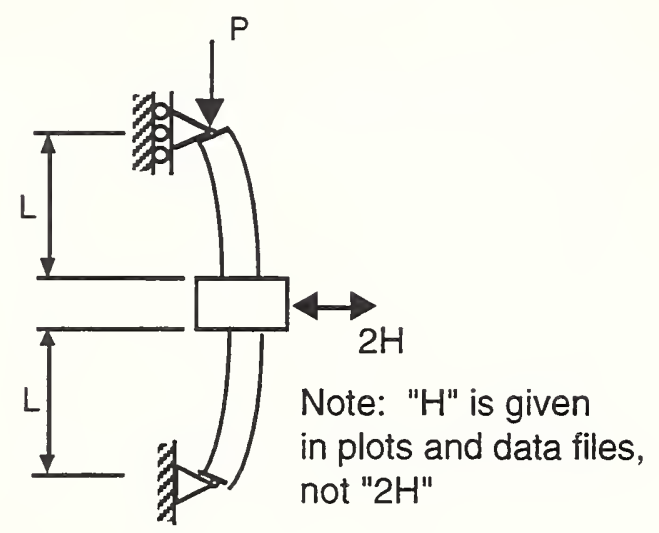

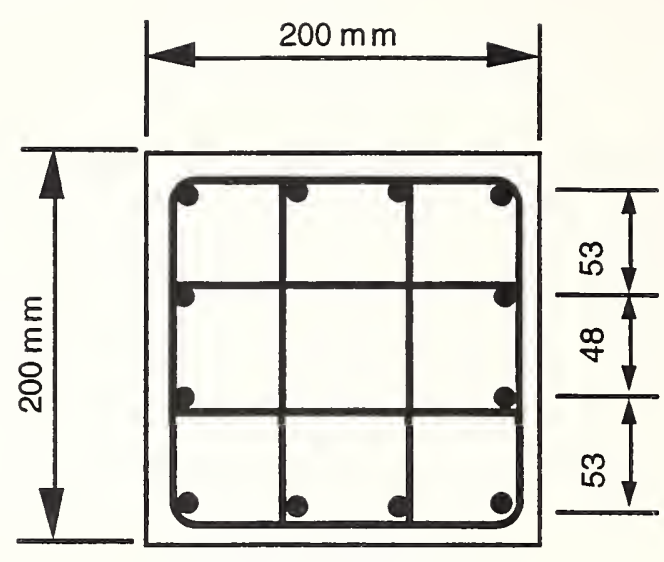

All Specimens

(Hoops and ties are

welded closed)

Muguruma, Hiroshi; Watanabe, Fumio; and Komuro, Tsutomu, "Applicability of High Strength Concrete to Reinforced Concrete Ductile Column," Transactions of the Japan Concrete Institute, Vol. 11, 1989, pp. 309-316.

Eight columns were tested. The tests focused on high-strength concrete in combination with high-strength lateral reinforcement. The main variable studied were the compressive strength of the concrete, the axial load level, and the yield strength of the confining reinforcement. Doubleended specimens were tested. A self-reacting load frame with hydraulic ram to apply cyclic lateral load and a universal testing machine to apply constant axial load were used. Digital load-deflection data were obtained directly from the researchers who performed the study.

\begin{tabular}{|c|c|c|}
\hline \multirow{2}{*}{$\begin{array}{l}\text { Unit } \\
\text { No. }\end{array}$} & \multicolumn{2}{|c|}{ Lateral Reinforcement } \\
\hline & $\begin{array}{c}\text { Bar dia. } \\
\mathrm{mm}\end{array}$ & $\begin{array}{l}\text { Spacing } \\
\text { (ctrs.) } \mathrm{mm}\end{array}$ \\
\hline AL-1 & 6 & 35 \\
\hline$\overline{\mathrm{AH}-1}$ & 6 & 35 \\
\hline$\overline{\mathrm{AL}-2}$ & 6 & 35 \\
\hline$\overline{\mathrm{AH}}-2$ & 6 & 35 \\
\hline$\overline{\mathrm{BL}-1}$ & 6 & 35 \\
\hline$\overline{\mathrm{BH}-1}$ & 6 & 35 \\
\hline$\overline{\mathrm{BL}-2}$ & 6 & 35 \\
\hline$\overline{\mathrm{BH}-2}$ & 6 & 35 \\
\hline
\end{tabular}




\begin{tabular}{|c|c|c|c|c|c|c|c|}
\hline $\begin{array}{c}\text { Unit } \\
\text { No. }\end{array}$ & $\begin{array}{c}\text { Concrete } \\
\text { Strength, } \\
\text { MPa }\end{array}$ & $\begin{array}{c}\text { Axial } \\
\text { Load } \\
\mathrm{kN}\end{array}$ & \multirow{2}{*}{$\begin{array}{c}\text { Axial Load } \\
\text { Axial Capacity }\end{array}$} & \multicolumn{2}{|c|}{$\begin{array}{c}\text { Longitudinal } \\
\text { Reinforcement }\end{array}$} & \multicolumn{2}{|c|}{$\begin{array}{c}\text { Transverse } \\
\text { Reinforcement }\end{array}$} \\
\cline { 5 - 8 } & & & & $\rho$ & $\begin{array}{c}\mathrm{f}_{\mathrm{y}} \\
\mathrm{MPa}\end{array}$ & $\rho$ & $\begin{array}{c}\mathrm{f}_{\mathrm{y}} \\
\mathrm{MPa}\end{array}$ \\
\hline AL-1 & 85.7 & 1371 & 0.400 & 0.0381 & 399.6 & 0.0161 & 328.4 \\
\hline AH-1 & 85.7 & 1371 & 0.400 & 0.0381 & 399.6 & 0.0161 & 792.3 \\
\hline AL-2 & 85.7 & 2156 & 0.629 & 0.0381 & 399.6 & 0.0161 & 328.4 \\
\hline AH-2 & 85.7 & 2156 & 0.629 & 0.0381 & 399.6 & 0.0161 & 792.3 \\
\hline BL-1 & 115.8 & 1176 & 0.254 & 0.0381 & 399.6 & 0.0161 & 328.4 \\
\hline BH-1 & 115.8 & 1176 & 0.254 & 0.0381 & 399.6 & 0.0161 & 792.3 \\
\hline BL-2 & 115.8 & 1959 & 0.423 & 0.0381 & 399.6 & 0.0161 & 328.4 \\
\hline BH-2 & 115.8 & 1959 & 0.423 & 0.0381 & 399.6 & 0.0161 & 792.3 \\
\hline
\end{tabular}

Longitudinal steel - twelve deformed bars, $13 \mathrm{~mm}$ diameter

Lateral reinforcement - plain bars, $6 \mathrm{~mm}$ in diameter

Clear cover to lateral reinforcement $=9 \mathrm{~mm}$

${ }^{*}$ Calculated from data given in report

Half height of specimens: $\mathrm{L}=500 \mathrm{~mm}$

Data Files:

MUG89AL1.WK1
MUG89AH1.WK1
MUG89AL2.WK1
MUG89AH2.WK1
MUG89BL1.WK1
MUG89BH1.WK1
MUG89BL2.WK1
MUG89BH2.WK1 

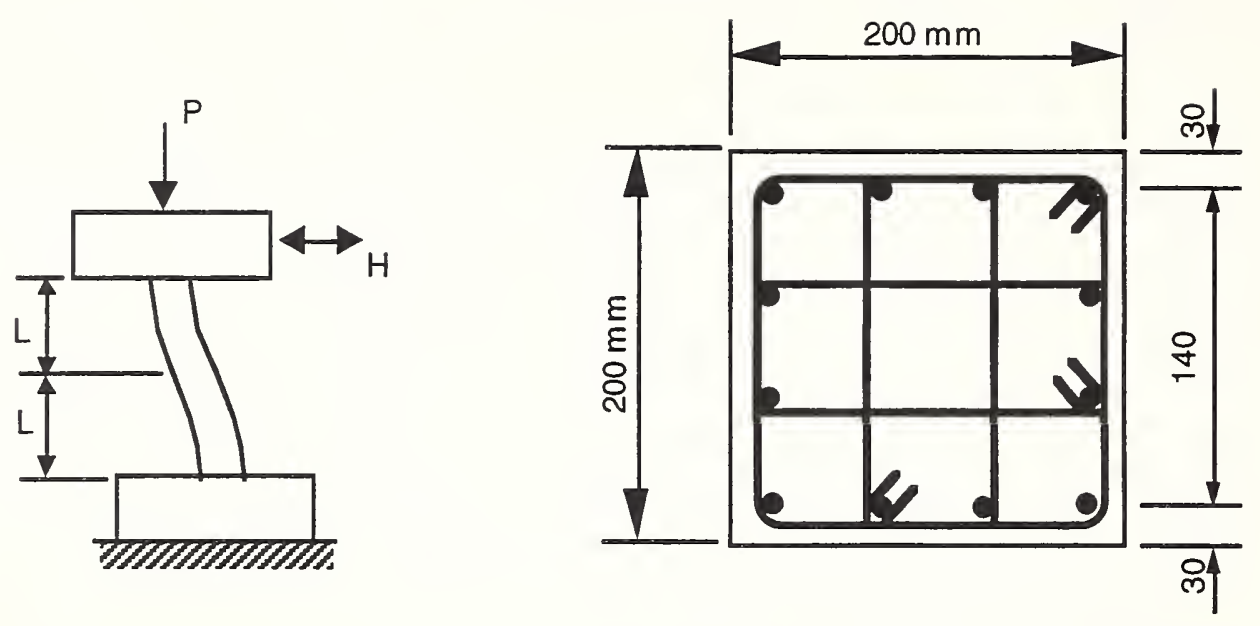

Specimens CA025C and CA060C

Ono, Arata; Shirai, Nobuaki; Adachi, Hiromi; and Sakamaki, Yoshio, "Elasto-Plastic Behavior of Reinforced Concrete Column With Fluctuating Axial Force," Transactions of the Japan Concrete Institute, Vol. 11, 1989, pp. 239-246.

Four double curvature specimens were tested. The main variable studied in this series was the axial load level. One specimen was tested with fluctuating axial load; one was tested with axial tension; and two were tested with constant axial compression. Only results from the two tests with constant axial compression are reported here.

\begin{tabular}{|c|c|c|}
\hline \multirow{2}{*}{$\begin{array}{l}\text { Unit } \\
\text { No. }\end{array}$} & \multicolumn{2}{|c|}{ Lateral Reinforcement } \\
\hline & $\begin{array}{c}\text { Bar dia. } \\
\text { mm }\end{array}$ & $\begin{array}{l}\text { Spacing } \\
\text { (ctrs.) mm }\end{array}$ \\
\hline CA025C & 6 & 70 \\
\hline CA060C & 6 & 70 \\
\hline
\end{tabular}

\begin{tabular}{|c|c|c|c|c|c|c|c|}
\hline $\begin{array}{c}\text { Unit } \\
\text { No. }\end{array}$ & $\begin{array}{c}\text { Concrete } \\
\text { Strength, } \\
\mathrm{MPa}\end{array}$ & $\begin{array}{c}\text { Axial } \\
\text { Load } \\
\mathrm{kN}\end{array}$ & Axial Capacity* & \multicolumn{2}{|c|}{$\begin{array}{c}\text { Longitudinal } \\
\text { Reinforcement }\end{array}$} & \multicolumn{2}{|c|}{$\begin{array}{c}\text { Transverse } \\
\text { Reinforcement }\end{array}$} \\
\cline { 5 - 8 } & & & & $\rho *$ & $\begin{array}{c}\mathrm{f}_{\mathrm{y}} \\
\mathrm{MPa}\end{array}$ & $\rho$ & $\begin{array}{c}\mathrm{f}_{\mathrm{y}} \\
\mathrm{MPa}\end{array}$ \\
\hline CA025C & 25.8 & 265 & 0.25 & 0.0236 & 361 & 0.0091 & 426 \\
\hline CA060C & 25.8 & 636 & 0.60 & 0.0236 & 361 & 0.0091 & 426 \\
\hline
\end{tabular}

Longitudinal steel - twelve deformed bars, $10 \mathrm{~mm}$ dia

* Calculated from data given in report

Half height of specimens: $\mathrm{L}=300 \mathrm{~mm}$

Data Files:

ONO025C.WK1

ONO060C.WK1 


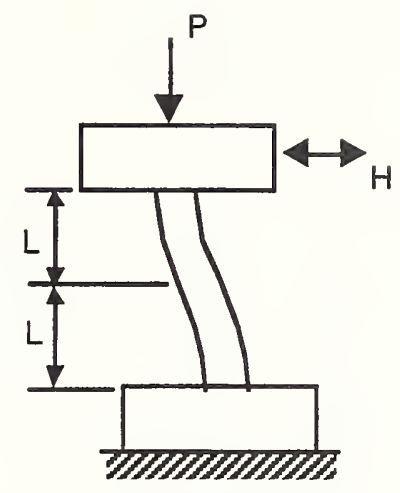

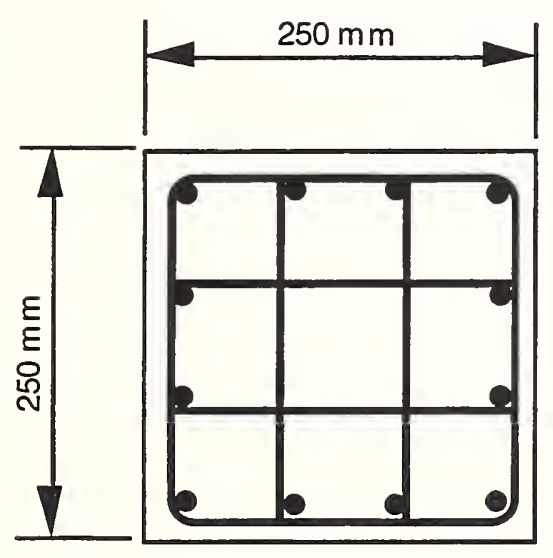

Specimens 1, 2, 3, and 4

(Hoops are welded)
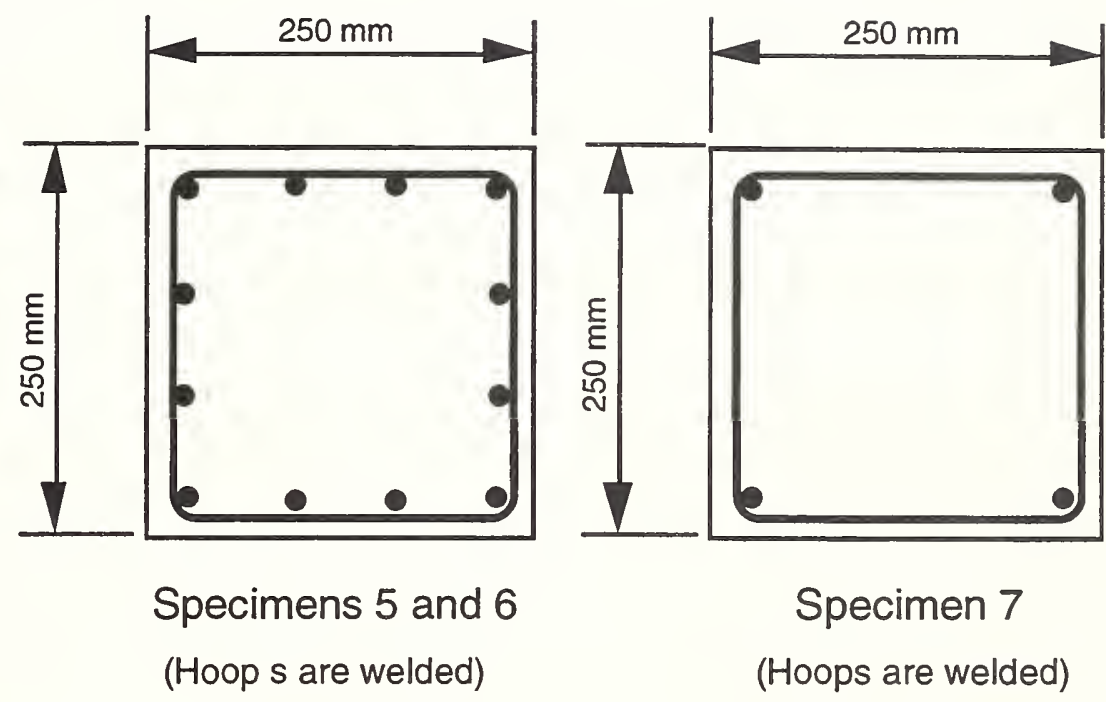

Sakai, Yuuki; Hibi, Junichi; Otani, Shunsuke; and Aoyama, Hiroyuki, "Experimental Study on Flexural Behavior of Reinforced Concrete Columns Using High-Strength Concrete," Transactions of the Japan Concrete Institute, Vol. 12, 1990, pp. 323-330.

The main purpose of this study was to investigate the behavior of columns made of high strength concrete. Eight double curvature specimens were tested. Digital data files were obtained directly from the authors. The axial load applied to Specimen B8 was not constant, so results from B8 are not presented here.

\begin{tabular}{|c|c|c|}
\hline \multirow{2}{*}{$\begin{array}{l}\text { Unit } \\
\text { No. }\end{array}$} & \multicolumn{2}{|c|}{ Lateral Reinforcement } \\
\hline & $\begin{array}{l}\text { Bar dia. } \\
\text { mm }\end{array}$ & $\begin{array}{l}\text { Spacing } \\
\text { (ctrs.) } \mathrm{mm}\end{array}$ \\
\hline$\overline{\mathrm{B} 1}$ & 5 & 60 \\
\hline$\overline{\mathrm{B} 2}$ & 5 & 40 \\
\hline$\overline{\mathrm{B} 3}$ & 5.5 & 60 \\
\hline$\overline{\mathrm{B} 4}$ & 5 & 60 \\
\hline $\mathrm{B} 5$ & 5 & 30 \\
\hline$\overline{\mathrm{B} 6}$ & 7 & 60 \\
\hline$\overline{B 7}$ & 5 & 30 \\
\hline
\end{tabular}




\begin{tabular}{|c|c|c|c|c|c|c|c|}
\hline \multirow{2}{*}{$\begin{array}{c}\text { Unit } \\
\text { No. }\end{array}$} & $\begin{array}{c}\text { Concrete } \\
\text { Strength, } \\
\text { MPa }\end{array}$ & $\begin{array}{c}\text { Axial } \\
\text { Load } \\
\mathrm{kN}\end{array}$ & \multirow{2}{*}{$\begin{array}{c}\text { Axial Load } \\
\text { Axial Capacity* }\end{array}$} & \multicolumn{2}{|c|}{$\begin{array}{c}\text { Longitudinal } \\
\text { Reinforcement }\end{array}$} & \multicolumn{2}{|c|}{$\begin{array}{c}\text { Transverse } \\
\text { Reinforcement }\end{array}$} \\
\cline { 5 - 8 } & & & & $\rho^{*}$ & $\begin{array}{c}\mathrm{f}_{\mathrm{y}} \\
\mathrm{MPa}_{\mathrm{n}}\end{array}$ & $\rho$ & $\begin{array}{c}\mathrm{f}_{\mathrm{y}} \\
\mathrm{MPa}\end{array}$ \\
\hline B1 & 99.5 & 2176 & 0.35 & 0.0255 & 379 & 0.0050 & 774 \\
\hline B2 & 99.5 & 2176 & 0.35 & 0.0255 & 379 & 0.0075 & 774 \\
\hline B3 & 99.5 & 2176 & 0.35 & 0.0255 & 379 & 0.0061 & 344 \\
\hline B4 & 99.5 & 2176 & 0.35 & 0.0255 & 379 & 0.0050 & 1126 \\
\hline B5 & 99.5 & 2176 & 0.35 & 0.0255 & 379 & 0.0050 & 774 \\
\hline B6 & 99.5 & 2176 & 0.35 & 0.0255 & 379 & 0.0050 & 857 \\
\hline B7 & 99.5 & 2176 & 0.35 & 0.0181 & 339 & 0.0050 & 774 \\
\hline
\end{tabular}

Longitudinal steel - Specimens B1 to B6, twelve $13 \mathrm{~mm}$ diameter deformed bars Specimen B7, four $19 \mathrm{~mm}$ diameter deformed bars

* Calculated from data given in report

Half height of specimens: $\mathrm{L}=500 \mathrm{~mm}$

Data Files:

SAK90B1.WK1

SAK90B2.WK1

SAK90B3.WK1

SAK90B4.WK1

SAK90B5.WK1

SAK90B6.WK1

SAK90B7.WK1 

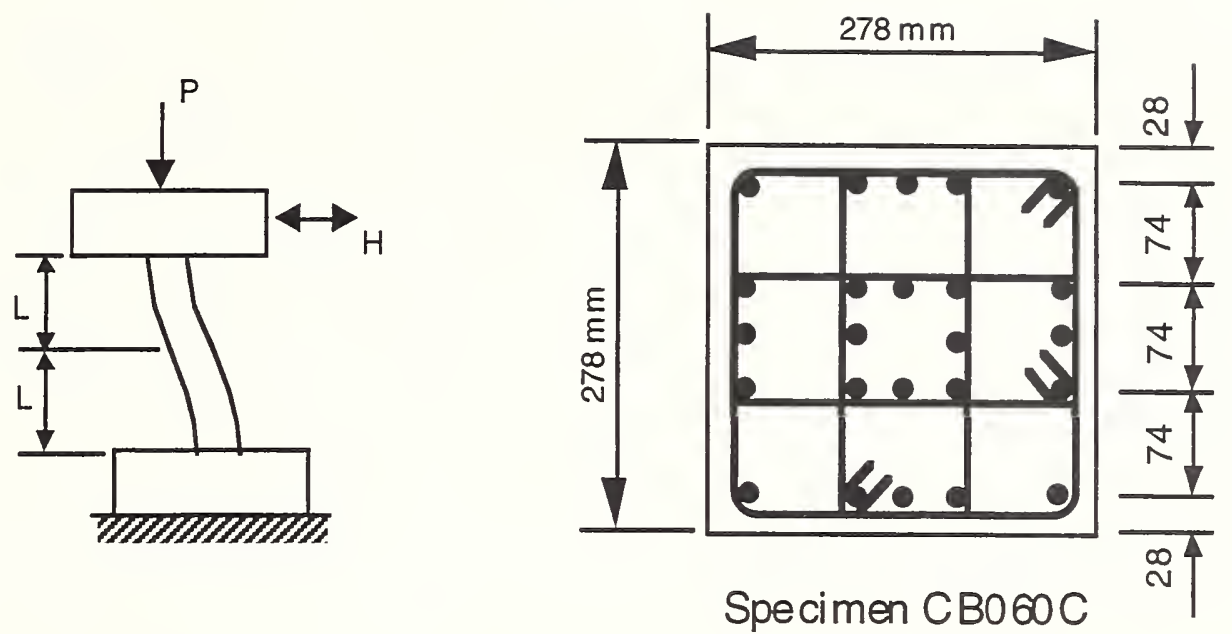

Amitsu, Shigeyuki; Shirai, Nobuaki; Adachi, Hiromi; and Ono, Arata, "Deformation of Reinforced Concrete Column with High or Fluctuating Axial Force," Transactions of the Japan Concrete Institute, Vol. 13, 1991, pp. 355-362.

Three double curvature specimens were tested at $1 / 3.25$ scale. Only one specimen was tested with a constant axial force. As shown in the sketch above, the configuration of the longitudinal reinforcement was unusual.

\begin{tabular}{|c|c|c|}
\hline \multirow{2}{*}{$\begin{array}{l}\text { Unit } \\
\text { No. }\end{array}$} & \multicolumn{2}{|c|}{ Lateral Reinforcement } \\
\cline { 2 - 3 } & $\begin{array}{c}\text { Bar dia. } \\
\mathrm{mm}\end{array}$ & $\begin{array}{c}\text { Spacing } \\
\text { (ctrs.) } \mathrm{mm}\end{array}$ \\
\hline CB060C & 6 & 52 \\
\hline
\end{tabular}

\begin{tabular}{|c|c|c|c|c|c|c|c|}
\hline \multirow{2}{*}{$\begin{array}{l}\text { Unit } \\
\text { No. }\end{array}$} & \multirow{2}{*}{$\begin{array}{c}\text { Concrete } \\
\text { Strength, } \\
\mathrm{MPa}\end{array}$} & \multirow{2}{*}{$\begin{array}{c}\text { Axial } \\
\text { Load } \\
\mathrm{kN}\end{array}$} & \multirow[t]{2}{*}{$\frac{\text { Axial Load }}{\text { Axial Capacity }}$} & \multicolumn{2}{|c|}{$\begin{array}{l}\text { Longitudinal } \\
\text { Reinforcement }\end{array}$} & \multicolumn{2}{|c|}{$\begin{array}{c}\text { Transverse } \\
\text { Reinforcement }\end{array}$} \\
\hline & & & & $\rho^{*}$ & $\begin{array}{c}f_{y} \\
M P a\end{array}$ & $\rho$ & $\begin{array}{c}\mathrm{f}_{\mathrm{y}} \\
\mathrm{MPa}\end{array}$ \\
\hline CB060C & 46.3 & 2632 & 0.74 & $\overline{0.0412}$ & 441 & 0.0089 & 414 \\
\hline
\end{tabular}

Longitudinal steel - twenty-four deformed bars, $13 \mathrm{~mm}$ dia

${ }^{*}$ Calculated from data given in report

Half height of specimen: $\mathrm{L}=323 \mathrm{~mm}$

Data File:

AMCB060C.WK1 


\section{Tests Conducted in North America}

\section{Wight and Sozen}
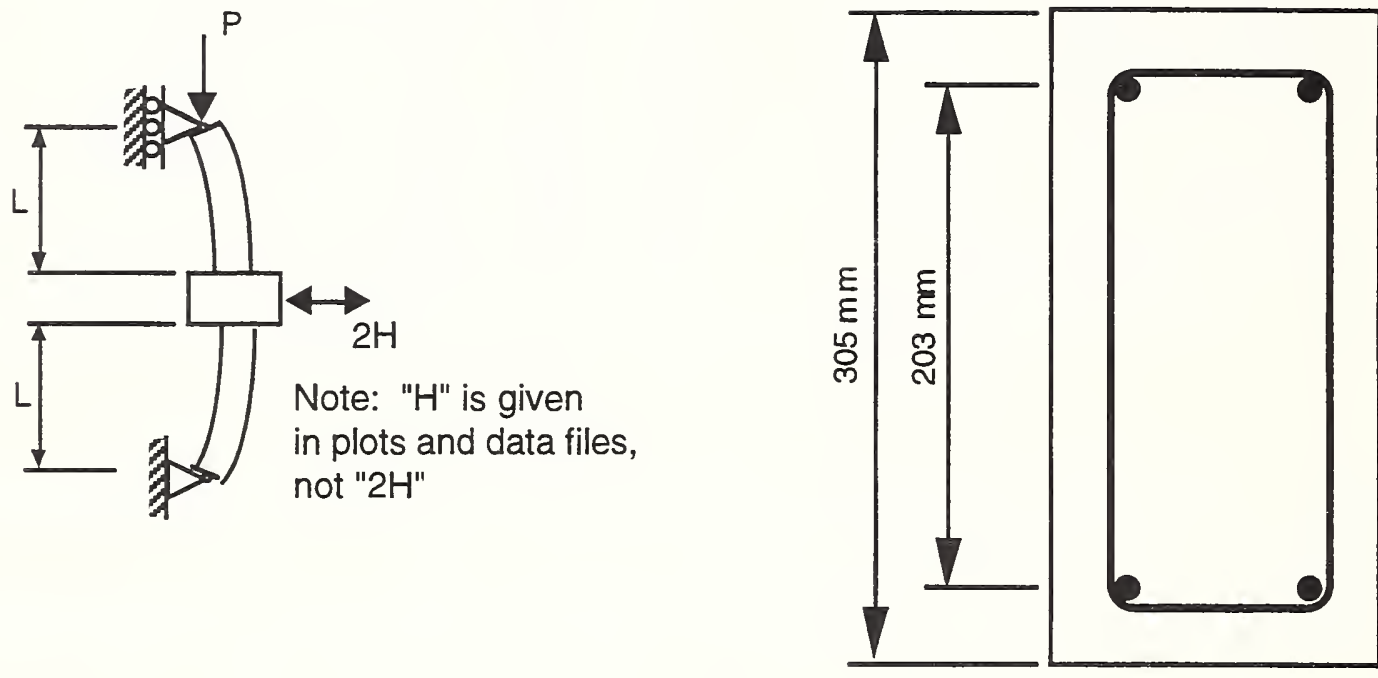

(hoops are welded)

Wight, J.K.; and Sozen, M.A., "Shear Strength Decay in Reinforced Concrete Columns Subjected to Large Deflection Reversals," Structural Research Series No. 403, Civil Engineering Studies, University of Illinois, Urbana-Champaign, Ill., Aug. 1973, 290 pages.

The main variables studied in this series were the axial load level, the transverse reinforcement content, and the displacement ductility demand. Twelve double-ended specimens were tested, but axial load was applied to only seven of the specimens. The central stub of each column was clamped so that it was immobile. Axial loads were applied by a self-reacting system of high strength steel rods and hydraulic ram. Lateral loads were applied by actuators at each end of the specimen; bending was about the strong axis of the cross section. Data was given for each end of the specimen, so in this report 14 load-displacement plots are presented: two plots for each axially loaded specimen.

\begin{tabular}{|c|c|c|}
\hline \multirow{2}{*}{$\begin{array}{l}\text { Unit } \\
\text { No. }\end{array}$} & \multicolumn{2}{|c|}{ Lateral Reinforcement } \\
\hline & $\begin{array}{c}\text { Bar dia. } \\
\text { mm }\end{array}$ & $\begin{array}{l}\text { Spacing } \\
\text { (ctrs.) } \mathrm{mm}\end{array}$ \\
\hline $40.033 a$ & 6.3 & 127 \\
\hline 40.048 & 6.3 & 89 \\
\hline 40.033 & 6.3 & 127 \\
\hline 25.033 & $6 . \overline{3}$ & 127 \\
\hline 40.067 & 6.3 & 64 \\
\hline 40.147 & 9.5 & 64 \\
\hline 40.092 & 9.5 & 102 \\
\hline
\end{tabular}




\begin{tabular}{|c|c|c|c|c|c|c|c|}
\hline \multirow{2}{*}{$\begin{array}{c}\text { Unit } \\
\text { No. }\end{array}$} & $\begin{array}{c}\text { Concrete } \\
\text { Strength, } \\
\text { MPa }\end{array}$ & $\begin{array}{c}\text { Axial } \\
\text { Load } \\
\mathrm{kN}\end{array}$ & \multirow{2}{*}{$\begin{array}{c}\text { Axial Load } \\
\text { Axial Capacity* }\end{array}$} & \multicolumn{2}{|c|}{$\begin{array}{c}\text { Longitudinal } \\
\text { Reinforcement }\end{array}$} & \multicolumn{2}{|c|}{$\begin{array}{c}\text { Transverse } \\
\text { Reinforcement }\end{array}$} \\
\cline { 5 - 8 } & & & & $\rho *$ & $\begin{array}{c}\mathrm{f}_{\mathrm{y}} \\
\mathrm{MPa}\end{array}$ & $\rho$ & $\begin{array}{c}\mathrm{f}_{\mathrm{y}} \\
\mathrm{MPa}\end{array}$ \\
\hline $40.033 \mathrm{a}$ & 34.7 & 189 & 0.12 & 0.0061 & 496 & 0.0033 & 345 \\
\hline 40.048 & 26.1 & 178 & 0.15 & 0.0061 & 496 & 0.0048 & 345 \\
\hline 40.033 & 33.6 & 178 & 0.11 & 0.0061 & 496 & 0.0033 & 345 \\
\hline 25.033 & 33.6 & 111 & 0.07 & 0.0061 & 496 & 0.0033 & 345 \\
\hline 40.067 & 33.4 & 178 & 0.11 & 0.0061 & 496 & 0.0067 & 345 \\
\hline 40.147 & 33.5 & 178 & 0.11 & 0.0061 & 496 & 0.0147 & 317 \\
\hline 40.092 & 35.5 & 178 & 0.11 & 0.0061 & 496 & 0.0092 & 317 \\
\hline
\end{tabular}

Longitudinal steel -4 deformed bars, $19 \mathrm{~mm}$ dia

Lateral reinforcement: $6.3 \mathrm{~mm}$ dia. bars are undeformed; $9.5 \mathrm{~mm}$ dia. bars are deformed

${ }^{*}$ Calculated from data given in report

Half height of specimens: $\mathrm{L}=876 \mathrm{~mm}$

Data Files:

\author{
WS25033E.WK1 \\ WS25033W.WK1 \\ WS033AE.WK1 \\ WS033AW.WK1 \\ WS033E.WK1 \\ WS033W.WK1 \\ WS048E.WK1 \\ WS048W.WK1 \\ WS067E.WK1 \\ WS067W.WK1 \\ WS092E.WK1 \\ WS092W.WK1 \\ WS147E.WK1 \\ WS147W.WK1
}



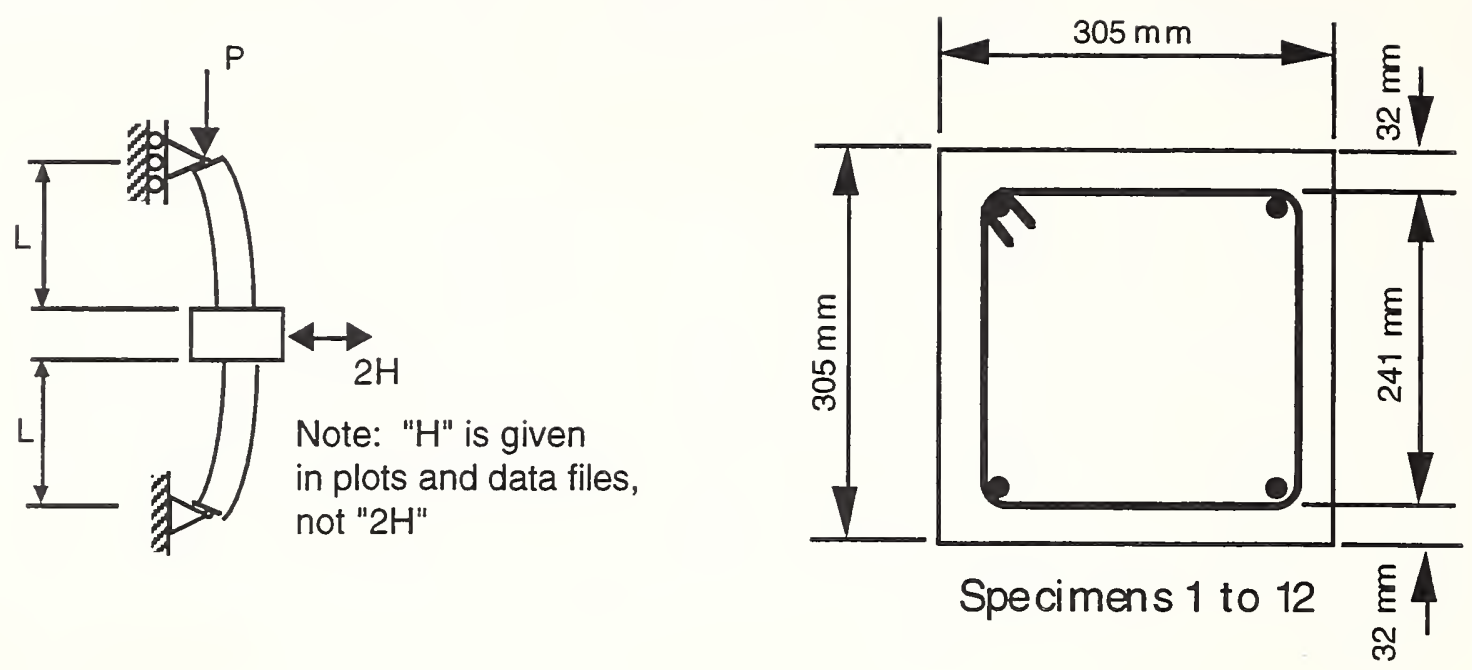

Atalay, M.B.; and Penzien, J., "The Seismic Behaviour of Critical Regions of Reinforced Concrete Components as Influenced by Moment, Shear and Axial Force," Report No. EERC 7519, University of California, Berkeley, Dec. 1975, 226 pages.

The main variables studied in this series were the level of axial stress; the quantity of lateral reinforcement; and the displacement history applied to the specimen. Twelve double-ended specimens were tested, however, only ten of the analog load-deflection plots could be digitized. The two ends of each specimen had pinned boundary condition, and cyclic lateral displacements were applied to the central stub. Axial loads were applied by a hydraulic actuator reacting against a buttress. (Note that a second loading sequence was applied to specimens 1 to 8 , but data from the second loading sequence is not reported here).

\begin{tabular}{|c|c|c|}
\hline \multirow{2}{*}{$\begin{array}{c}\text { Unit } \\
\text { No. }\end{array}$} & \multicolumn{2}{|c|}{ Lateral Reinforcement } \\
\cline { 2 - 3 } & $\begin{array}{c}\text { Bar dia. } \\
\text { mm }\end{array}$ & $\begin{array}{c}\text { Spacing } \\
\text { (ctrs.) mm }\end{array}$ \\
\hline 1S1 & 9.5 & 76 \\
\hline 2 S1 & 9.5 & 127 \\
\hline 3 S1 & 9.5 & 76 \\
\hline 4 S1 & 9.5 & 127 \\
\hline $5 \mathrm{~S} 1$ & 9.5 & 76 \\
\hline $6 \mathrm{~S} 1$ & 9.5 & 127 \\
\hline 9 & 9.5 & 76 \\
\hline 10 & 9.5 & 127 \\
\hline 11 & 9.5 & 76 \\
\hline 12 & 9.5 & 127 \\
\hline
\end{tabular}




\begin{tabular}{|c|c|c|c|c|c|c|c|}
\hline \multirow{2}{*}{$\begin{array}{l}\text { Unit } \\
\text { No. }\end{array}$} & $\begin{array}{c}\text { Concrete } \\
\text { Strength, } \\
\text { MPa }\end{array}$ & $\begin{array}{c}\text { Axial } \\
\text { Load } \\
\mathrm{kN}\end{array}$ & \multirow{2}{*}{$\begin{array}{c}\text { Axial Load } \\
\text { Axial Capacity* }\end{array}$} & \multicolumn{2}{|c|}{$\begin{array}{c}\text { Longitudinal } \\
\text { Reinforcement }\end{array}$} & \multicolumn{2}{|c|}{$\begin{array}{c}\text { Transverse } \\
\text { Reinforcement }\end{array}$} \\
\cline { 5 - 8 } & & & & $\rho *$ & $\begin{array}{c}\mathrm{f}_{\mathrm{y}} \\
\mathrm{MPa}\end{array}$ & $\rho$ & $\begin{array}{c}\mathrm{f}_{\mathrm{y}} \\
\mathrm{MPa}_{\mathrm{y}}\end{array}$ \\
\hline 1S1 & 29.1 & 267 & 0.099 & 0.0167 & 367 & 0.0154 & 363 \\
\hline $2 \mathrm{~S} 1$ & 30.7 & 267 & 0.094 & 0.0167 & 367 & 0.0093 & 363 \\
\hline $3 \mathrm{~S} 1$ & 29.2 & 267 & 0.098 & 0.0167 & 367 & 0.0154 & 363 \\
\hline 4S1 & 27.6 & 267 & 0.104 & 0.0167 & 429 & 0.0093 & 363 \\
\hline $5 \mathrm{~S} 1$ & 29.4 & 534 & 0.196 & 0.0167 & 429 & 0.0154 & 392 \\
\hline $6 \mathrm{~S} 1$ & 31.8 & 534 & 0.181 & 0.0167 & 429 & 0.0093 & 392 \\
\hline 9 & 33.3 & 801 & 0.259 & 0.0167 & 363 & 0.0154 & 392 \\
\hline 10 & 32.4 & 801 & 0.266 & 0.0167 & 363 & 0.0093 & 392 \\
\hline 11 & 31.0 & 801 & 0.278 & 0.0167 & 363 & 0.0154 & 373 \\
\hline 12 & 31.8 & 801 & 0.271 & 0.0167 & 363 & 0.0093 & 373 \\
\hline
\end{tabular}

Longitudinal steel - 4 deformed bars, $22 \mathrm{~mm}$ diameter, grade 40 Lateral reinforcement: $9.5 \mathrm{~mm}$ diameter deformed bars, grade 40

${ }^{*}$ Calculated from data given in report

Half height of specimens: $L=1676 \mathrm{~mm}$

Data Files:

AT75N1S1.WK1
AT75N2S1.WK1
AT75N3S1.WK1
AT75N4S1.WK1
AT75N5S1.WK1
AT75N6S1.WK1
AT75N9.WK1
AT75N10.WK1
AT75N11.WK1
AT75N12.WK1 

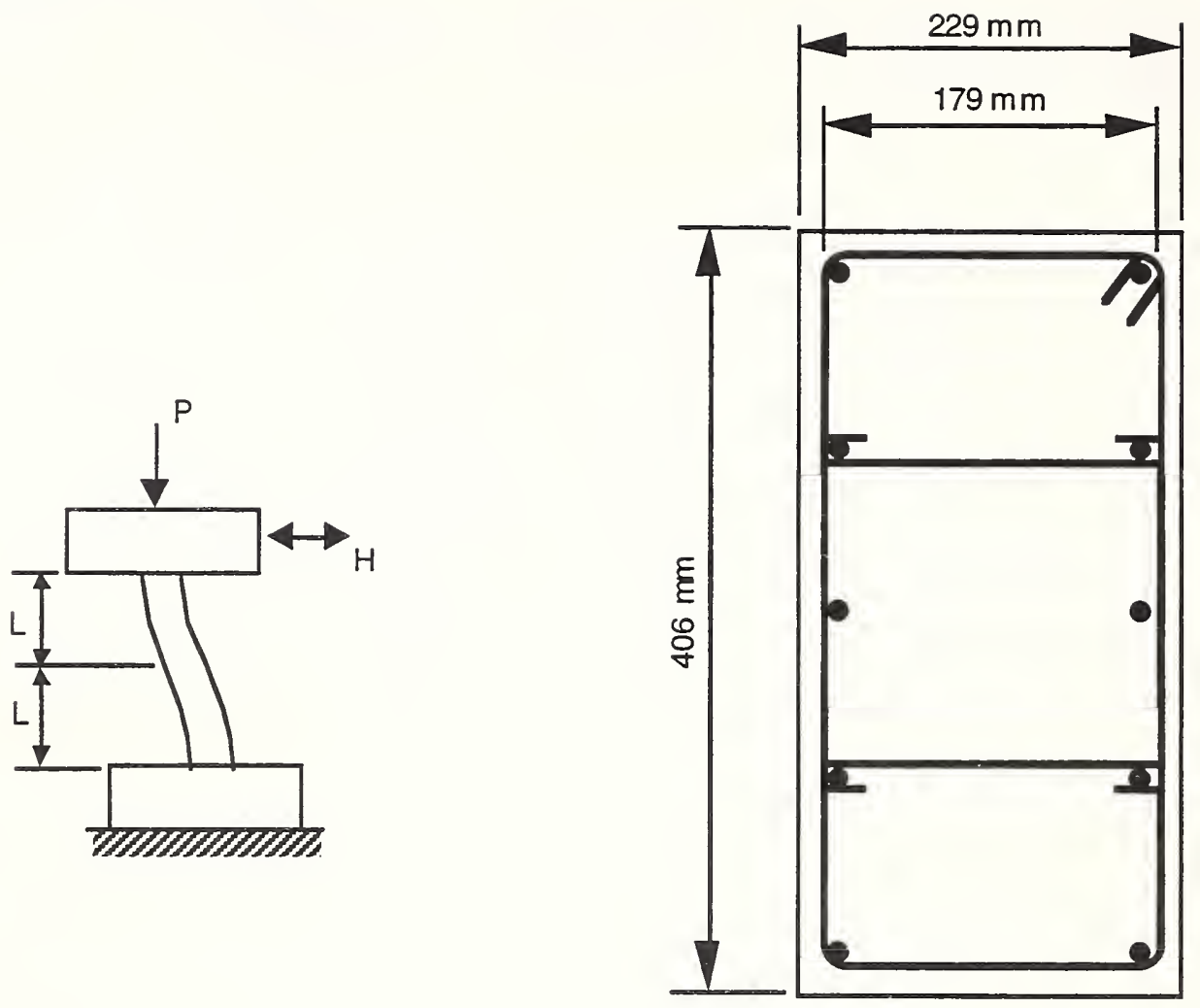

Specimens CUS, CUW, and 2CUS

Umehara, H.; and Jirsa, J.O., "Shear Strength and Deterioration of Short Reinforced Concrete Columns Under Cyclic Deformations," PMFSEL Report No. 82-3, Department of Civil Engineering, University of Texas at Austin, Austin, Texas, July 1982, 256 pages.

Ten short rectangular columns were tested and the results compared to the results of previous tests on square columns. The main variables investigated were the applied lateral load history and the level of axial load. Of the ten columns tested in this series, only three were subjected to both an applied constant axial load and a uniaxial lateral loading path. The ends of the specimens were restrained against rotation.

\begin{tabular}{|c|c|c|c|}
\hline \multirow{2}{*}{$\begin{array}{l}\text { Unit } \\
\text { No. }\end{array}$} & \multirow{2}{*}{$\begin{array}{c}\text { Direction of } \\
\text { Bending }\end{array}$} & & \multicolumn{2}{|c|}{ Lateral Reinforcement } \\
\cline { 3 - 4 } & & $\begin{array}{c}\text { Bar dia. } \\
\mathrm{mm}\end{array}$ & $\begin{array}{c}\text { Spacing } \\
\text { (ctrs.) mm }\end{array}$ \\
\hline CUS & Strong & 6 & 64 \\
\hline CUW & Weak & 6 & 64 \\
\hline 2CUS & Strong. & 6 & 64 \\
\hline
\end{tabular}




\begin{tabular}{|c|c|c|c|c|c|c|c|}
\hline \multirow{2}{*}{$\begin{array}{c}\text { Unit } \\
\text { No. }\end{array}$} & $\begin{array}{c}\text { Concrete } \\
\text { Strength, } \\
\text { MPa }\end{array}$ & $\begin{array}{c}\text { Axial } \\
\text { Load } \\
\mathrm{kN}\end{array}$ & \multirow{2}{*}{$\begin{array}{c}\text { Axial Load } \\
\text { Axial Capacity* }\end{array}$} & \multicolumn{2}{|c|}{$\begin{array}{c}\text { Longitudinal } \\
\text { Reinforcement }\end{array}$} & \multicolumn{2}{|c|}{$\begin{array}{c}\text { Transverse } \\
\text { Reinforcement }\end{array}$} \\
\cline { 5 - 8 } & & & & $\rho *$ & $\begin{array}{c}\mathrm{f}_{\mathrm{y}} \\
\mathrm{MPa}\end{array}$ & $\rho *$ & $\begin{array}{c}\mathrm{f}_{\mathrm{y}} \\
\mathrm{MPa}\end{array}$ \\
\hline CUS & 34.9 & 534 & 0.165 & 0.0306 & 441 & 0.0028 & 414 \\
\hline CUW & 34.9 & 534 & 0.165 & 0.0306 & 441 & 0.0031 & 414 \\
\hline 2CUS & 42.0 & 1068 & 0.274 & 0.0306 & 441 & 0.0028 & 414 \\
\hline
\end{tabular}

Longitudinal steel - 10 deformed bars, $19 \mathrm{~mm}$ diameter

Lateral reinforcement: $6 \mathrm{~mm}$ diameter deformed bars

${ }^{*}$ Calculated from data given in report

Half height of specimens: $L=457 \mathrm{~mm}$

Data Files: $\quad$ UMECUS.WK1

UMECUW.WK1

UME2CUS.WK1 

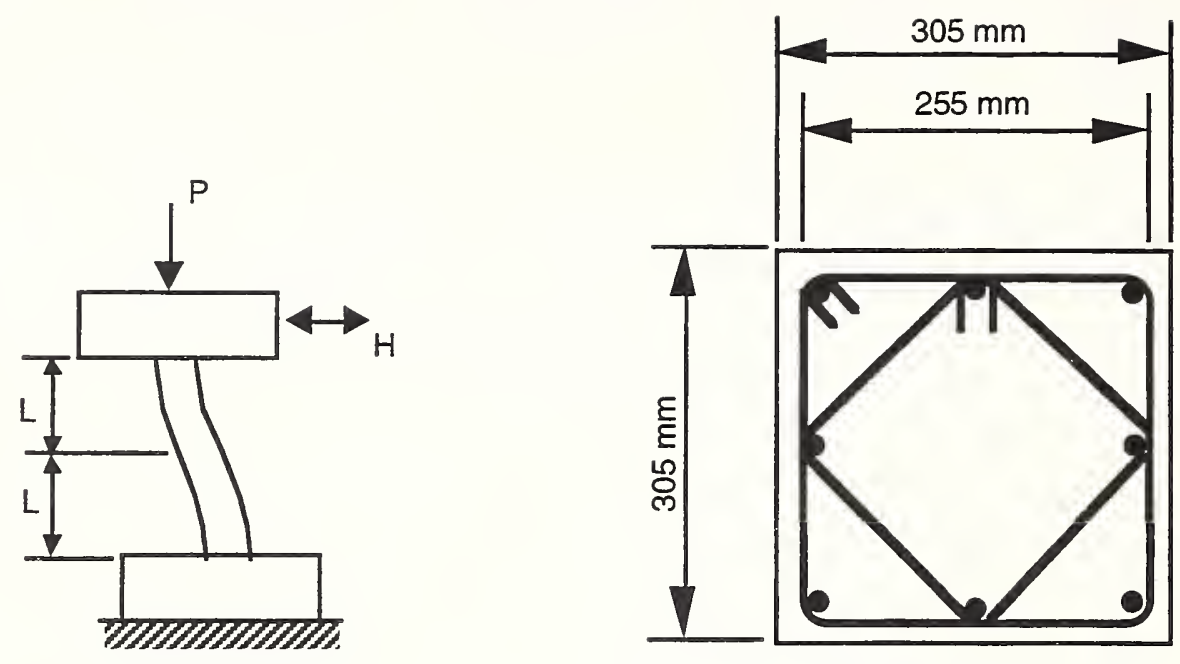

Specimen 11

Bett, Bart J.; Klingner, Richard E.; and Jirsa, James O., "Behavior of Strengthened and Repaired Reinforced Concrete Columns Under Cyclic Deformations," PMFSEL Report No. 85-3, Department of Civil Engineering, University of Texas at Austin, Austin, Texas, December 1985, 75 pages.

Three columns at two-thirds scale were tested. Two columns were strengthened by adding reinforced concrete jackets before testing. Results from these strengthened specimens are not reported here; only data from the one unstrengthened specimen were digitized for this report. The specimens were subjected to a constant axial load, and the ends of the specimens were restrained against rotation.

\begin{tabular}{|c|c|c|}
\hline \multirow{2}{*}{$\begin{array}{l}\text { Unit } \\
\text { No. }\end{array}$} & \multicolumn{2}{|c|}{ Lateral Reinforcement } \\
\hline & $\begin{array}{c}\text { Bar dia. } \\
\text { mm }\end{array}$ & $\begin{array}{l}\text { Spacing } \\
\text { (ctrs.) } \mathrm{mm}\end{array}$ \\
\hline $1-1$ & 6 & 203 \\
\hline
\end{tabular}

\begin{tabular}{|c|c|c|c|c|c|c|c|}
\hline $\begin{array}{c}\text { Unit } \\
\text { No. }\end{array}$ & $\begin{array}{c}\text { Concrete } \\
\text { Strength, } \\
\mathrm{MPa}\end{array}$ & $\begin{array}{c}\text { Axial } \\
\text { Load } \\
\mathrm{kN}\end{array}$ & \multicolumn{2}{|c|}{$\begin{array}{c}\text { Axial Load } \\
\text { Axial Capacity }\end{array}$} & \multicolumn{2}{|c|}{$\begin{array}{c}\text { Longitudinal } \\
\text { Reinforcement }\end{array}$} & \multicolumn{2}{|c|}{$\begin{array}{c}\text { Transverse } \\
\text { Reinforcement }\end{array}$} \\
\cline { 5 - 8 } & & & $\rho *$ & $\begin{array}{c}\mathrm{f}_{\mathrm{y}} \\
\mathrm{MPa}\end{array}$ & $\rho *$ & $\begin{array}{c}\mathrm{f}_{\mathrm{y}} \\
\mathrm{MPa}\end{array}$ \\
\hline $1-1$ & 29.9 & 288 & 0.104 & 0.0244 & 462 & 0.0026 & 414 \\
\hline
\end{tabular}

Longitudinal steel - eight deformed bars, $19 \mathrm{~mm}$ dia

Lateral reinforcement: $6 \mathrm{~mm}$ diameter deformed bars

${ }^{*}$ Calculated from data given in report

Half height of specimen: $\mathrm{L}=457 \mathrm{~mm}$

Data File:

BETTNO11.WK1 

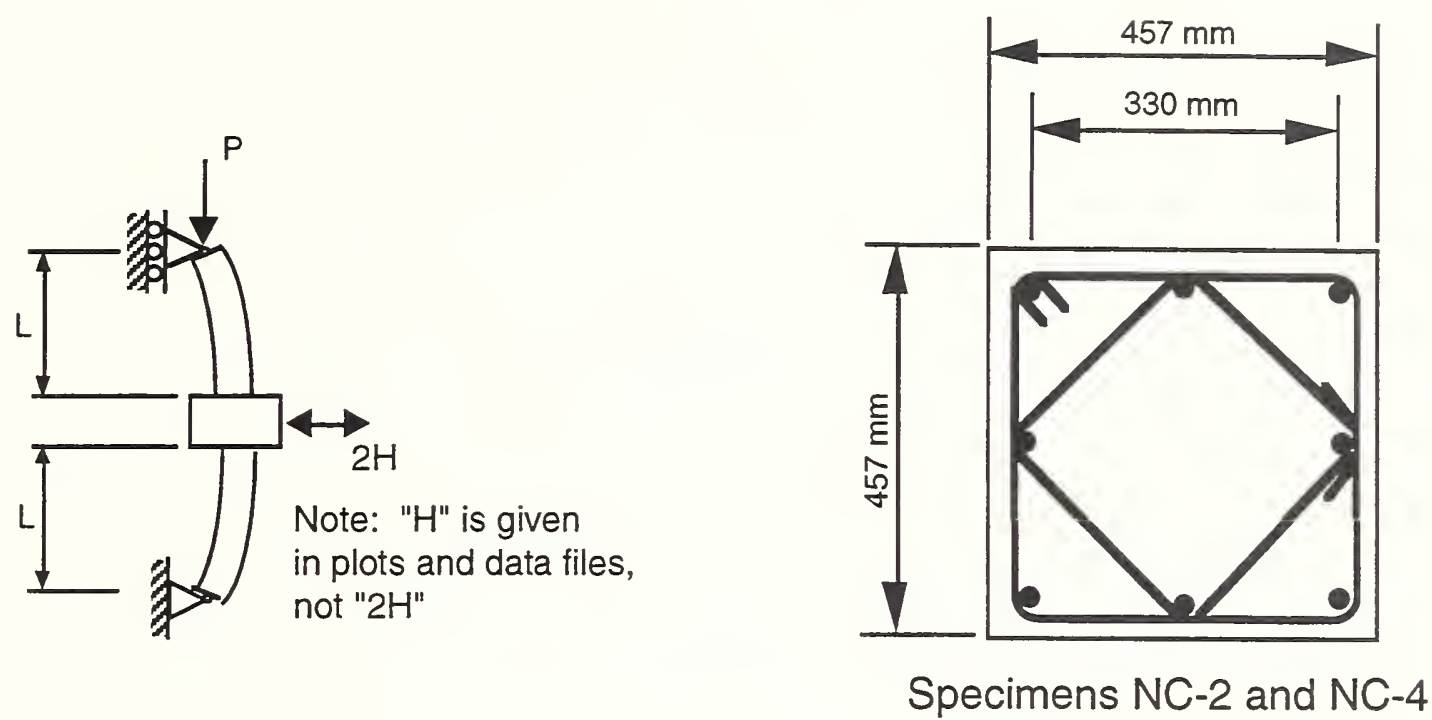

Azizinamini, Atorod; Johal, Lakhpal S.; Hanson, Norman W.; Musser, Donald W.; and Corley, William G., "Effects of Transverse Reinforcement on Seismic Performance of Columns A Partial Parametric Investigation," Project No. CR-9617, Construction Technology Laboratories, Skokie, Illinois, Sept. 1988.

Twelve full-scale tests were conducted. Eleven of the specimens had a square cross section, and one had a circular cross section. The main variables studied were the level of axial load, type of transverse reinforcement, and the amount and spacing of transverse reinforcement. Digitizable load deflection plots were available for only two of the tests on columns with square cross sections. The tests were conducted using a double-ended specimen, a self-reacting load frame with a hydraulic ram to apply cyclic lateral load, and a universal testing machine to apply constant axial load.

\begin{tabular}{|c|c|c|}
\hline \multirow{2}{*}{$\begin{array}{l}\text { Unit } \\
\text { No. }\end{array}$} & \multicolumn{2}{|c|}{ Lateral Reinforcement } \\
\hline & $\begin{array}{c}\text { Bar dia. } \\
\text { mm }\end{array}$ & $\begin{array}{l}\text { Spacing } \\
\text { (ctrs.) } \mathrm{mm}\end{array}$ \\
\hline $\mathrm{NC}-2$ & 12.7 & 102 \\
\hline $\mathrm{NC}-4$ & 9.5 & 102 \\
\hline
\end{tabular}

\begin{tabular}{|c|c|c|c|c|c|c|c|}
\hline $\begin{array}{l}\text { Unit } \\
\text { No. }\end{array}$ & $\begin{array}{c}\text { Concrete } \\
\text { Strength, } \\
\mathrm{MPa}\end{array}$ & $\begin{array}{c}\text { Axial } \\
\text { Load } \\
\mathrm{kN}\end{array}$ & \multicolumn{2}{c|}{$\begin{array}{c}\text { Axial Load } \\
\text { Axial Capacity* }\end{array}$} & \multicolumn{2}{|c|}{$\begin{array}{c}\text { Longitudinal } \\
\text { Reinforcement }\end{array}$} & \multicolumn{2}{|c|}{$\begin{array}{c}\text { Transverse } \\
\text { Reinforcement }\end{array}$} \\
\cline { 4 - 7 } & & & & $\rho$ & $\begin{array}{c}\mathrm{f}_{\mathrm{y}} \\
\mathrm{MPa}\end{array}$ & $\rho$ & $\begin{array}{c}\mathrm{f}_{\mathrm{y}} \\
\mathrm{MPa}\end{array}$ \\
\hline NC-2 & 39.3 & 1690 & 0.206 & 0.0195 & 439 & 0.0219 & 454 \\
\hline NC-4 & 39.8 & 2580 & 0.310 & 0.0195 & 439 & 0.0126 & 616 \\
\hline
\end{tabular}

Longitudinal steel - eight deformed bars, $25.4 \mathrm{~mm}$ diameter

Lateral reinforcement: 12.7 or $9.5 \mathrm{~mm}$ diameter deformed bars

* Calculated from data given in report

Half height of specimen: $\mathrm{L}=1372 \mathrm{~mm}$

Data Files: 


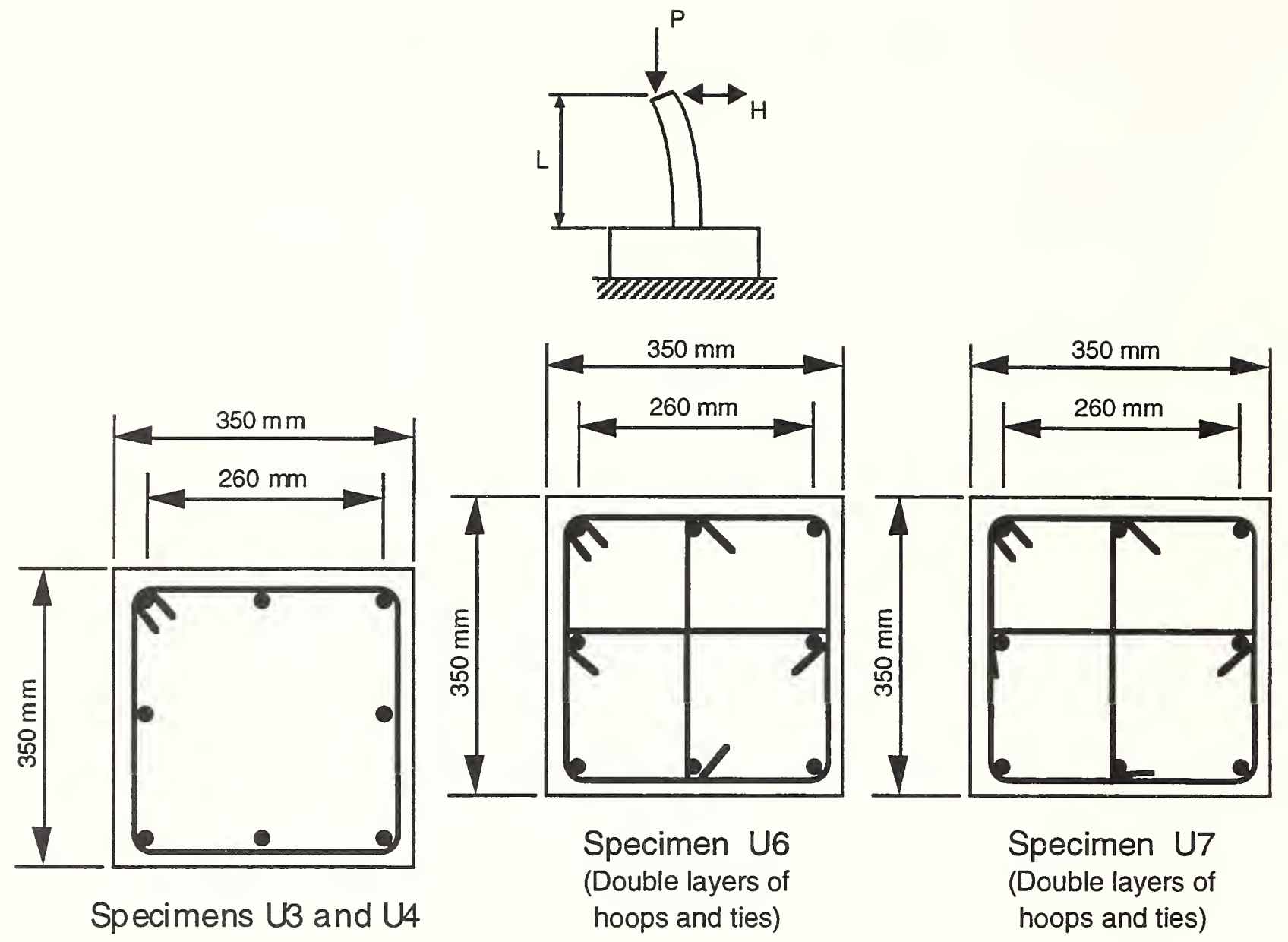

Saatcioglu, Murat; and Ozcebe, Guney, "Response of Reinforced Concrete Columns to Simulated Seismic Loading," American Concrete Institute, ACI Structural Journal, JanuaryFebruary, 1989, pp. 3-12.

The authors conducted a series of tests on 14 cantilever column specimens. The columns cross section was square, and three different lateral reinforcement configurations were tested. The main variables investigated were axial load, confining reinforcement, and deformation path. Of the fourteen specimens, seven had lateral displacements applied along a principal axis of the cross section ("U" series); four had lateral displacements applied along a diagonal of the cross section ("D" series); and two had bidirectional lateral displacement patterns ("B" series). Only certain specimens from the " $U$ " series are presented here. Of the " $U$ " series specimens, $U 1$ had no axial load applied, and U5 had variable axial load, so results for U1 and U5 are not presented. Lateral load vs. lateral displacement data files were obtained directly from the researchers who performed the tests. The data file for specimen U2 was not readable, so data for U2 is not presented. 


\begin{tabular}{|c|c|c|}
\hline \multirow{2}{*}{$\begin{array}{l}\text { Unit } \\
\text { No. }\end{array}$} & \multicolumn{2}{|c|}{ Lateral Reinforcement } \\
\hline & $\begin{array}{c}\text { Bar dia. } \\
\text { mm }\end{array}$ & $\begin{array}{l}\text { Spacing } \\
\text { (ctrs.) } \mathrm{mm}\end{array}$ \\
\hline U3 & 10 & 75 \\
\hline$\overline{\mathrm{U} 4}$ & 10 & 50 \\
\hline$\overline{\text { U6 }}$ & 6.4 & 65 \\
\hline$\overline{\mathrm{U7}}$ & 6.4 & 65 \\
\hline
\end{tabular}

\begin{tabular}{|c|c|c|c|c|c|c|c|}
\hline \multirow{2}{*}{$\begin{array}{l}\text { Unit } \\
\text { No. }\end{array}$} & \multirow{2}{*}{$\begin{array}{c}\text { Concrete } \\
\text { Strength, } \\
\mathrm{MPa}\end{array}$} & \multirow{2}{*}{$\begin{array}{c}\text { Axial } \\
\text { Load } \\
\mathrm{kN}\end{array}$} & \multirow[t]{2}{*}{$\frac{\text { Axial Load }}{\text { Axial Capacity* }}$} & \multicolumn{2}{|c|}{$\begin{array}{l}\text { Longitudinal } \\
\text { Reinforcement }\end{array}$} & \multicolumn{2}{|c|}{$\begin{array}{c}\text { Transverse } \\
\text { Reinforcemen }\end{array}$} \\
\hline & & & & $\rho$ & $\begin{array}{c}f_{y} \\
M P a\end{array}$ & $\rho$ & $\begin{array}{l}\mathrm{f}_{\mathrm{y}} \\
\mathrm{MPa}\end{array}$ \\
\hline U3 & 34.8 & 600 & 0.141 & 0.0327 & 430 & 1.69 & 470 \\
\hline U4 & 32.0 & 600 & 0.153 & 0.0327 & 438 & 2.54 & 470 \\
\hline U6 & 37.3 & 600 & 0.131 & 0.0327 & 437 & 1.95 & 425 \\
\hline U7 & 39.0 & 600 & 0.126 & 0.0327 & 437 & 1.95 & 425 \\
\hline
\end{tabular}

Longitudinal steel - 8 deformed bars, $25 \mathrm{~mm}$ dia

${ }^{*}$ Calculated from data given in report

Height of specimens: $\mathrm{L}=1000 \mathrm{~mm}$

Data Files:

SAATU3.WK1

SAATU4.WK1

SAATU6.WK1

SAATU7.WK1 
$-40-$ 


\section{Chapter 3: Plots of Digitized Lateral Load-Lateral Deflection Data}

This chapter contains plots of digital load-deflection test data for the 93 specimens described in this report. Each plot is identified by the test name shown in the upper left corner of the figure. Note that for double-ended specimens (Figure 2, page 3), the force shown in the plot is one-half the force applied at the center stub of the specimen; and for double curvature specimens (Figure 3 , page 3 ) the displacement shown in the plot is one-half the relative displacement between the two end beams of the specimen. 


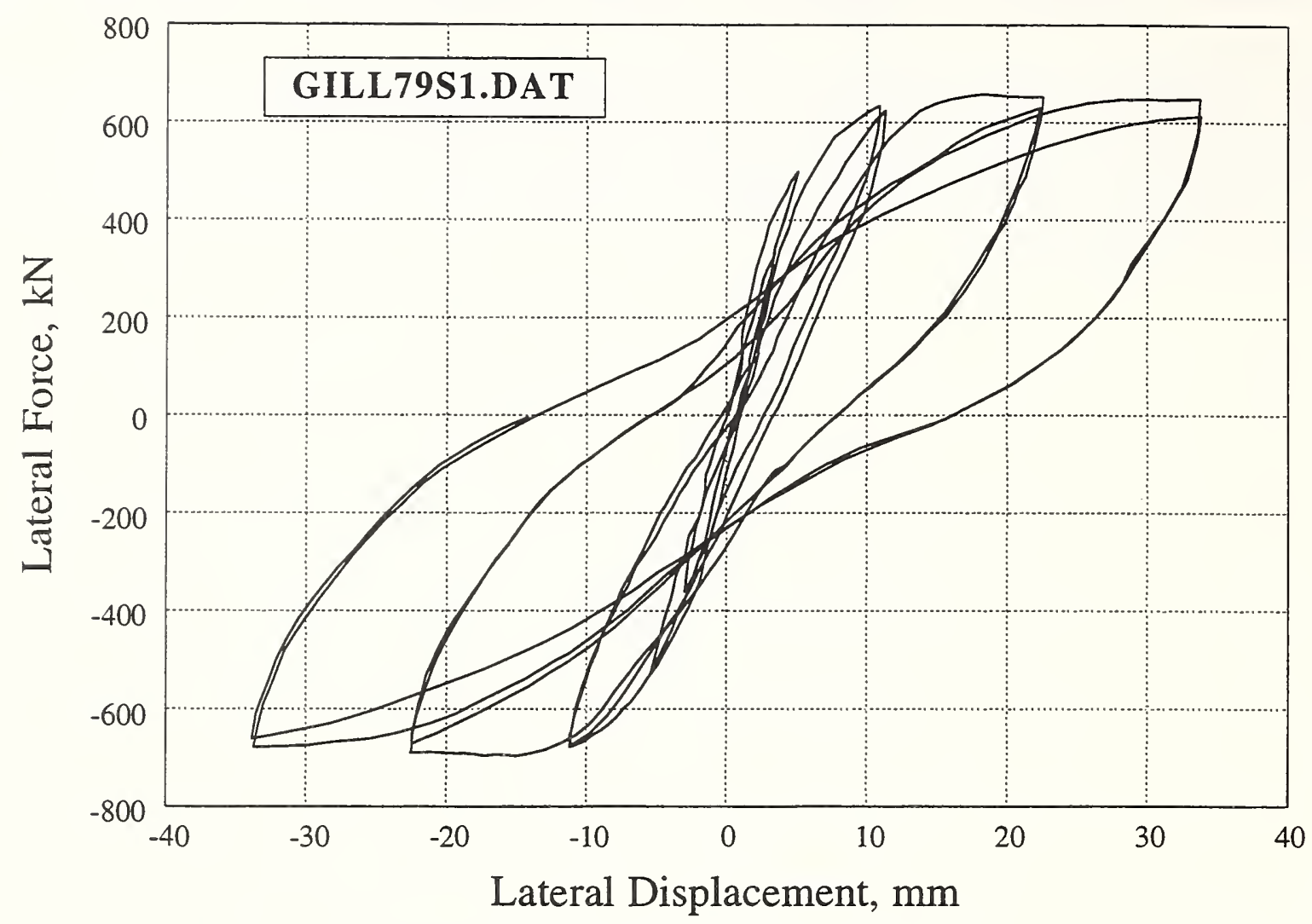

Figure 1. Specimen 1 of Gill 1979

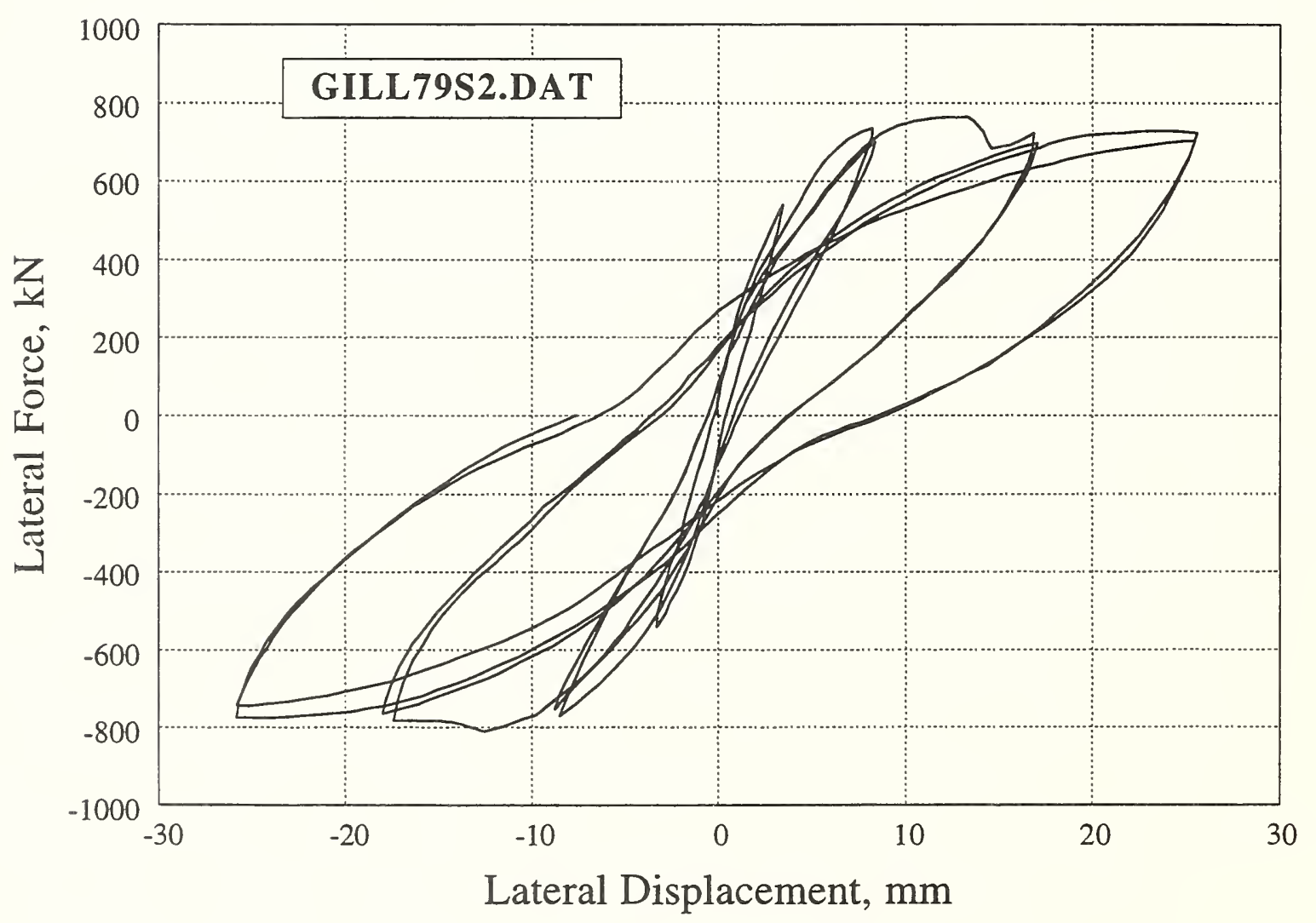

Figure 2. Specimen 2 of Gill 1979 


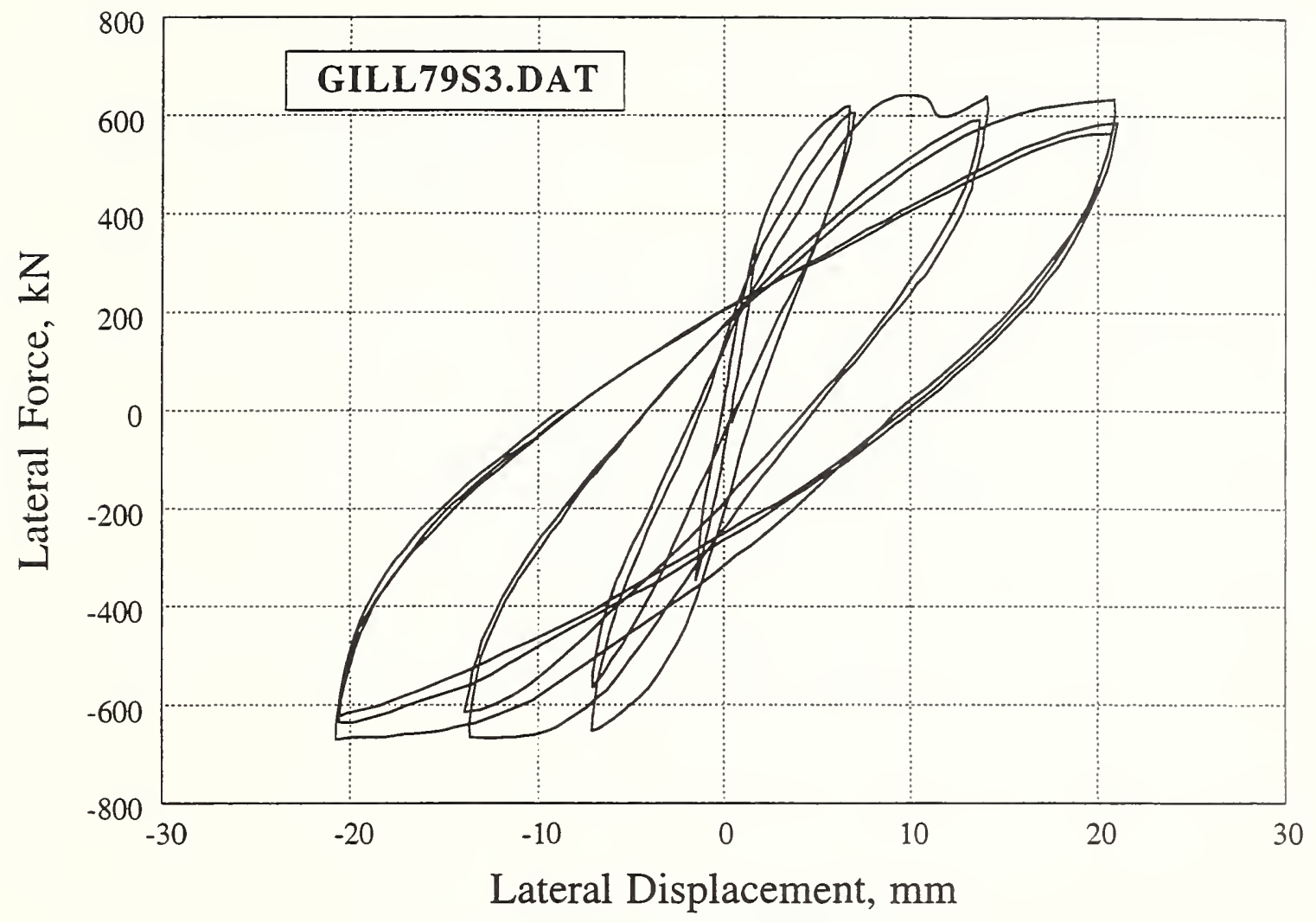

Figure 3. Specimen 3 of Gill 1979

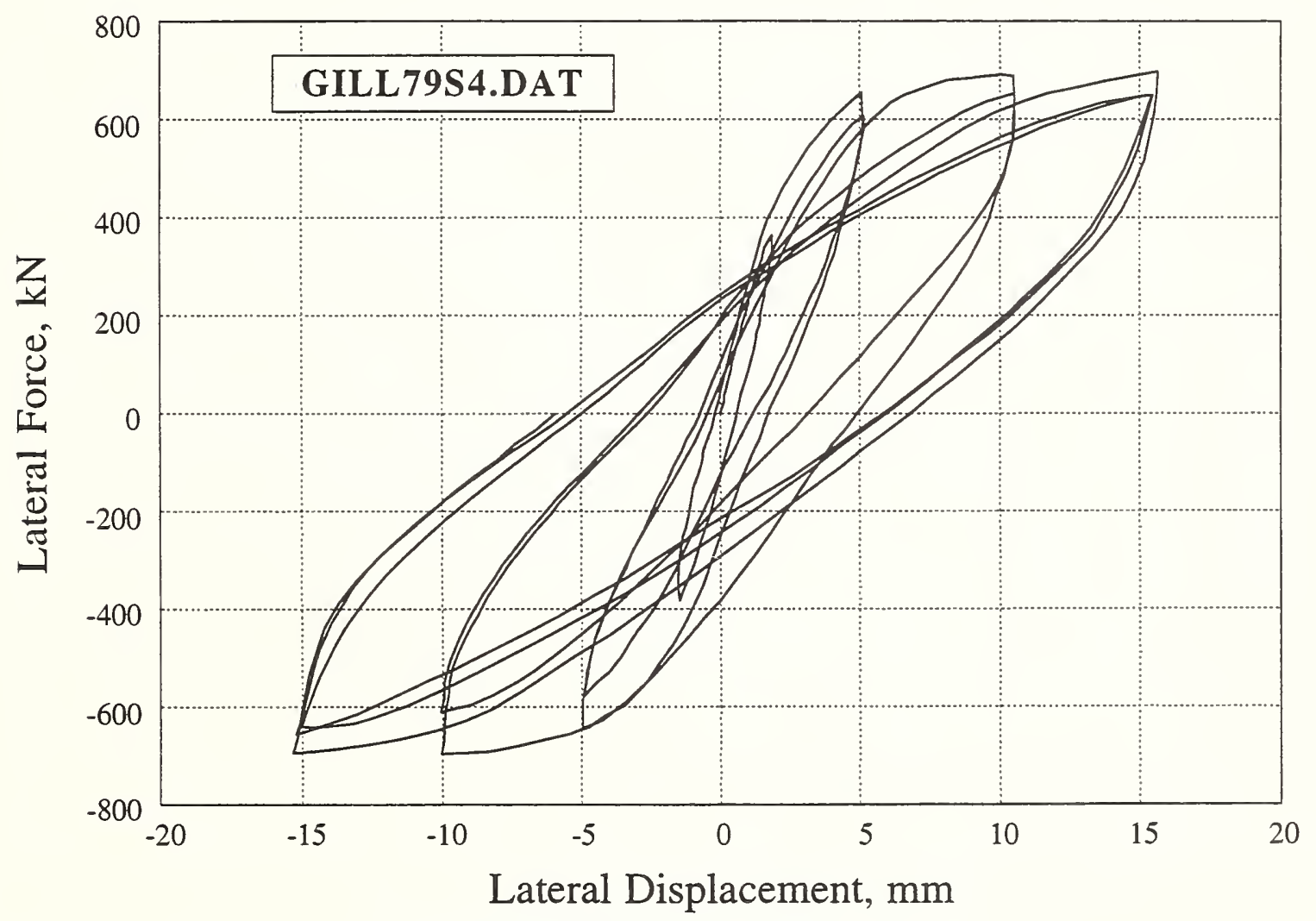

Figure 4. Specimen 4 of Gill 1979 


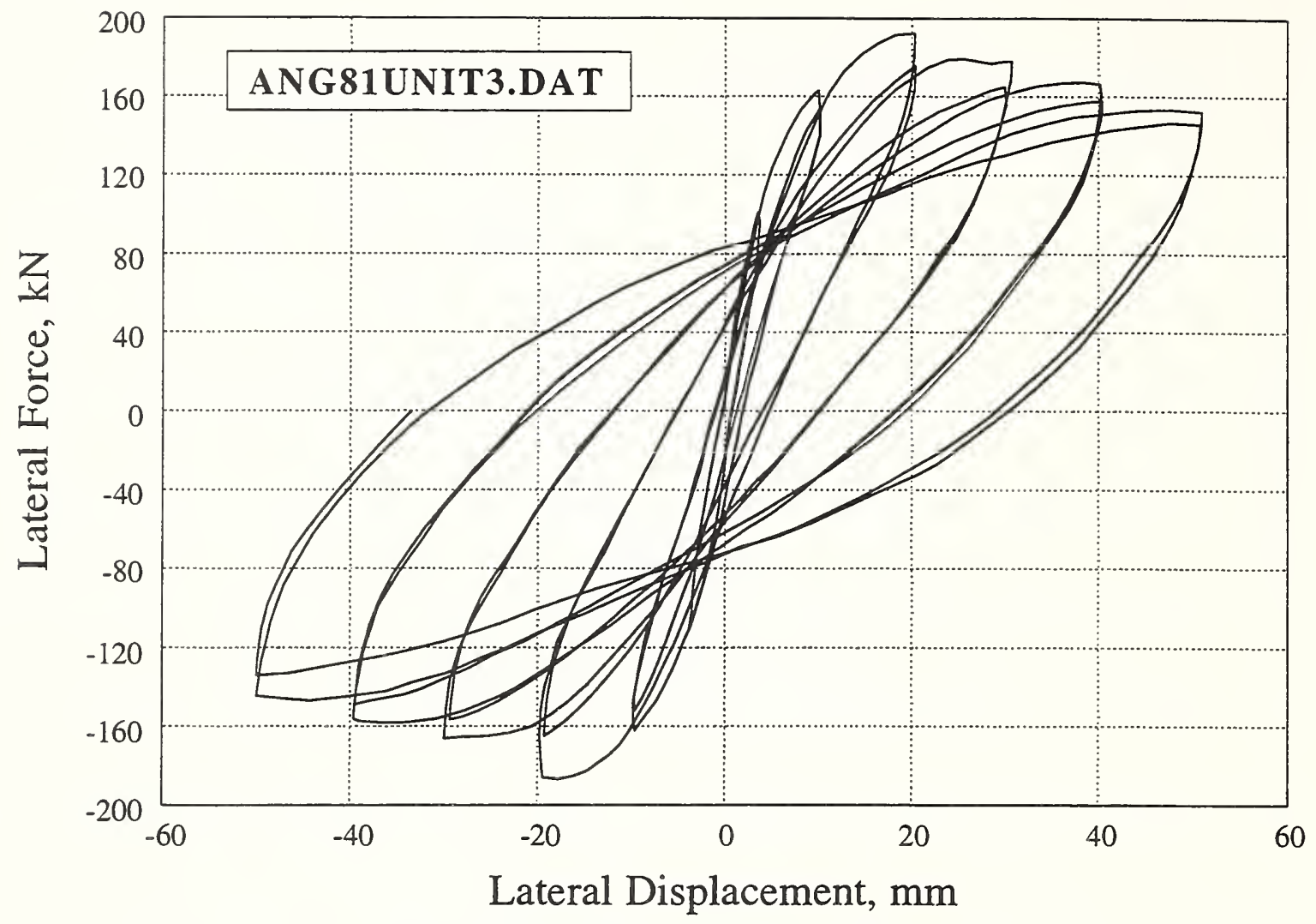

Figure 5. Specimen 3 of Ang 1981

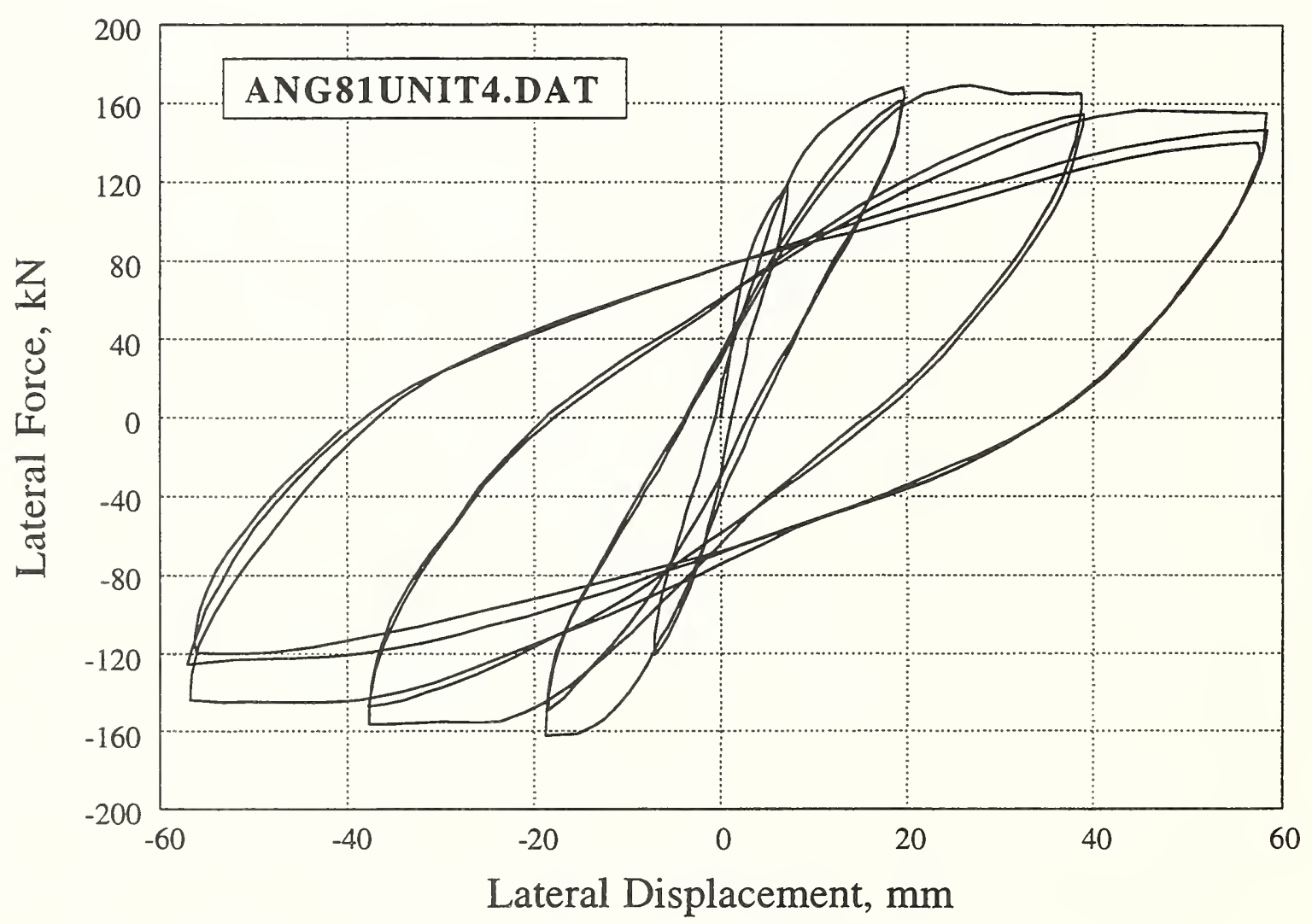

Figure 6. Specimen 4 of Ang 1981 


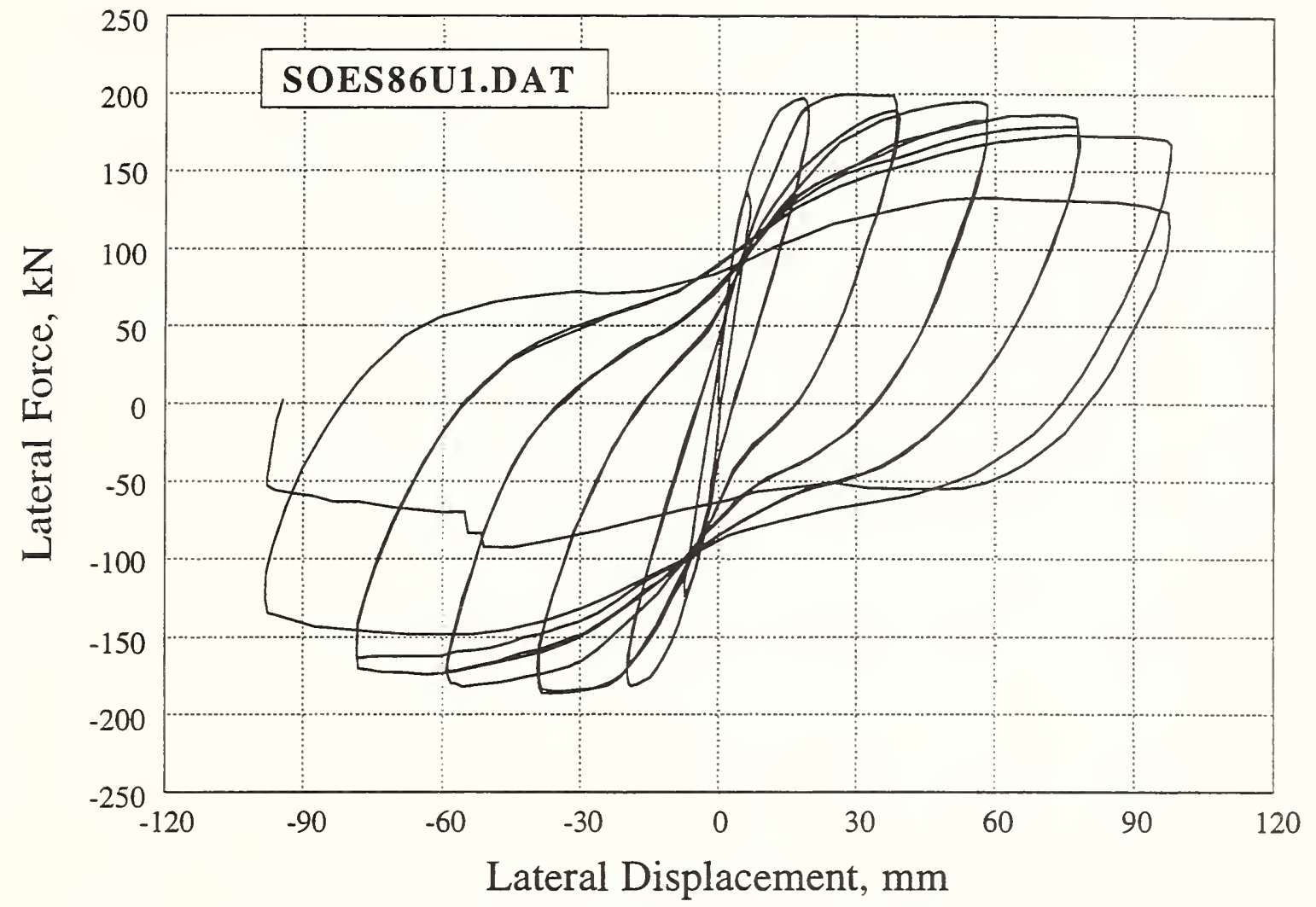

Figure 7. Specimen 1 of Soesianawati 1986

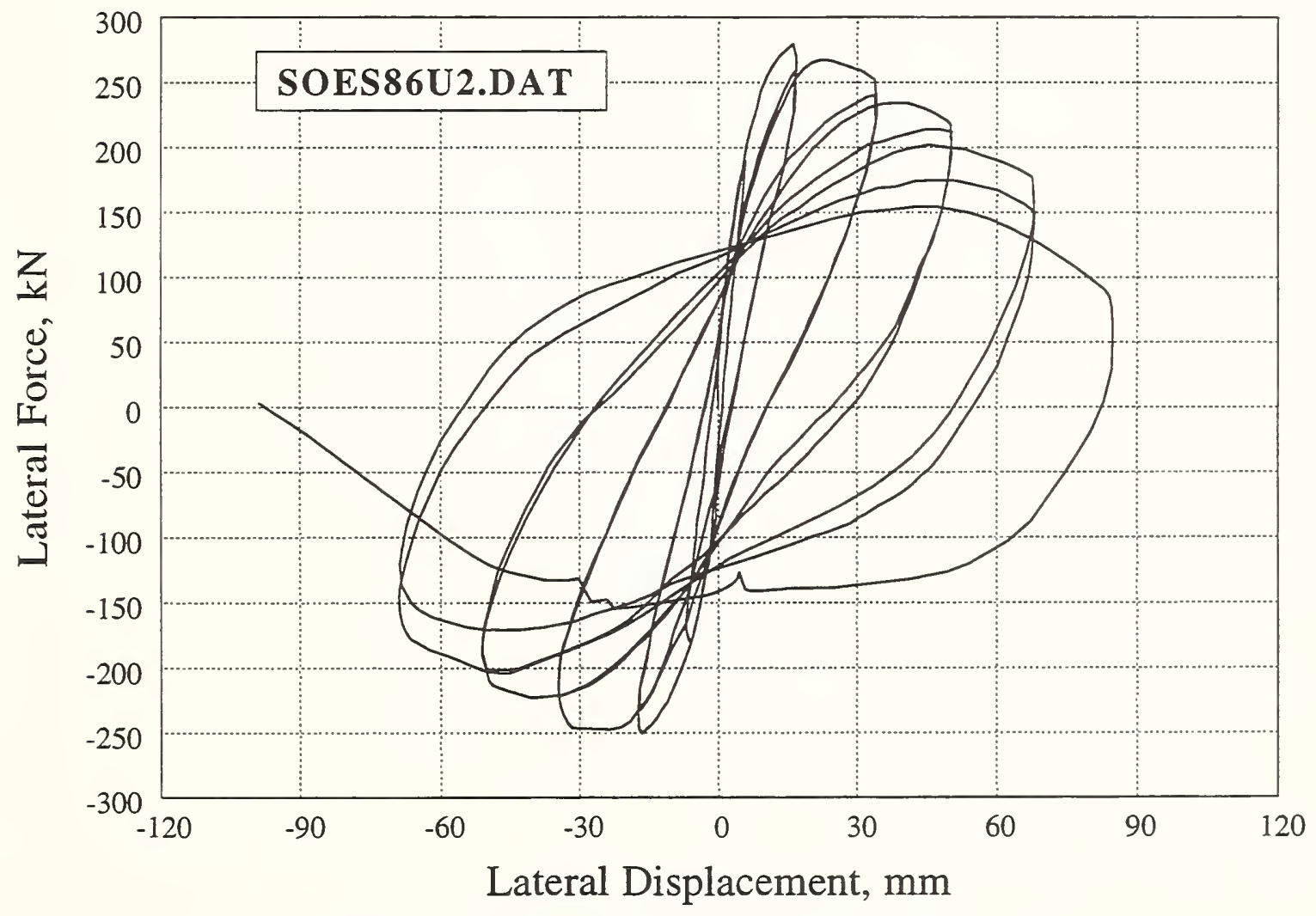

Figure 8. Specimen 2 of Soesianawati 1986 


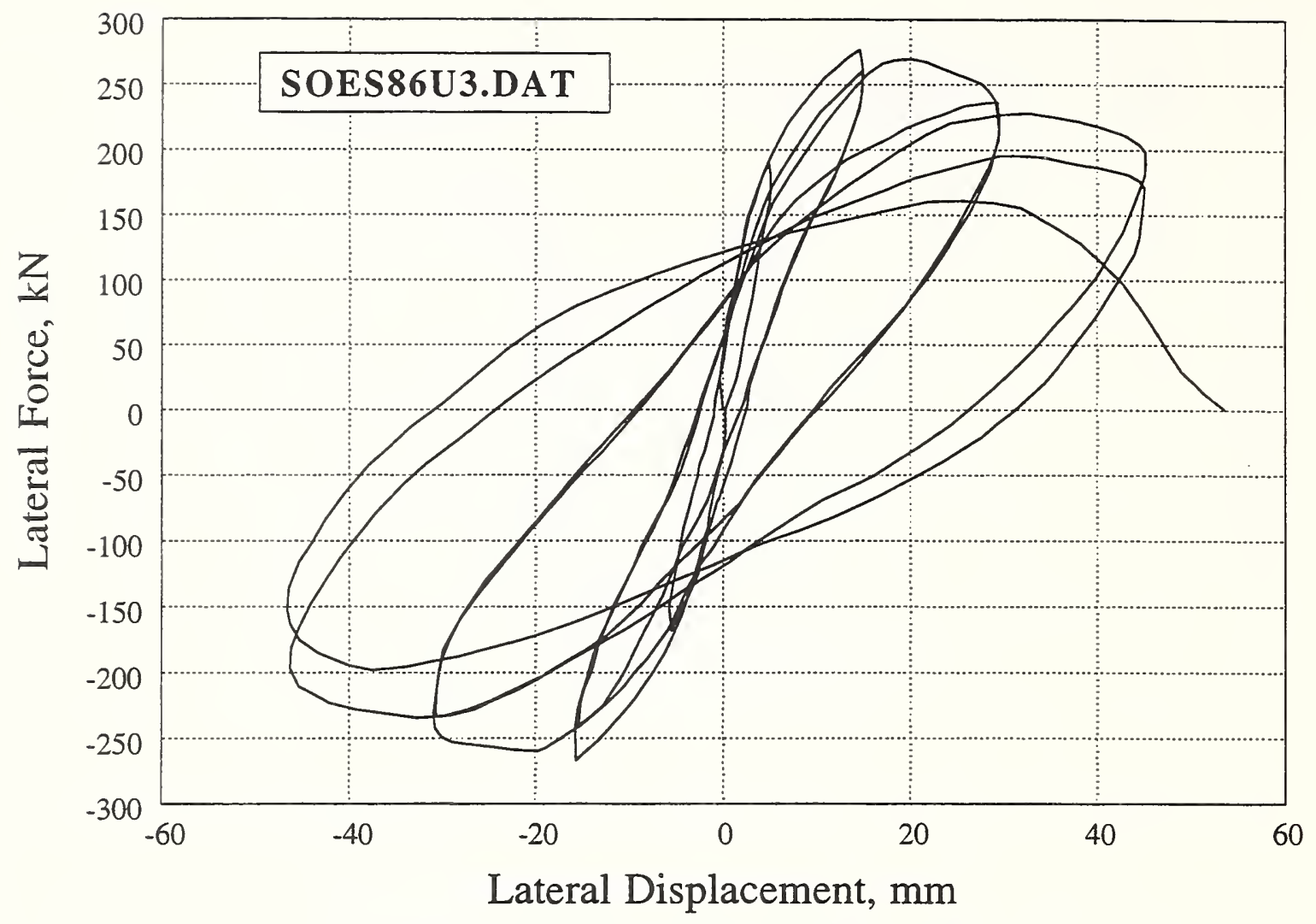

Figure 9. Specimen 3 of Soesianawati 1986

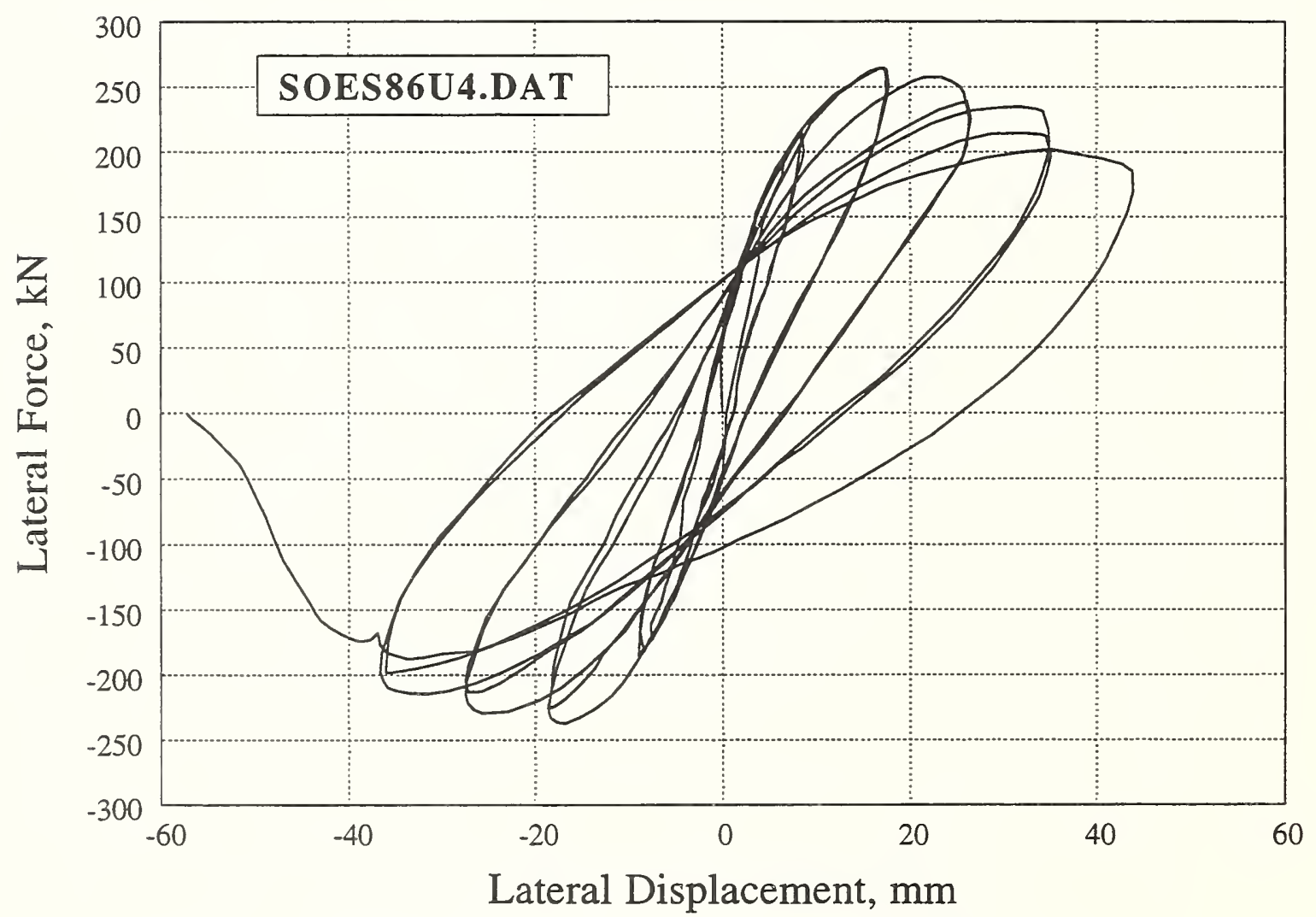

Figure 10. Specimen 4 of Soesianawati 1986 


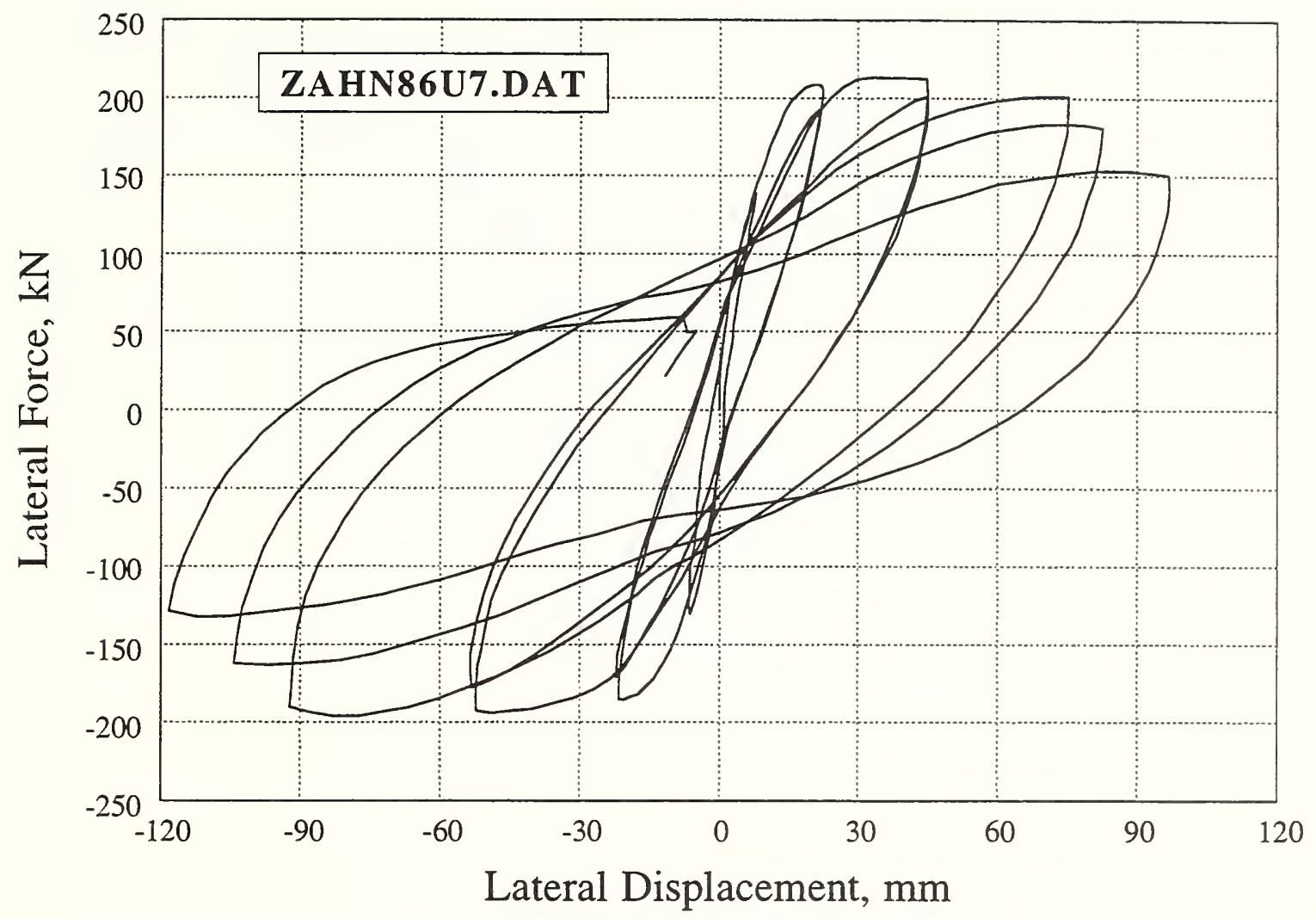

Figure 11. Specimen 7 of Zahn 1986

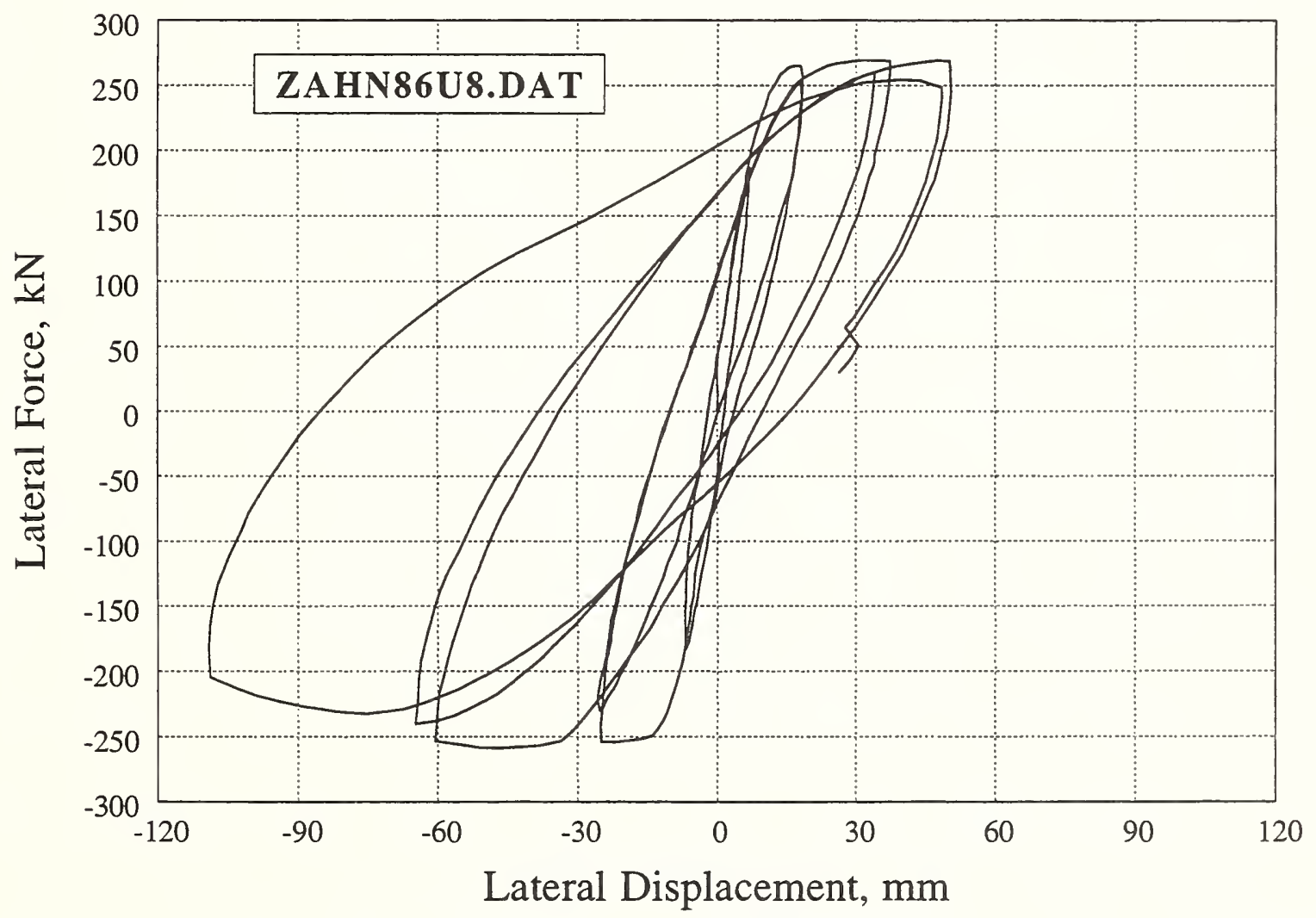

Figure 12. Specimen 8 of Zahn 1986 


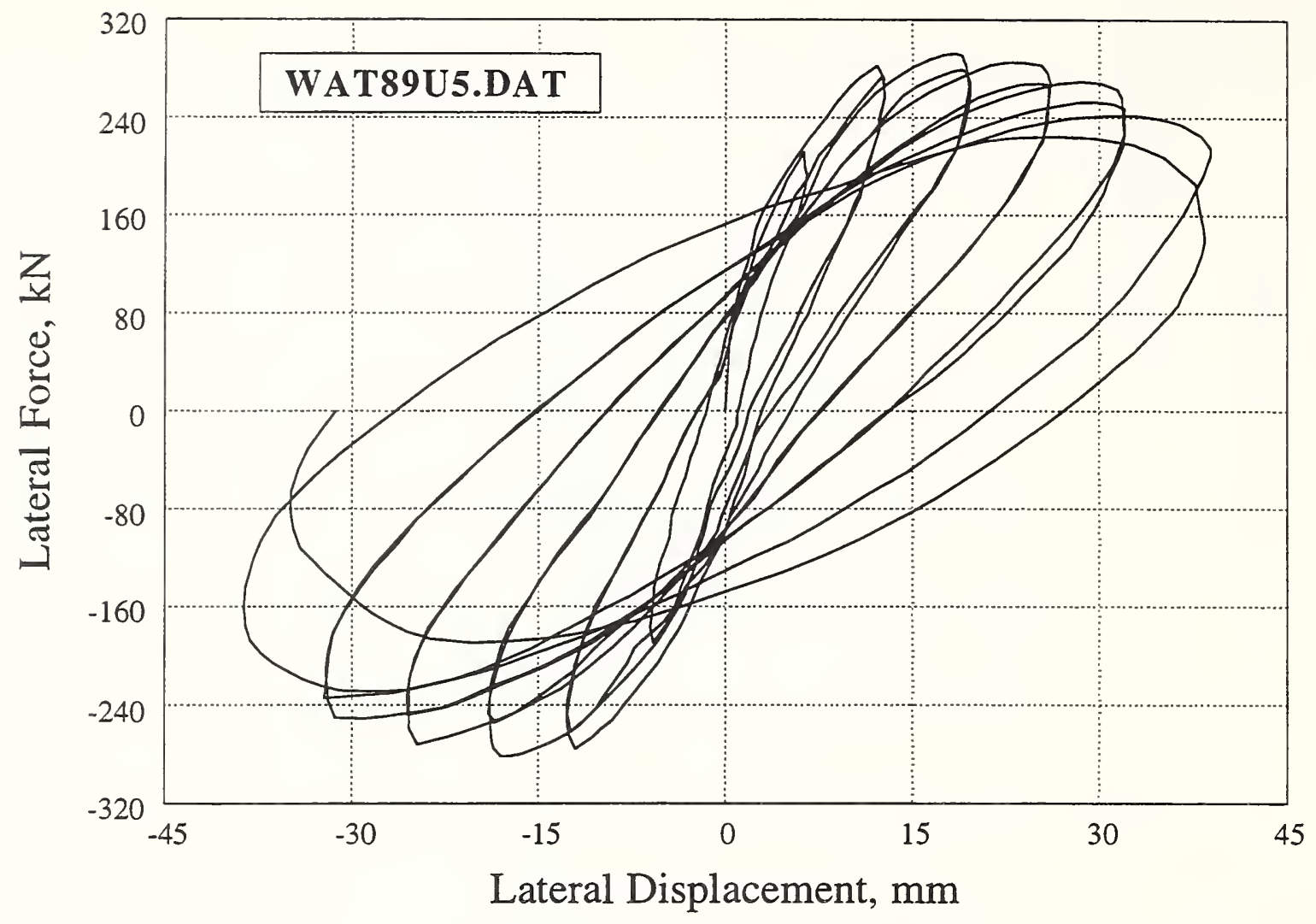

Figure 13. Specimen 5 of Watson 1989

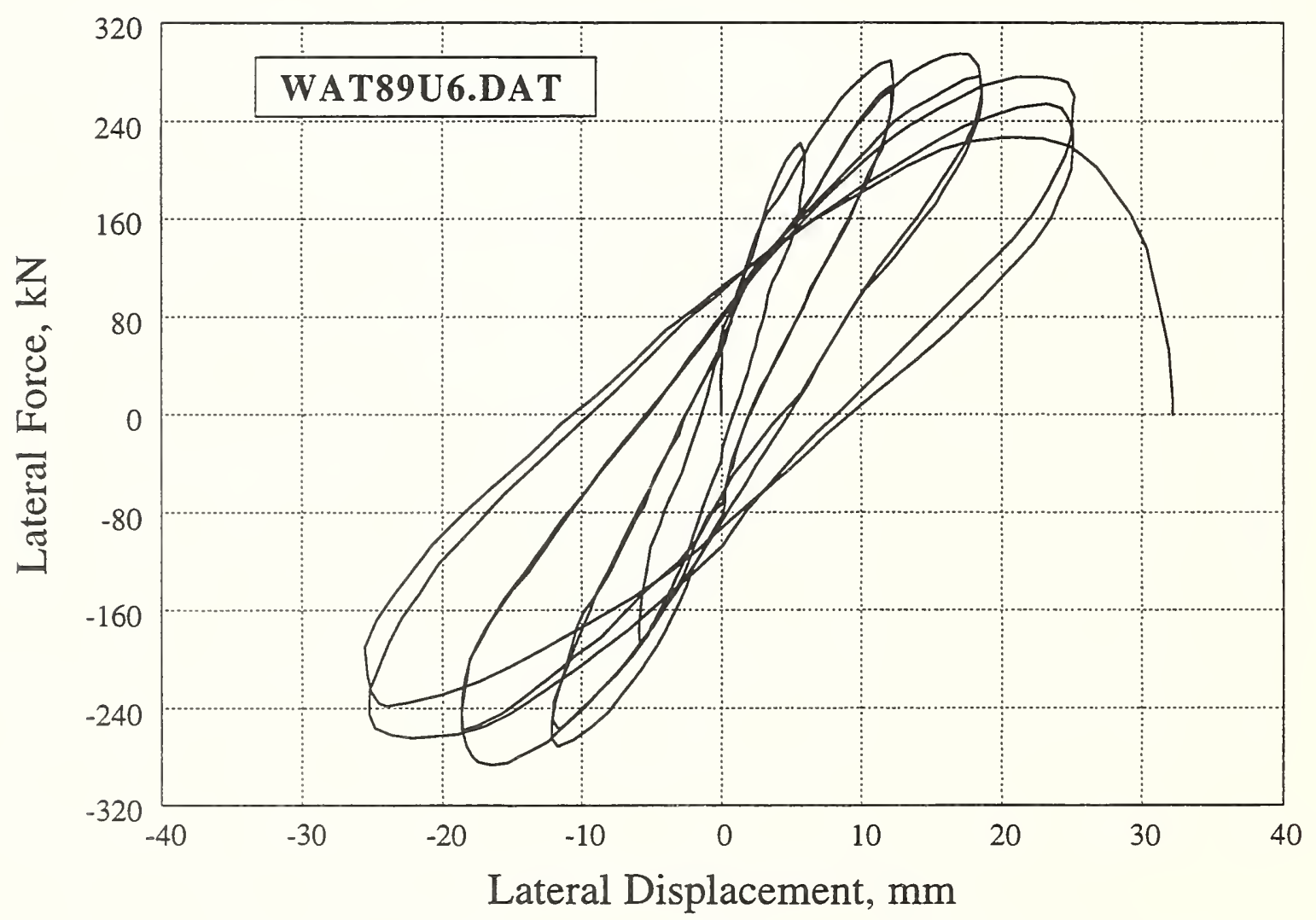

Figure 14. Specimen 6 of Watson 1989 


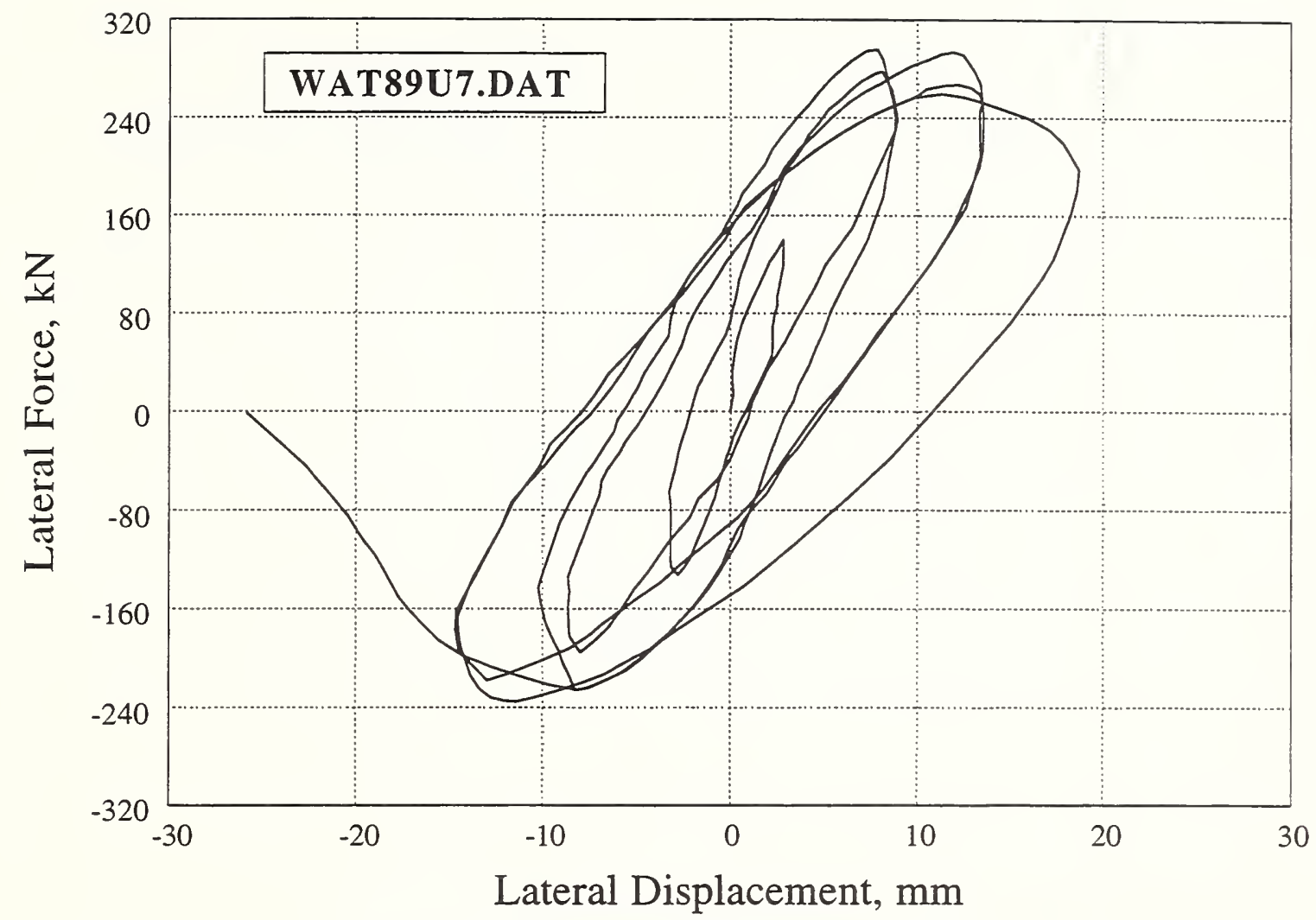

Figure 15. Specimen 7 of Watson 1989

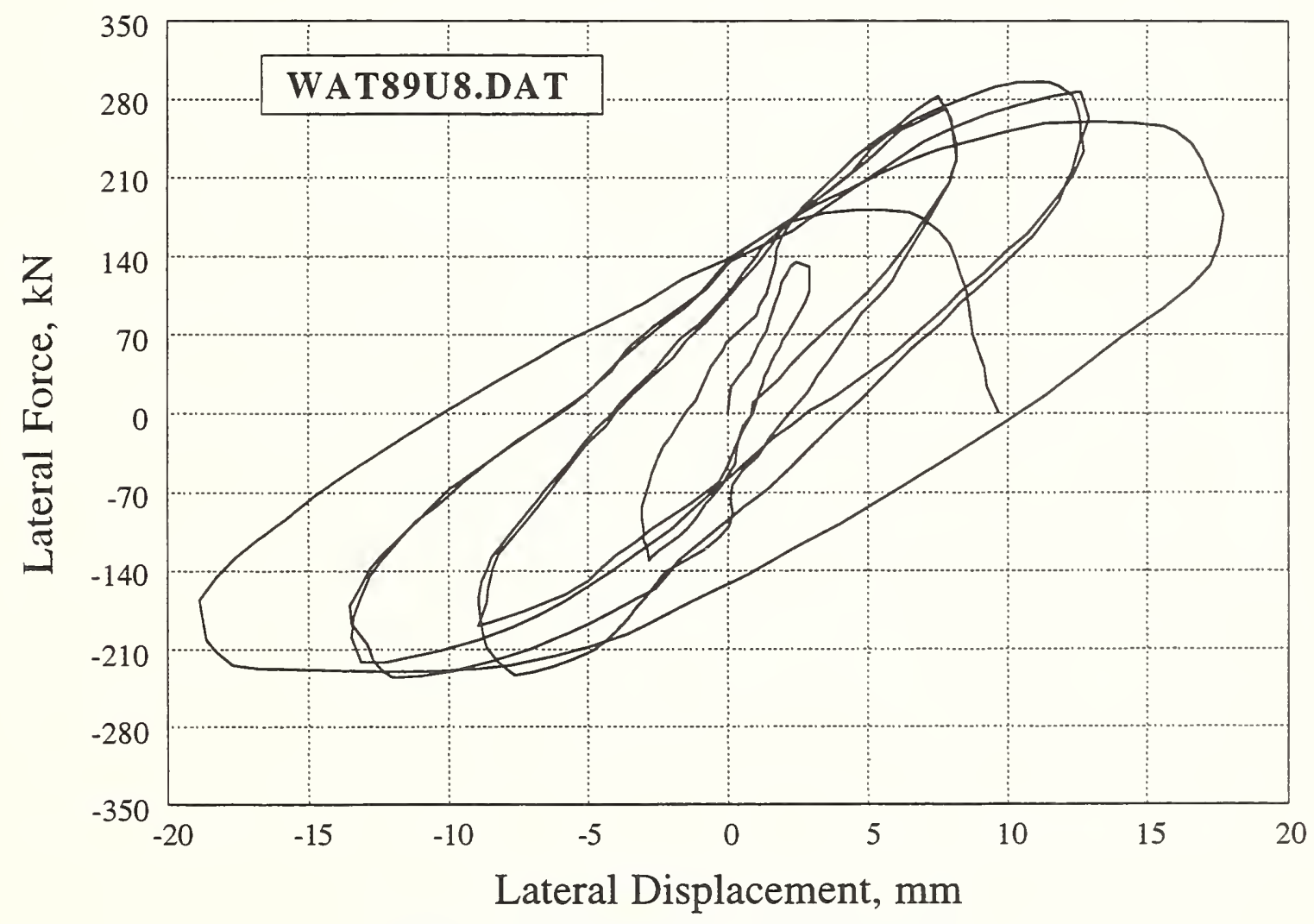

Figure 16. Specimen 8 of Watson 1989 


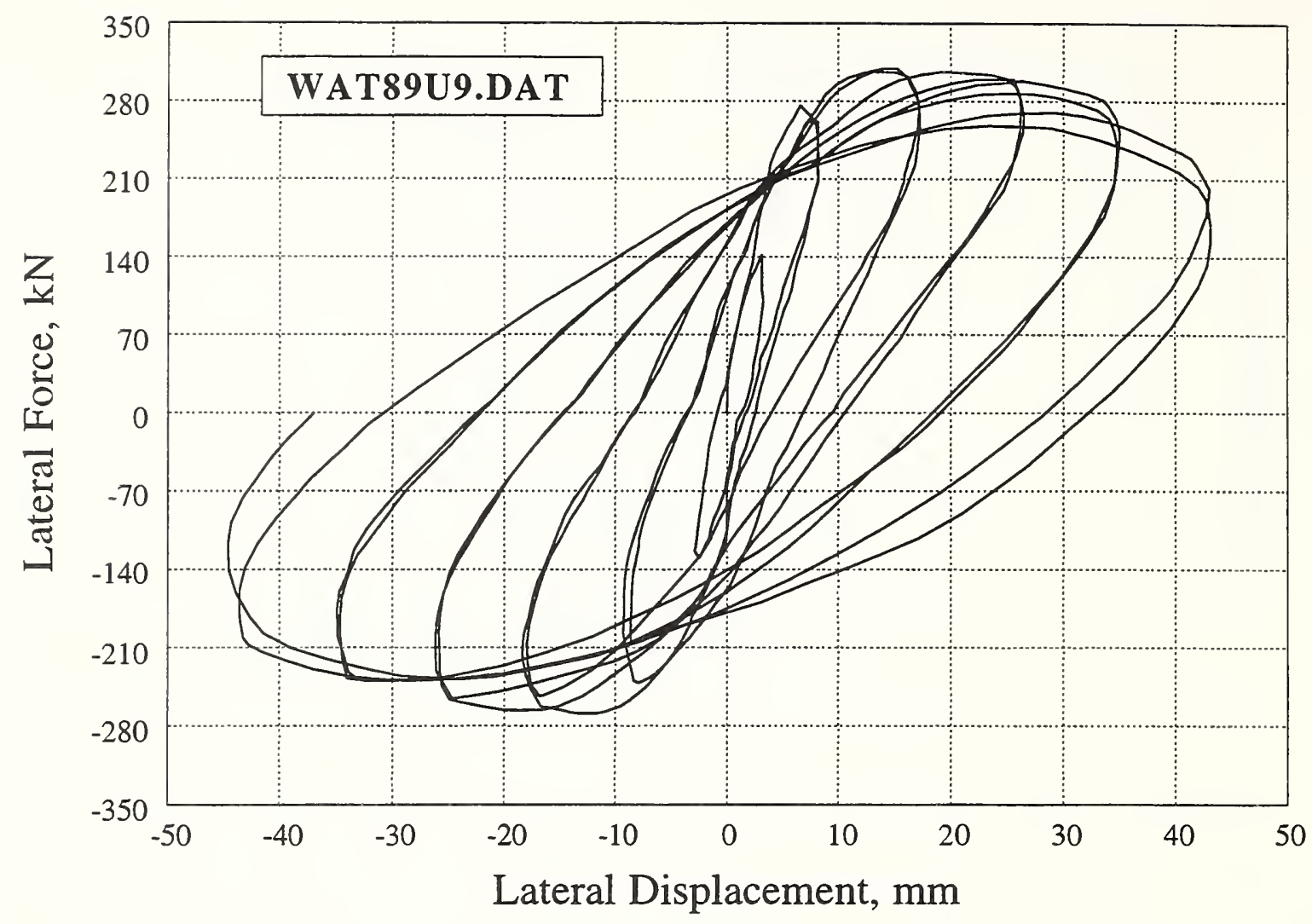

Figure 17. Specimen 9 of Watson 1989

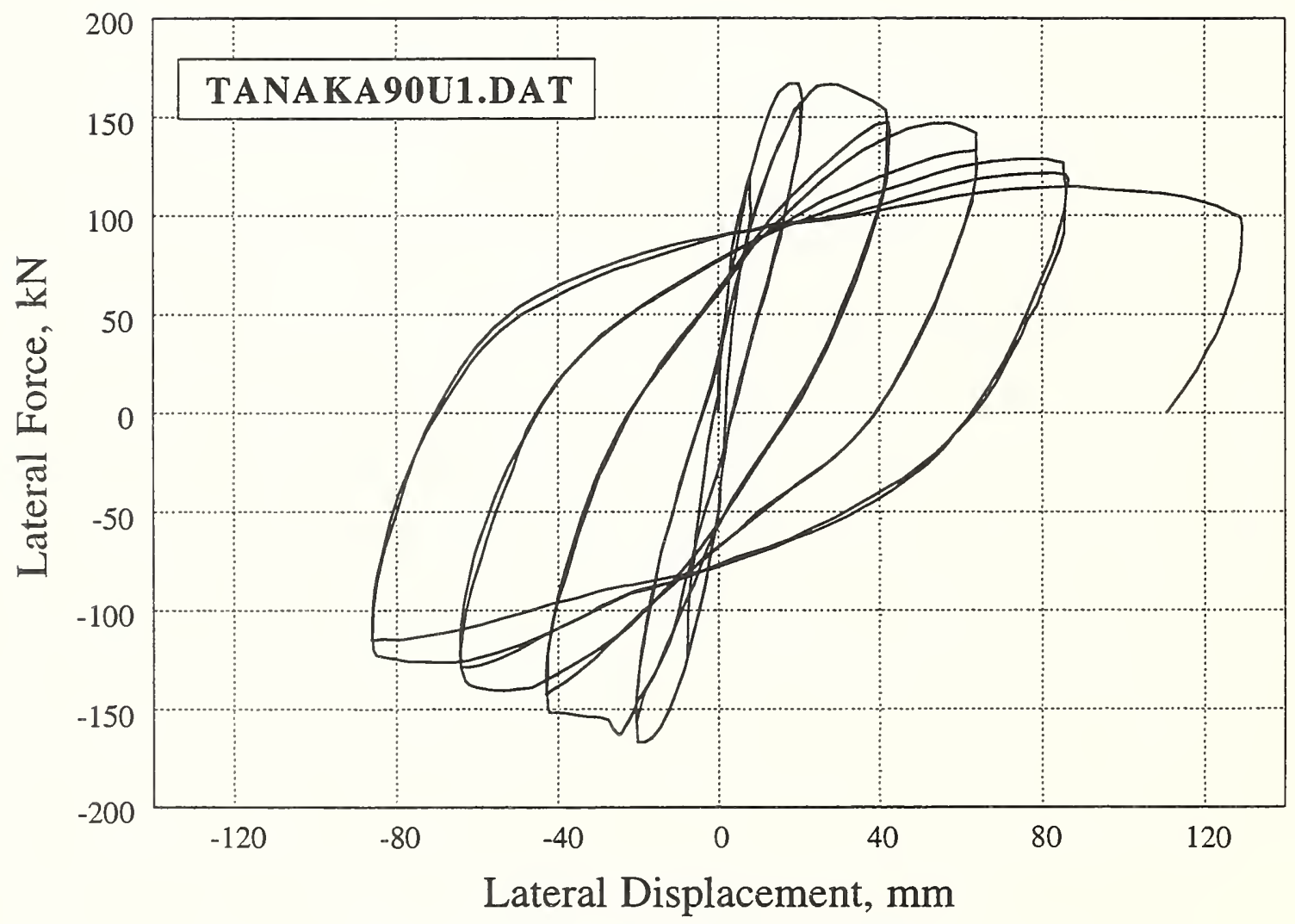

Figure 18. Specimen 1 of Tanaka 1990 


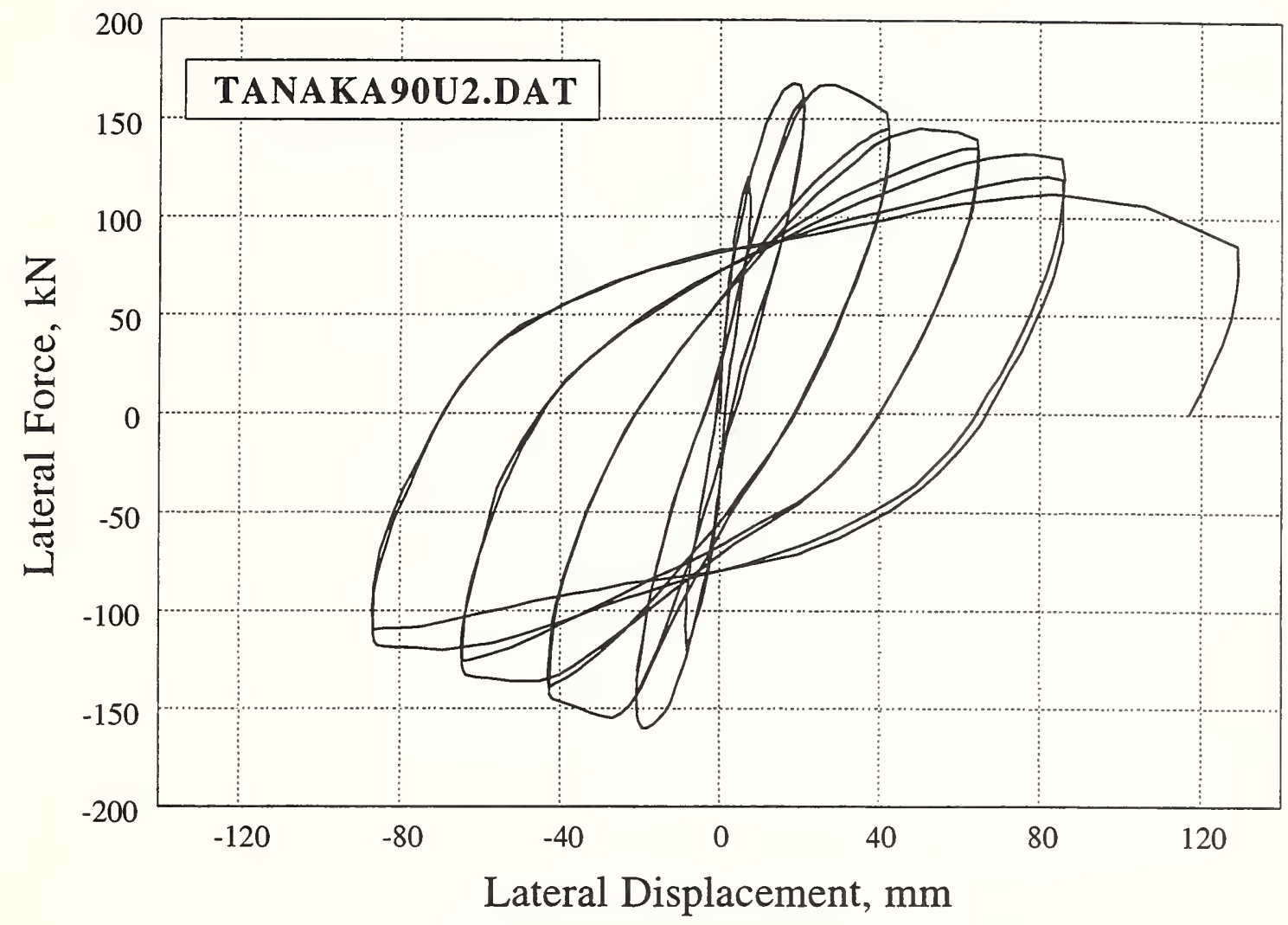

Figure 19. Specimen 2 of Tanaka 1990

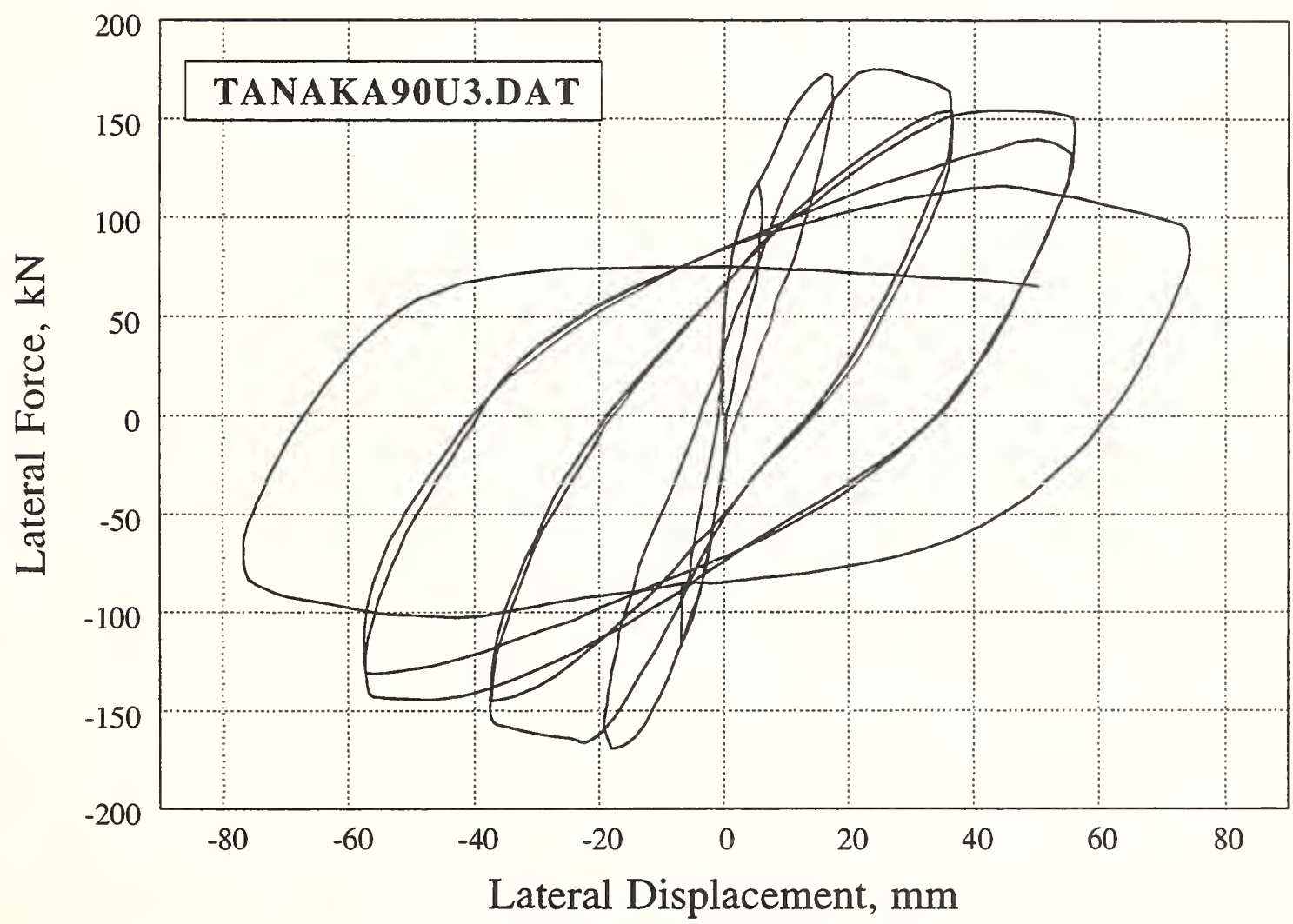

Figure 20. Specimen 3 of Tanaka 1990 


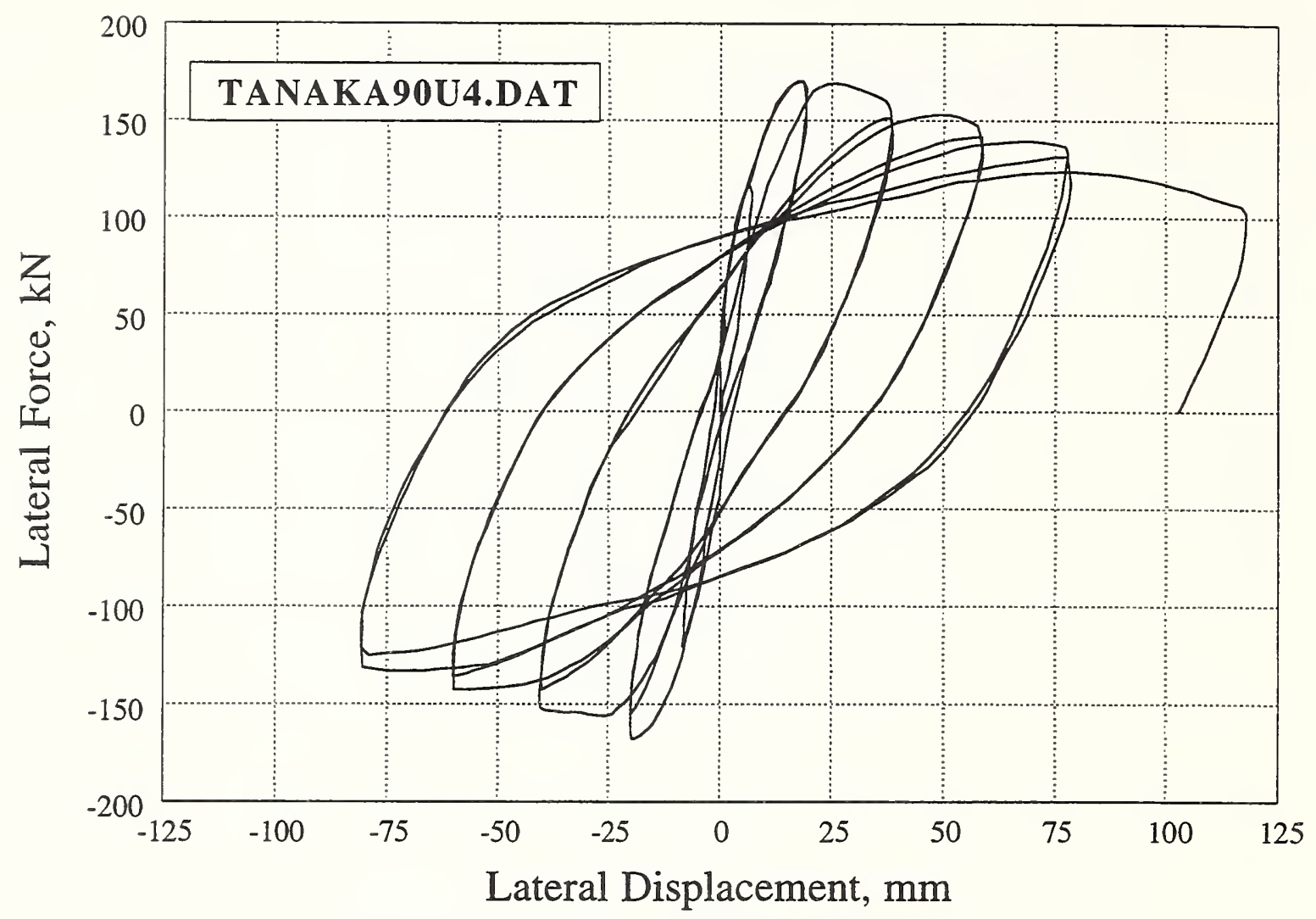

Figure 21. Specimen 4 of Tanaka 1990

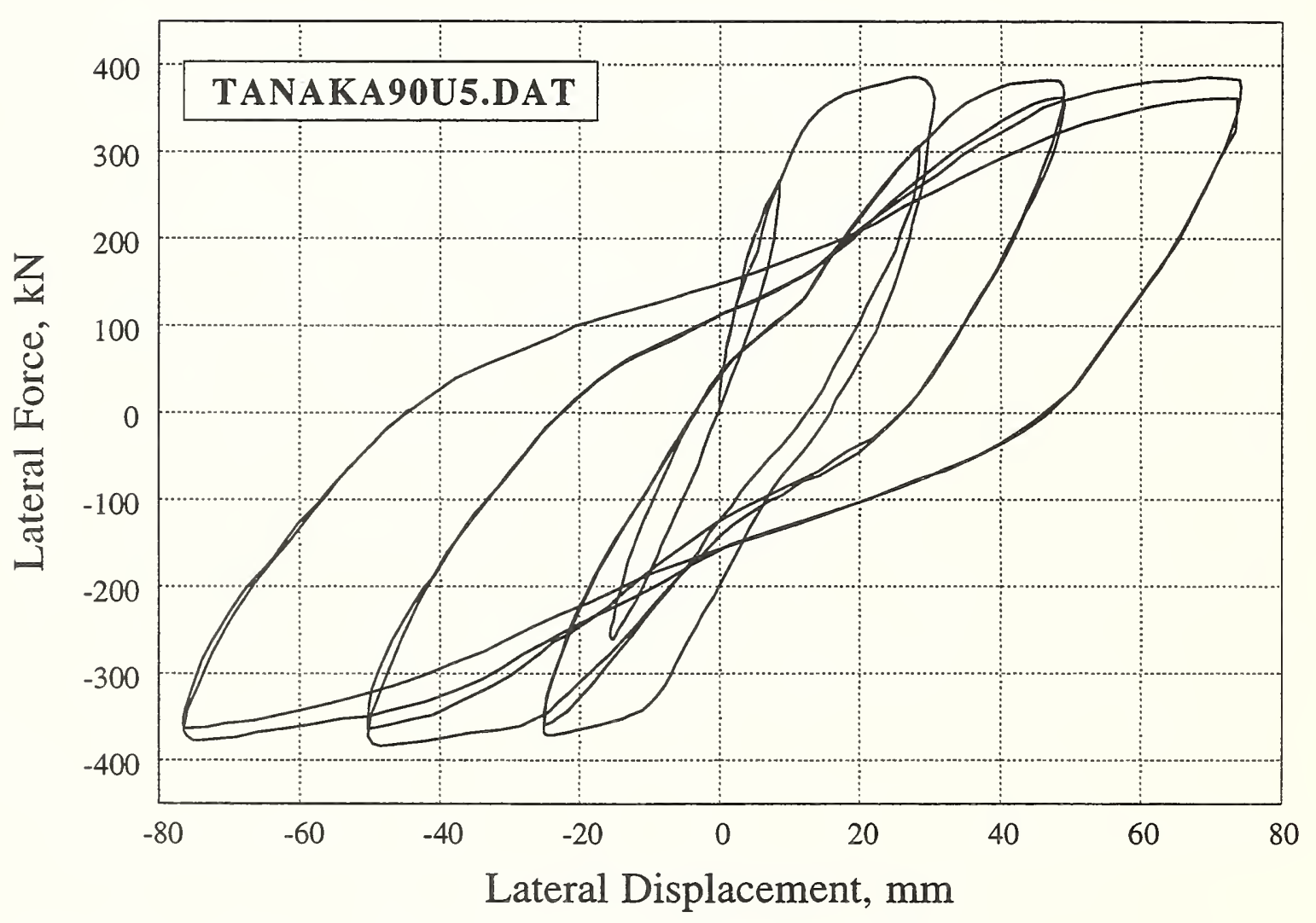

Figure 22. Specimen 5 of Tanaka 1990 


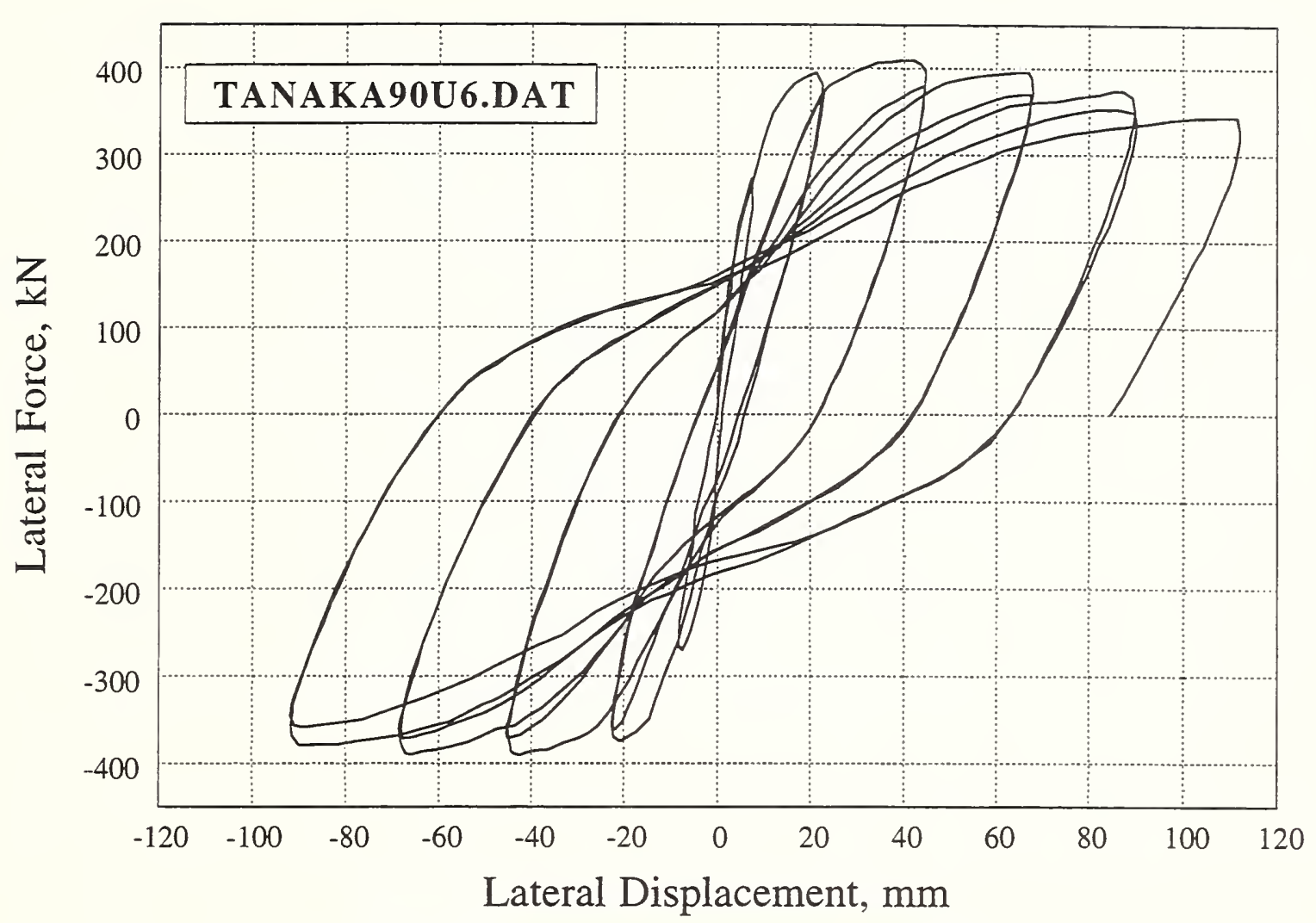

Figure 23. Specimen 6 of Tanaka 1990

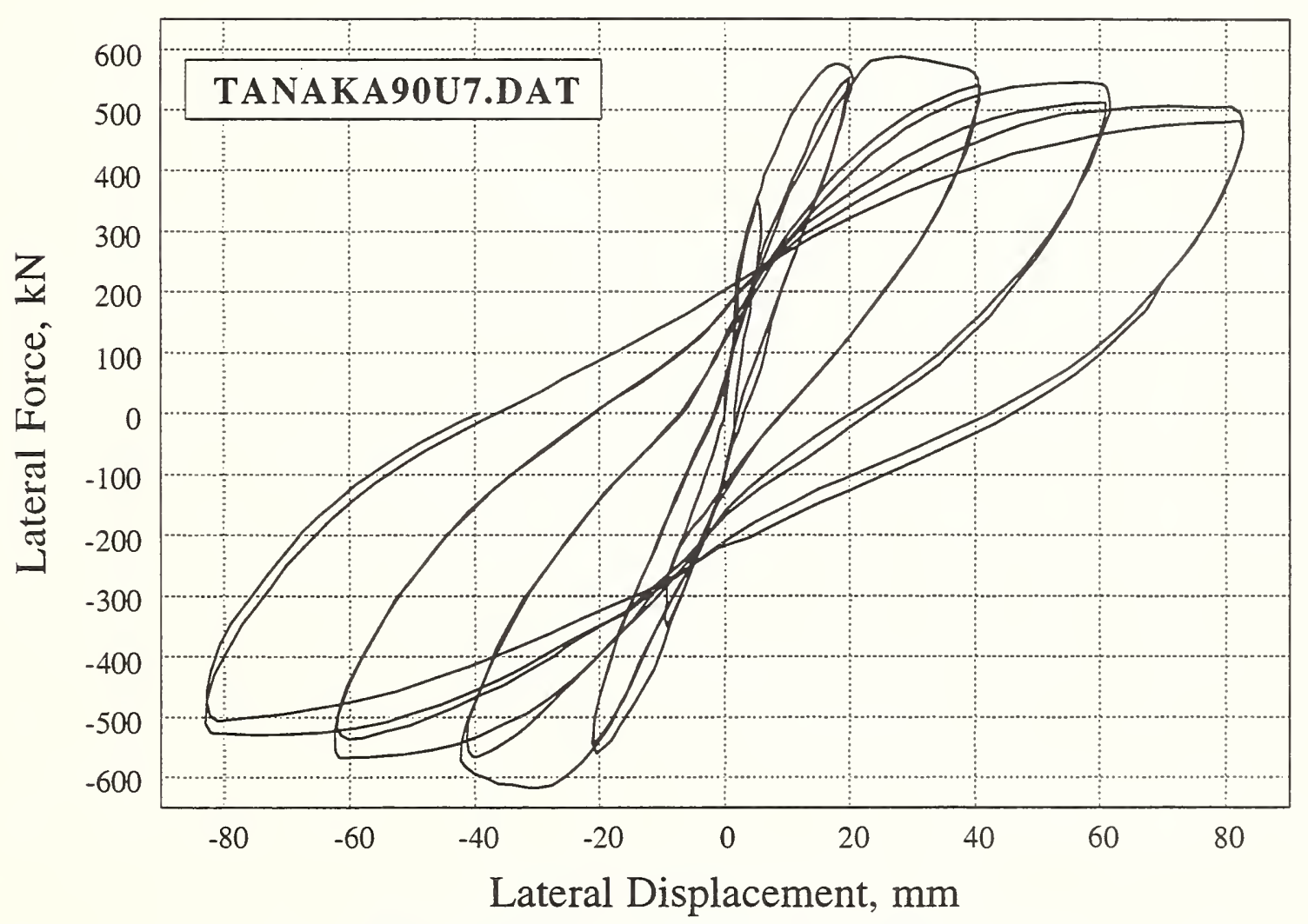

Figure 24. Specimen 7 of Tanaka 1990 


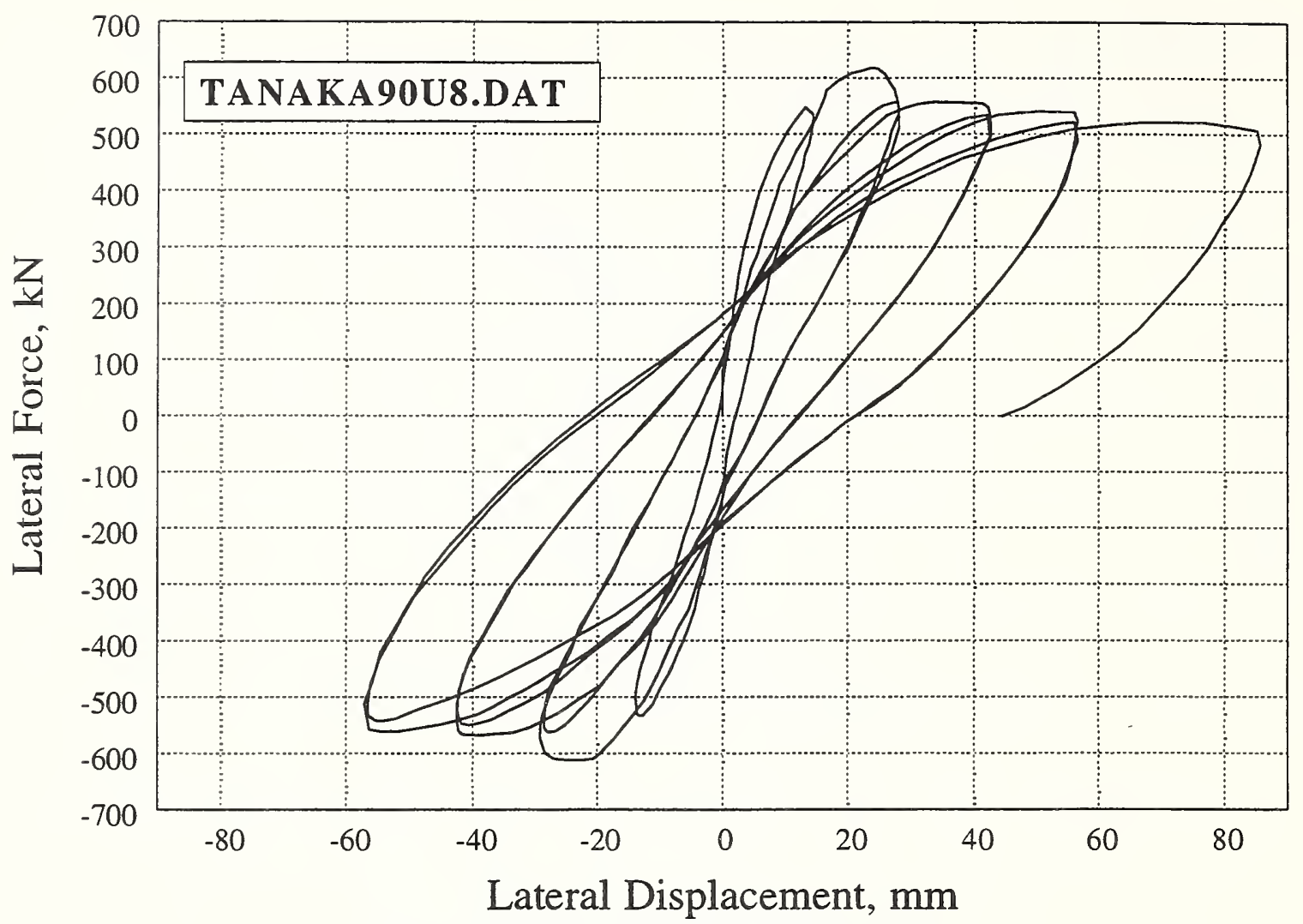

Figure 25. Specimen 8 of Tanaka 1990

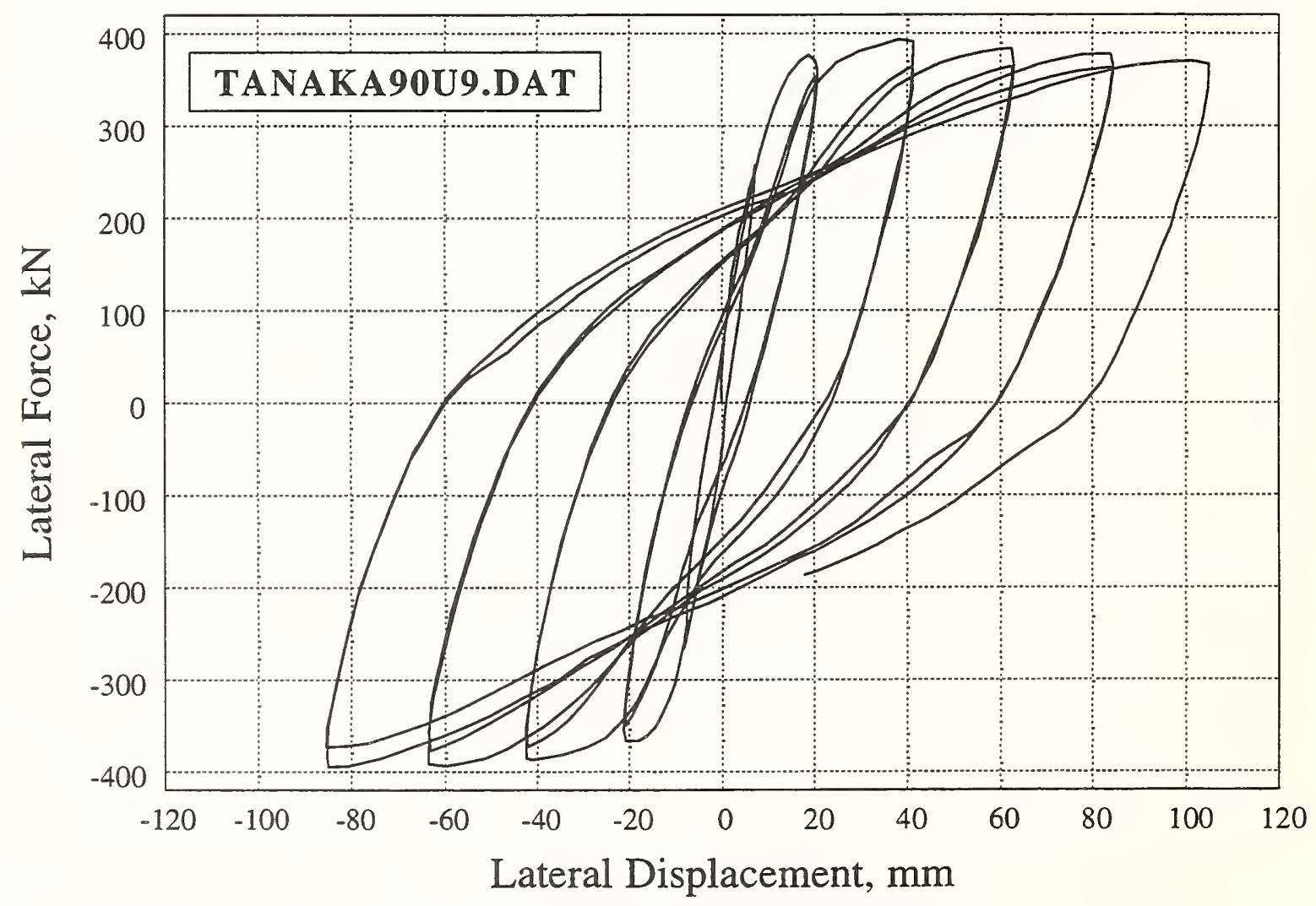

Figure 26. Specimen 9 of Tanaka 1990 


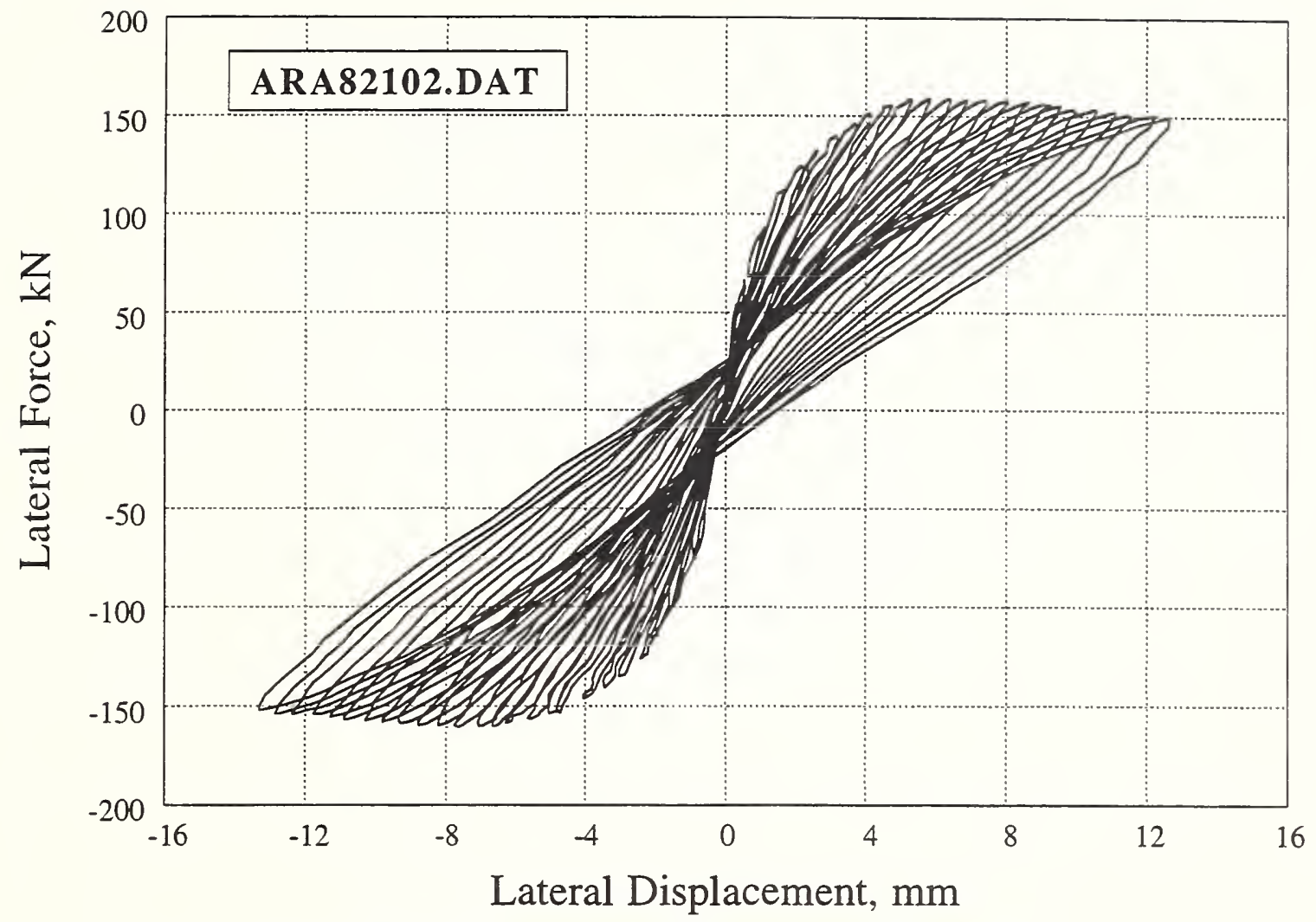

Figure 27. Specimen 102 of Arakawa 1982

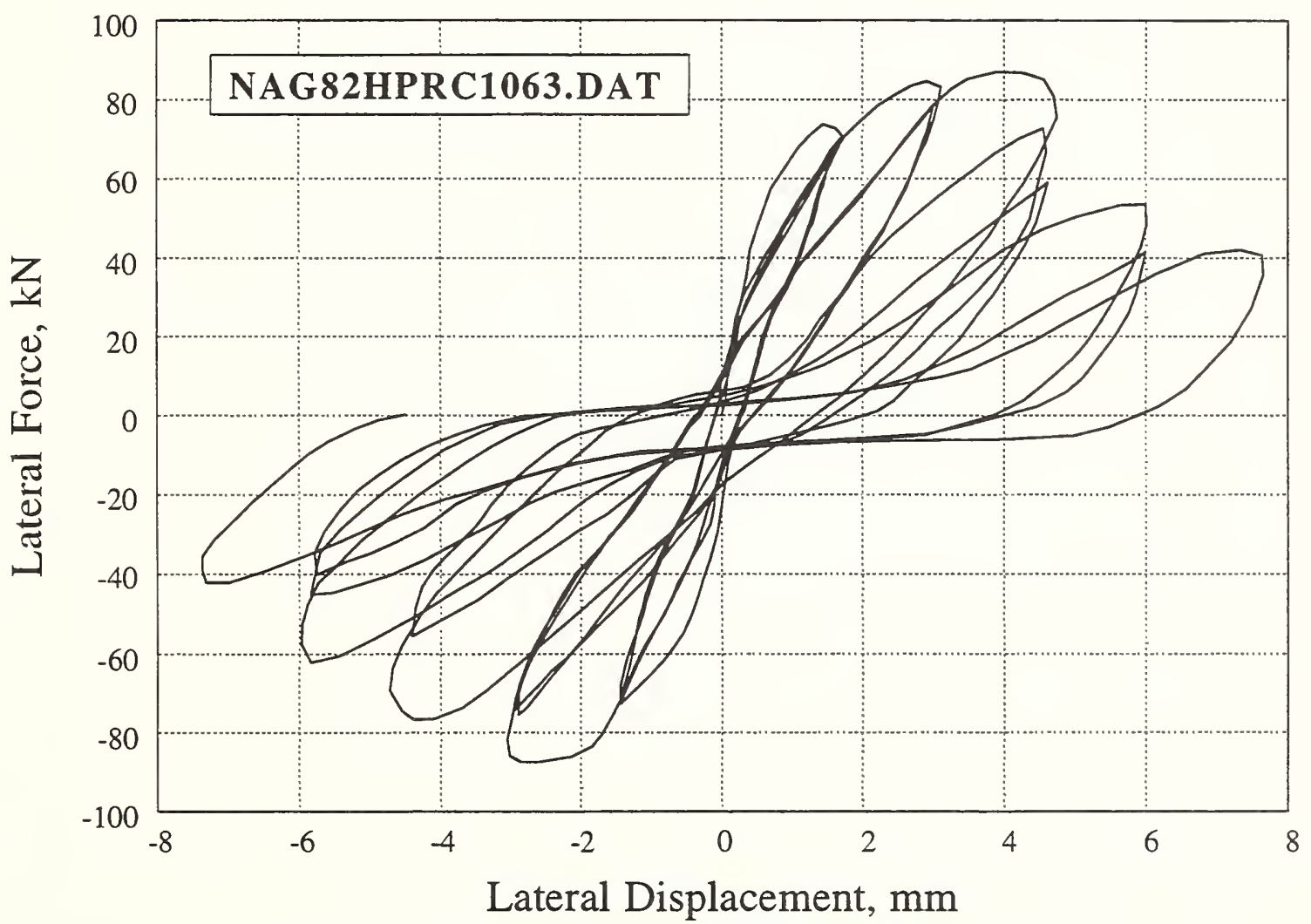

Fi gure 28. Specimen HPRC 10-63 of Nagasaka 1982 


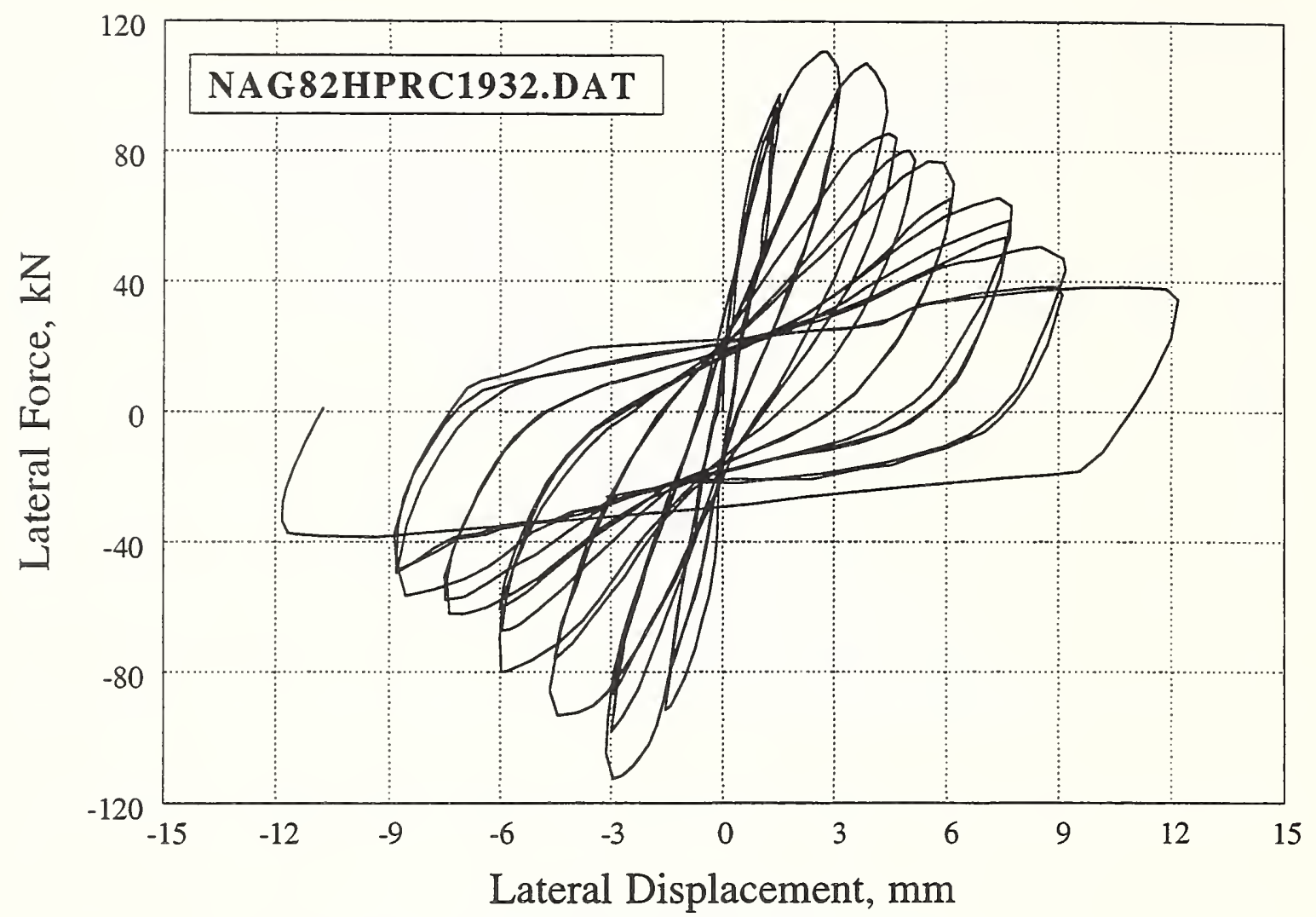

Figure 29. Specimen HPRC 19-32 of Nagasaka 1982

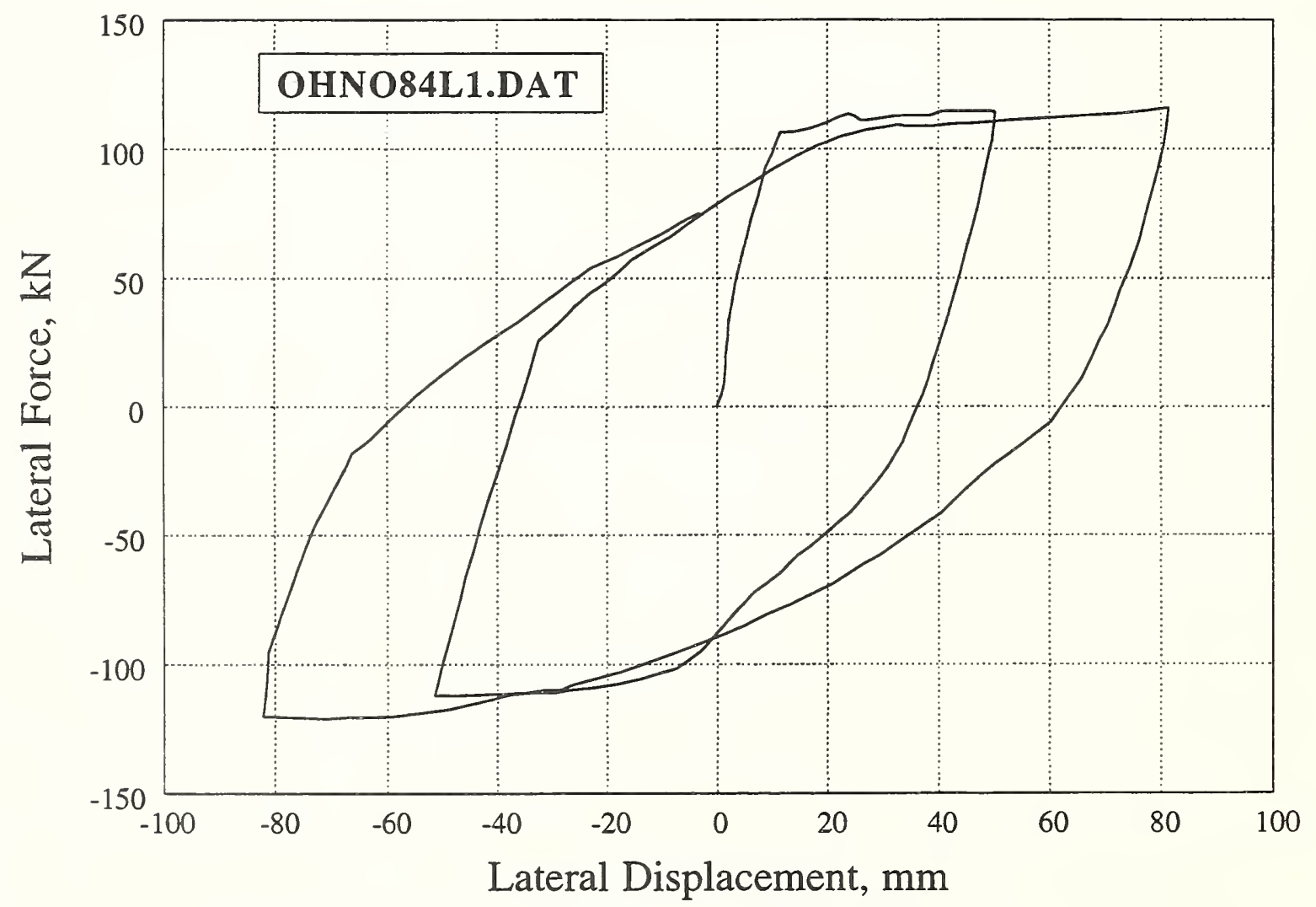

Figure 30. Specimen L1 of Ohno 1984 


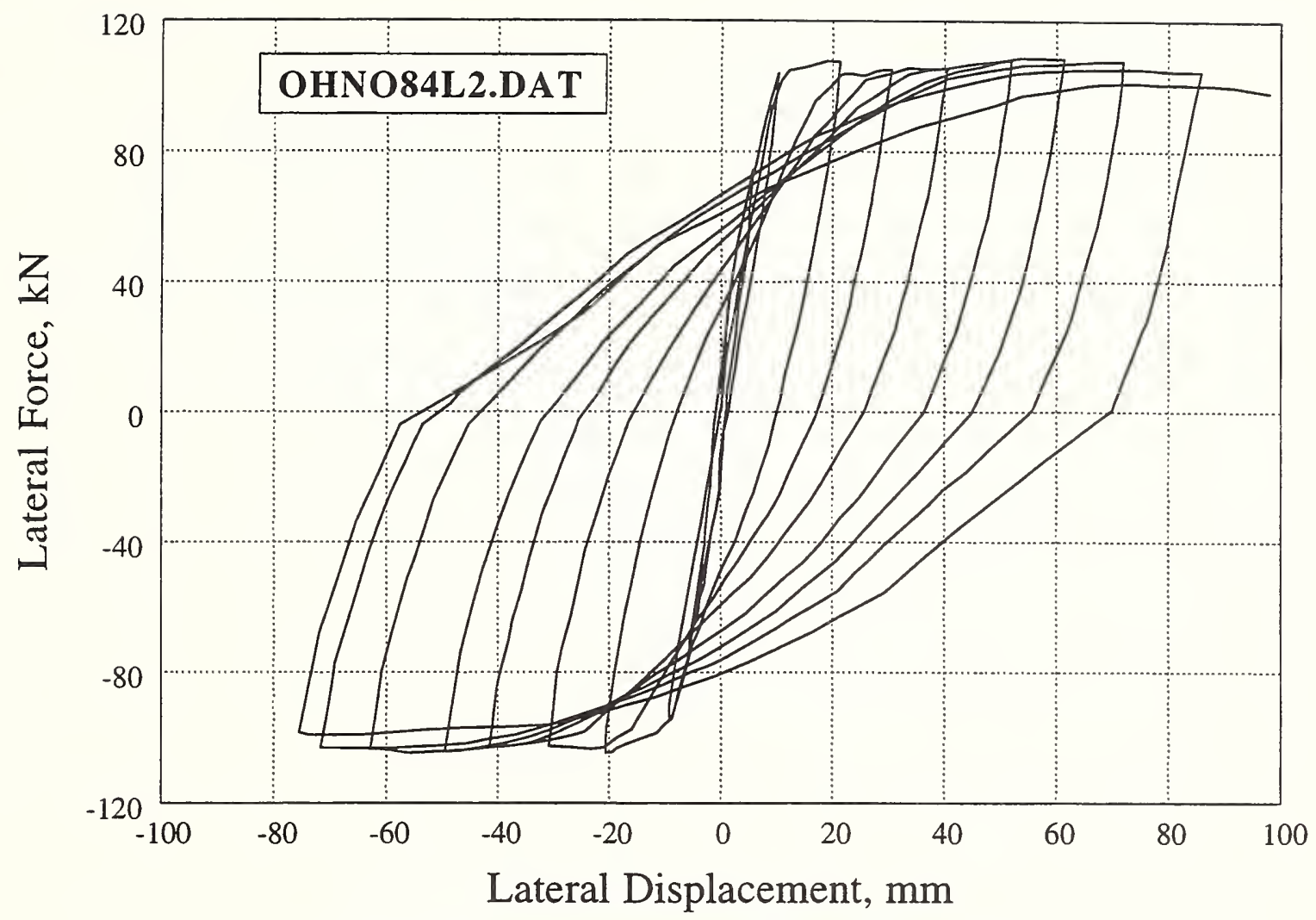

Figure 31. Specimen L2 of Ohno 1984

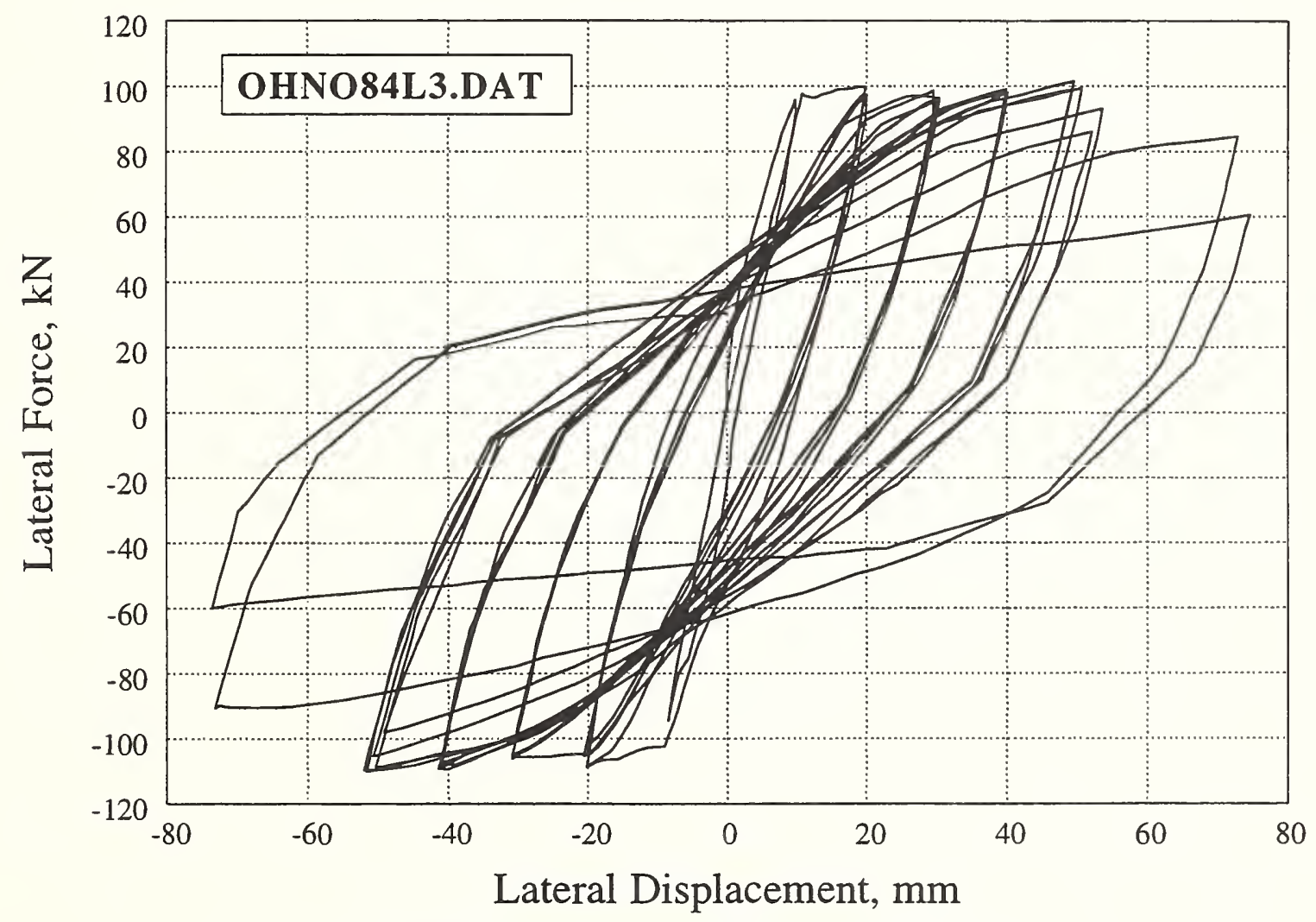

Figure 32. Specimen L3 of Ohno 1984 


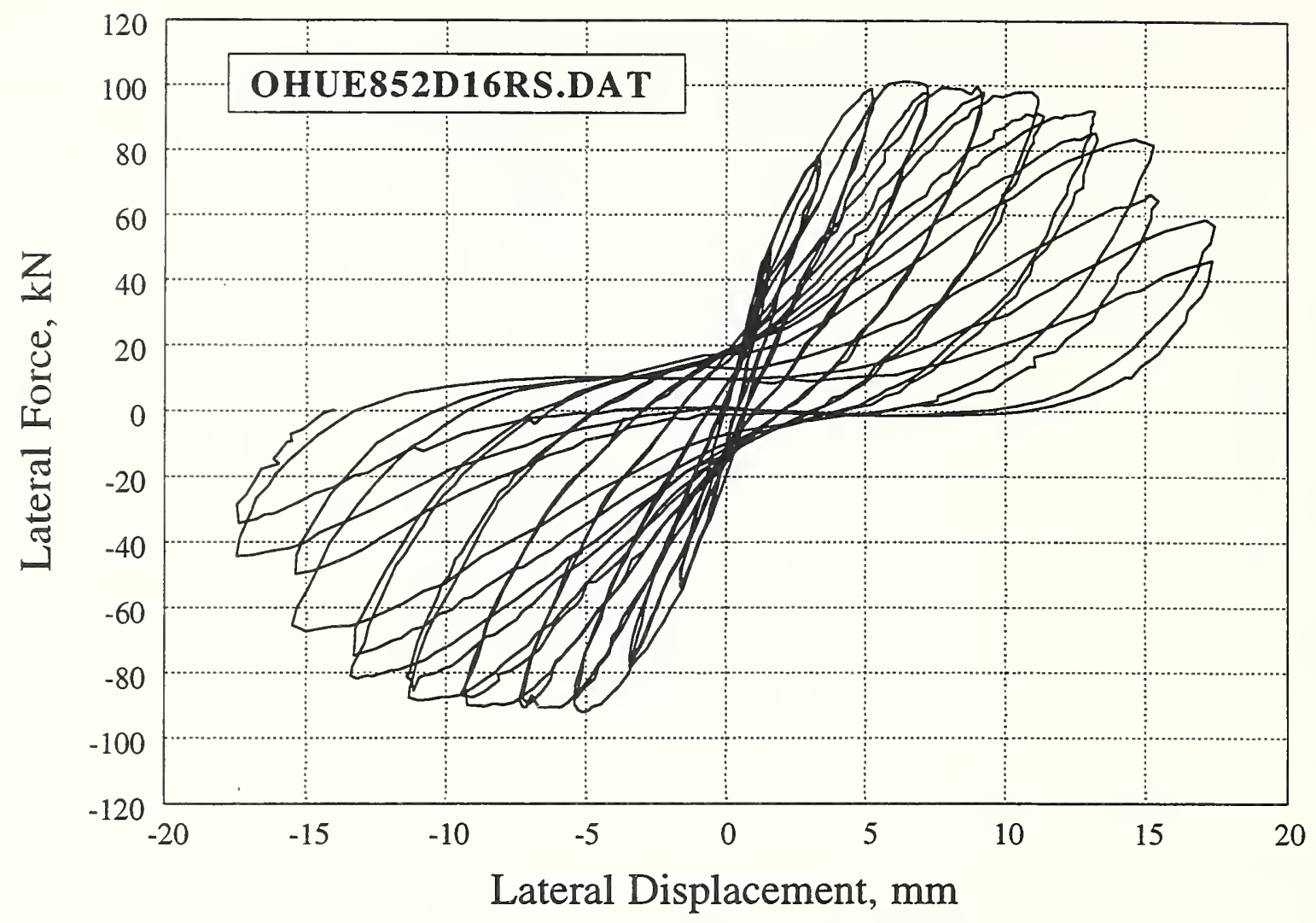

Figure 33. Specimen 2D16RS of Ohue 1985

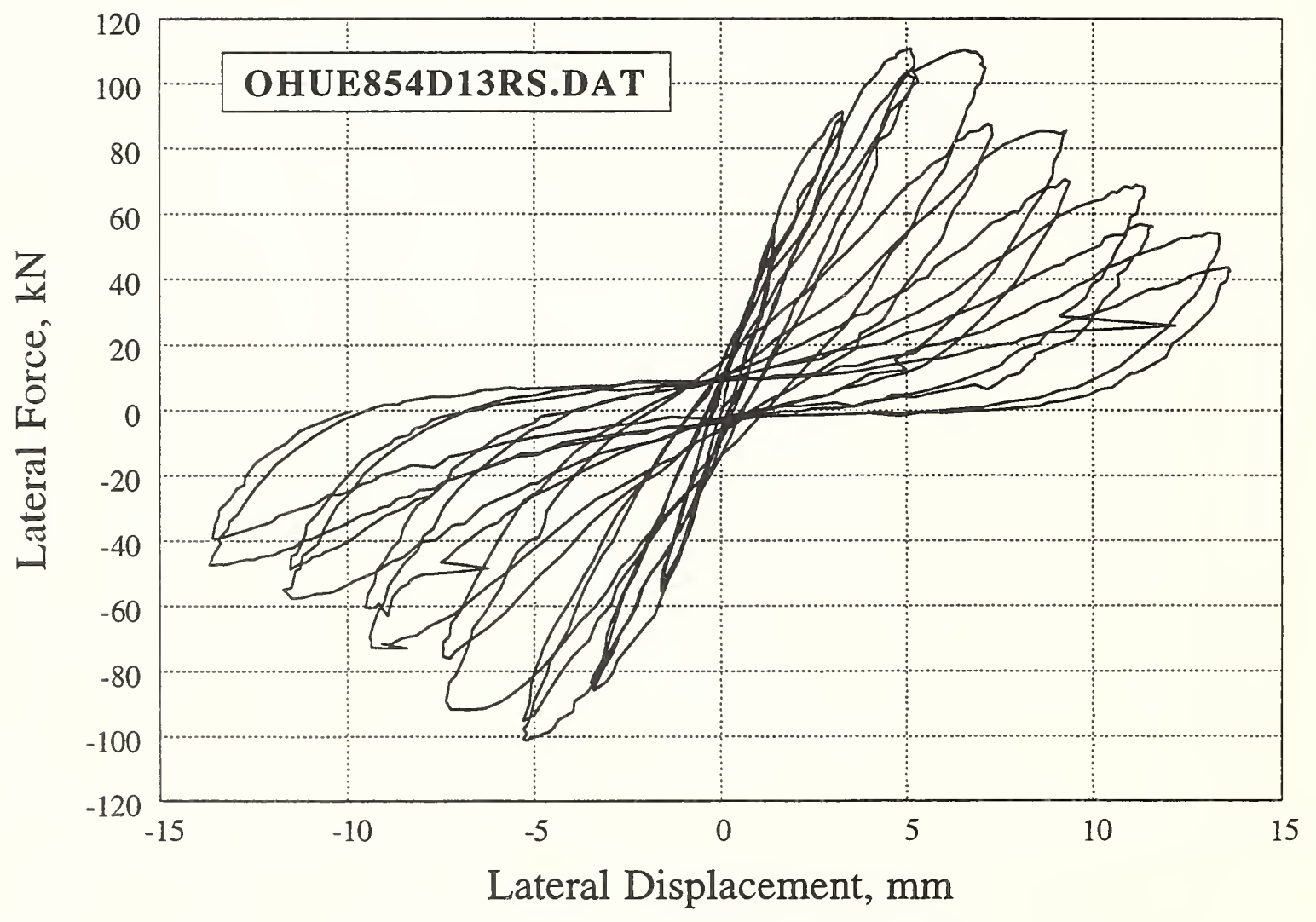

Figure 34. Specimen 4D13RS of Ohue 1985 


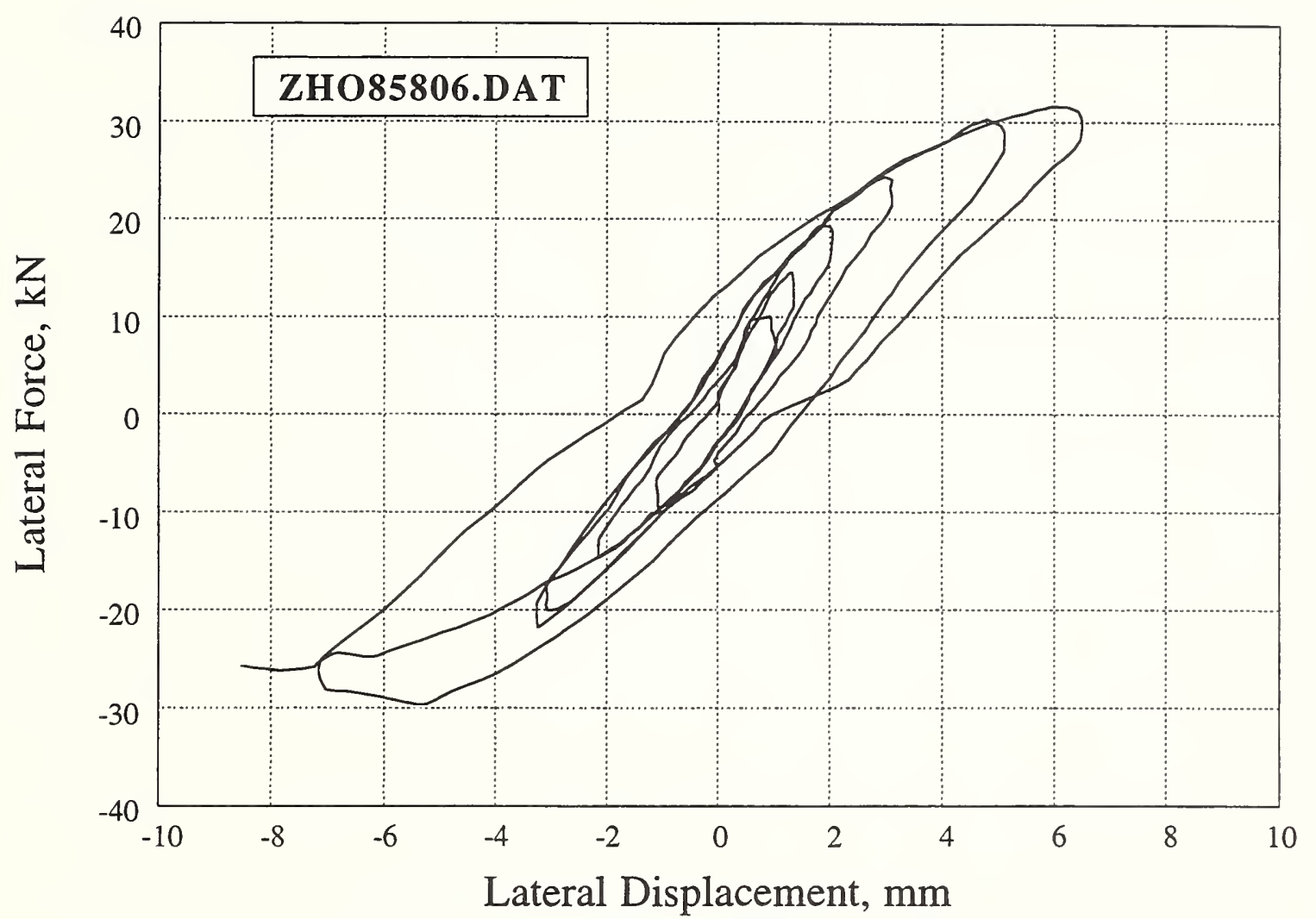

Figure 35. Specimen AZ8-06 of Zhou et al. 1985

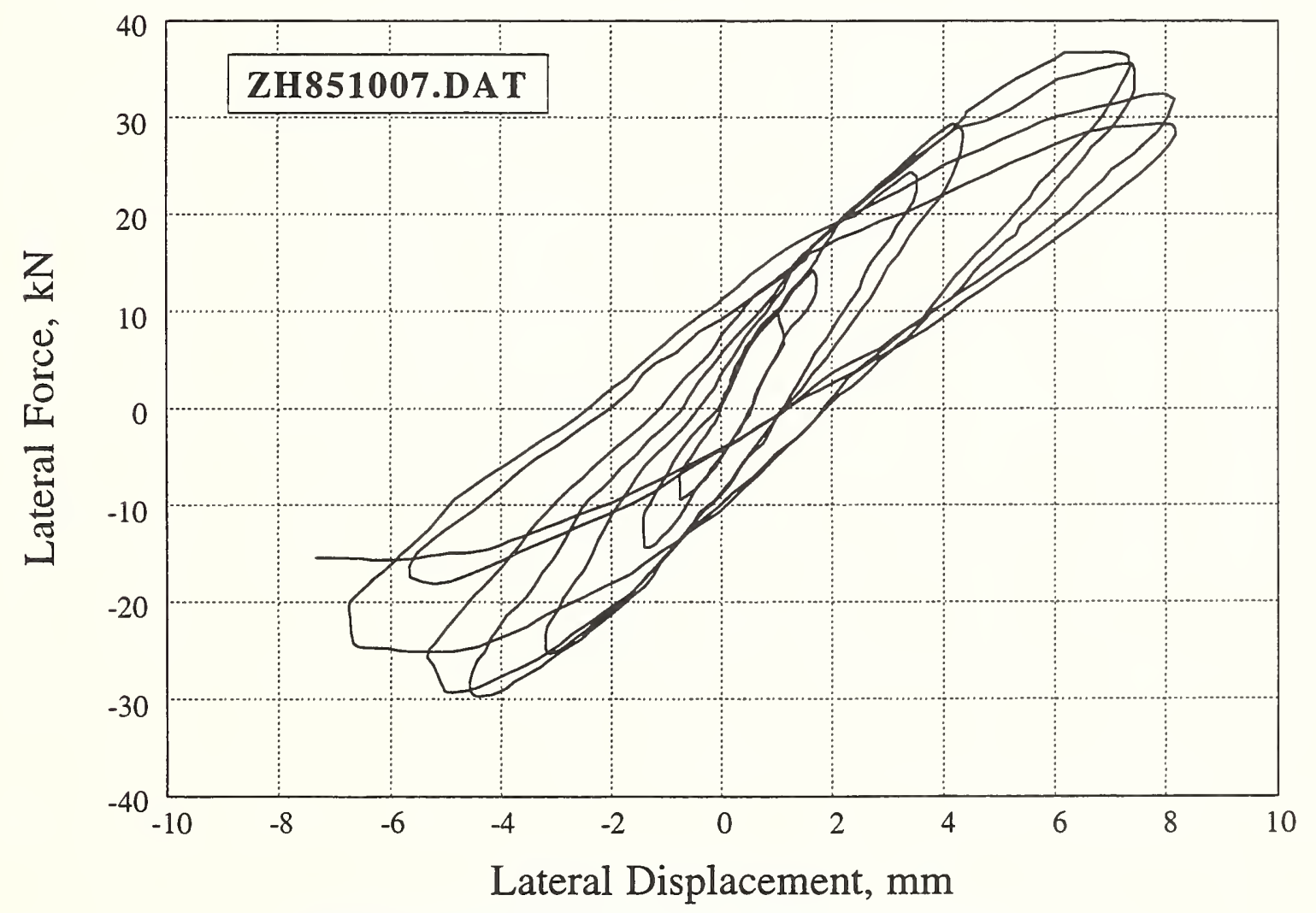

Figure 36. Specimen AZ10-07 of Zhou et al. 1985 


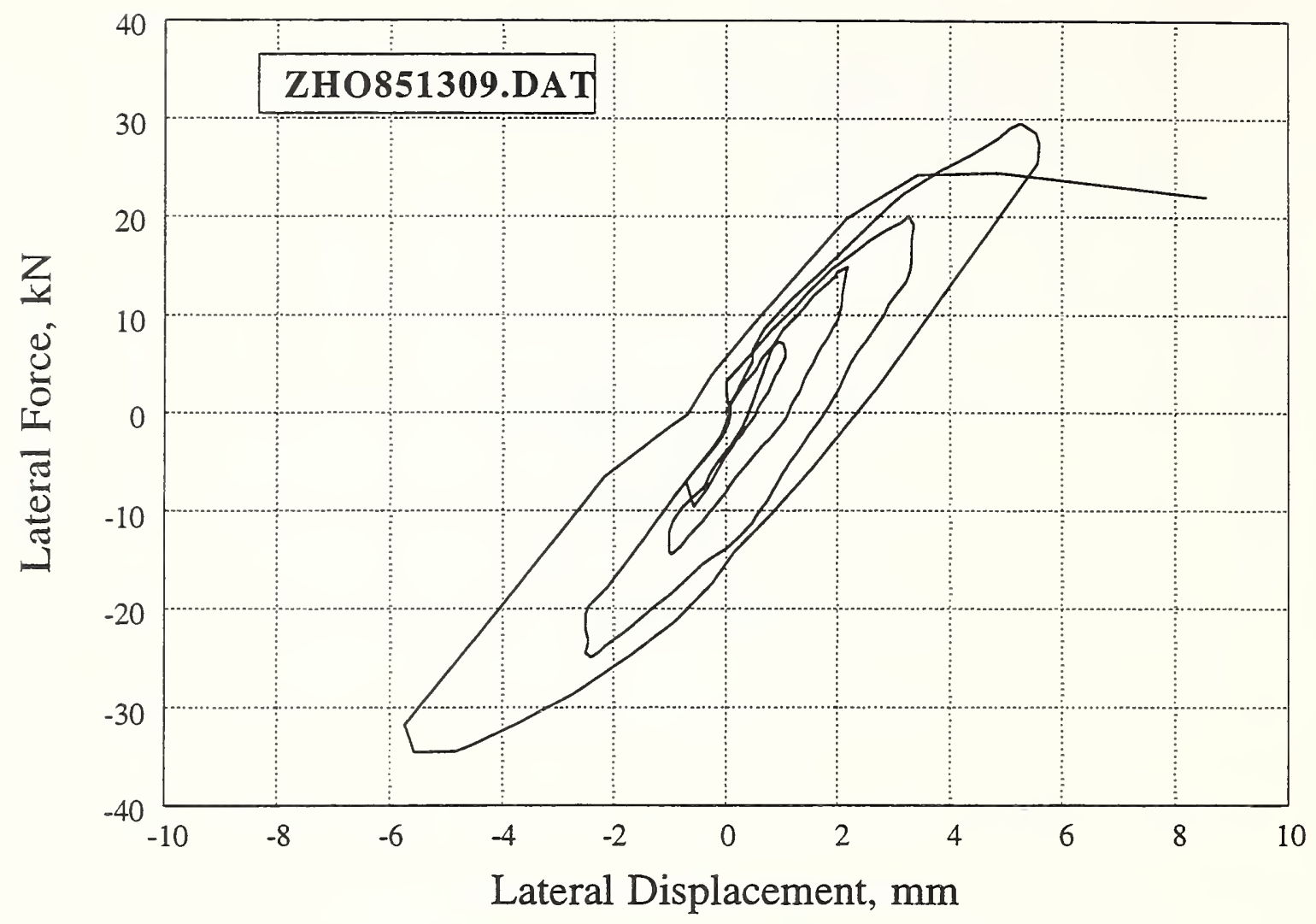

Figure 37. Specimen AZ13-09 of Zhou et al. 1985

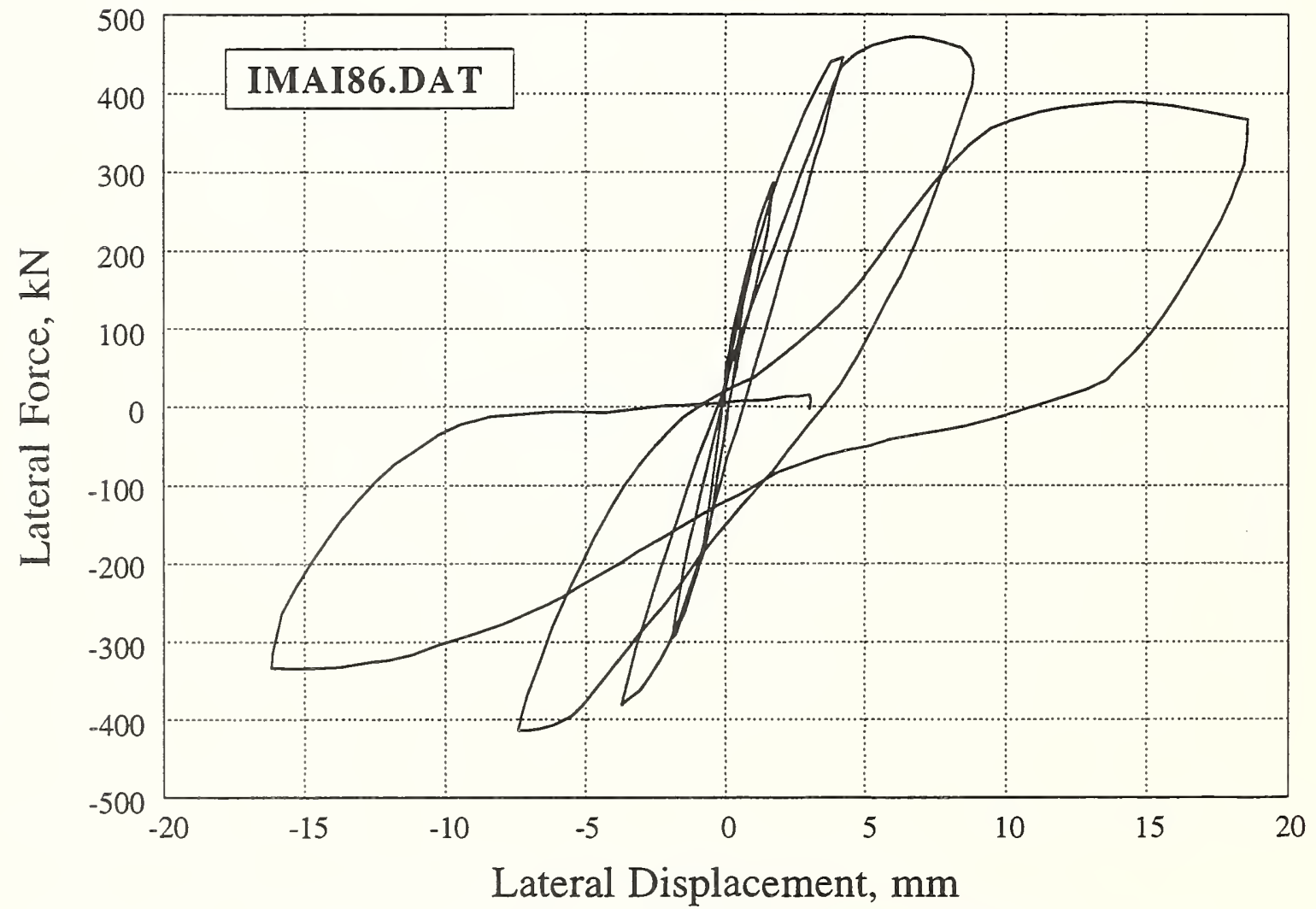

Figure 38. Specimen 1 of Imai and Yamamoto 1986 


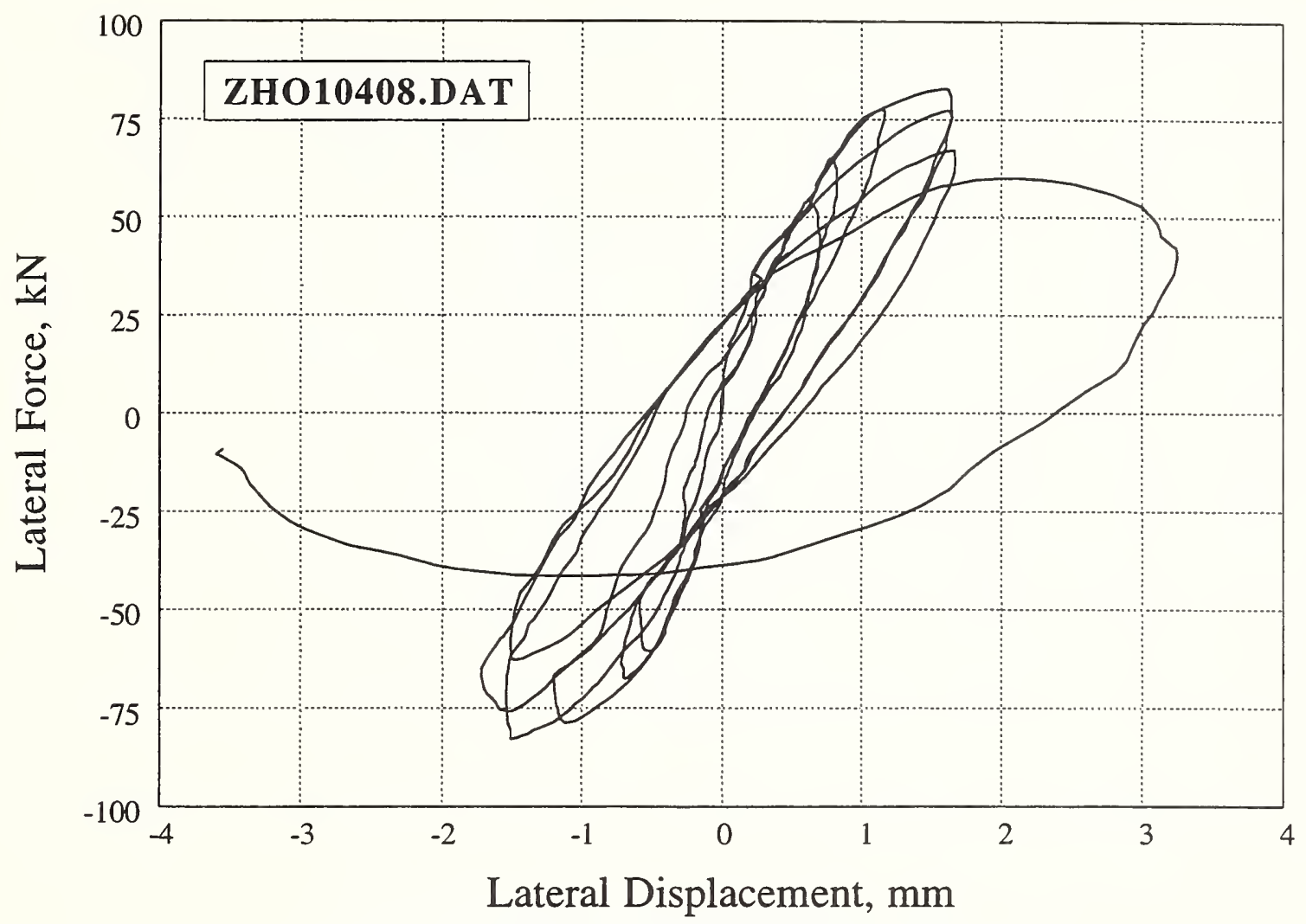

Figure 39. Specimen 104-08 of Zhou et al. 1987

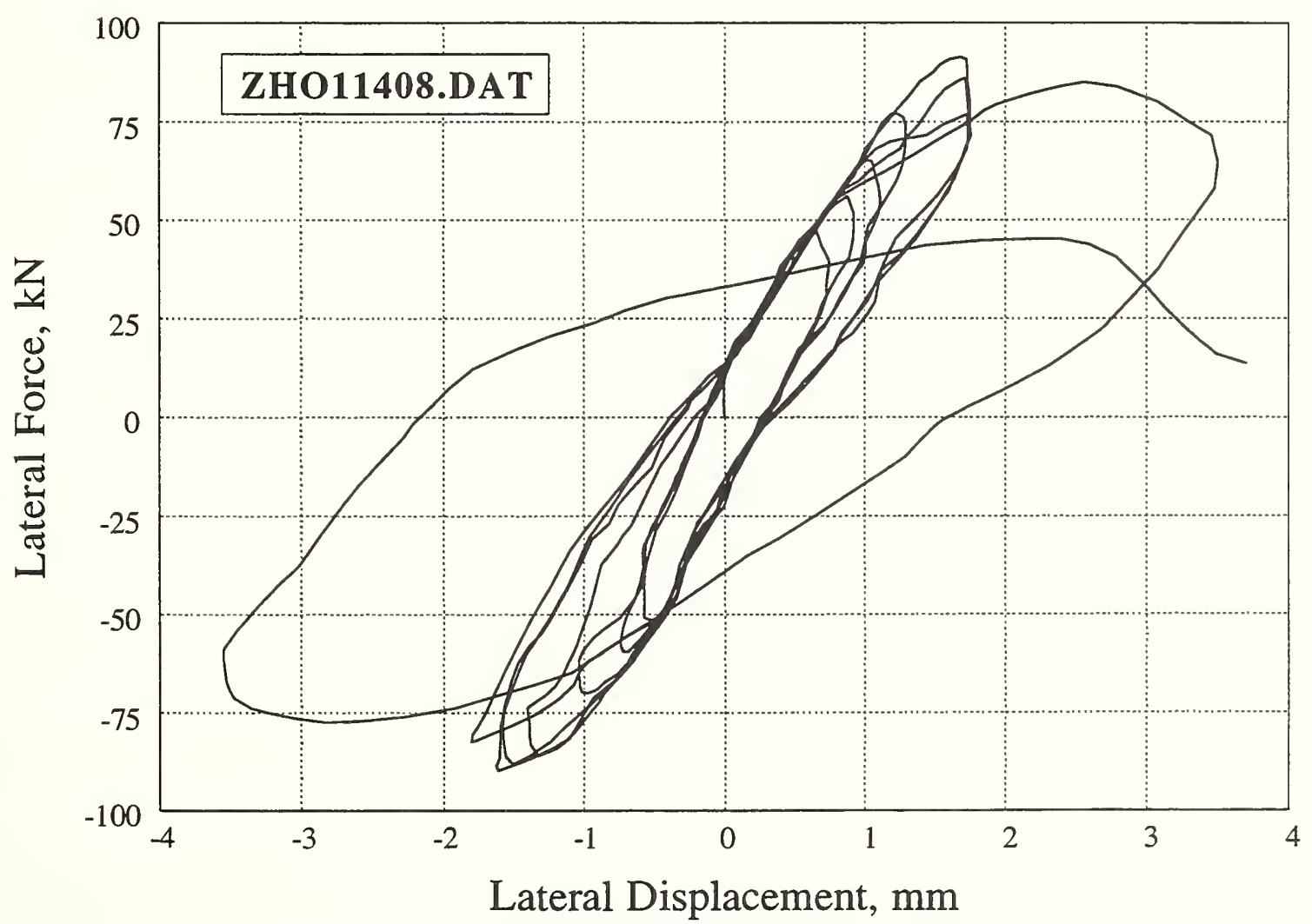

Figure 40. Specimen 114-08 of Zhou et al. 1987 


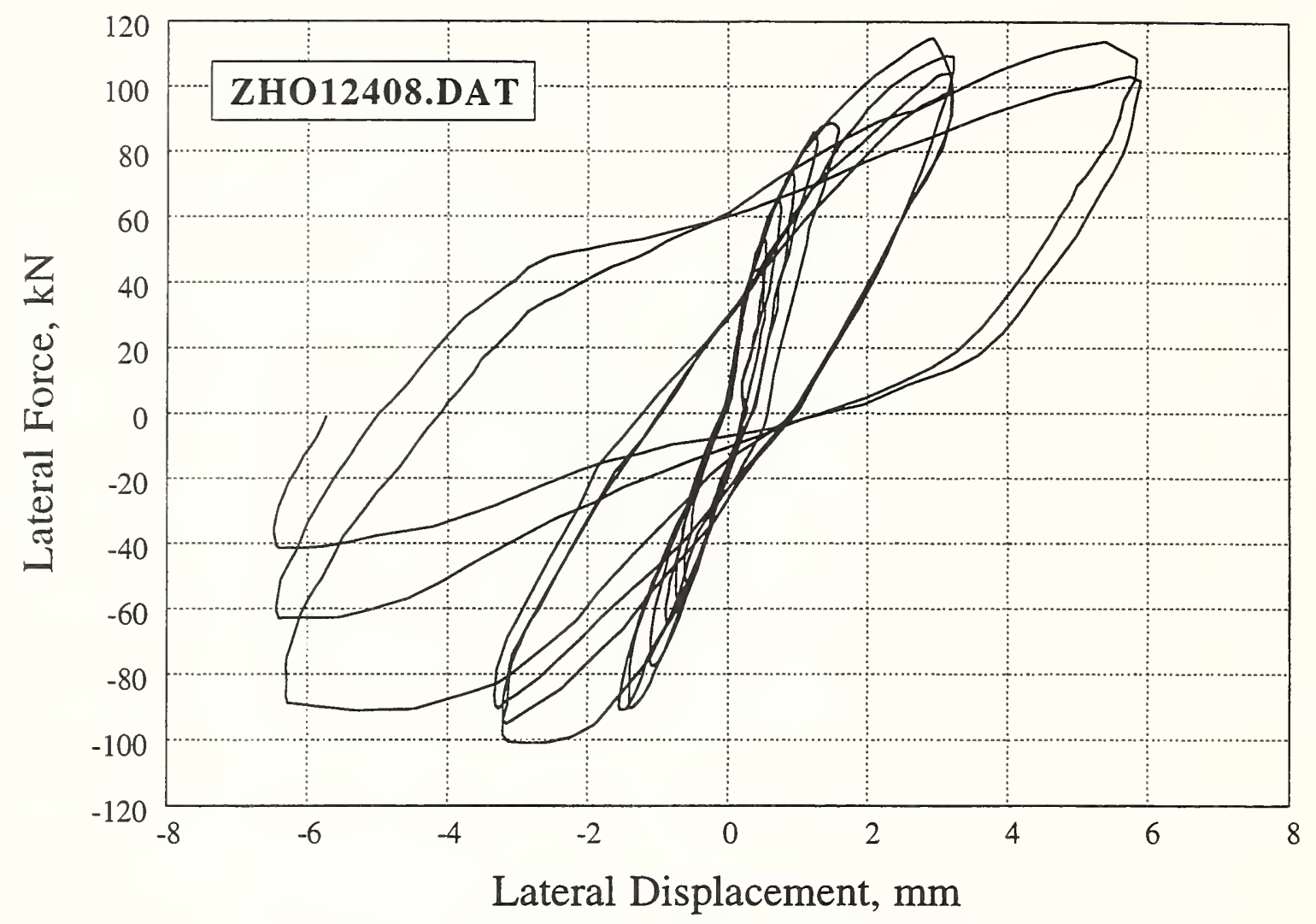

Figure 41. Specimen 124-08 of Zhou et al. 1987

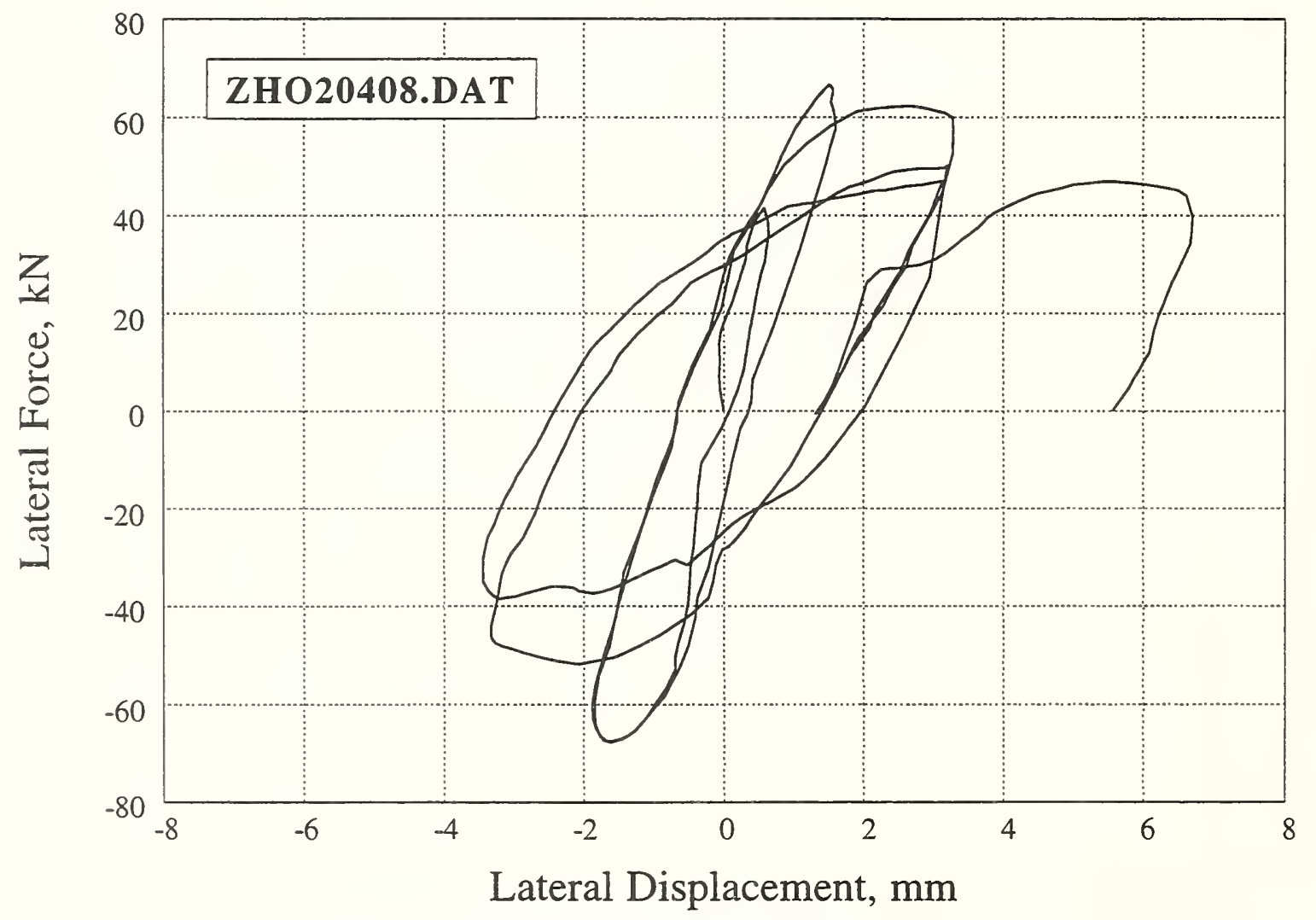

Figure 42. Specimen 204-08 of Zhou et al. 1987 


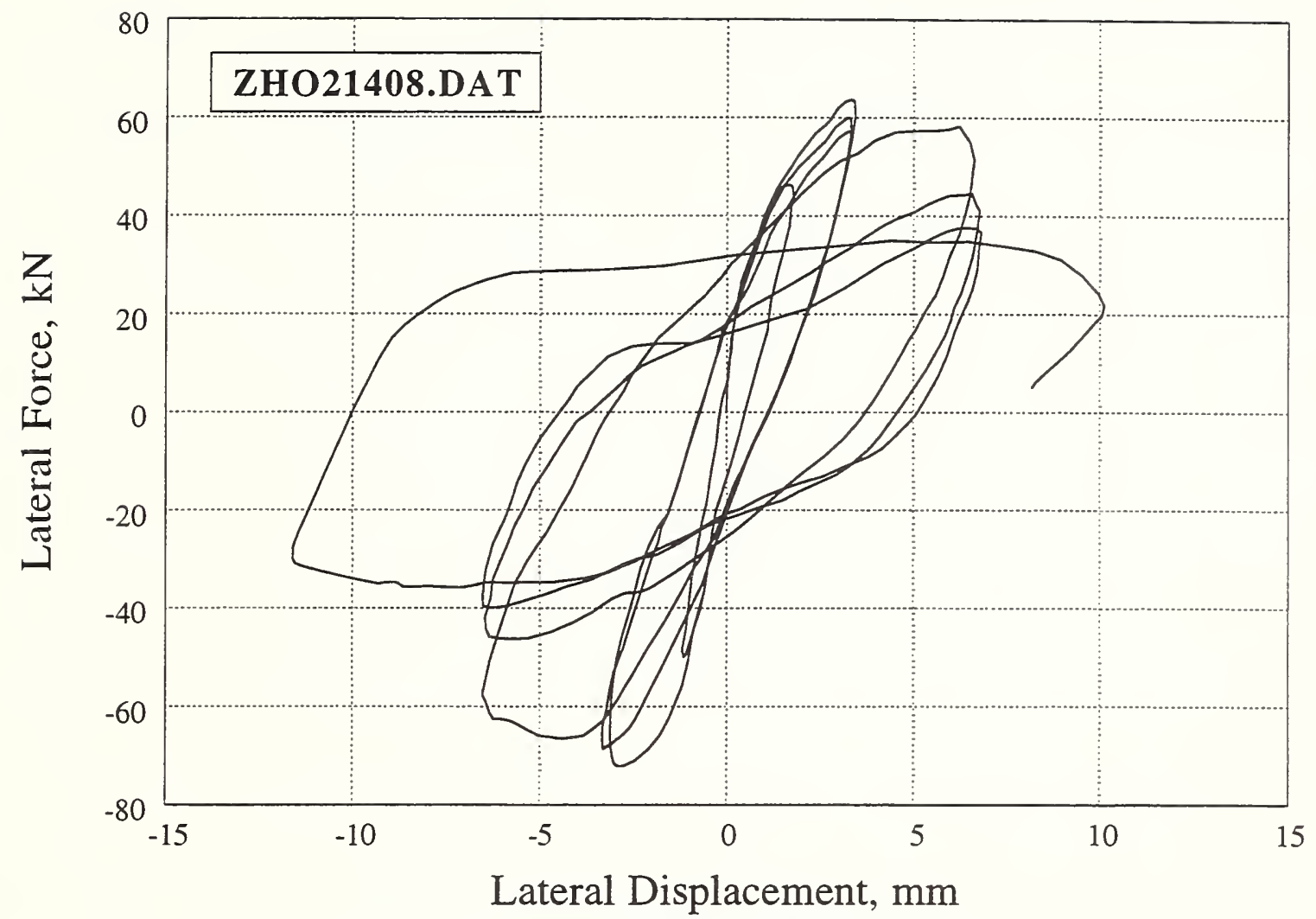

Figure 43. Specimen 214-08 of Zhou et al. 1987

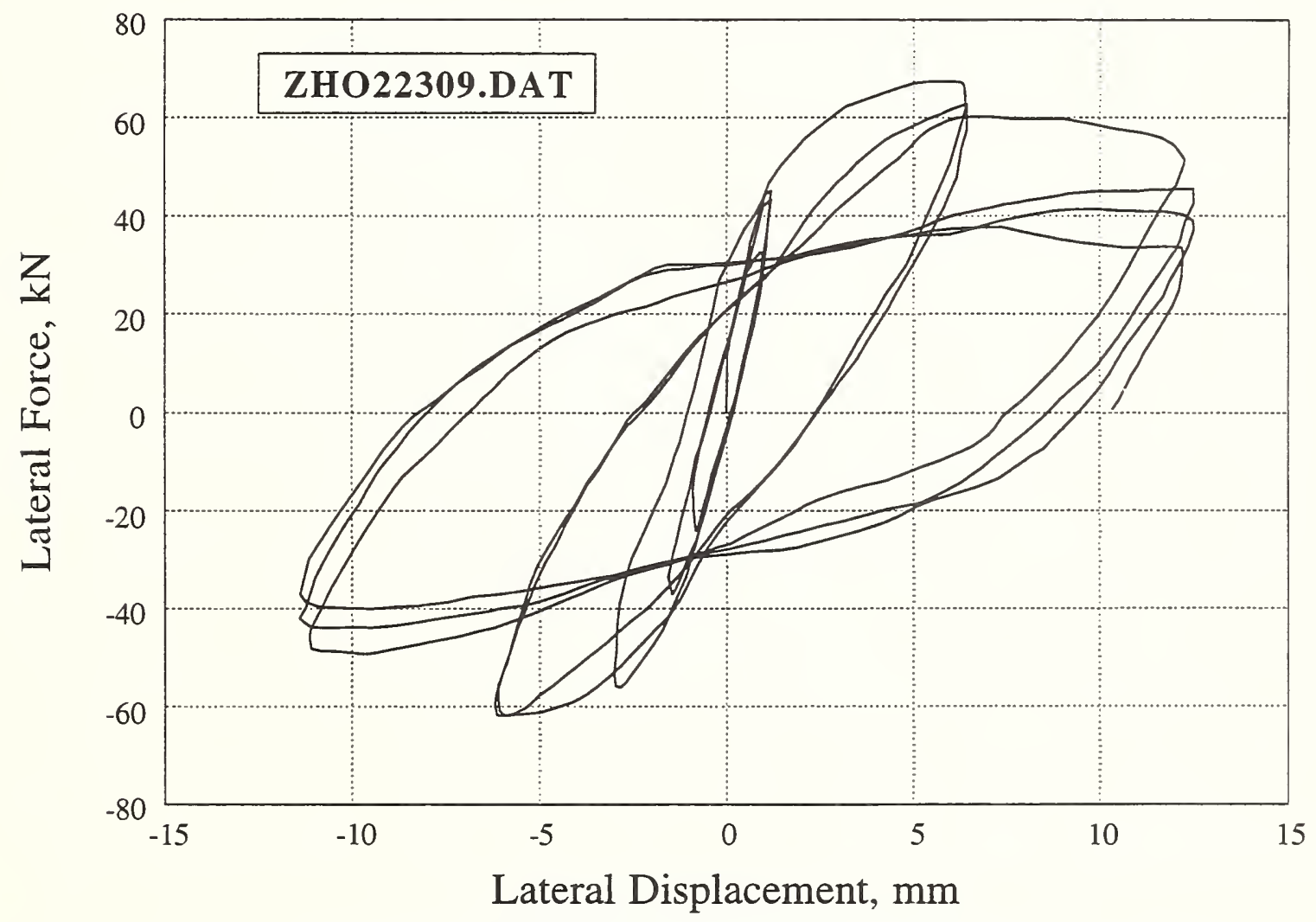

Figure 44. Specimen 223-09 of Zhou et al. 1987 


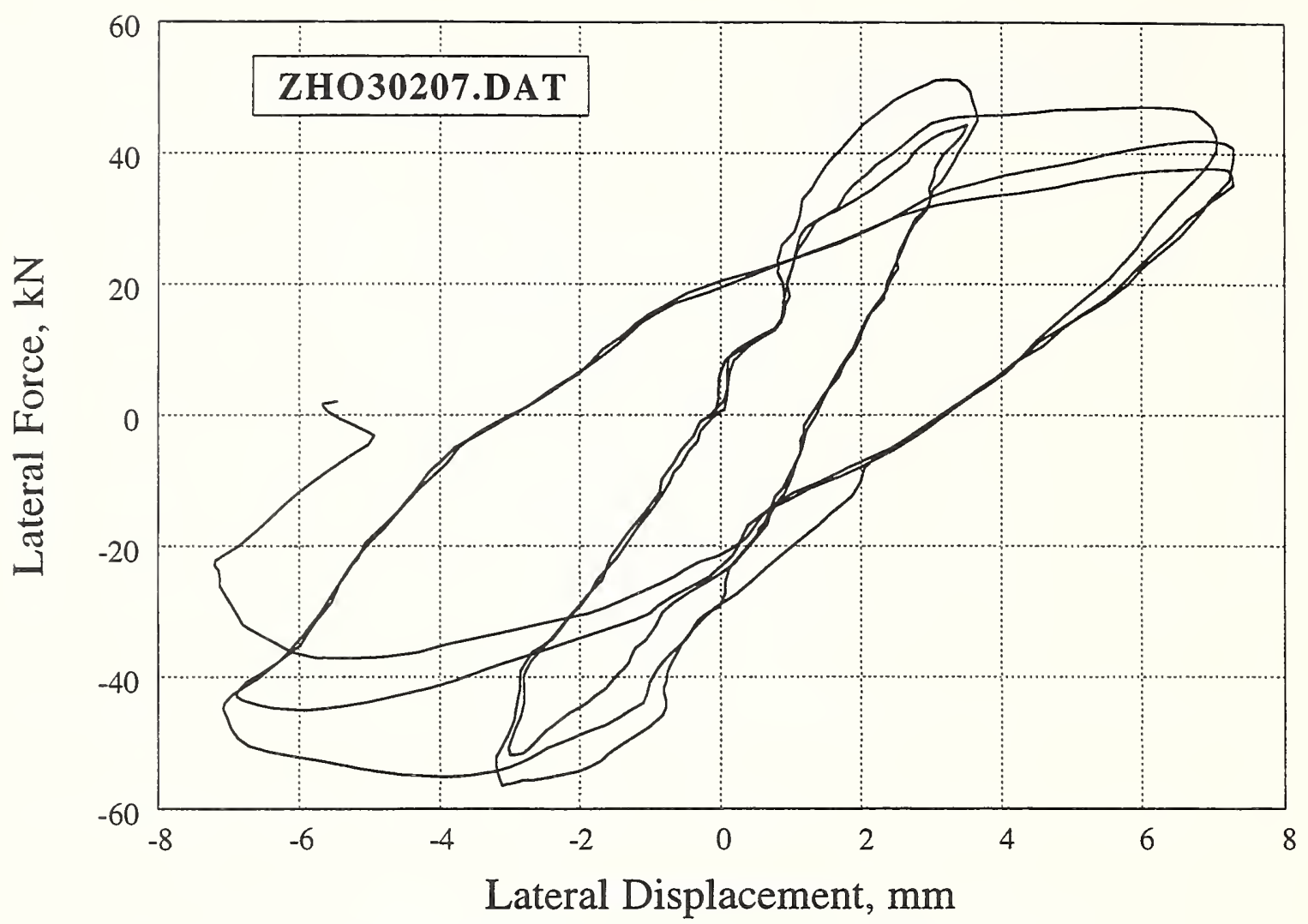

Figure 45. Specimen 302-07 of Zhou et al. 1987

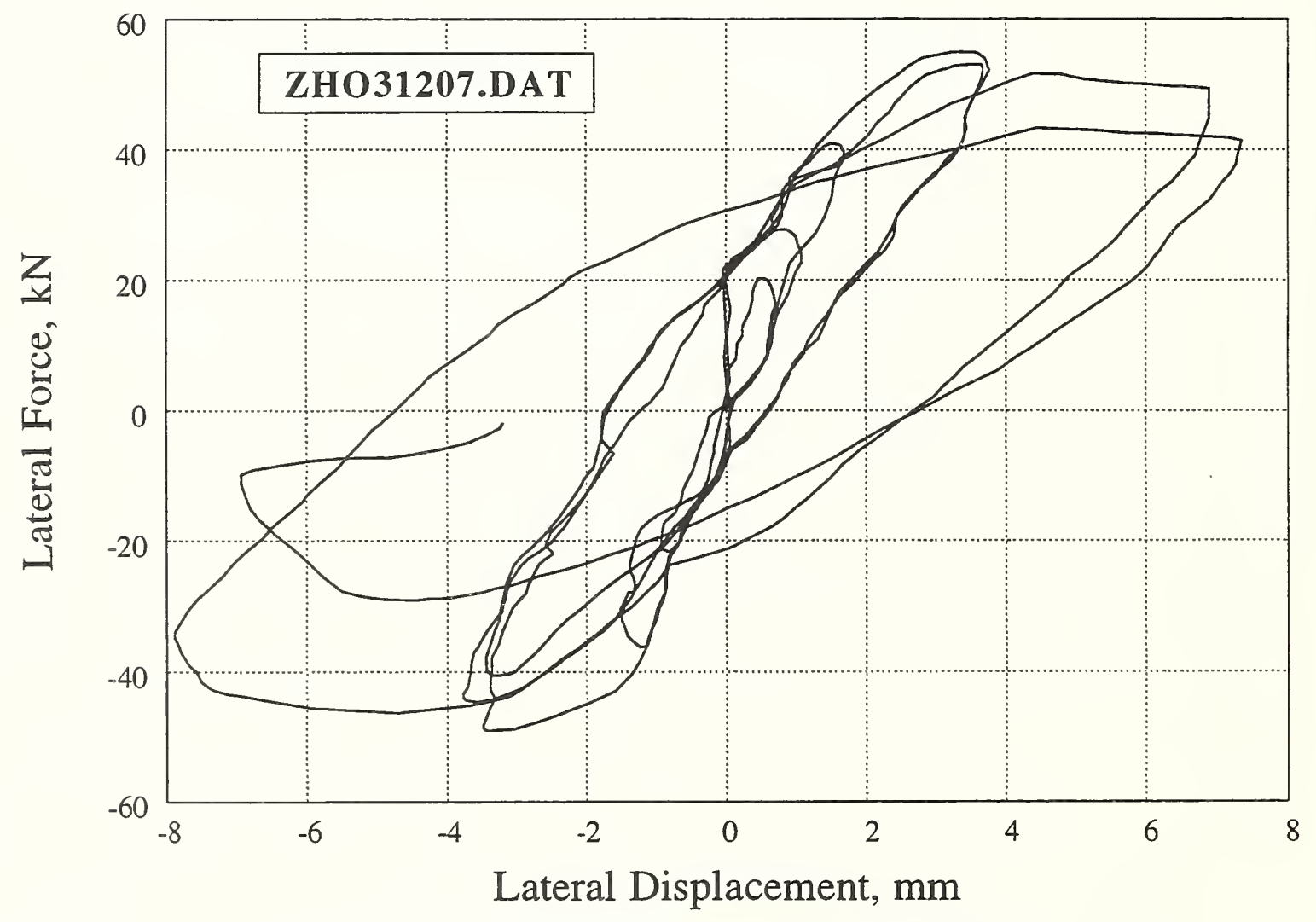

Figure 46. Specimen 312-07 of Zhou et al. 1987 


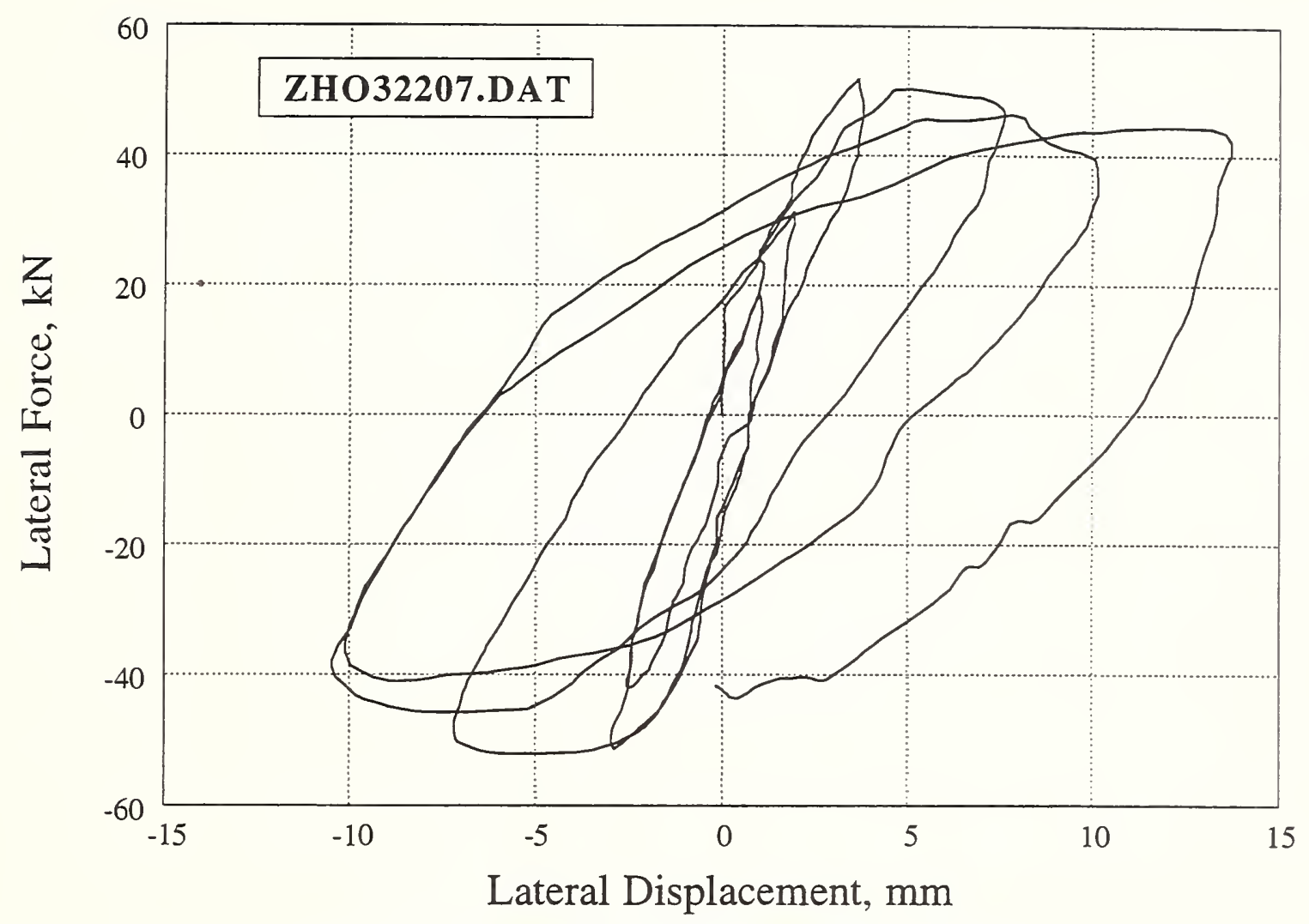

Figure 47. Specimen 322-07 of Zhou et al. 1987

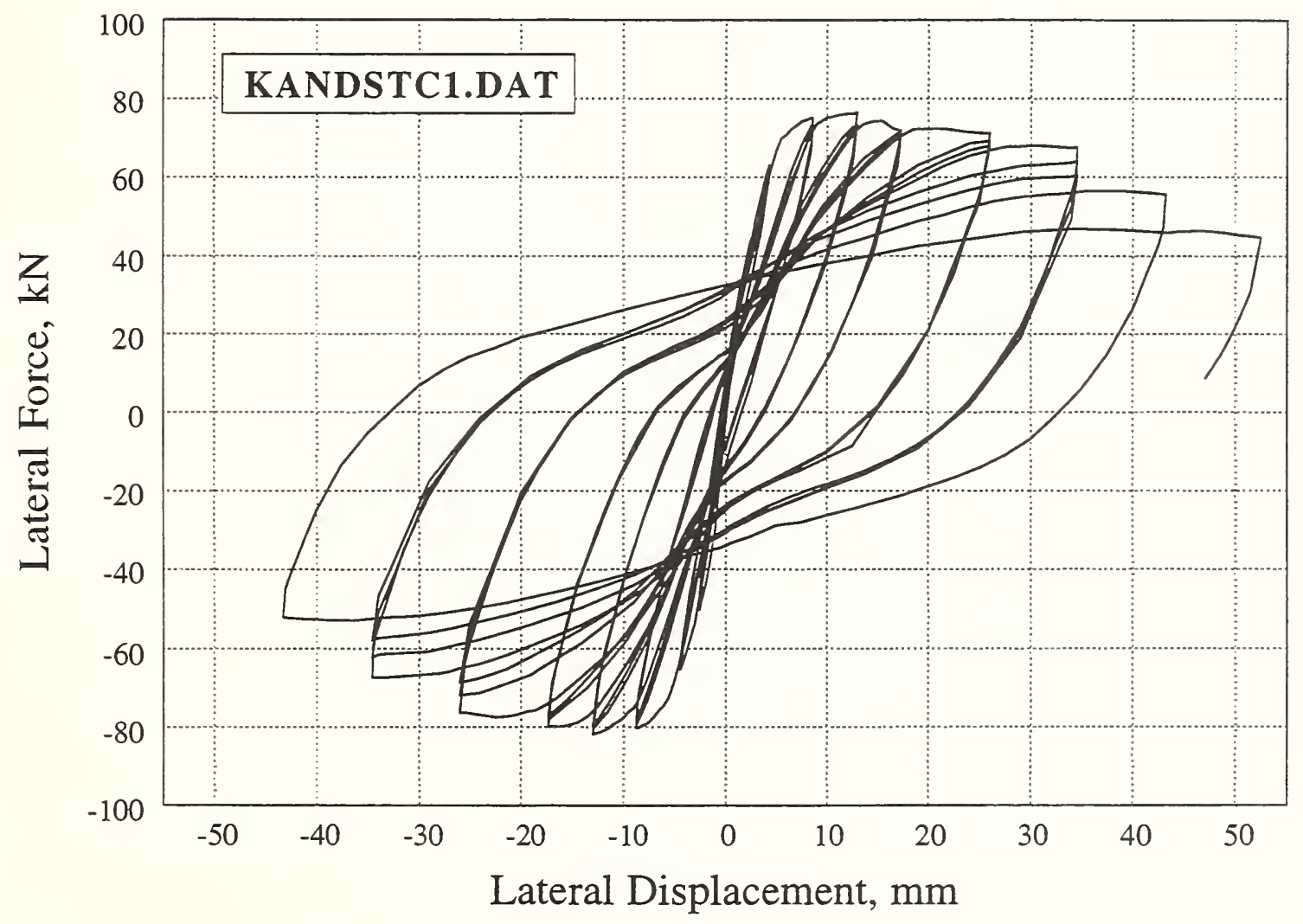

Figure 48. Specimen STC1 of Kanda et al. 1988 


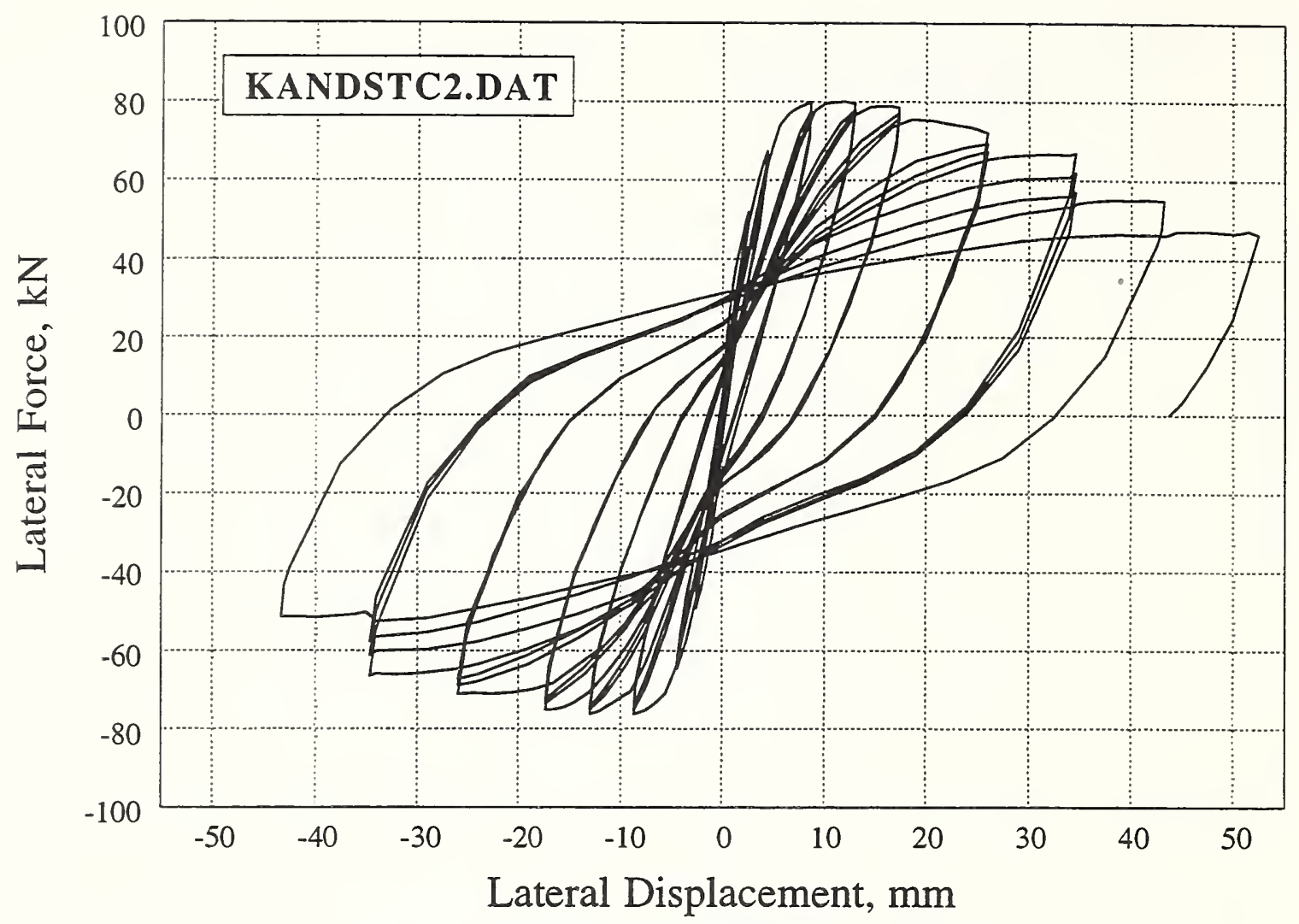

Figure 49. Specimen STC2 of Kanda et al. 1988

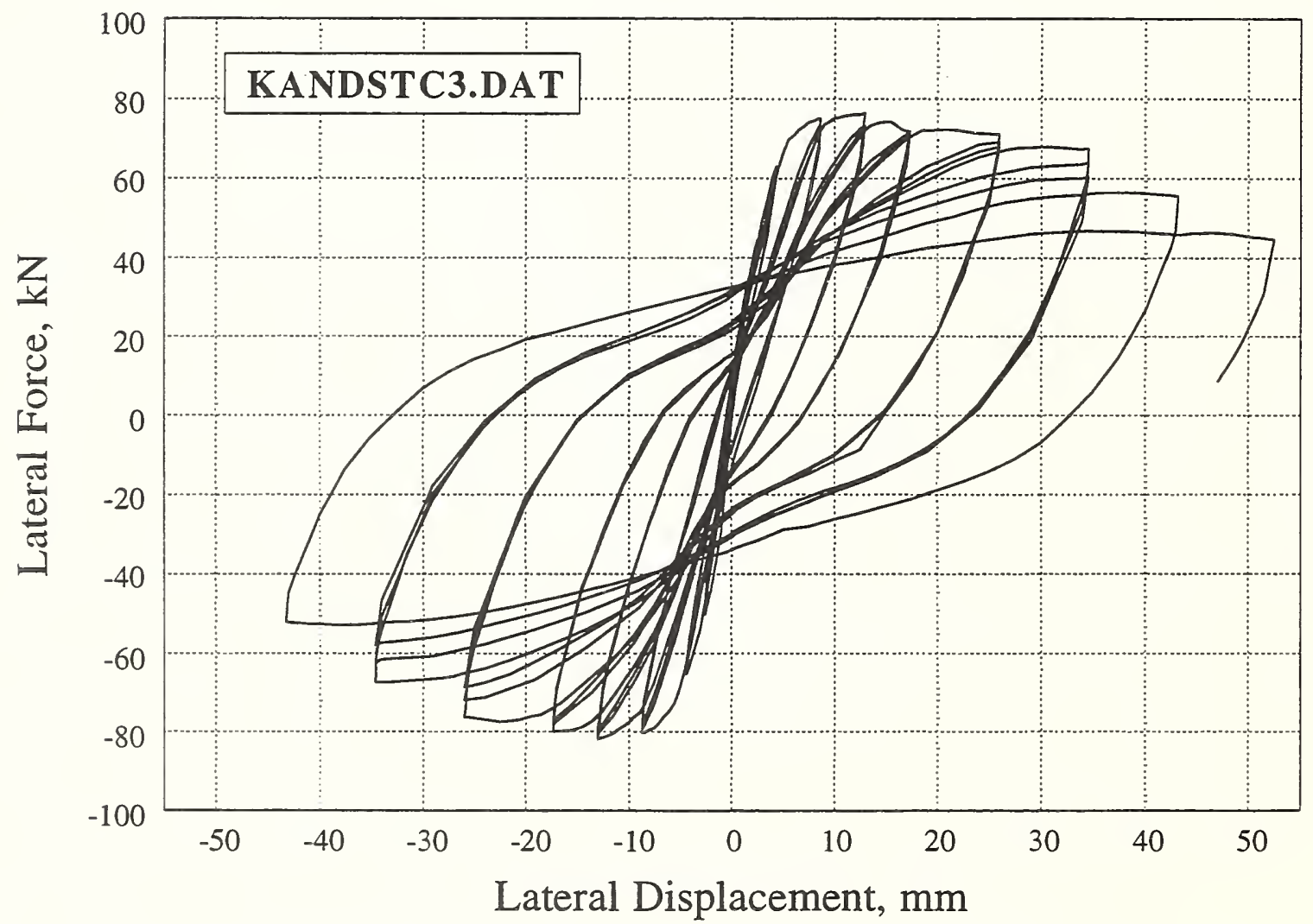

Figure 50. Specimen STC3 of Kanda et al. 1988 


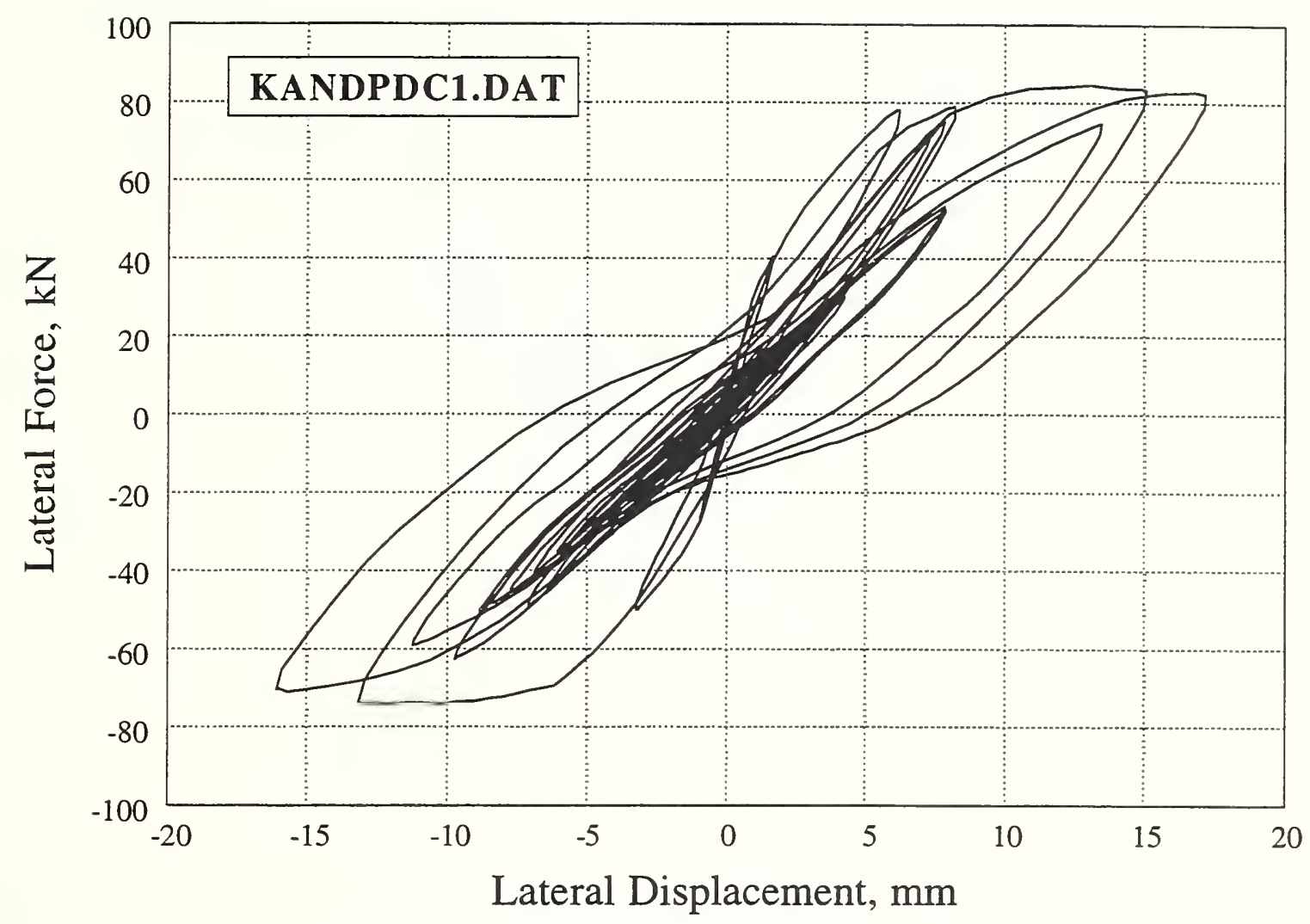

Figure 51. Specimen PDC1 of Kanda et al. 1988

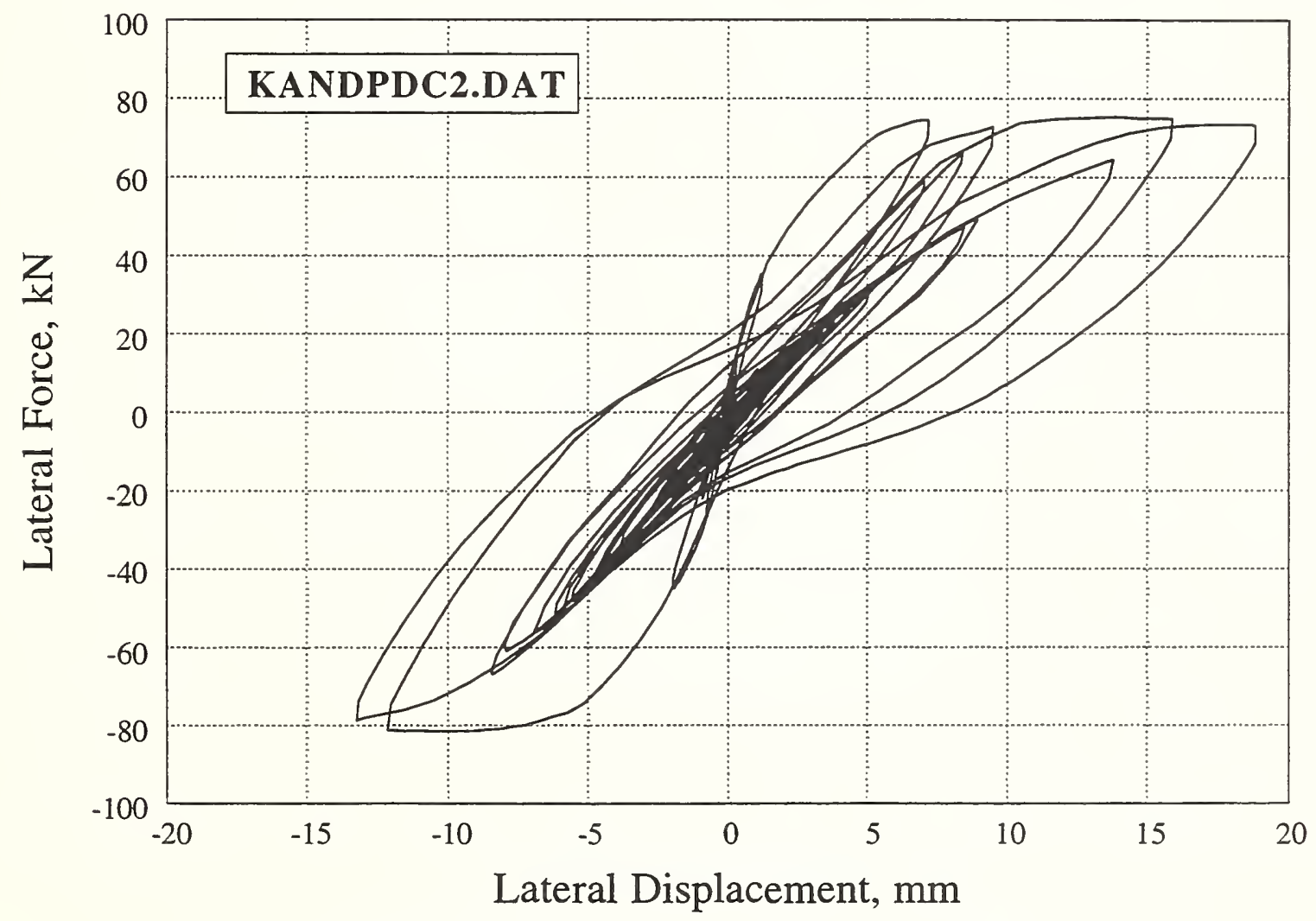

Figure 52. Specimen PDC2 of Kanda et al. 1988 


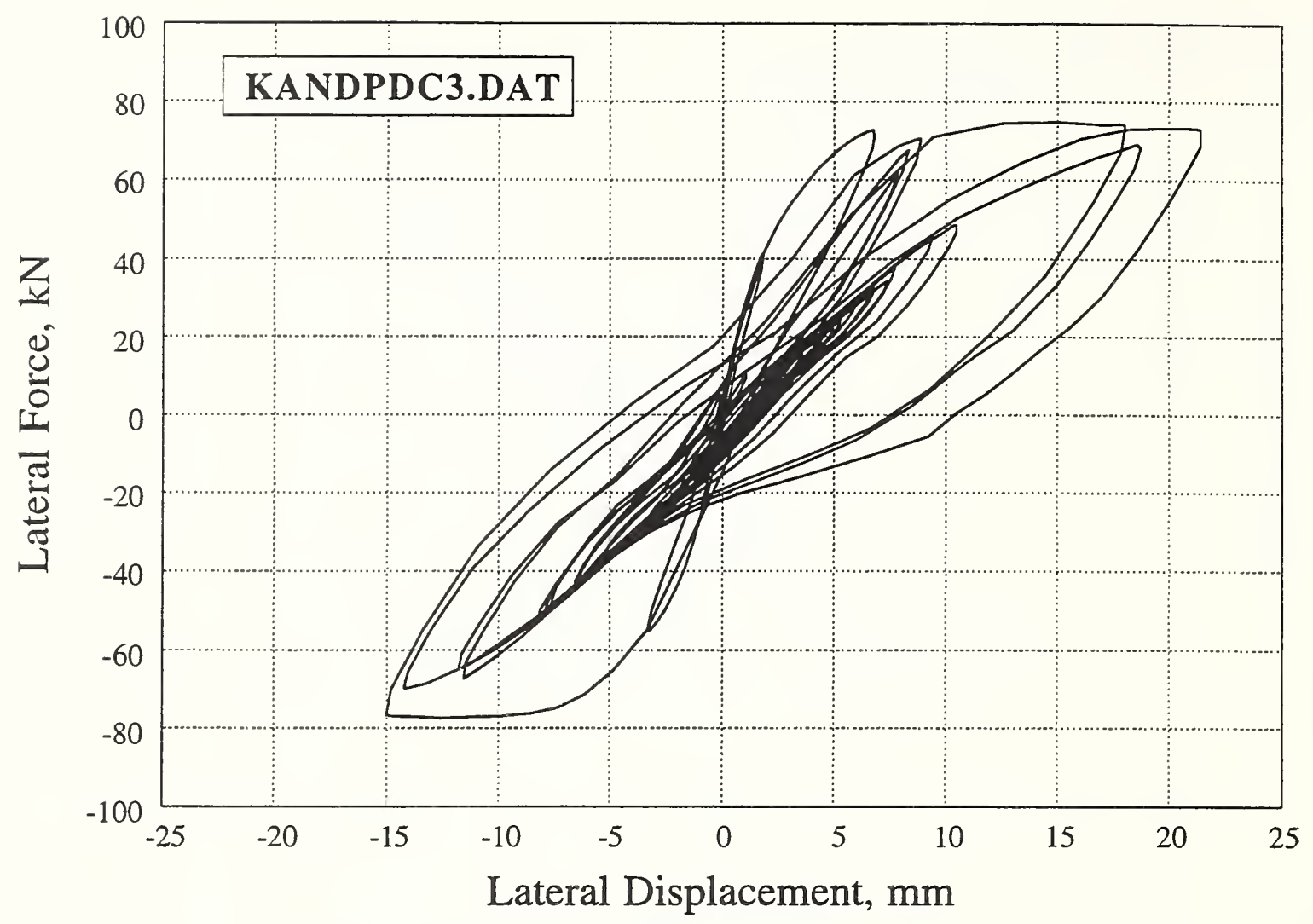

Figure 53. Specimen PDC3 of Kanda et al. 1988

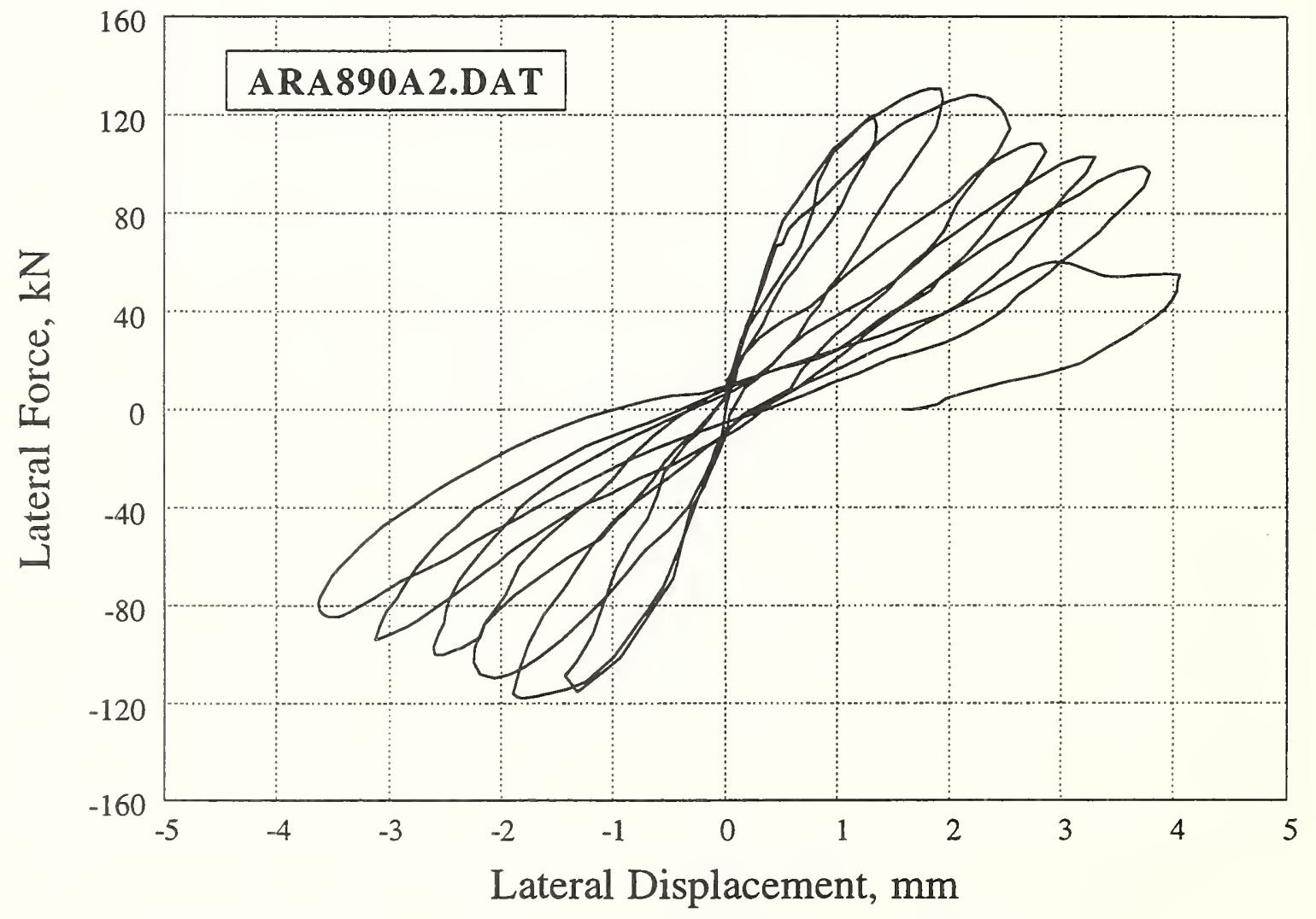

Figure 54. Specimen OA2 of Arakawa 1989 


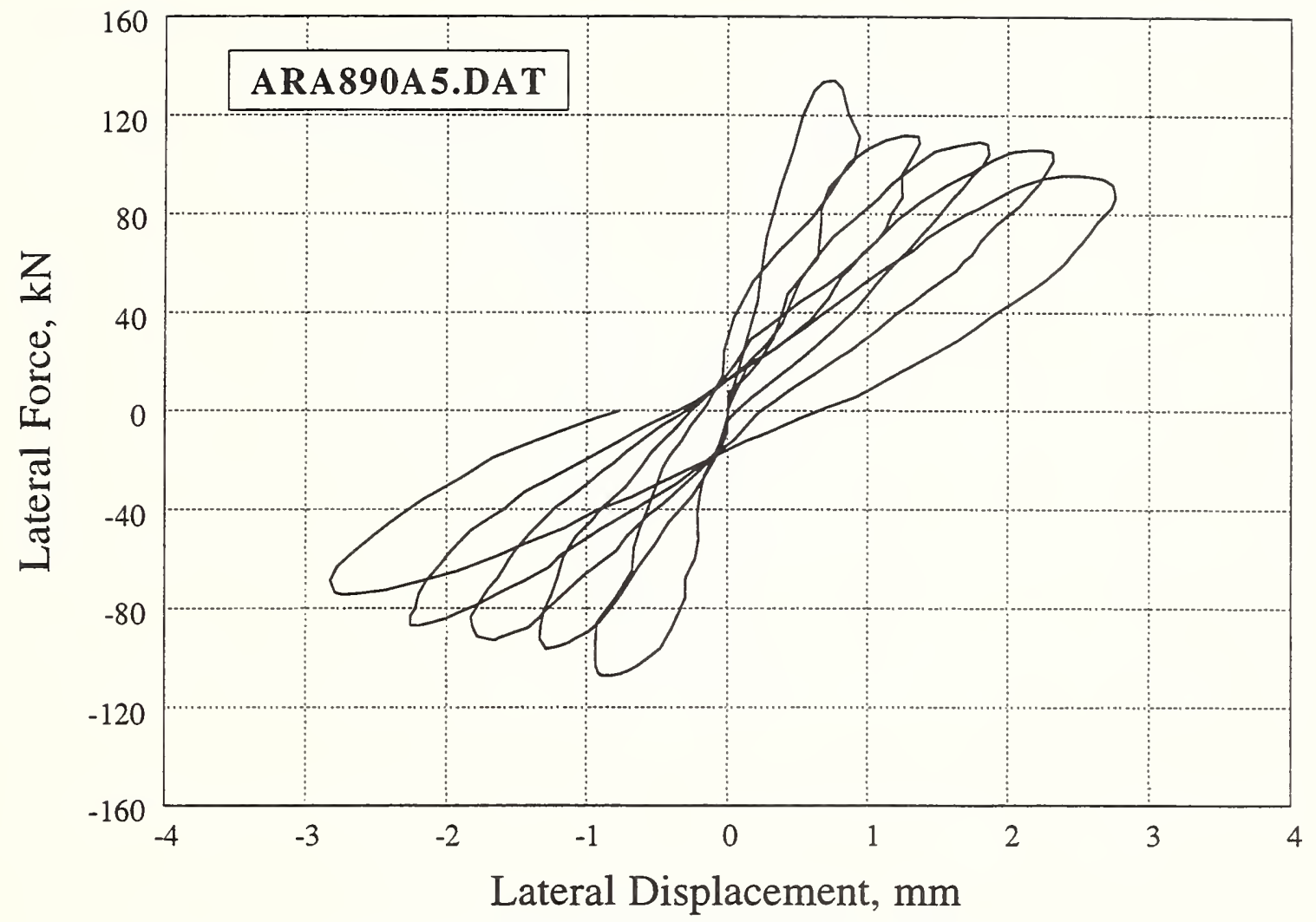

Figure 55. Specimen OA5 of Arakawa 1989

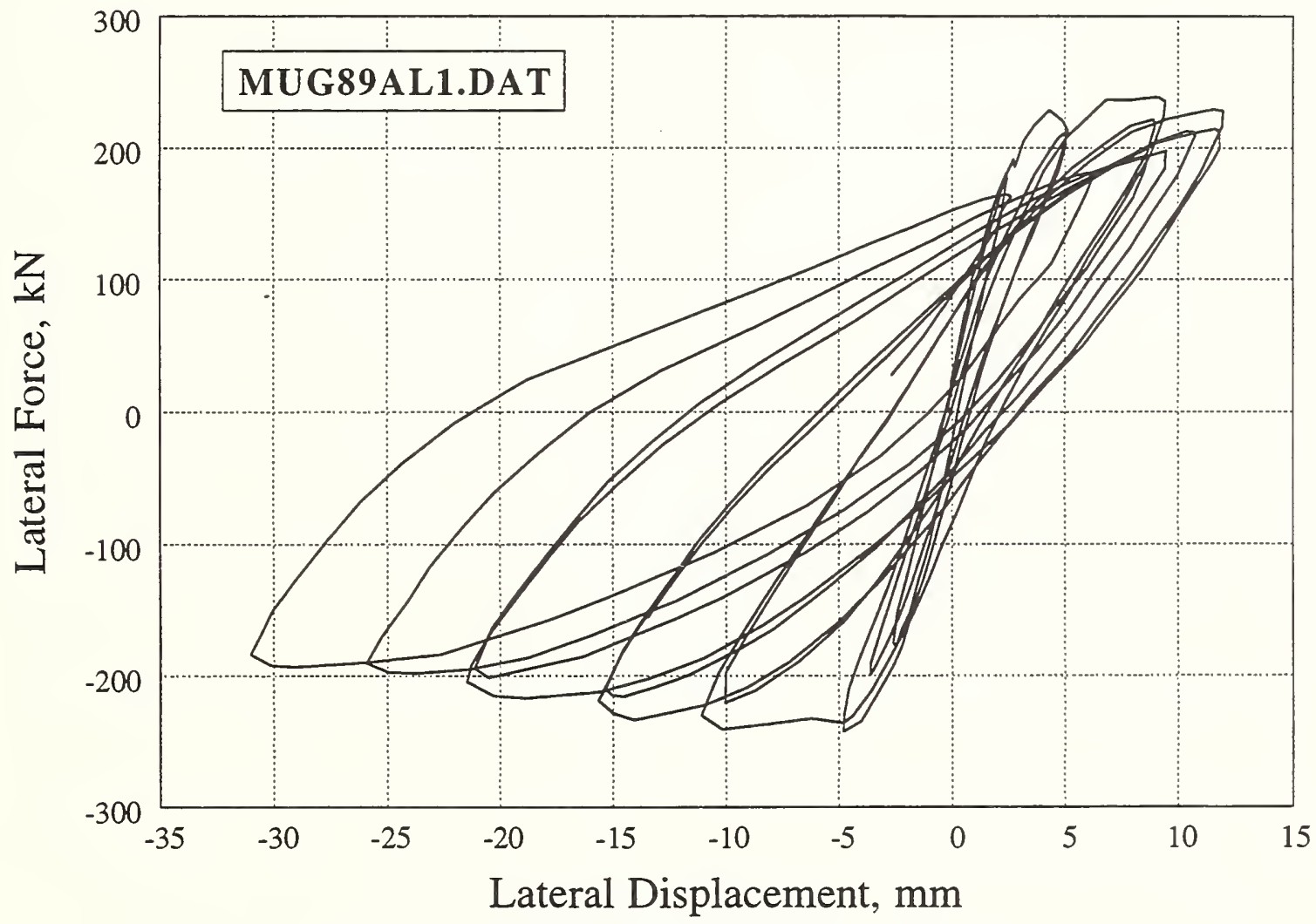

Figure 56. Specimen AL-1 of Muguruma 1989 


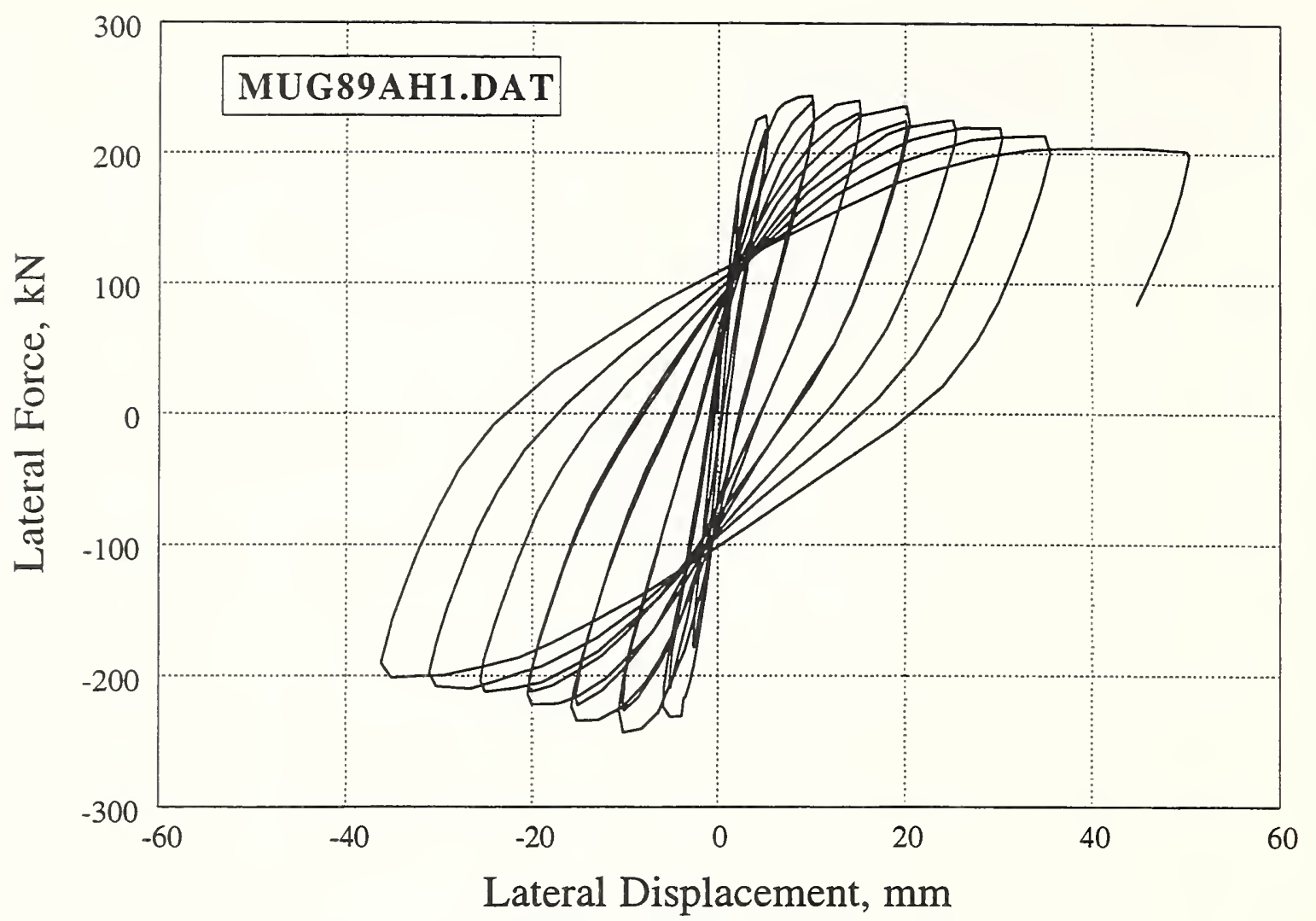

Figure 57. Specimen AH-1 of Muguruma 1989

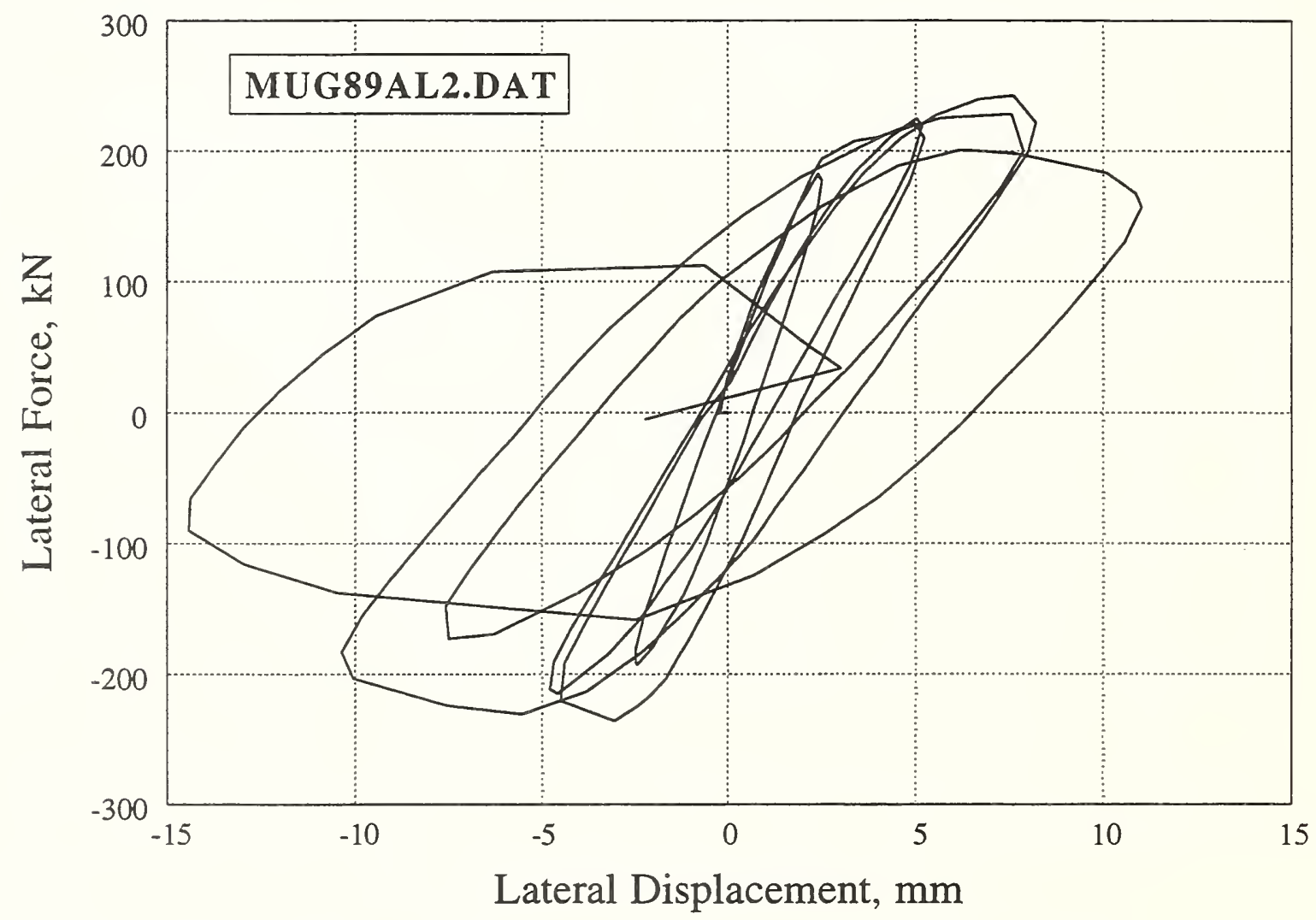

Figure 58. Specimen AL-2 of Muguruma 1989 


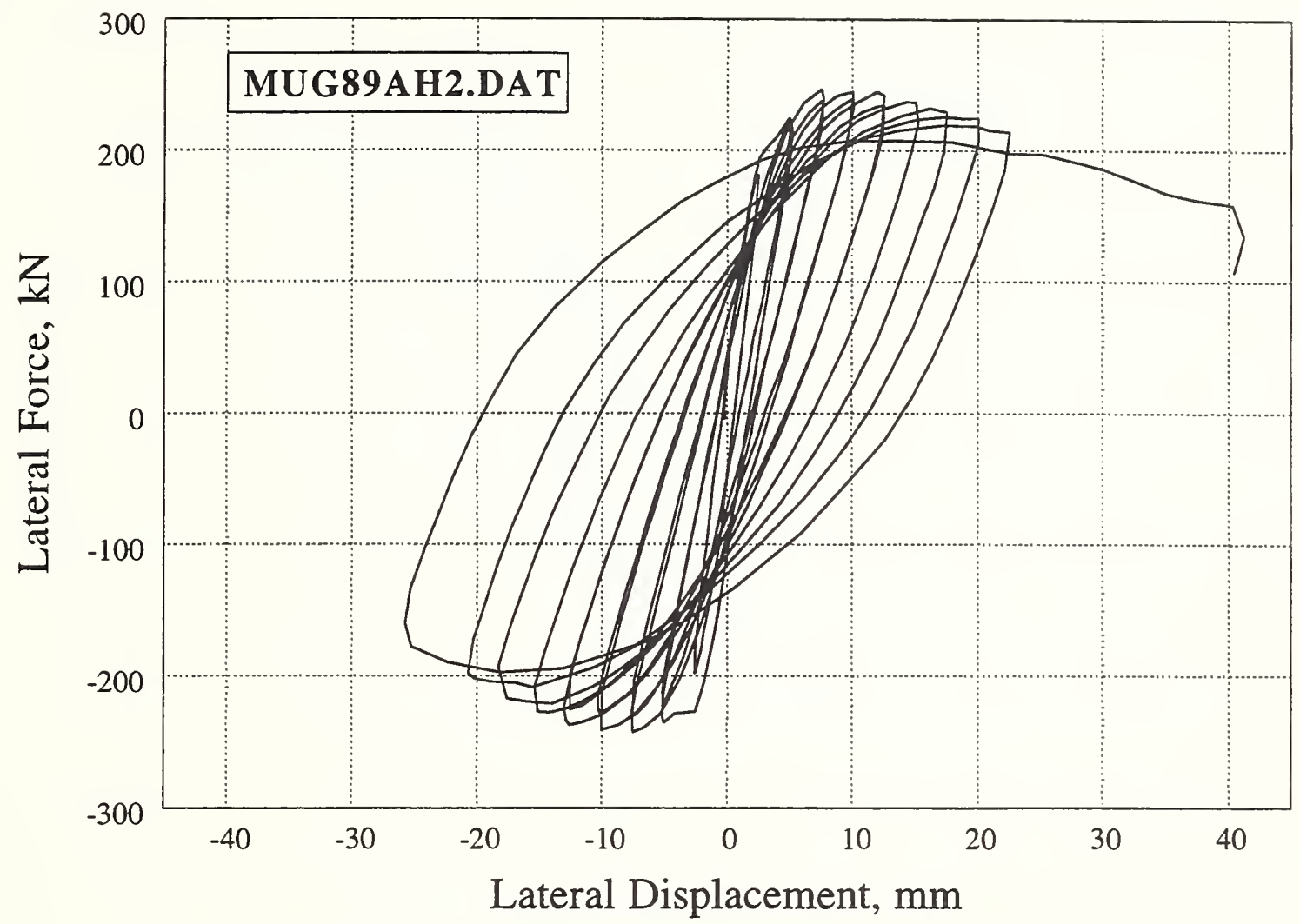

Figure 59. Specimen AH-2 of Muguruma 1989

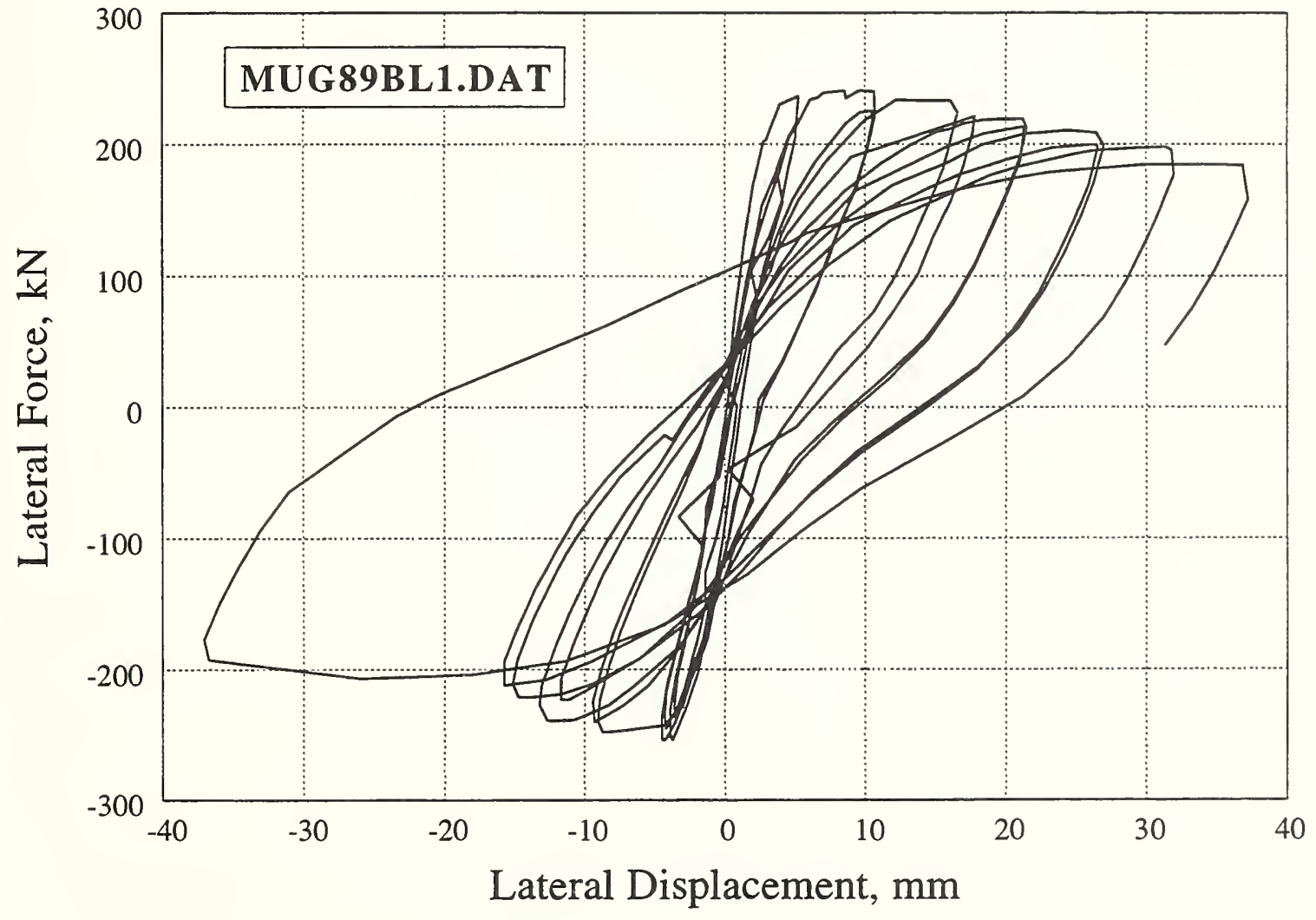

Figure 60. Specimen BL-1 of Muguruma 1989 


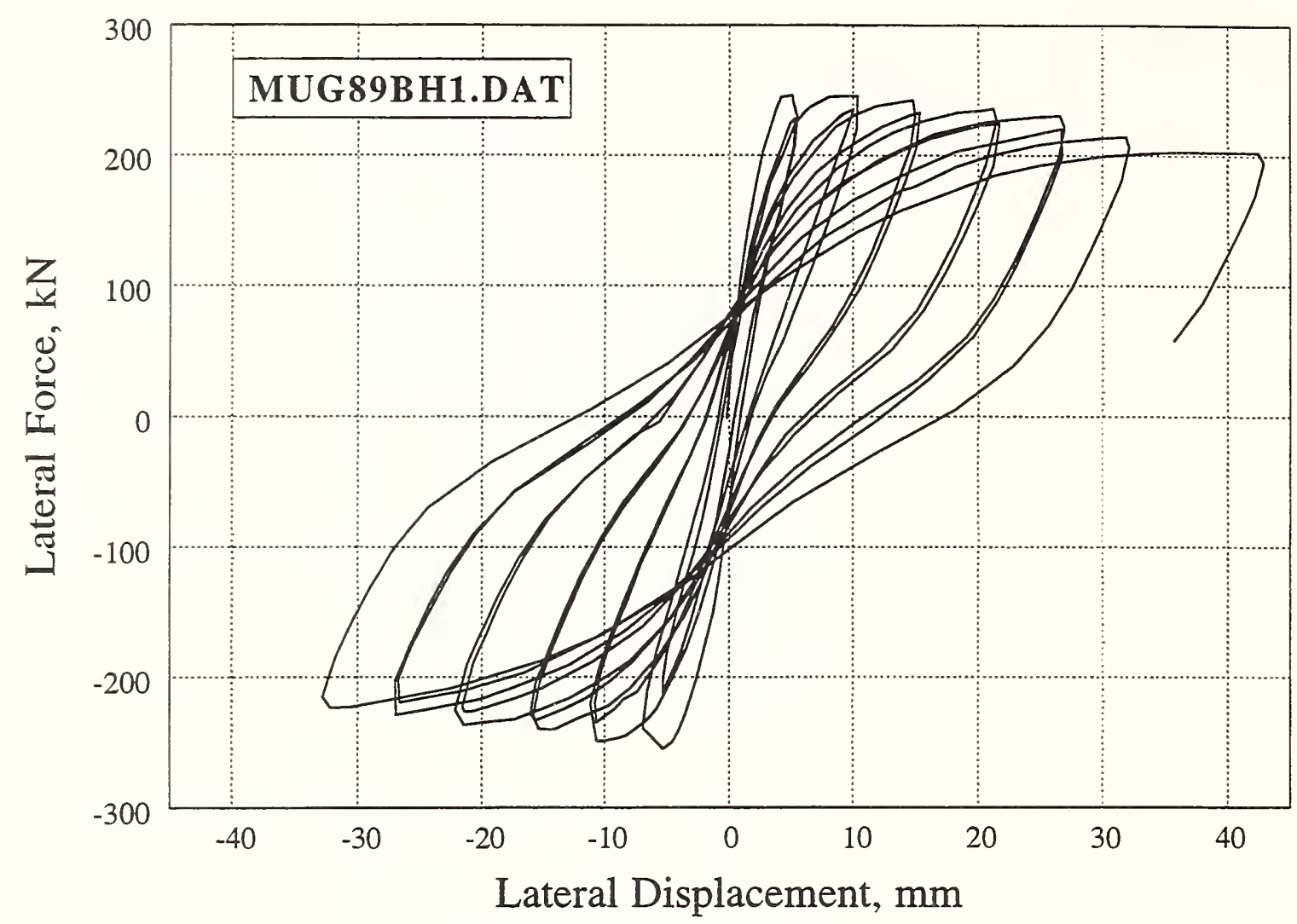

Figure 61. Specimen BH-1 of Muguruma 1989

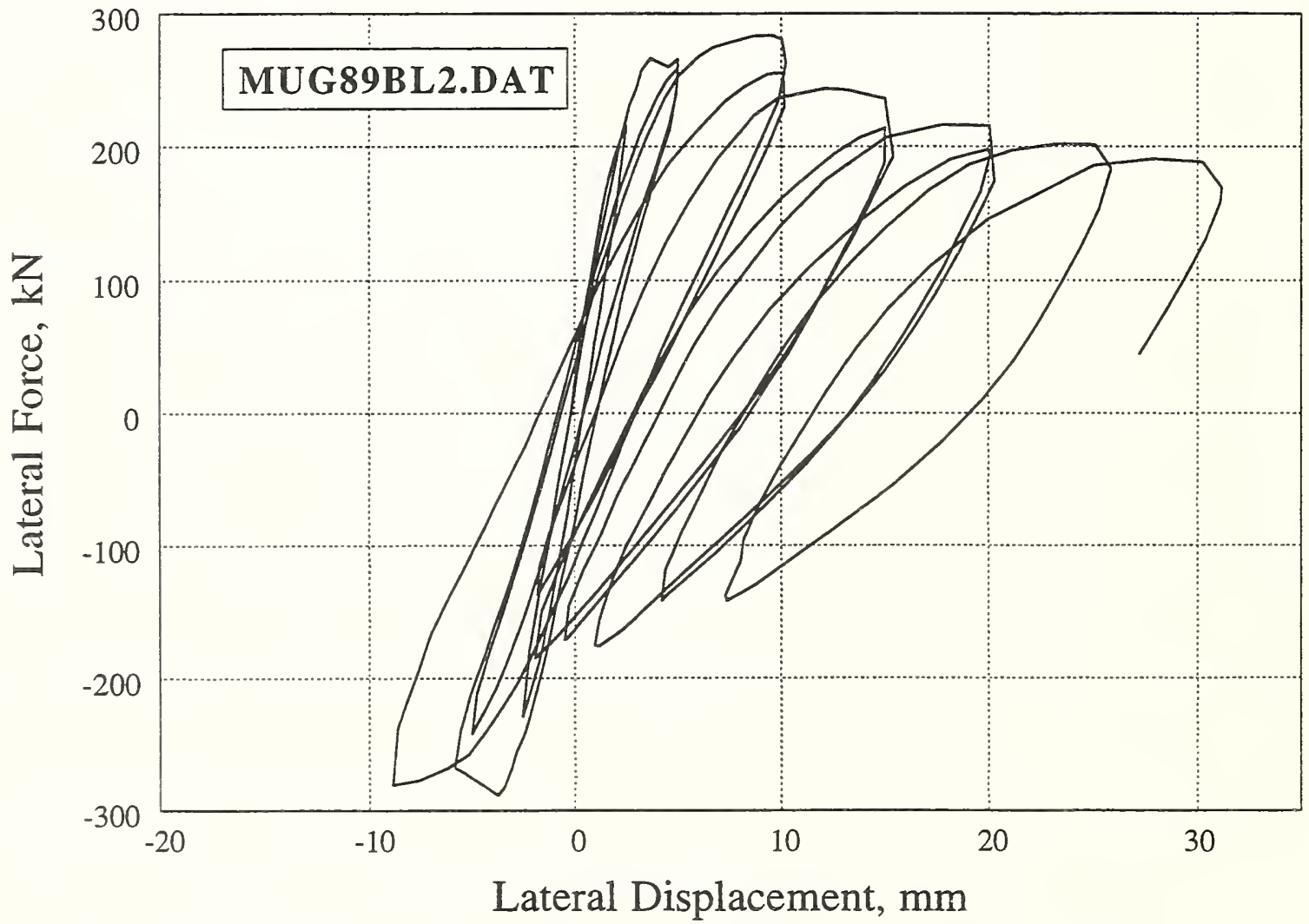

Figure 62. Specimen BL-2 of Muguruma 1989 


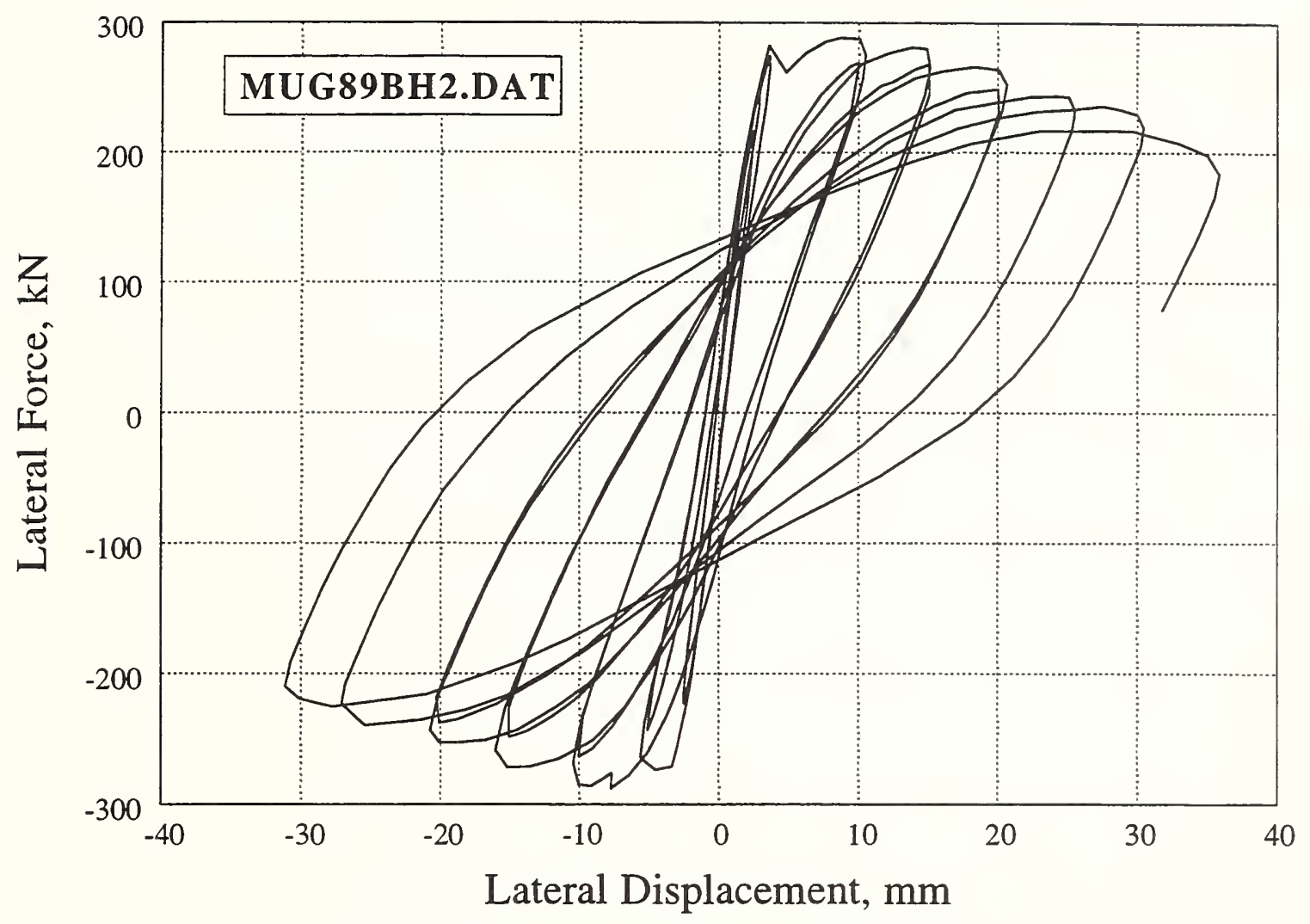

Figure 63. Specimen BH-2 of Muguruma 1989

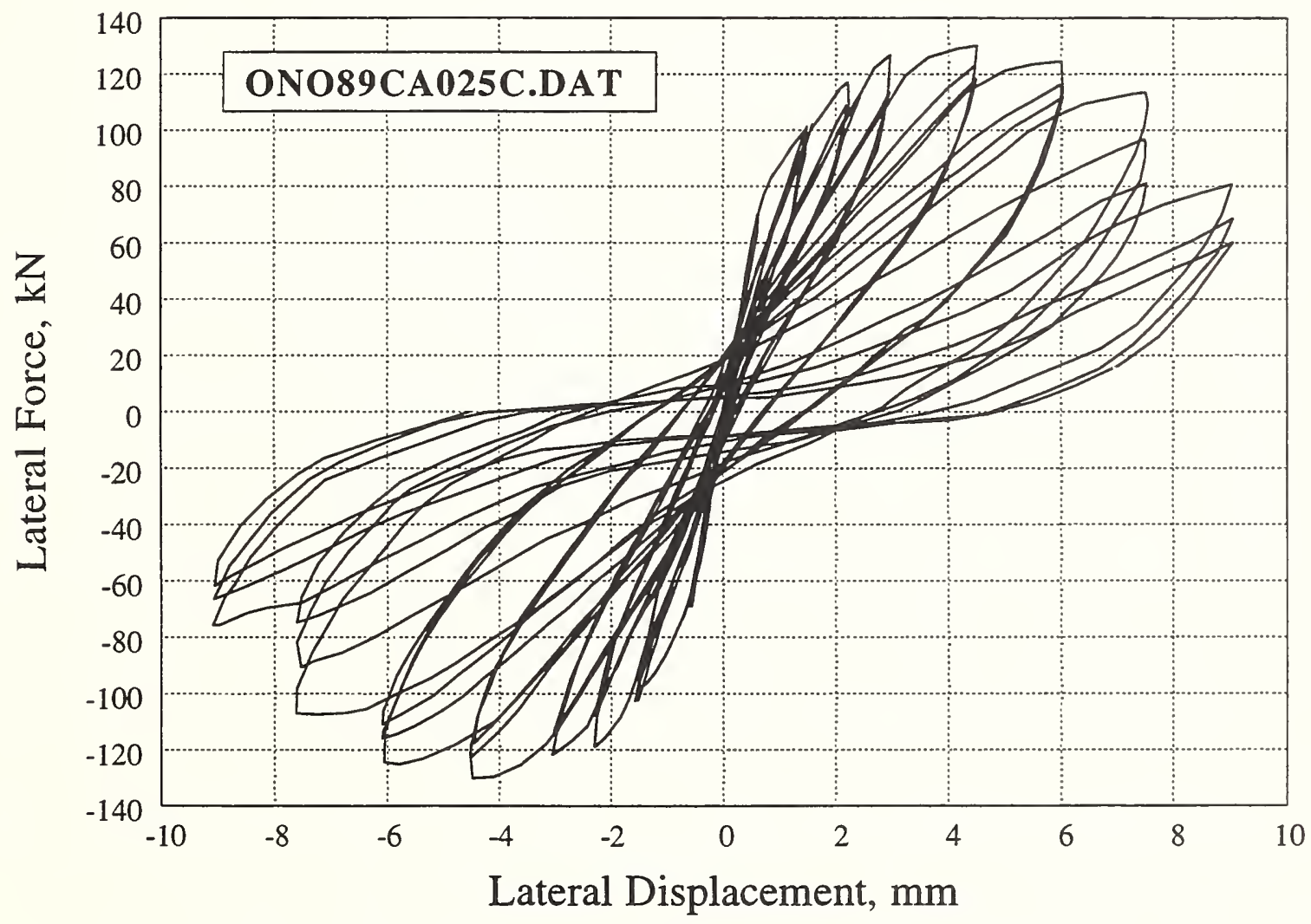

Figure 64. Specimen CA025C of Ono 1989 


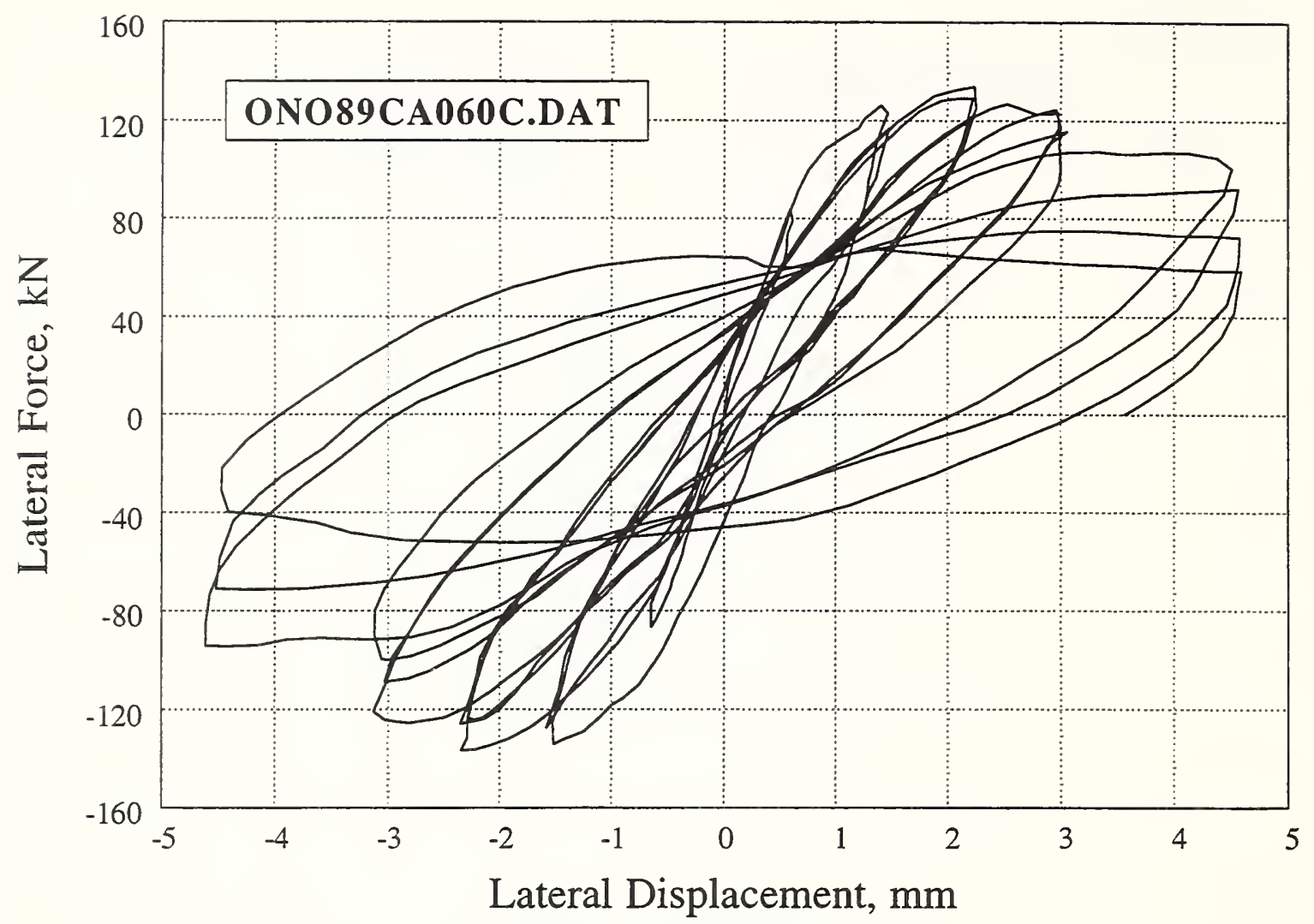

Figure 65. Specimen CA060C of Ono 1989

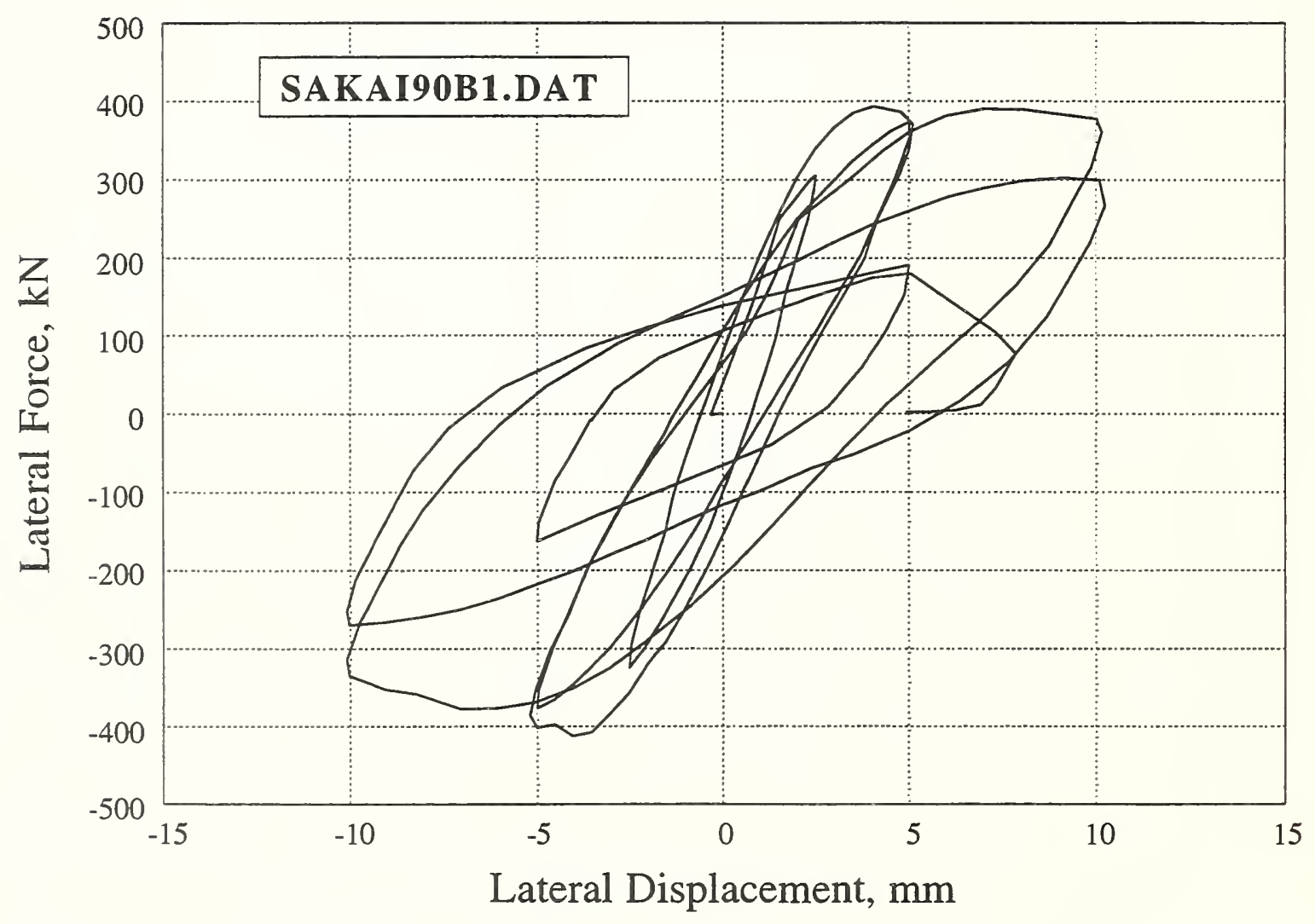

Figure 66. Specimen B1 of Sakai 1990 


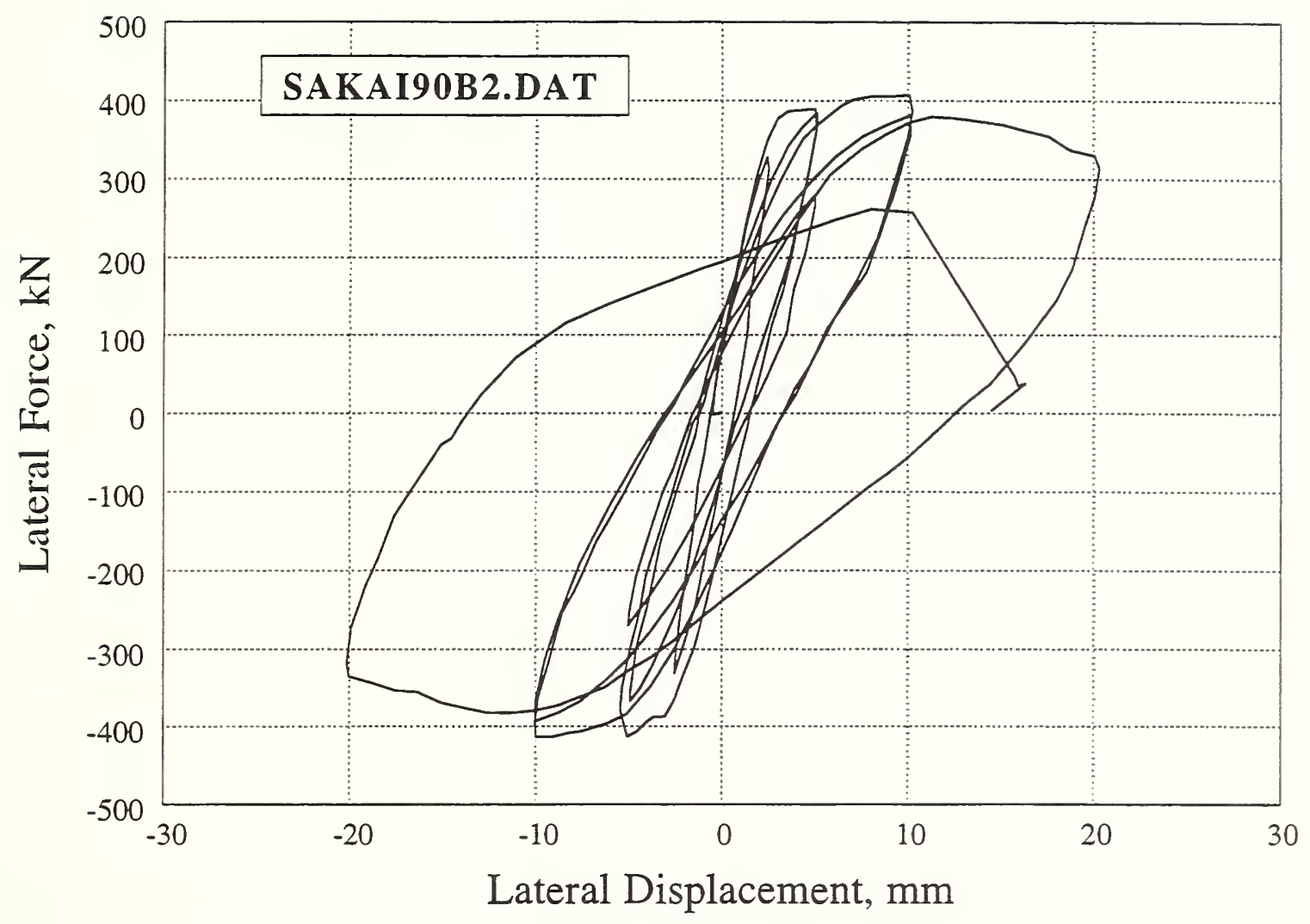

Figure 67. Specimen B2 of Sakai 1990

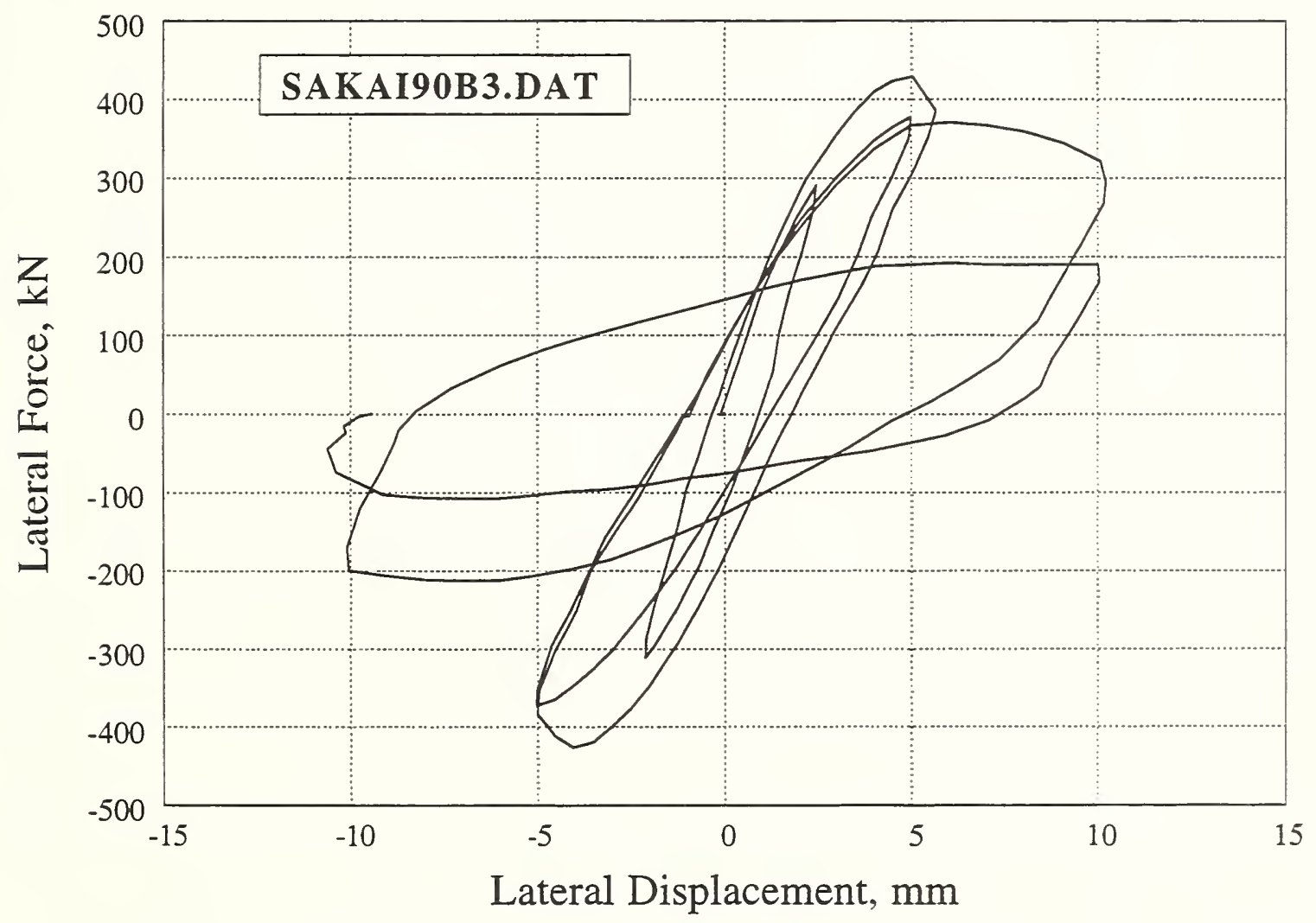

Figure 68. Specimen B3 of Sakai 1990 


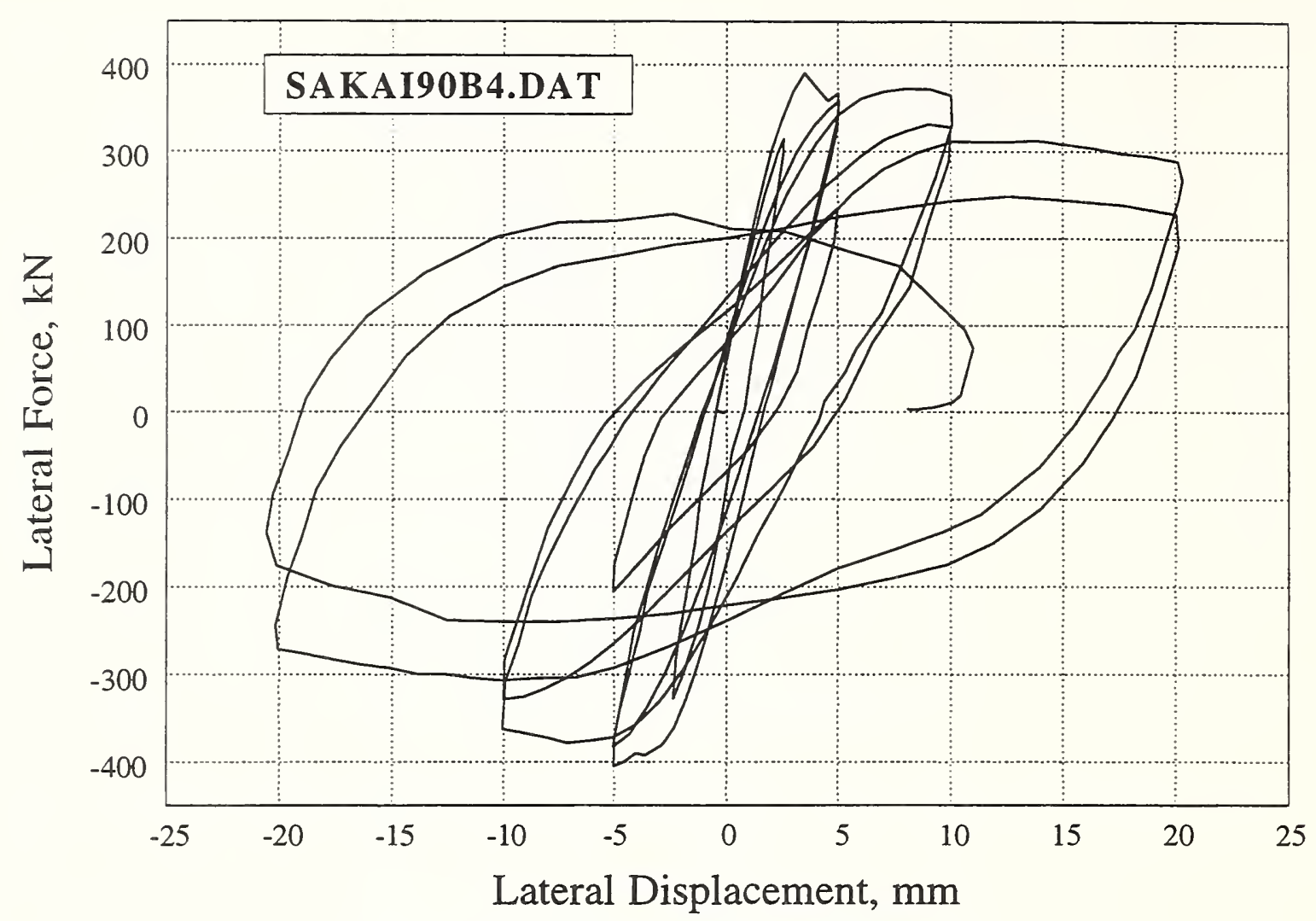

Figure 69. Specimen B4 of Sakai 1990

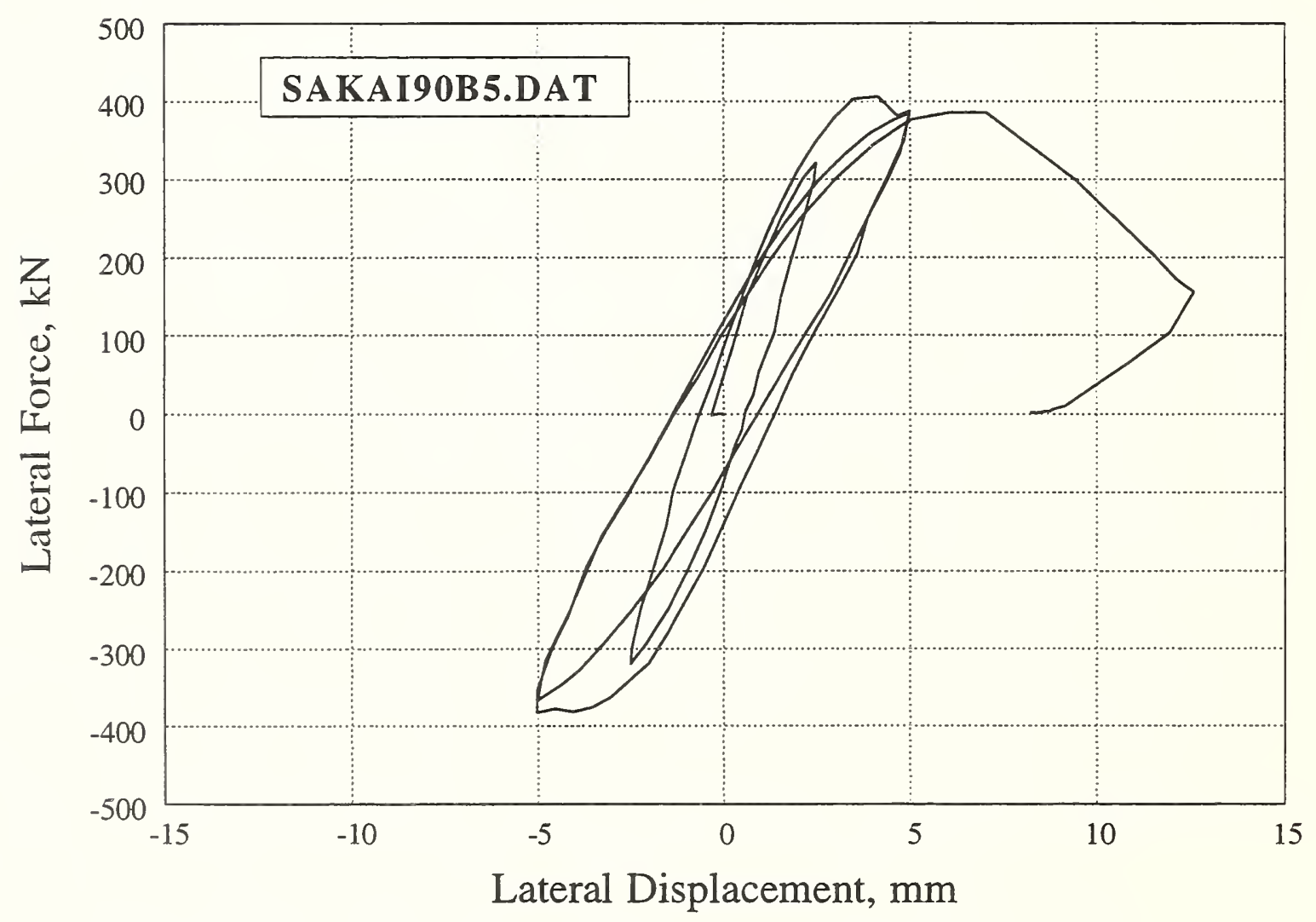

Figure 70. Specimen B5 of Sakai 1990 


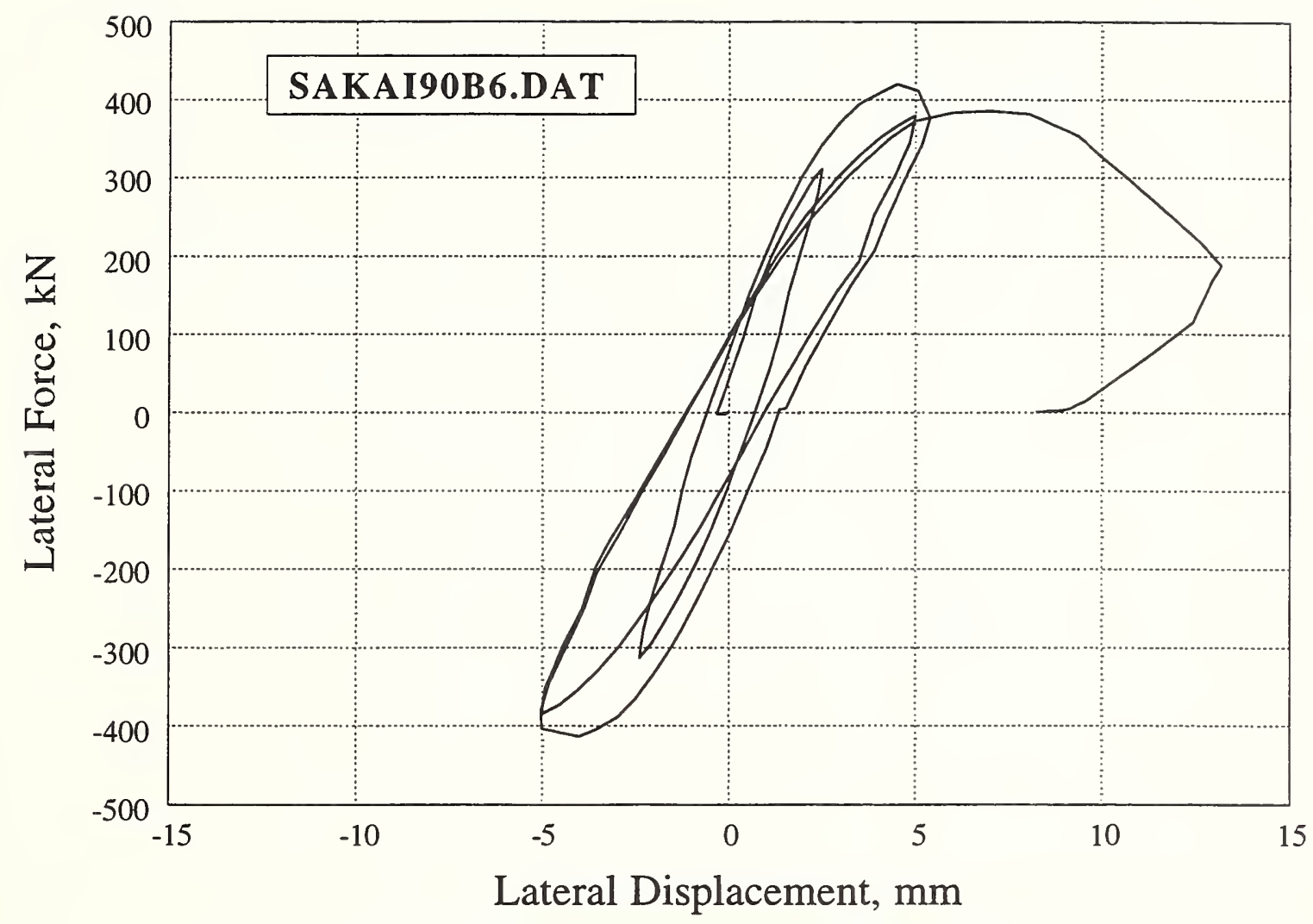

Figure 71. Specimen B6 of Sakai 1990

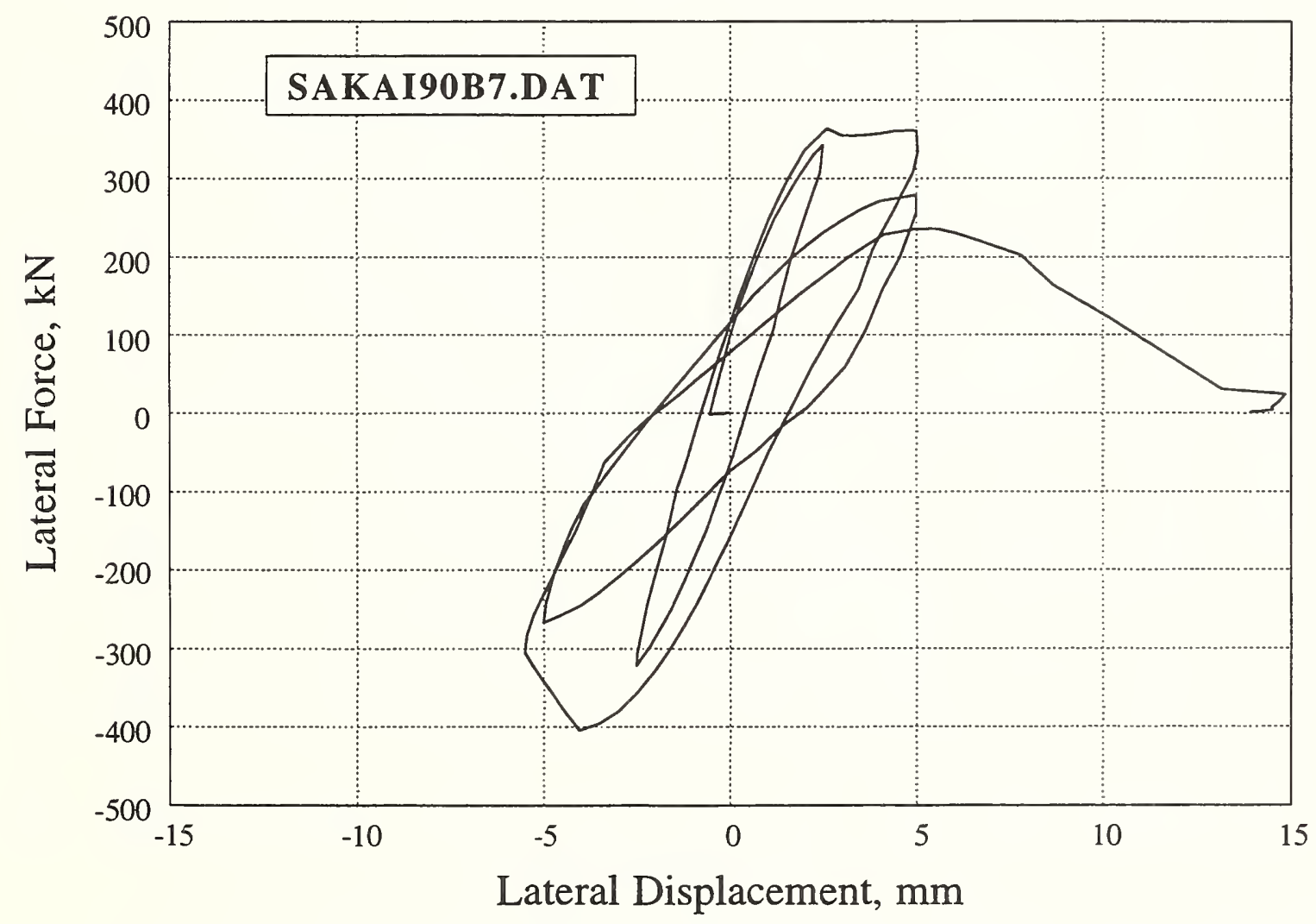

Figure 72. Specimen B7 of Sakai 1990 


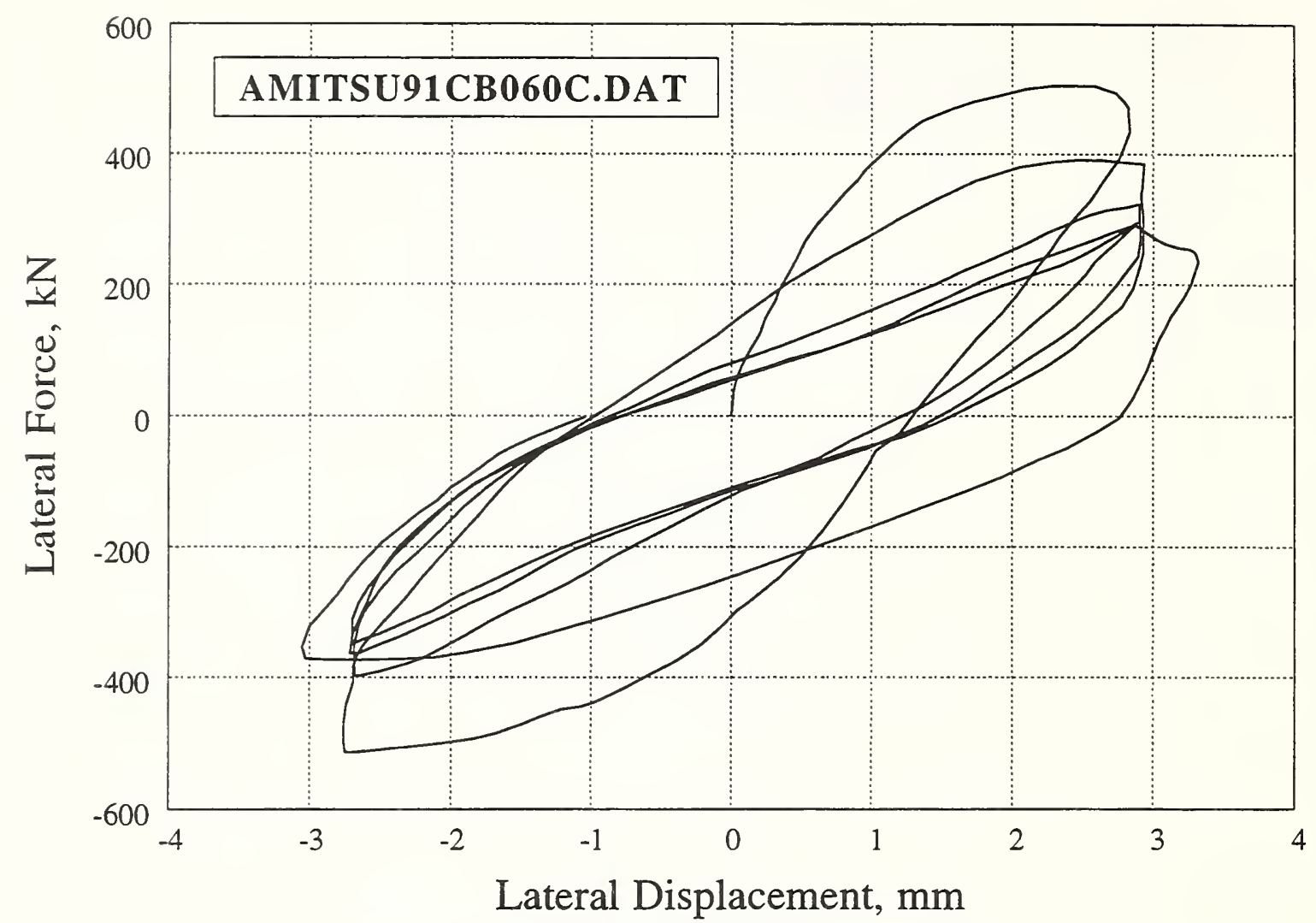

Figure 73. Specimen CB060C of Amitsu 1991

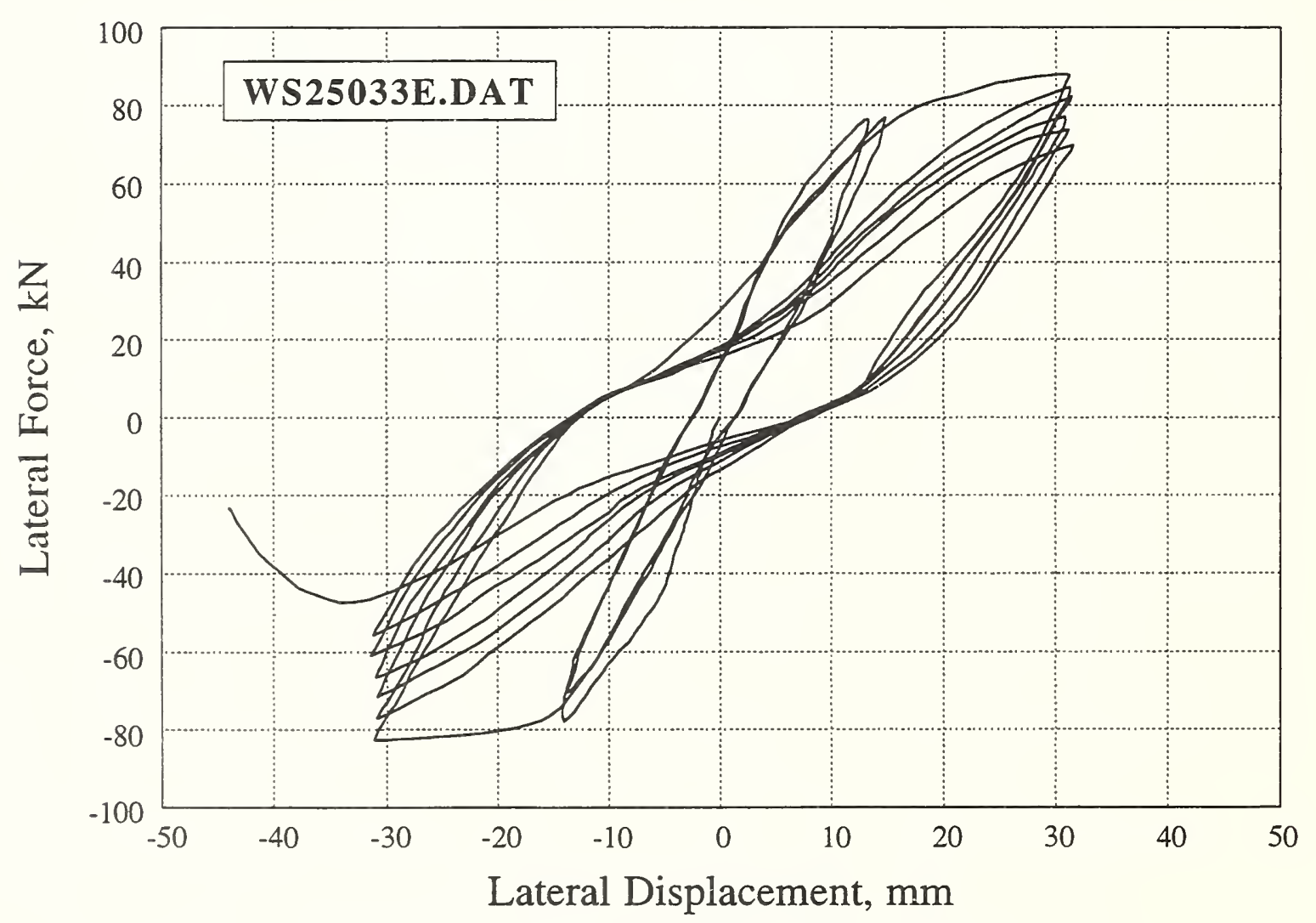

Figure 74. Specimen WS25033E of Wight 1973 


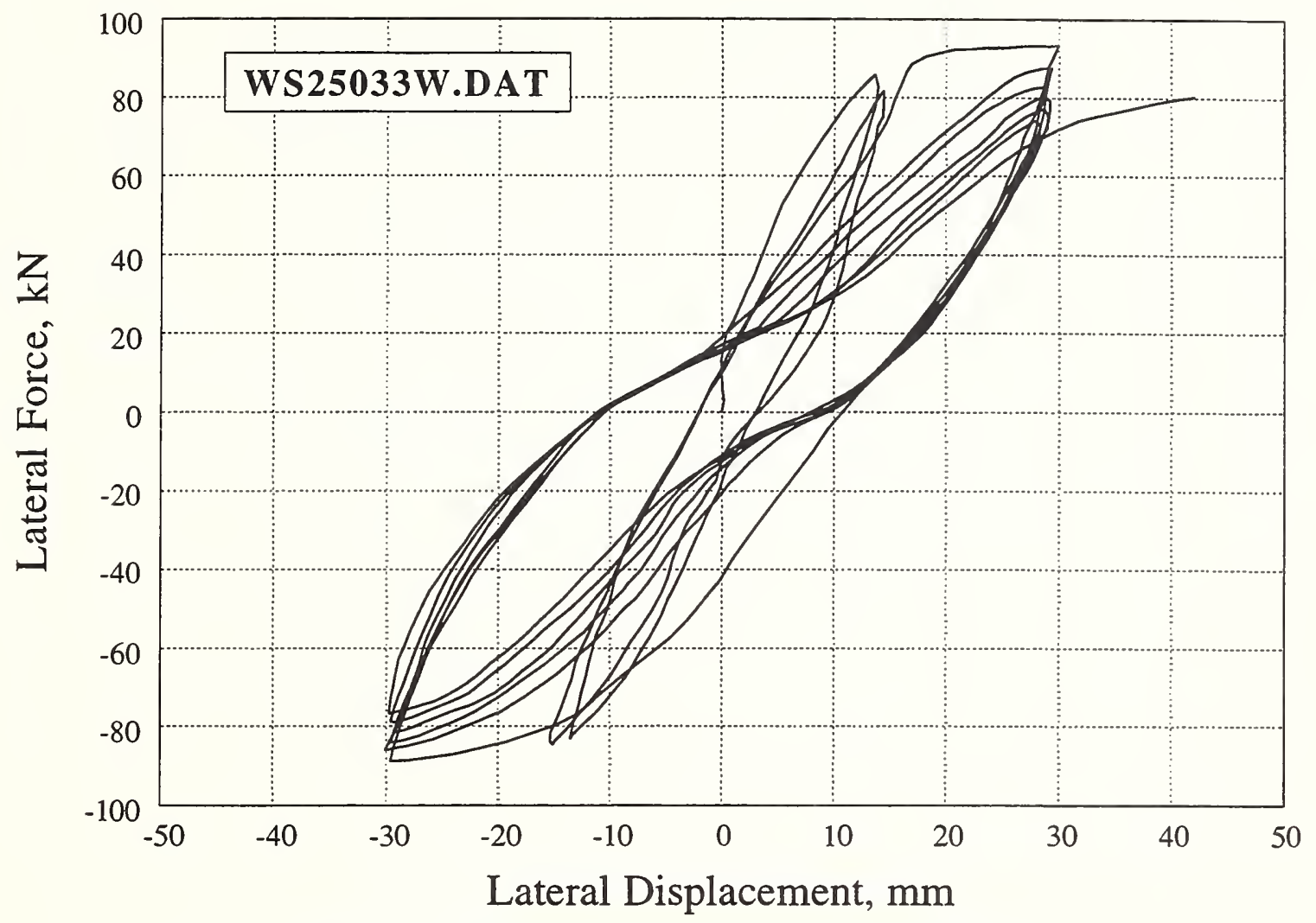

Figure 75. Specimen WS25033W of Wight 1973

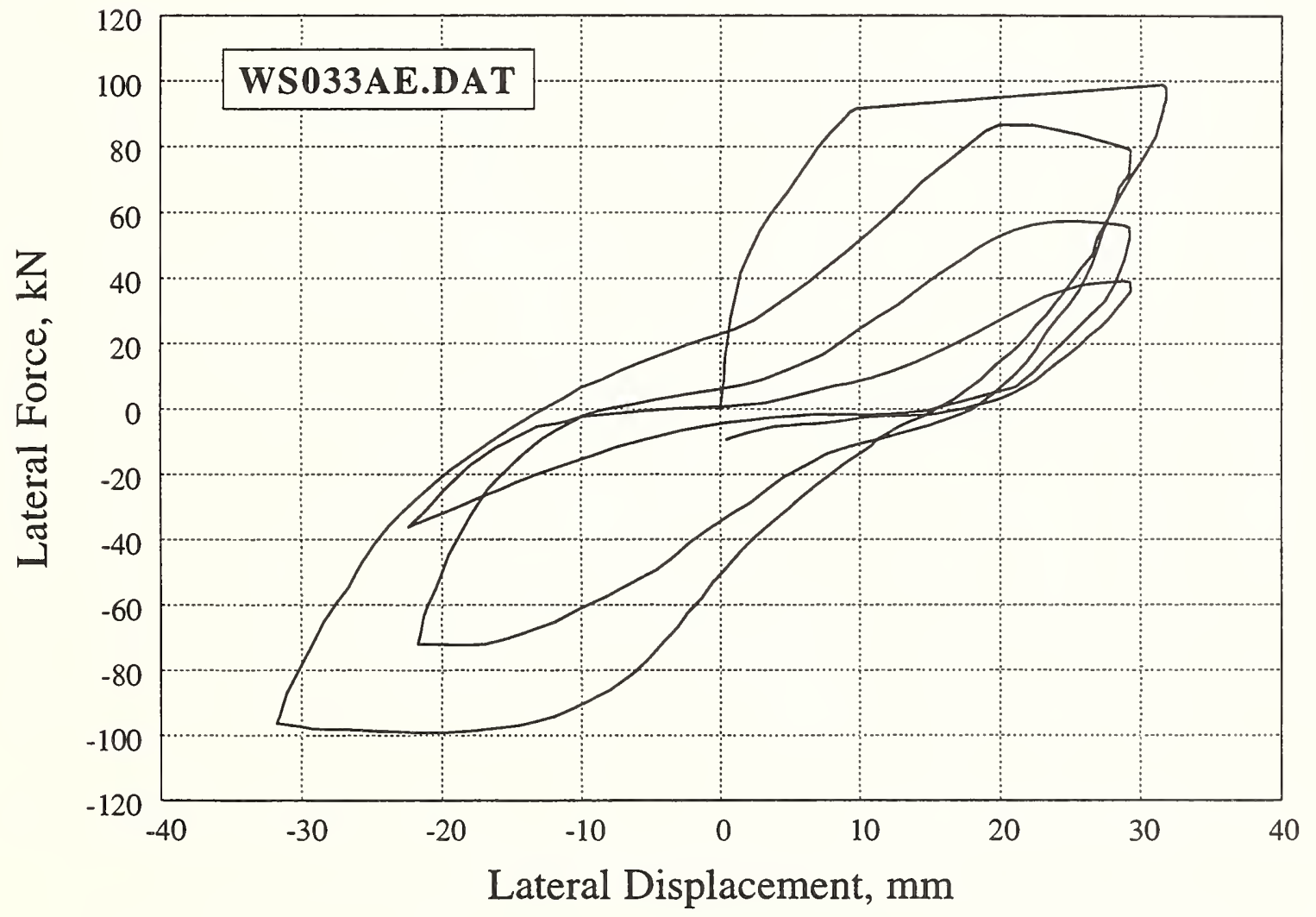

Figure 76. Specimen WS033AE of Wight 1973 


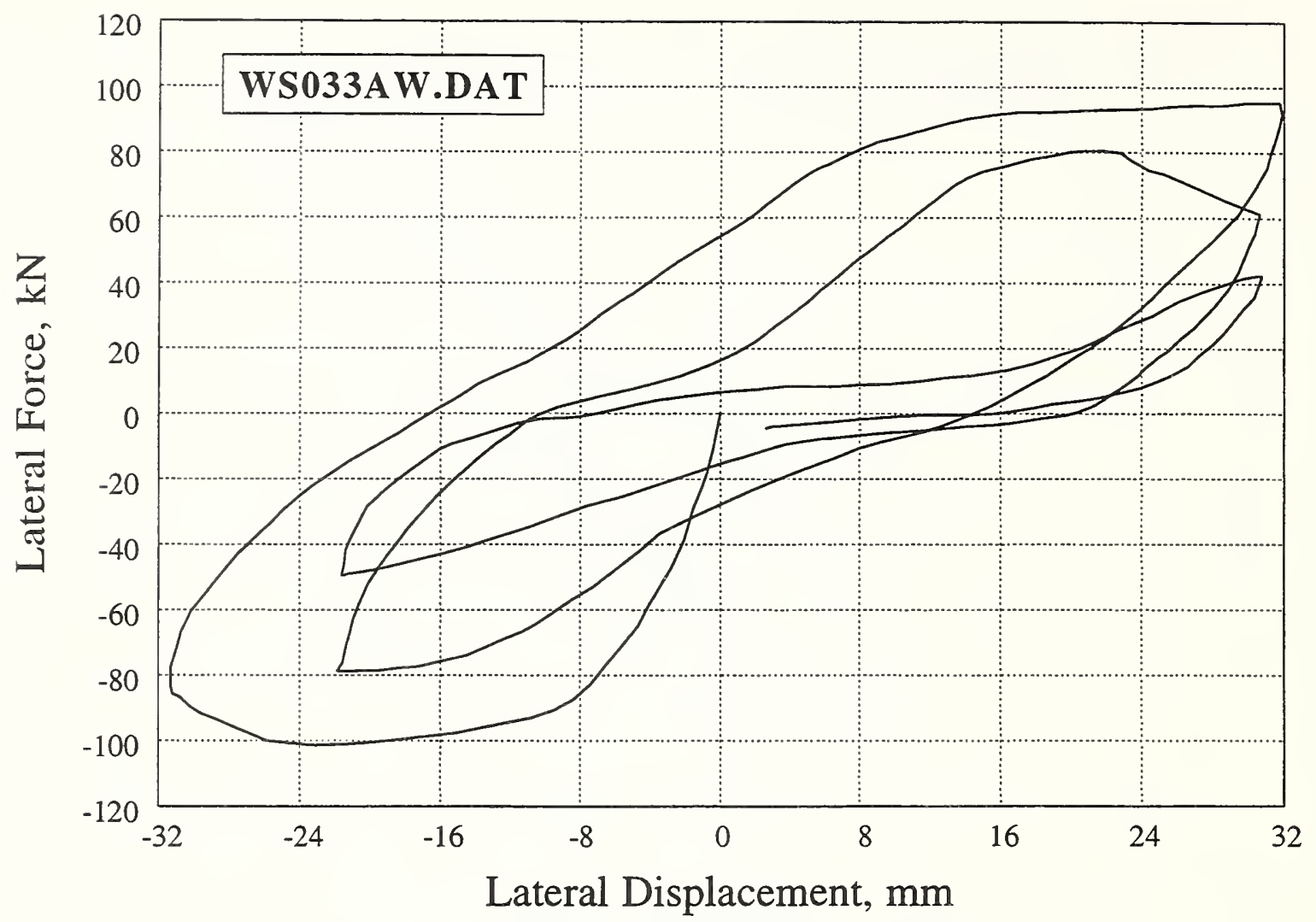

Figure 77. Specimen WS033AW of Wight 1973

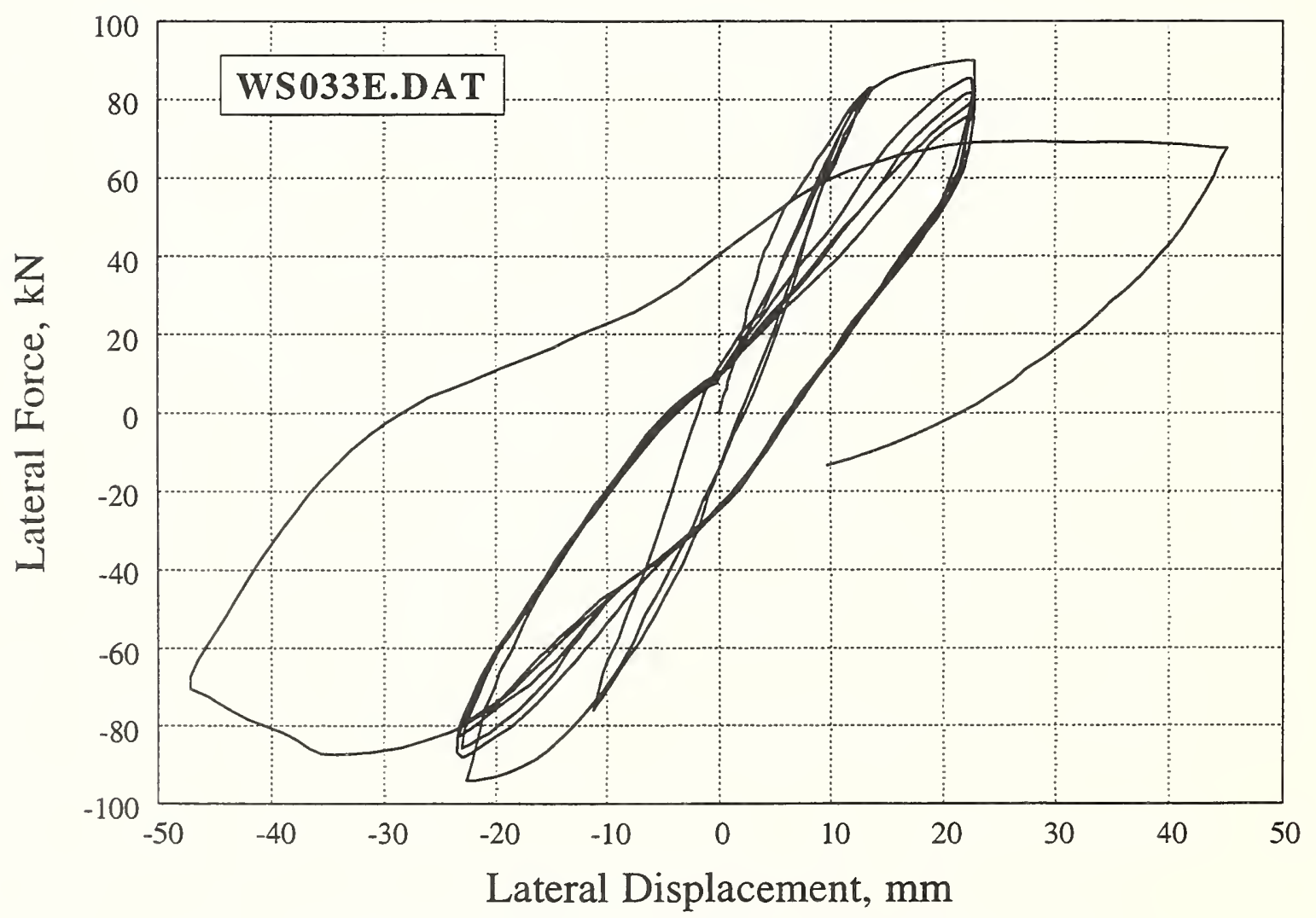

Figure 78. Specimen WS033E of Wight 1973 


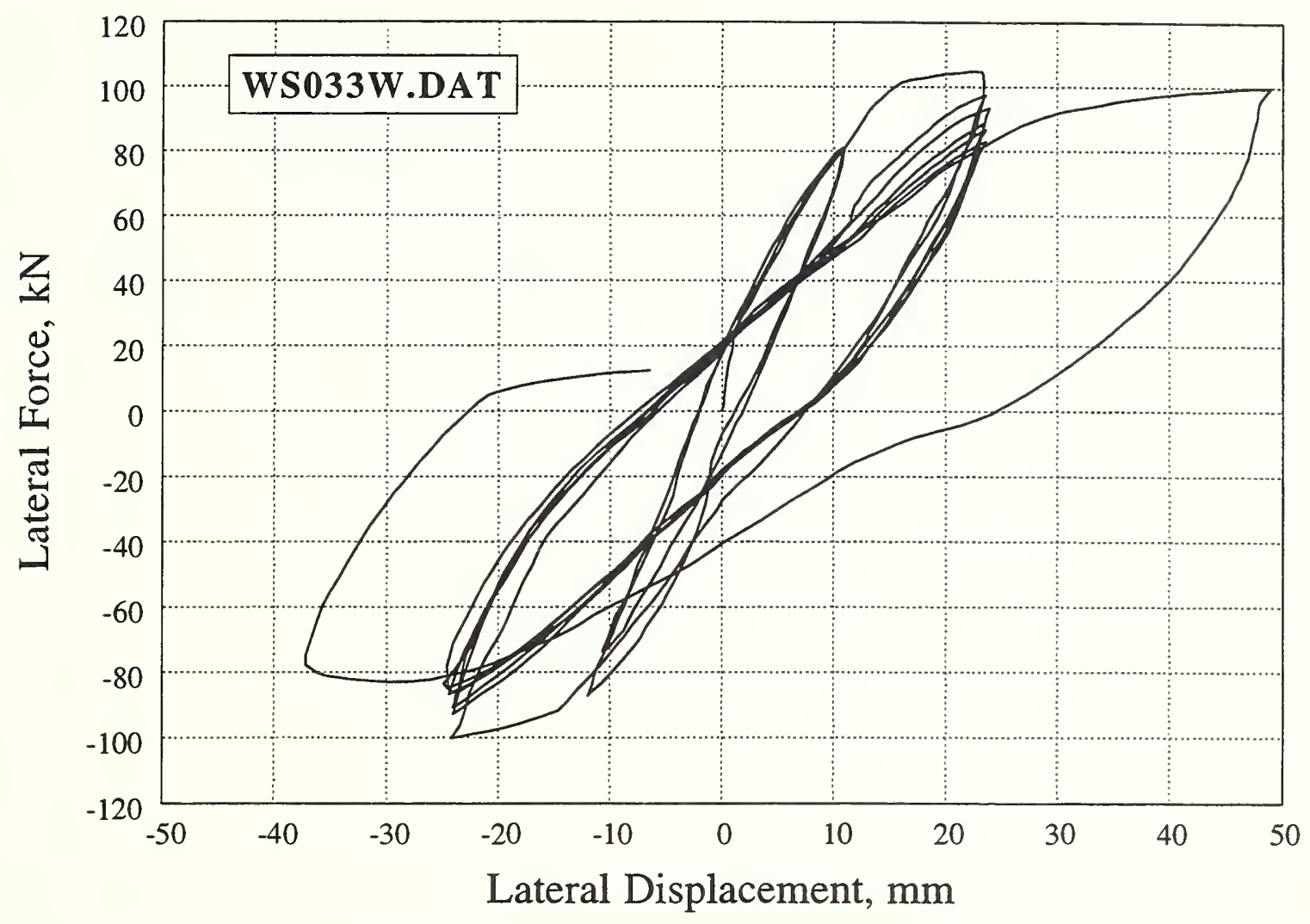

Figure 79. Specimen WS033W of Wight 1973

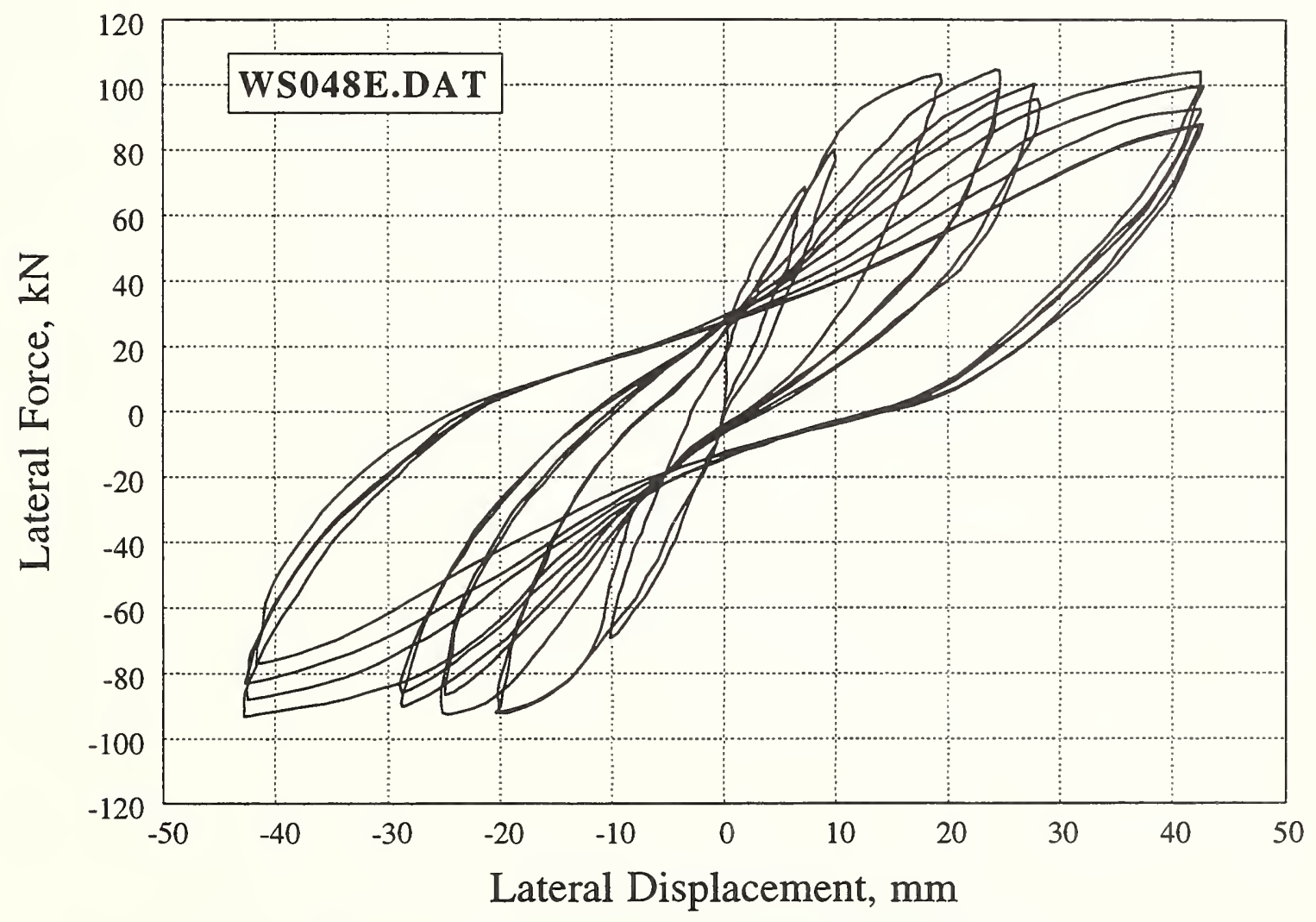

Figure 80. Specimen WS048E of Wight 1973 


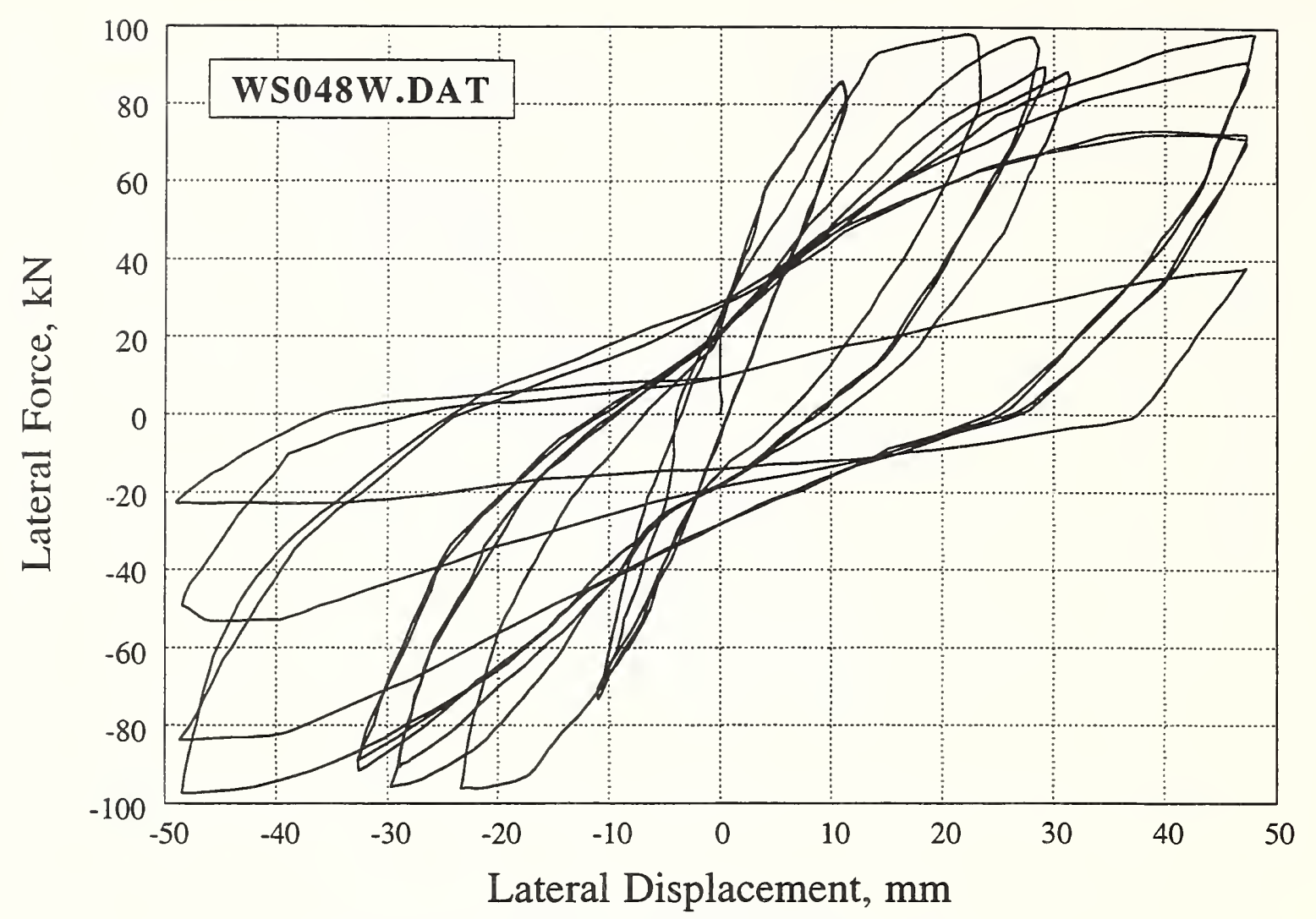

Figure 81. Specimen WS048W of Wight 1973

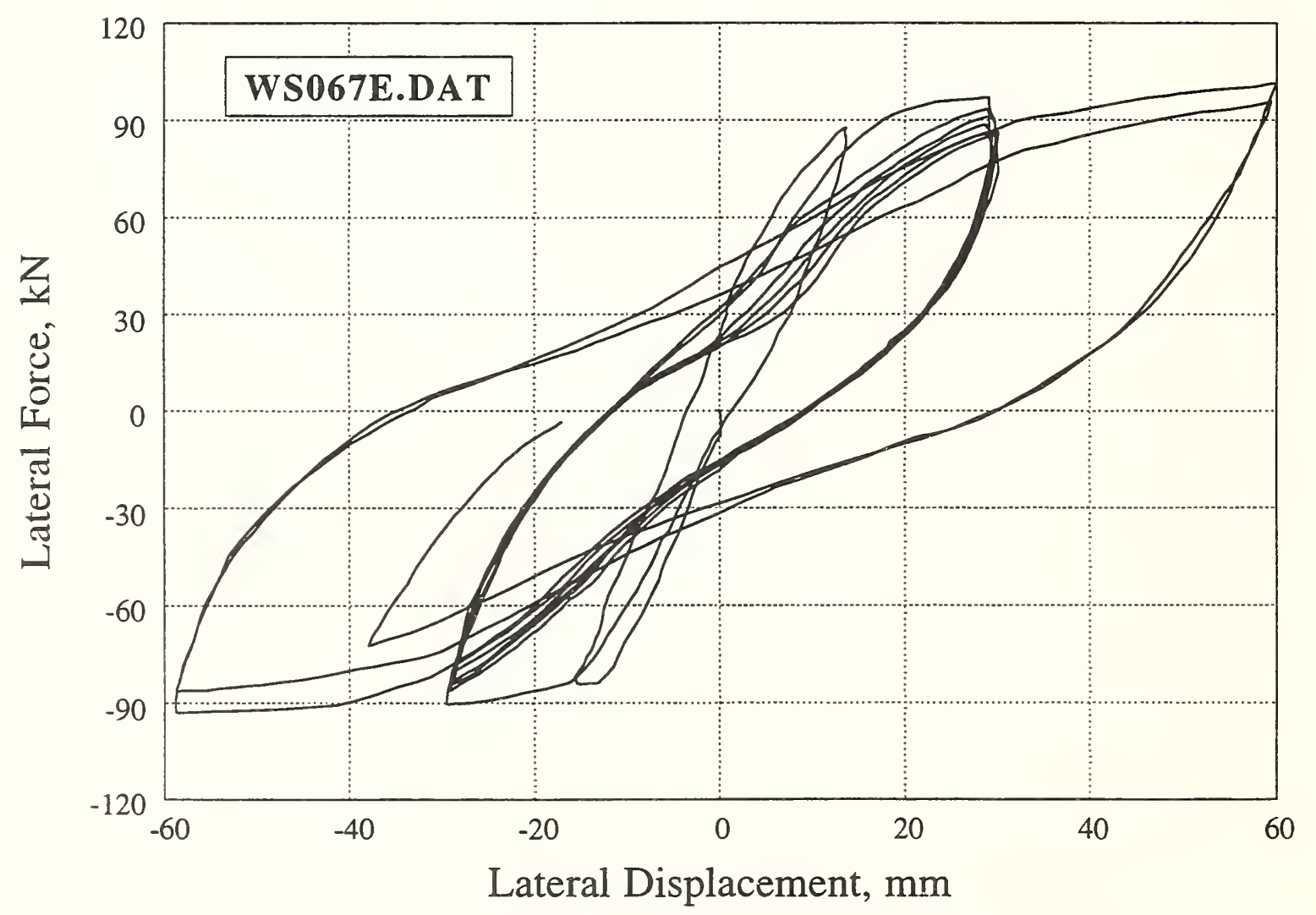

Figure 82. Specimen WS067E of Wight 1973 


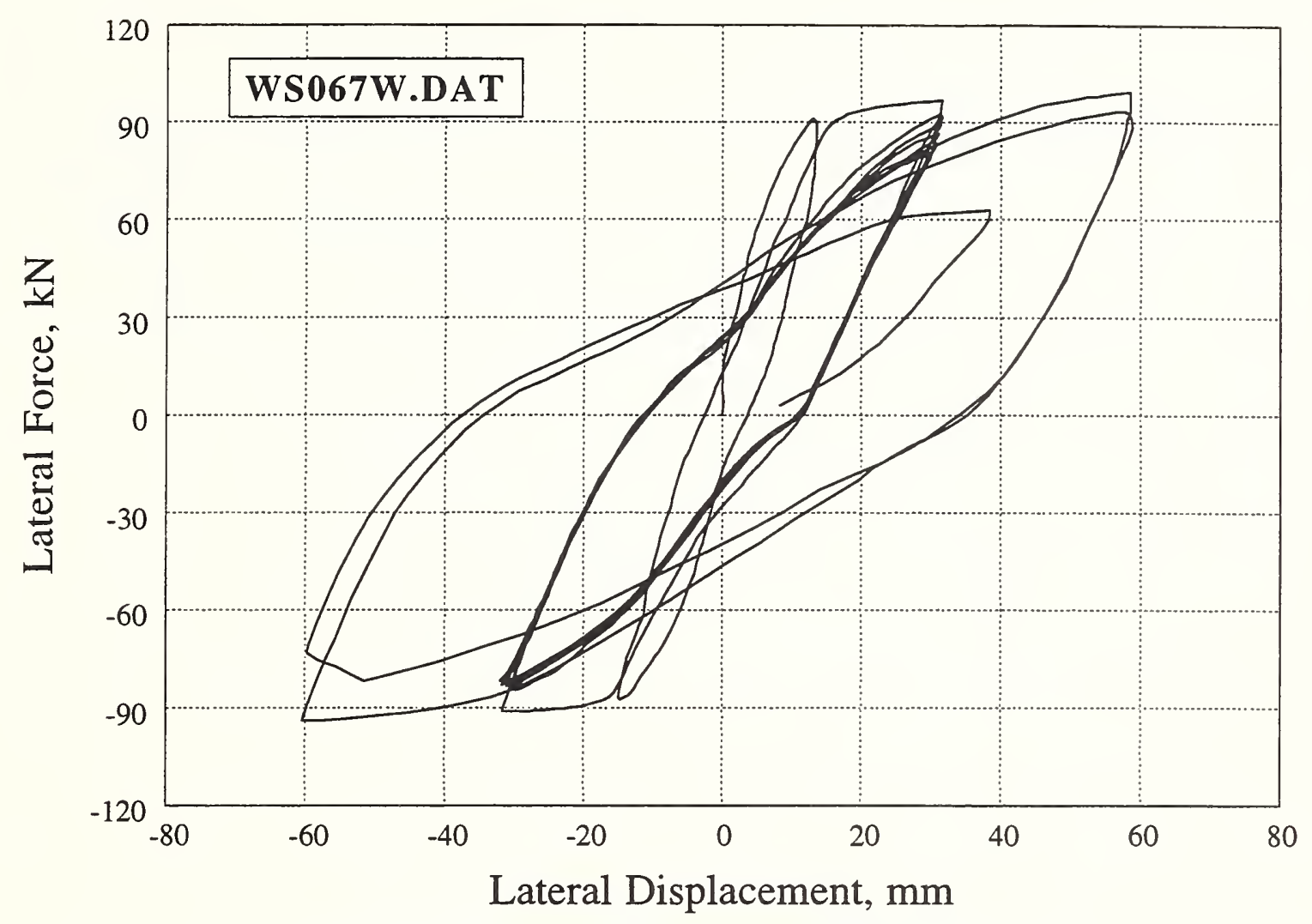

Figure 83. Specimen WS067W of Wight 1973

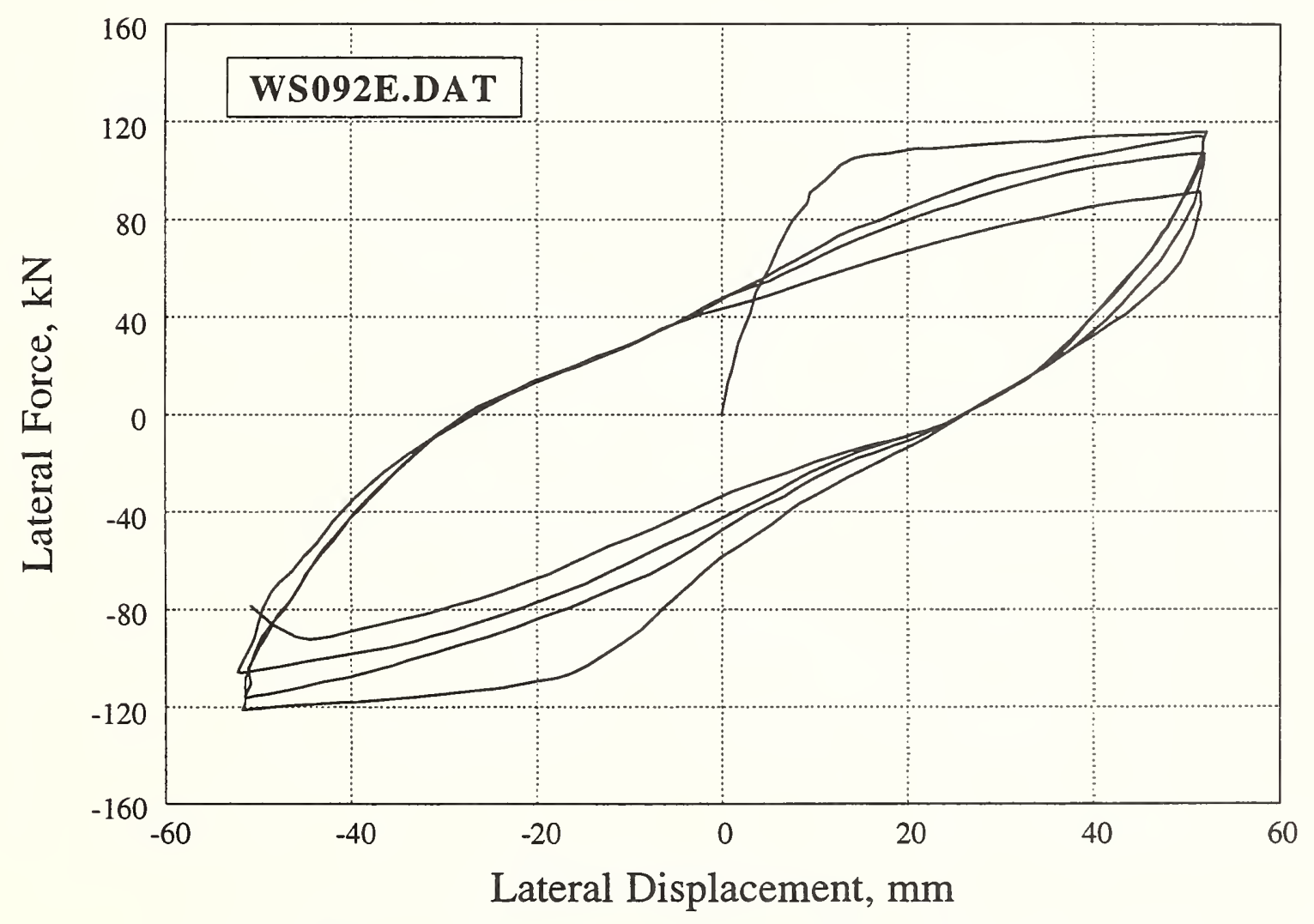

Figure 84. Specimen WS092E of Wight 1973 


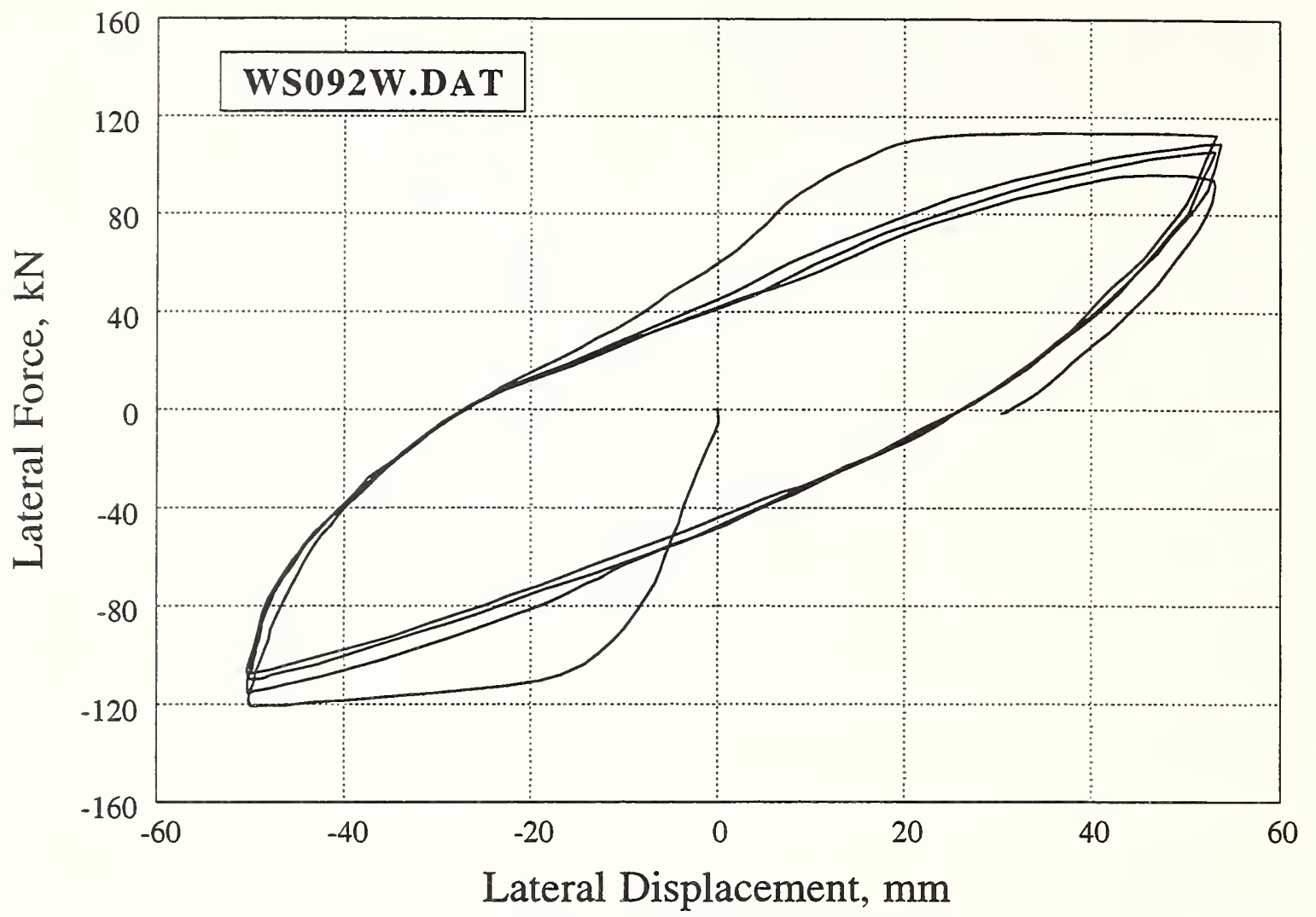

Figure 85. Specimen WS092W of Wight 1973

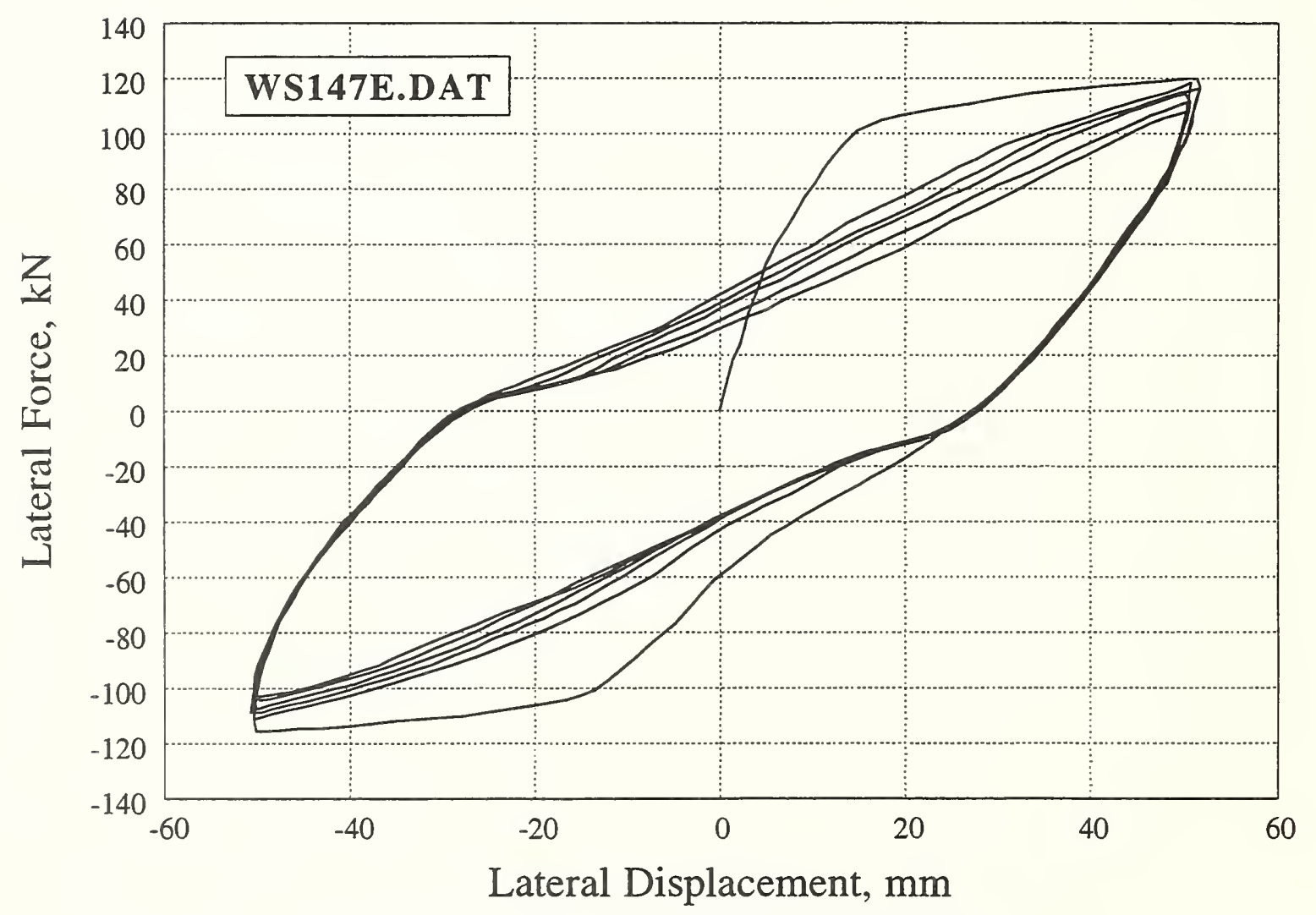

Figure 86. Specimen WS147E of Wight 1973 


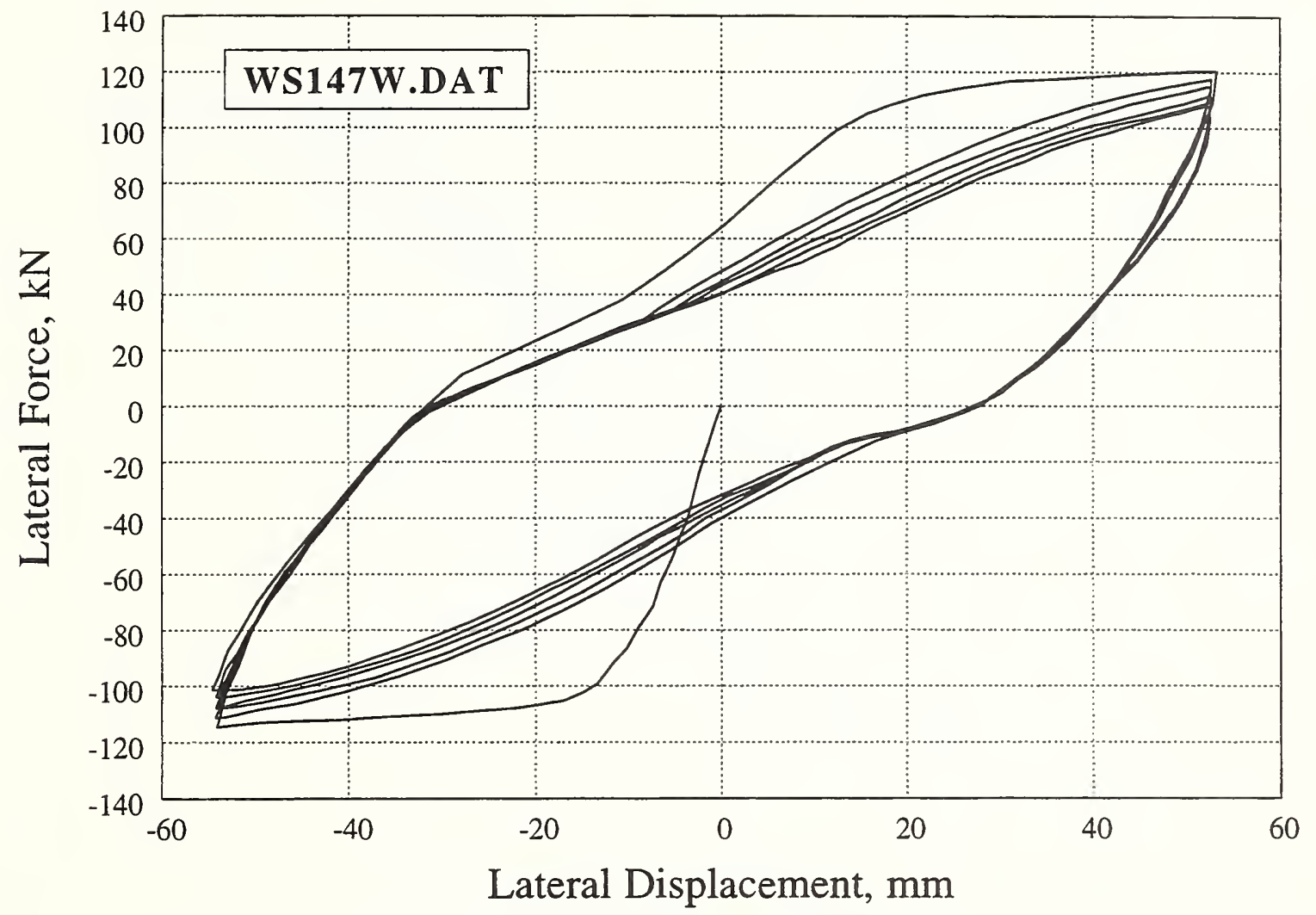

Figure 87. Specimen WS147W of Wight 1973

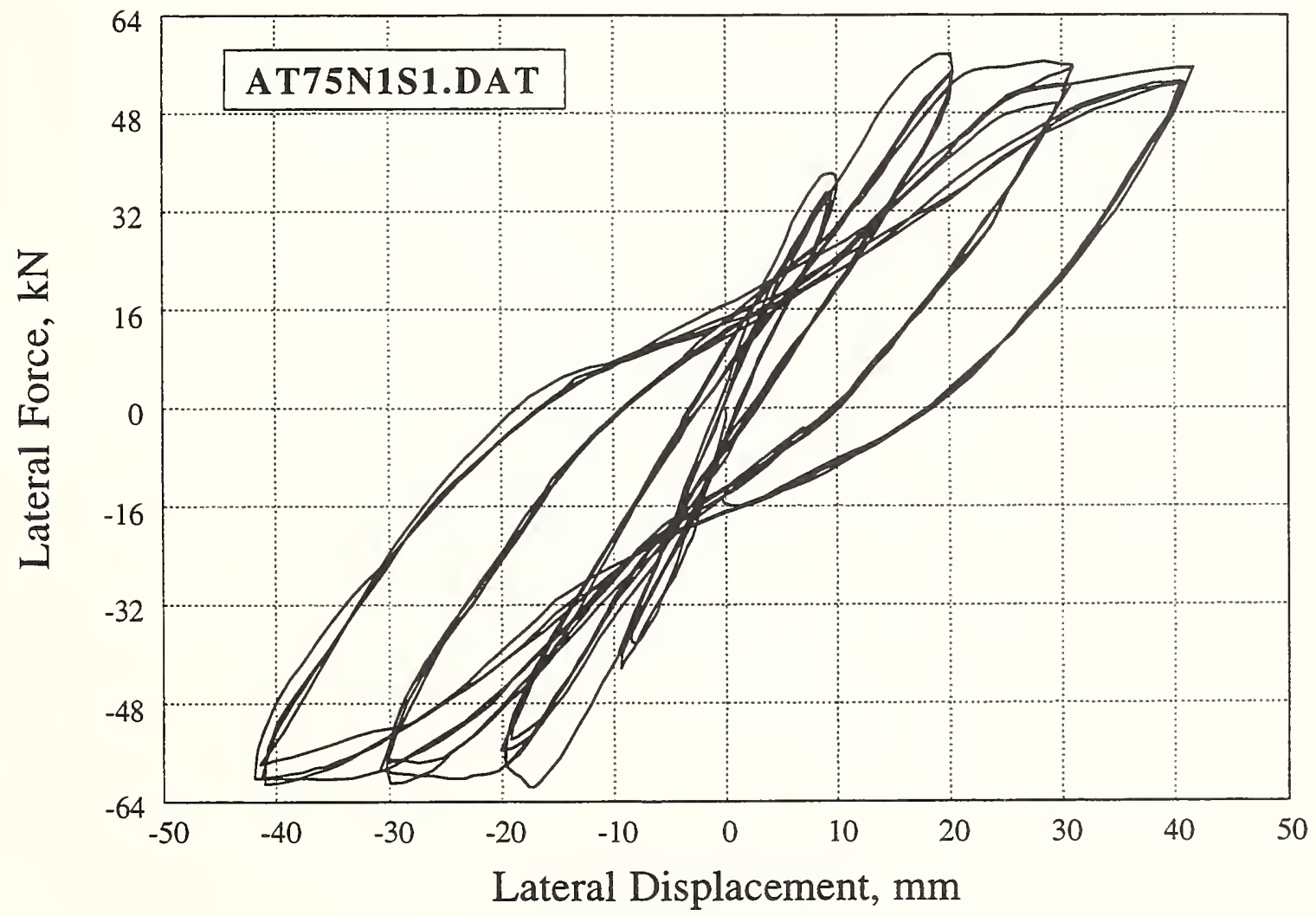

Figure 88. Specimen 1 of Atalay 1975 


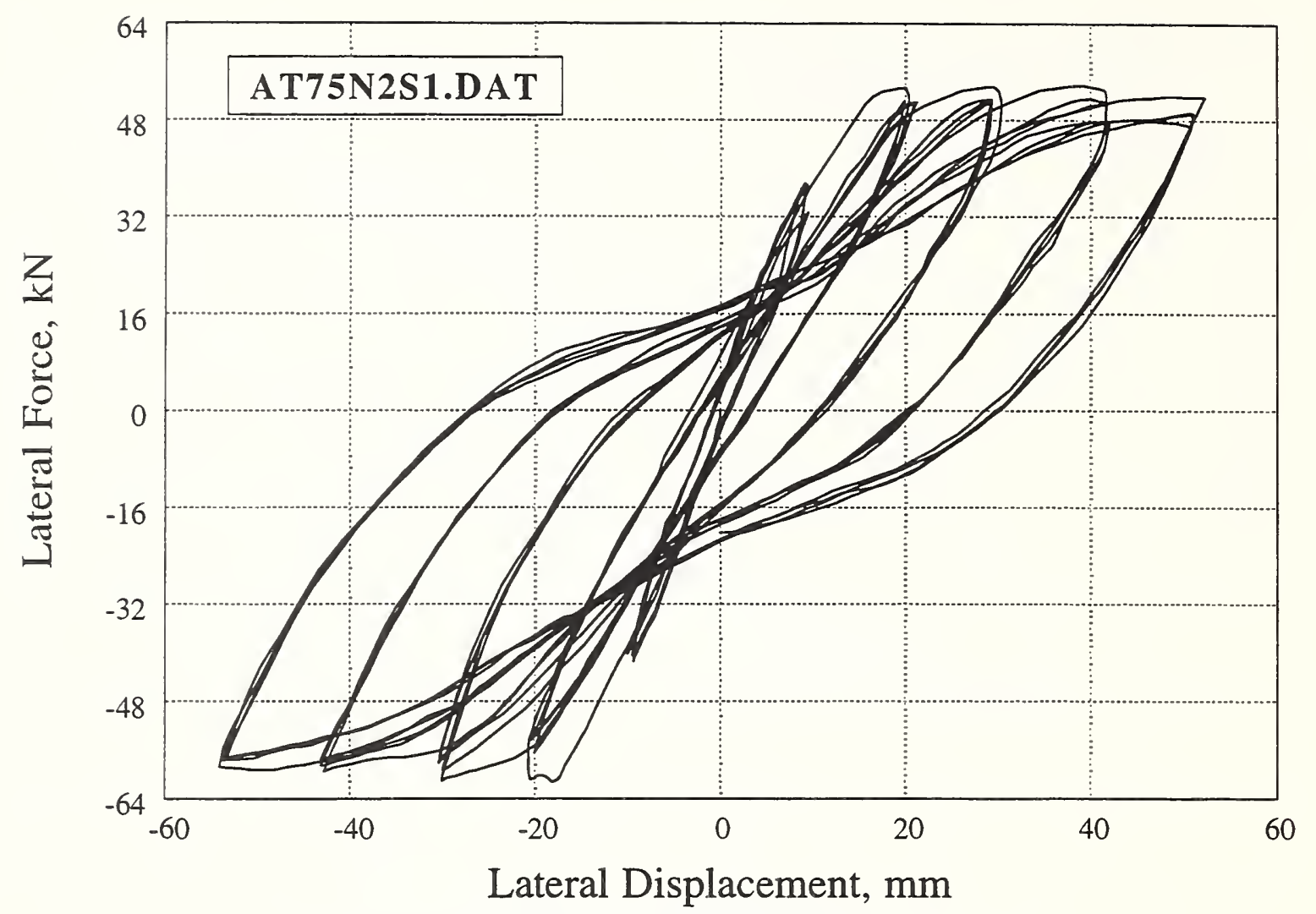

Figure 89. Specimen 2 of Atalay 1975

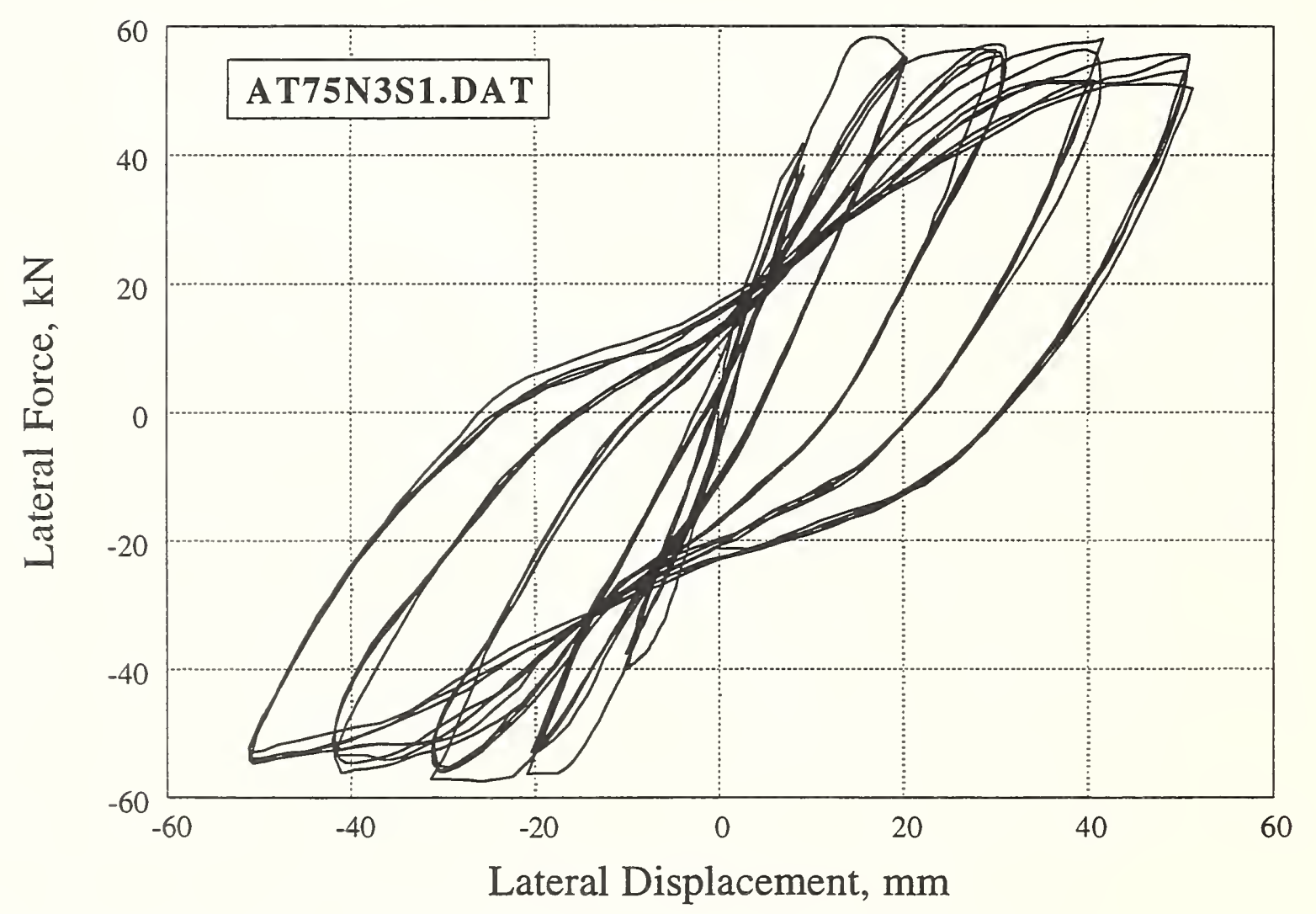

Figure 90. Specimen 3 of Atalay 1975 


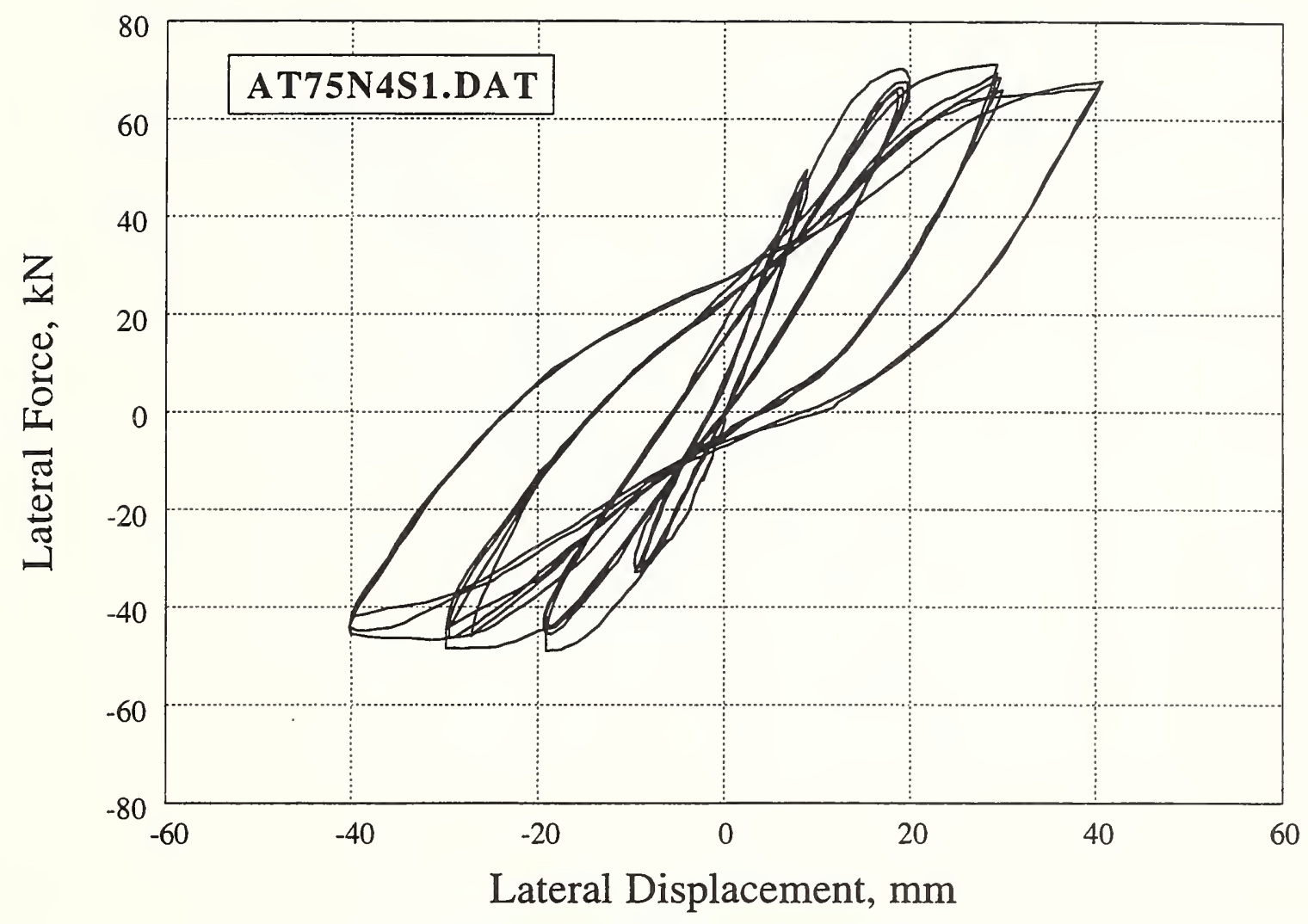

Figure 91. Specimen 4 of Atalay 1975

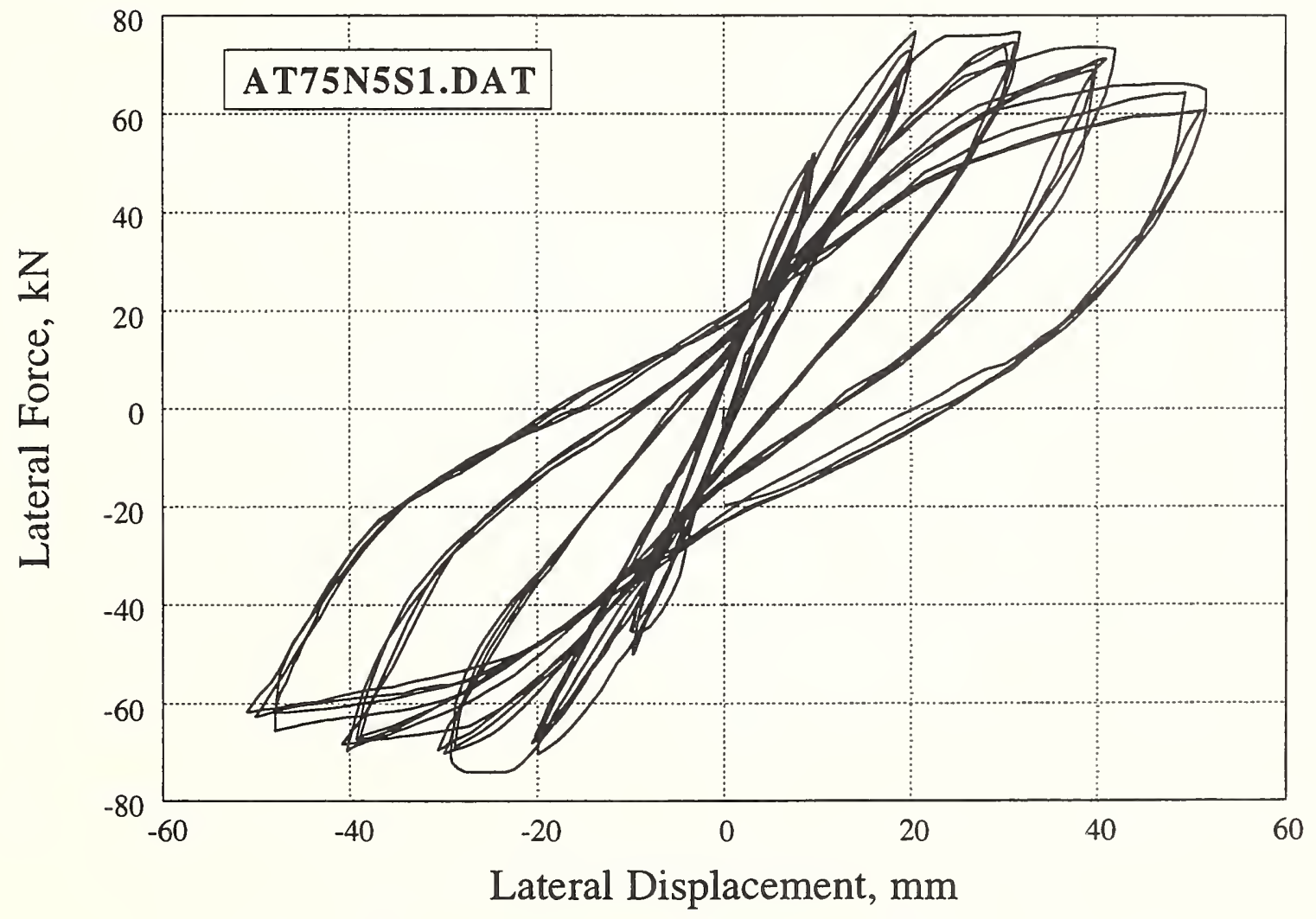

Figure 92. Specimen 5 of Atalay 1975 


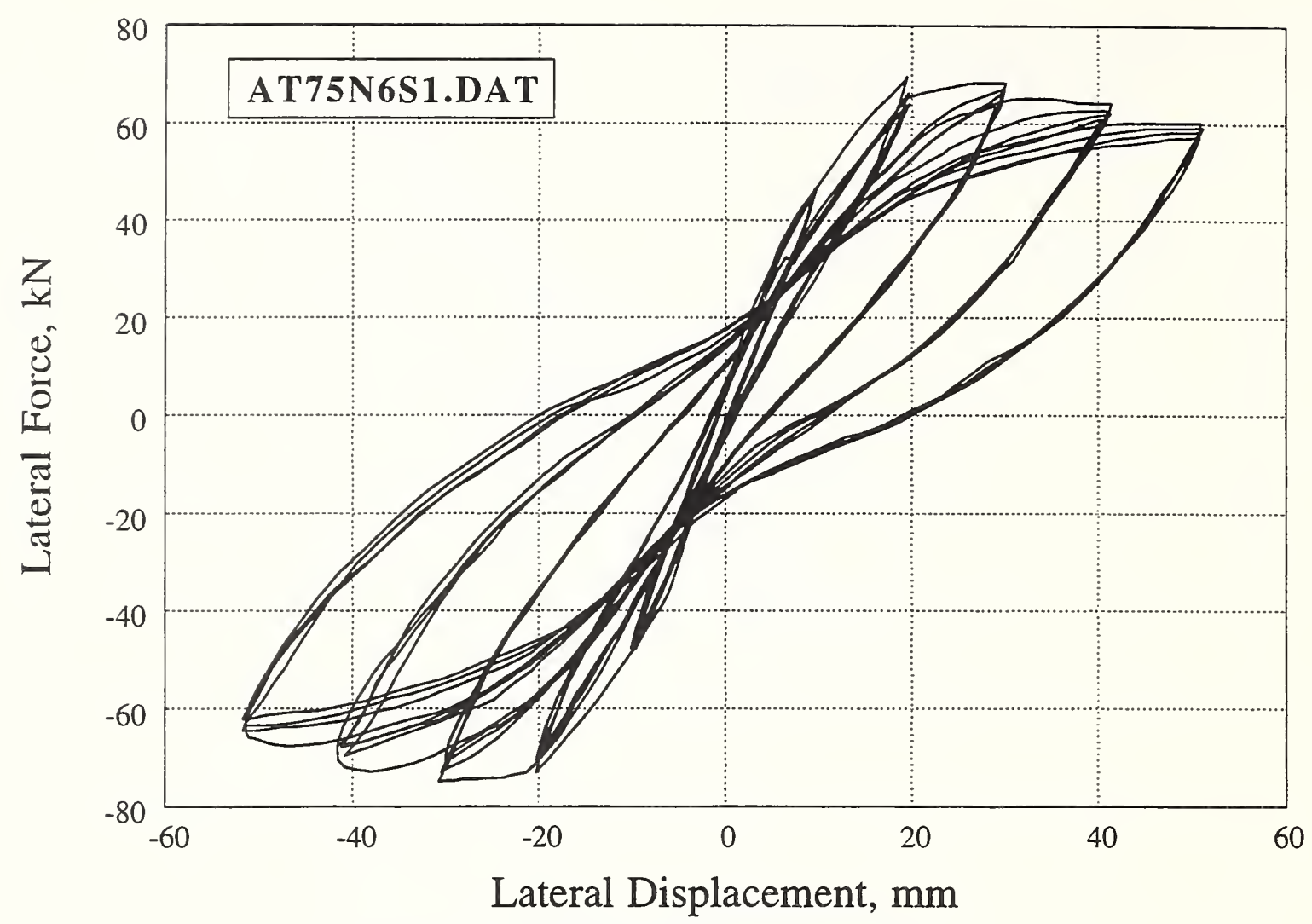

Figure 93. Specimen 6 of Atalay 1975

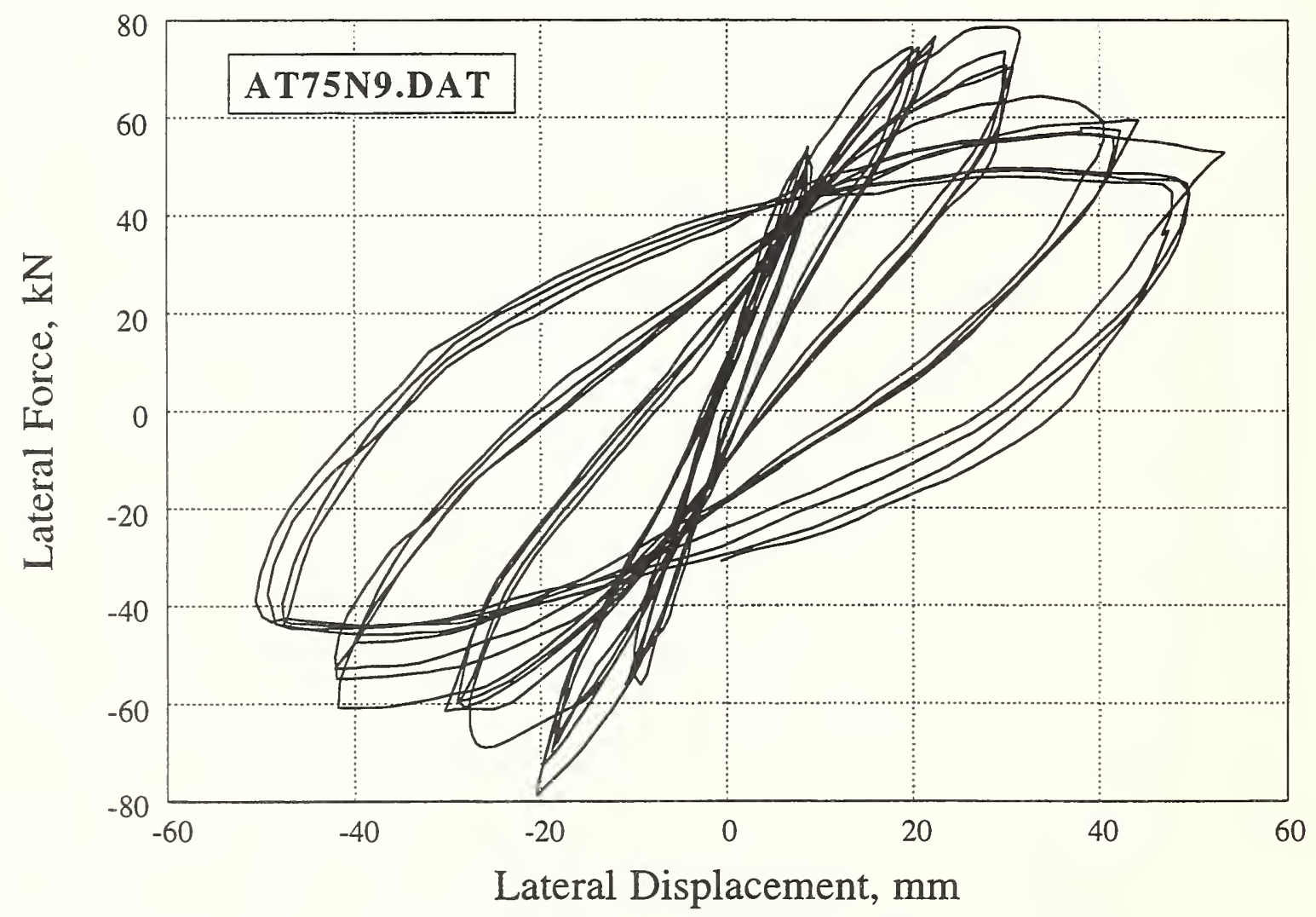

Figure 94. Specimen 9 of Atalay 1975 


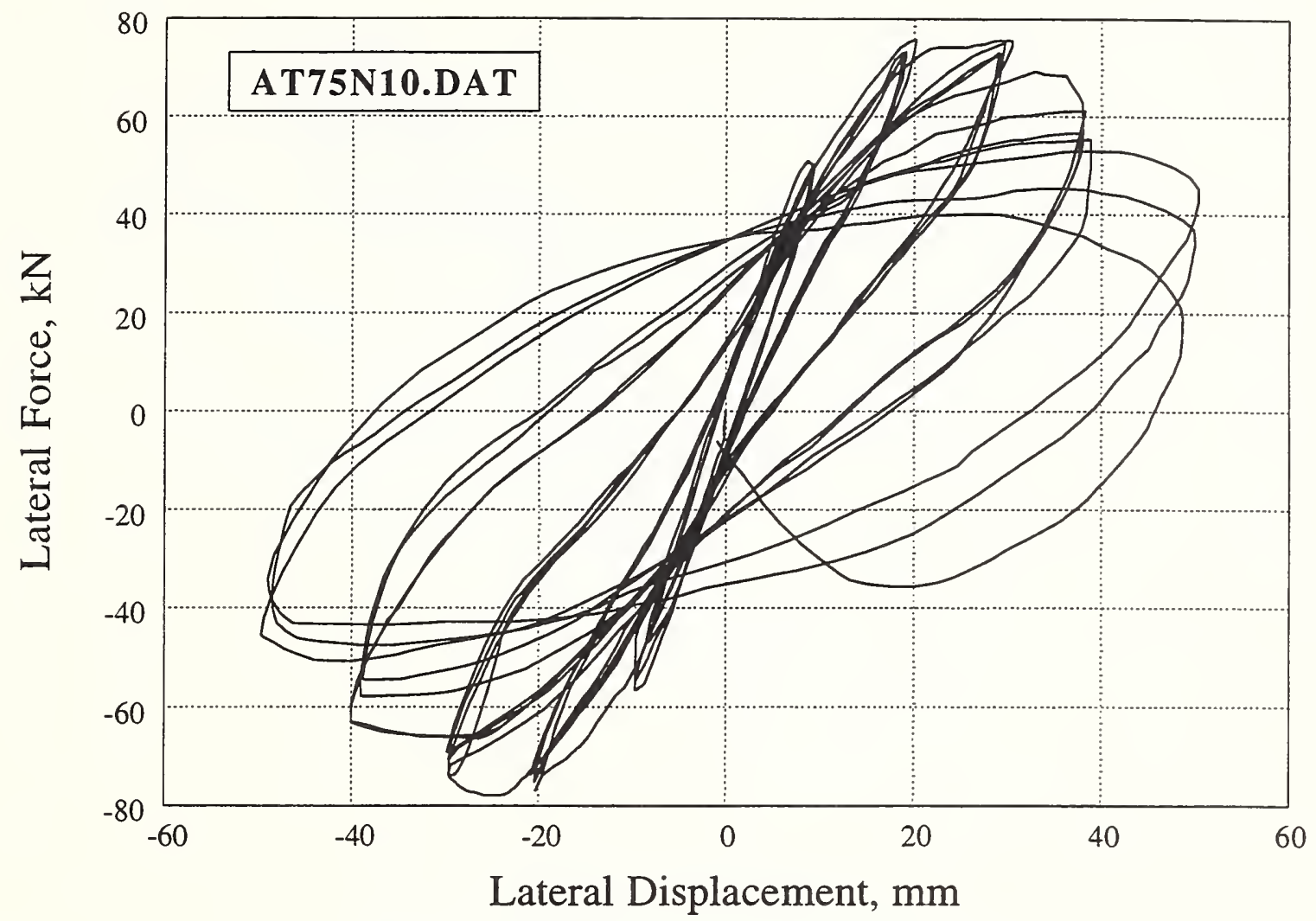

Figure 95. Specimen 10 of Atalay 1975

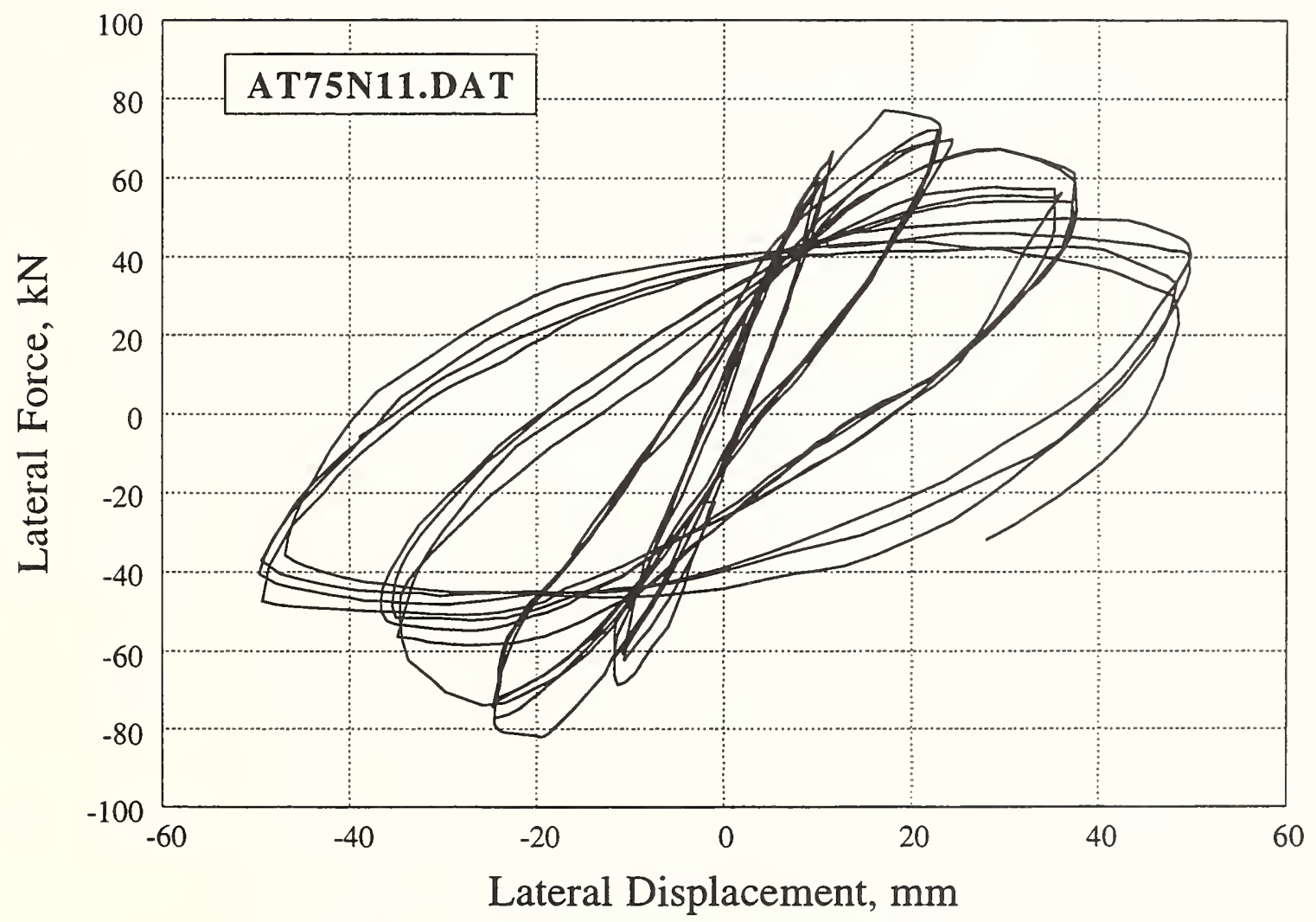

Figure 96. Specimen 11 of Atalay 1975 


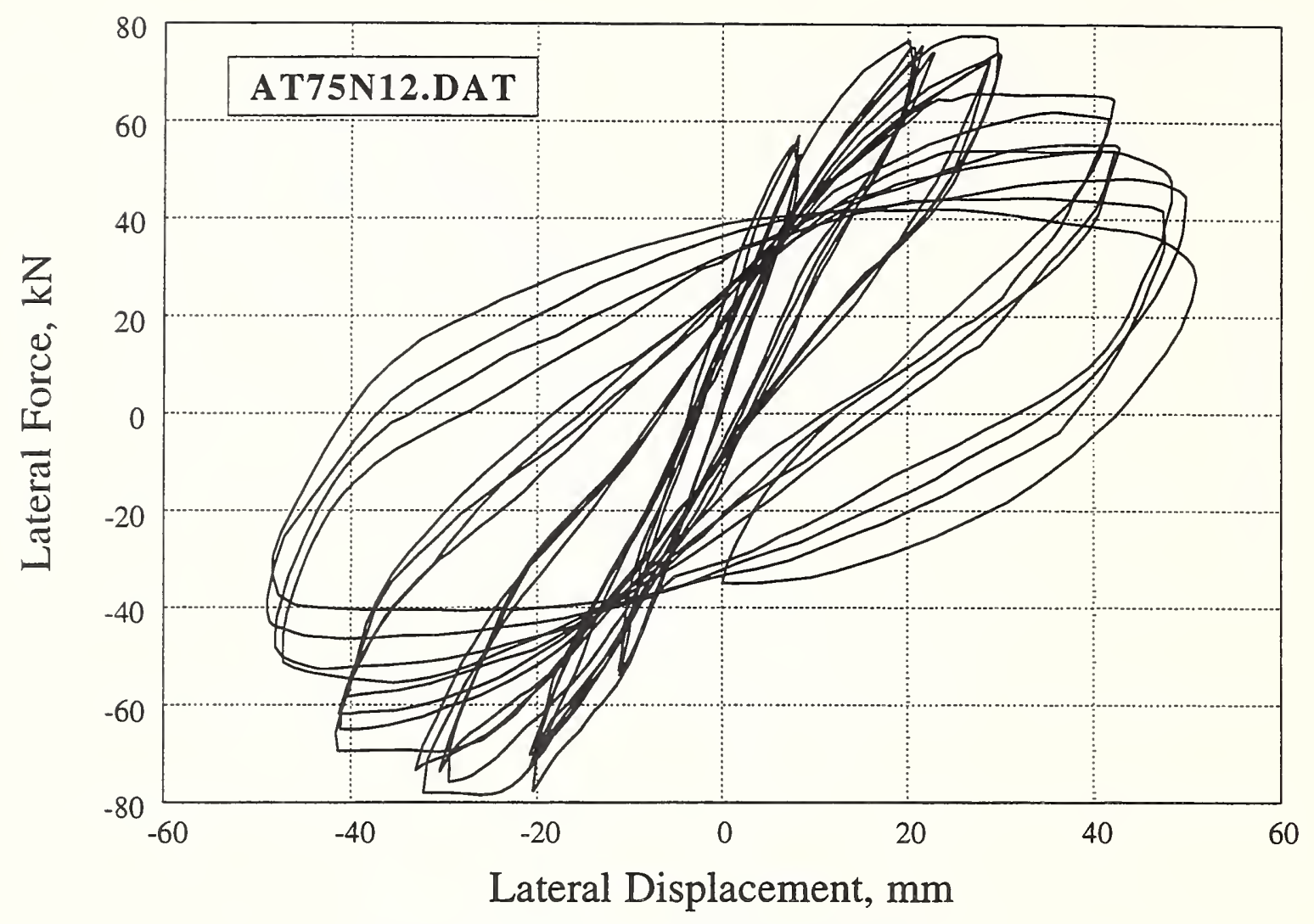

Figure 97. Specimen 12 of Atalay 1975

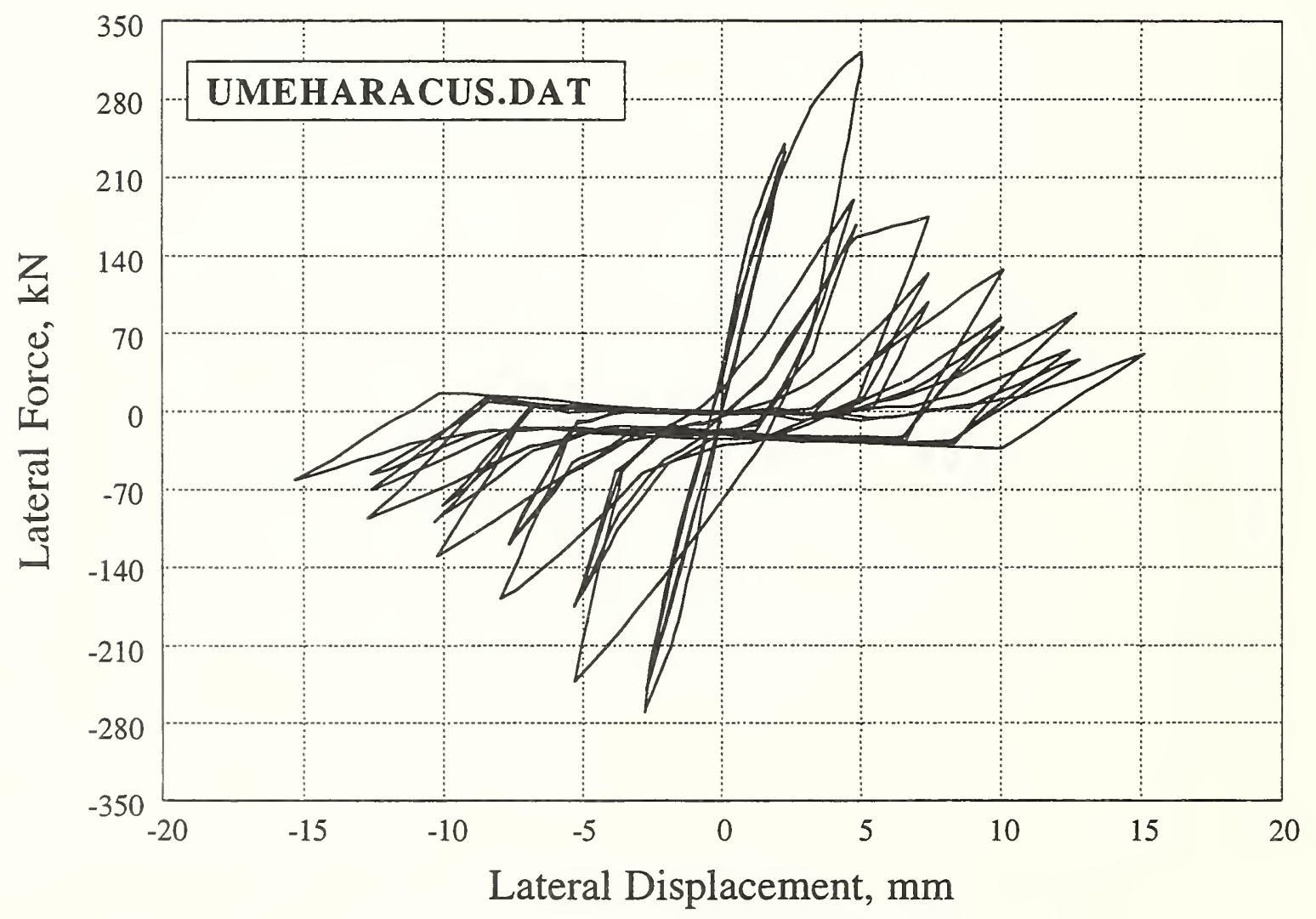

Figure 98. Specimen CUS of Umehara 1982 


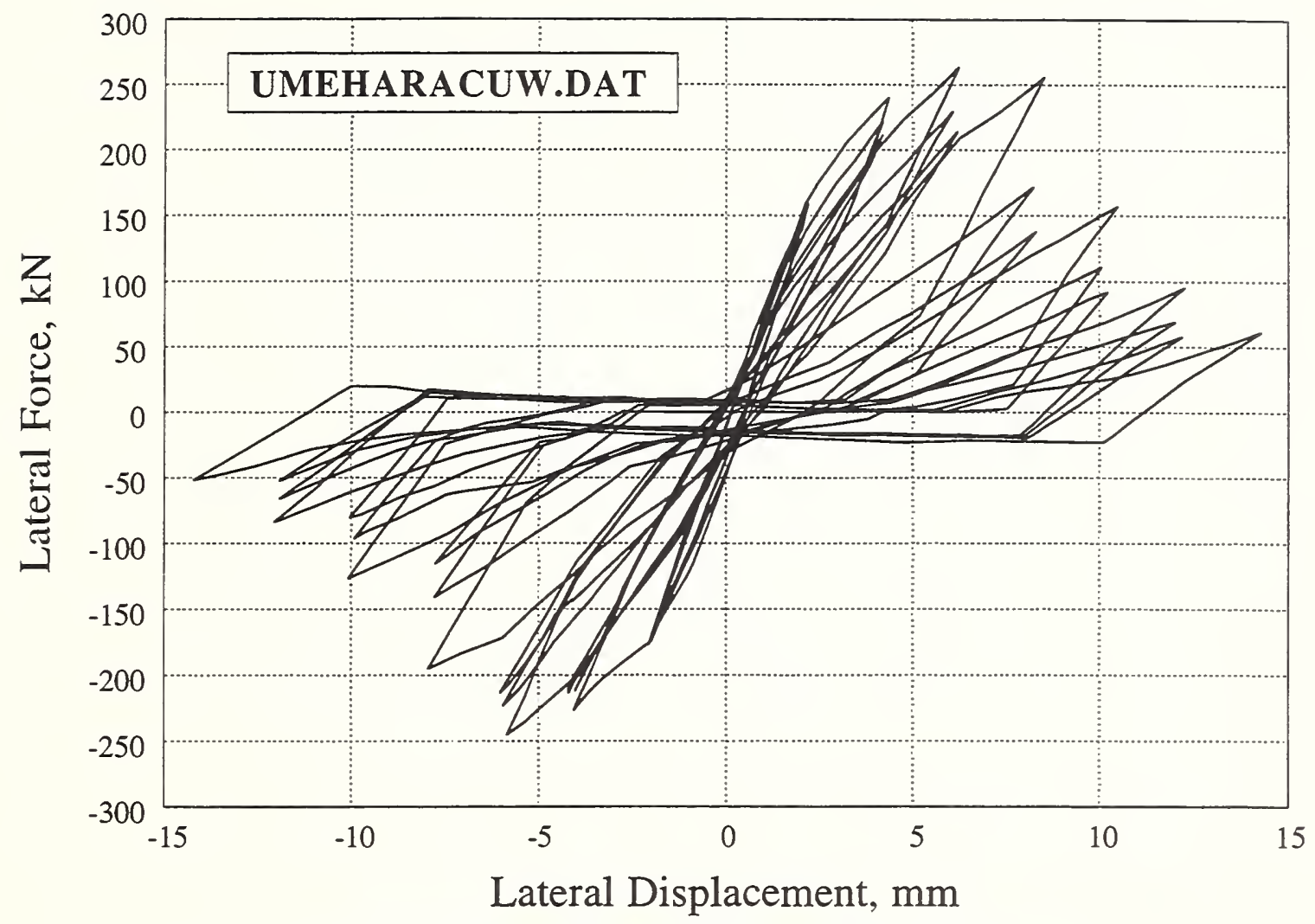

Figure 99. Specimen CUW of Umehara 1982

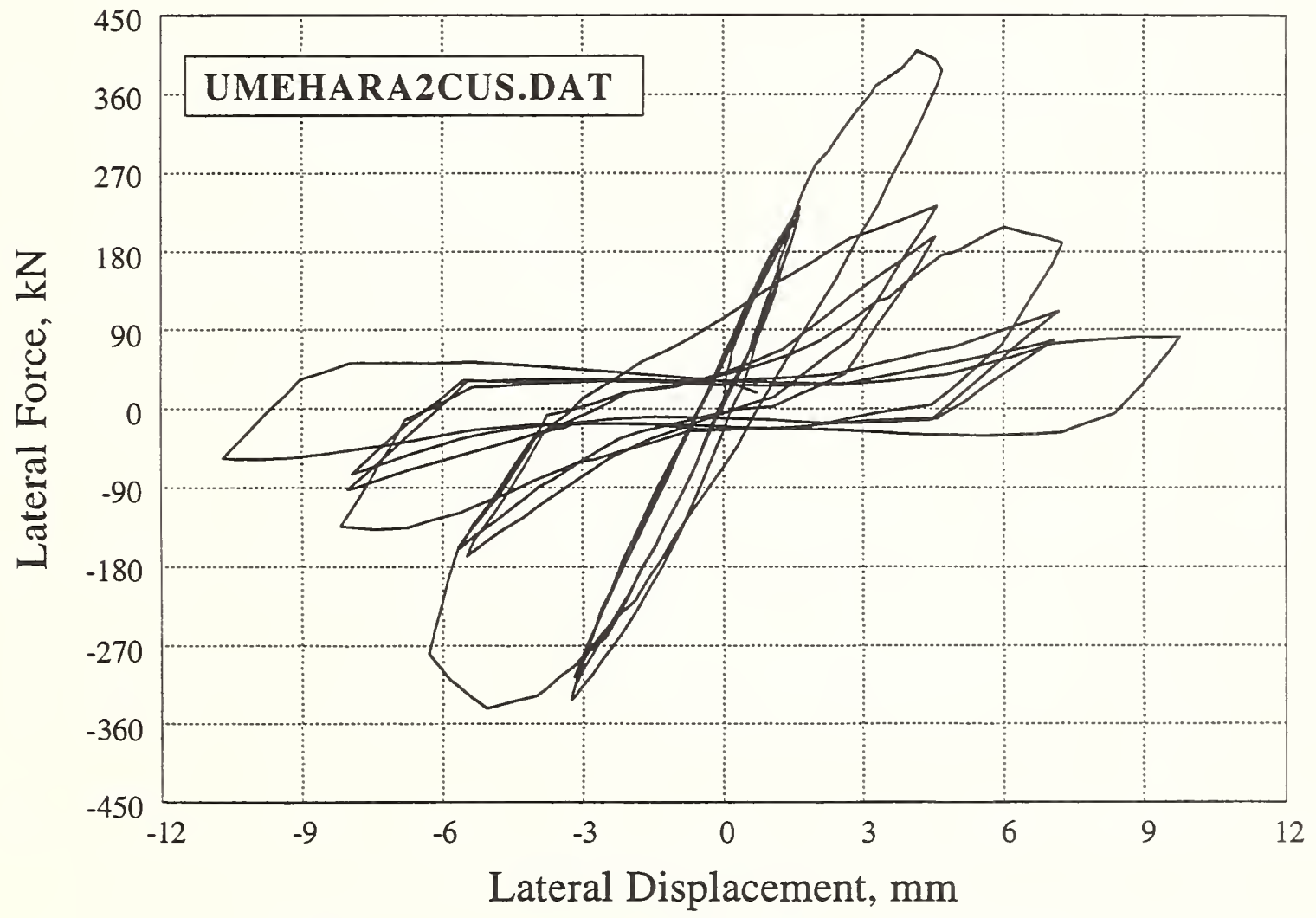

Figure 100. Specimen 2CUS of Umehara 1982 


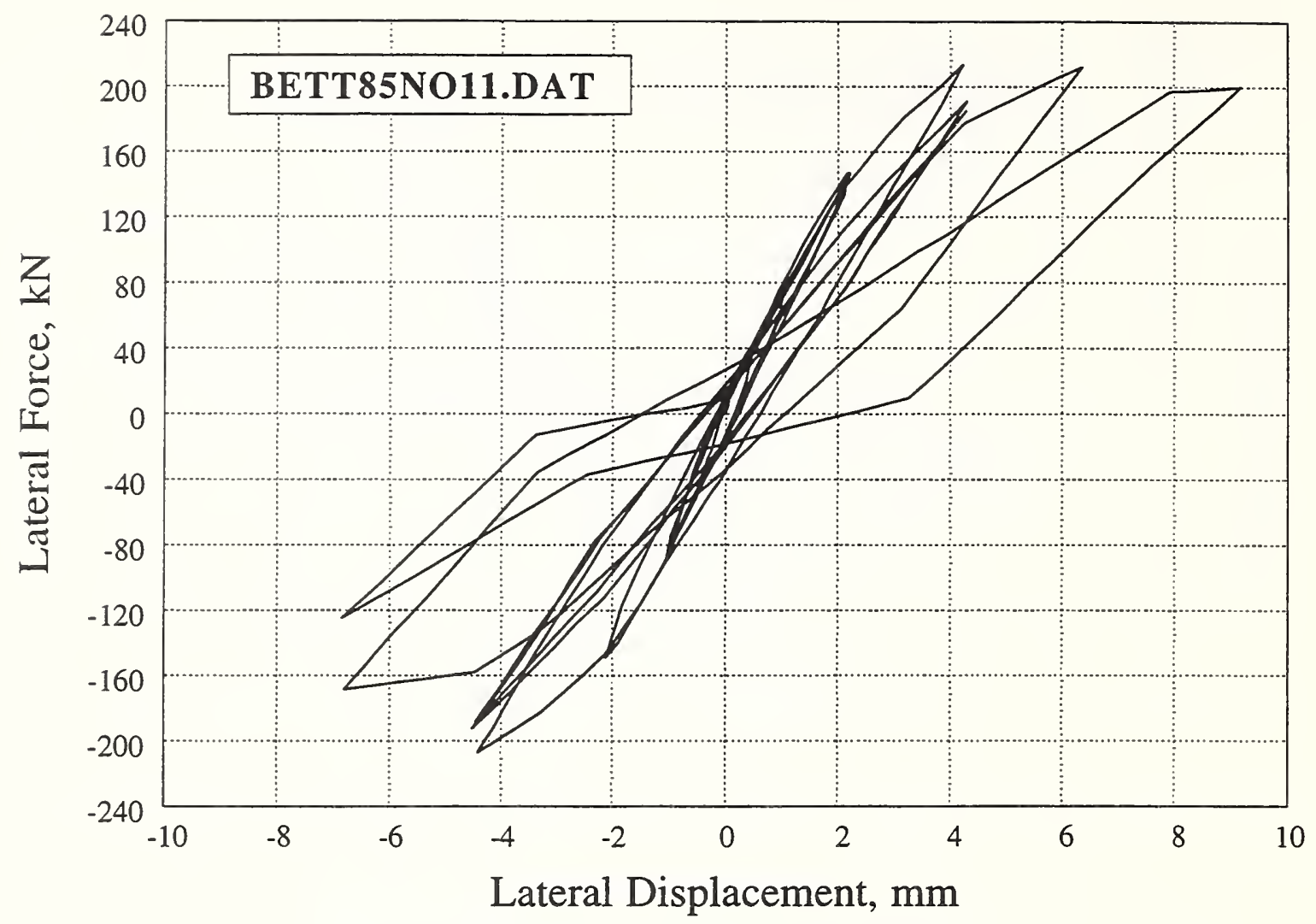

Figure 101. Specimen 11 of Bett 1985

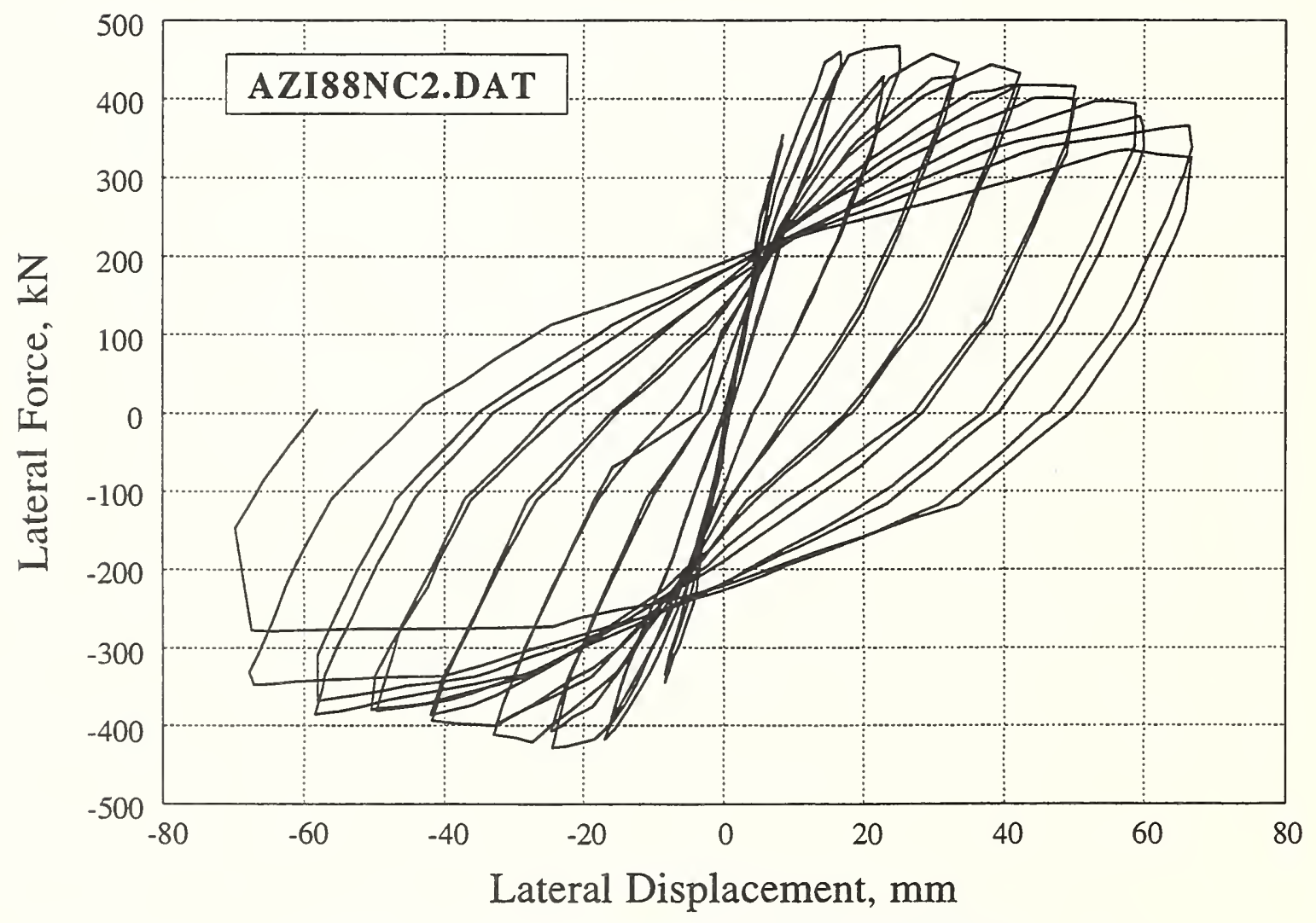

Figure 102. Specimen NC-2 of Azizinamini 1988 


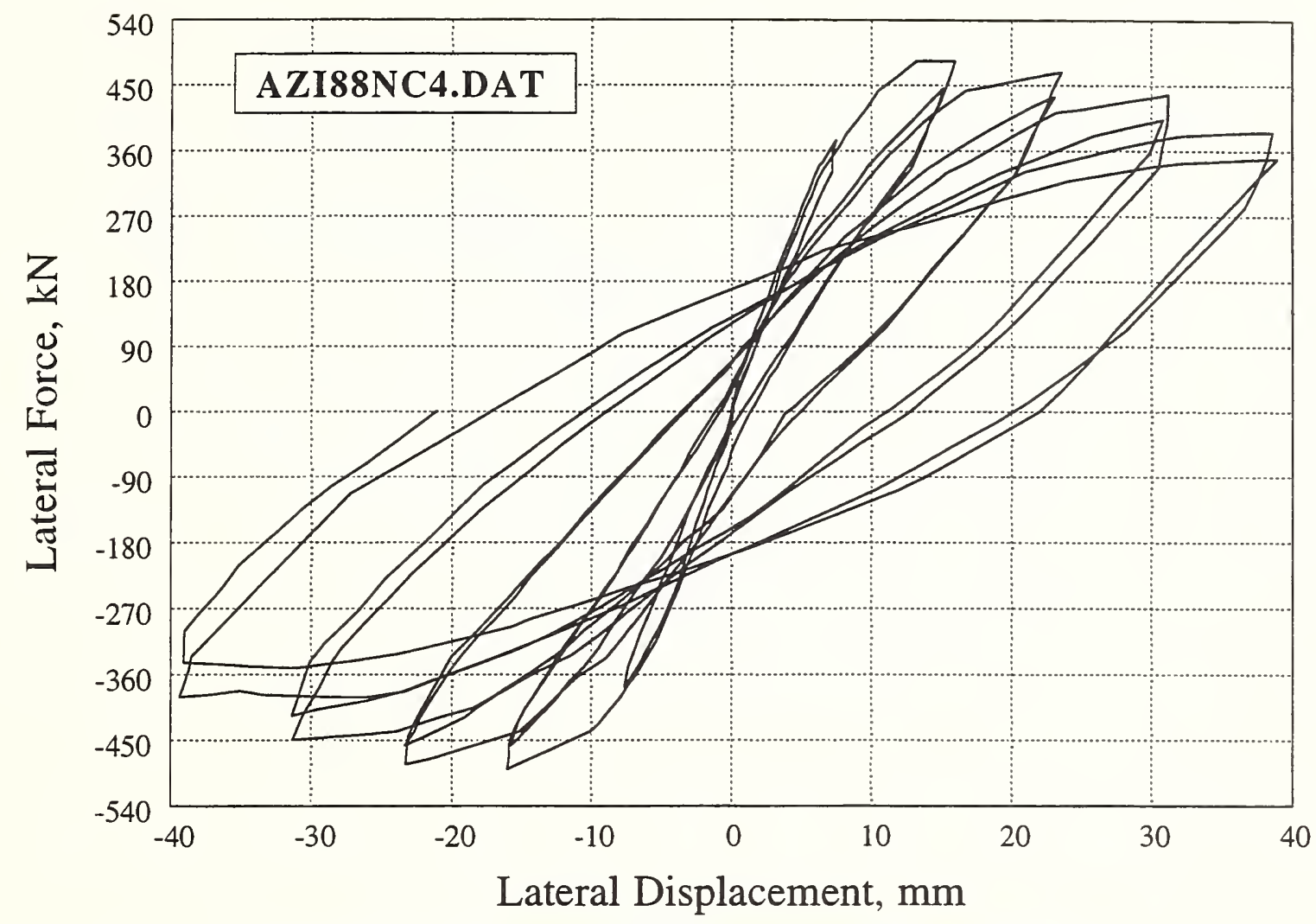

Figure 103. Specimen NC-4 of Azizinamini 1988

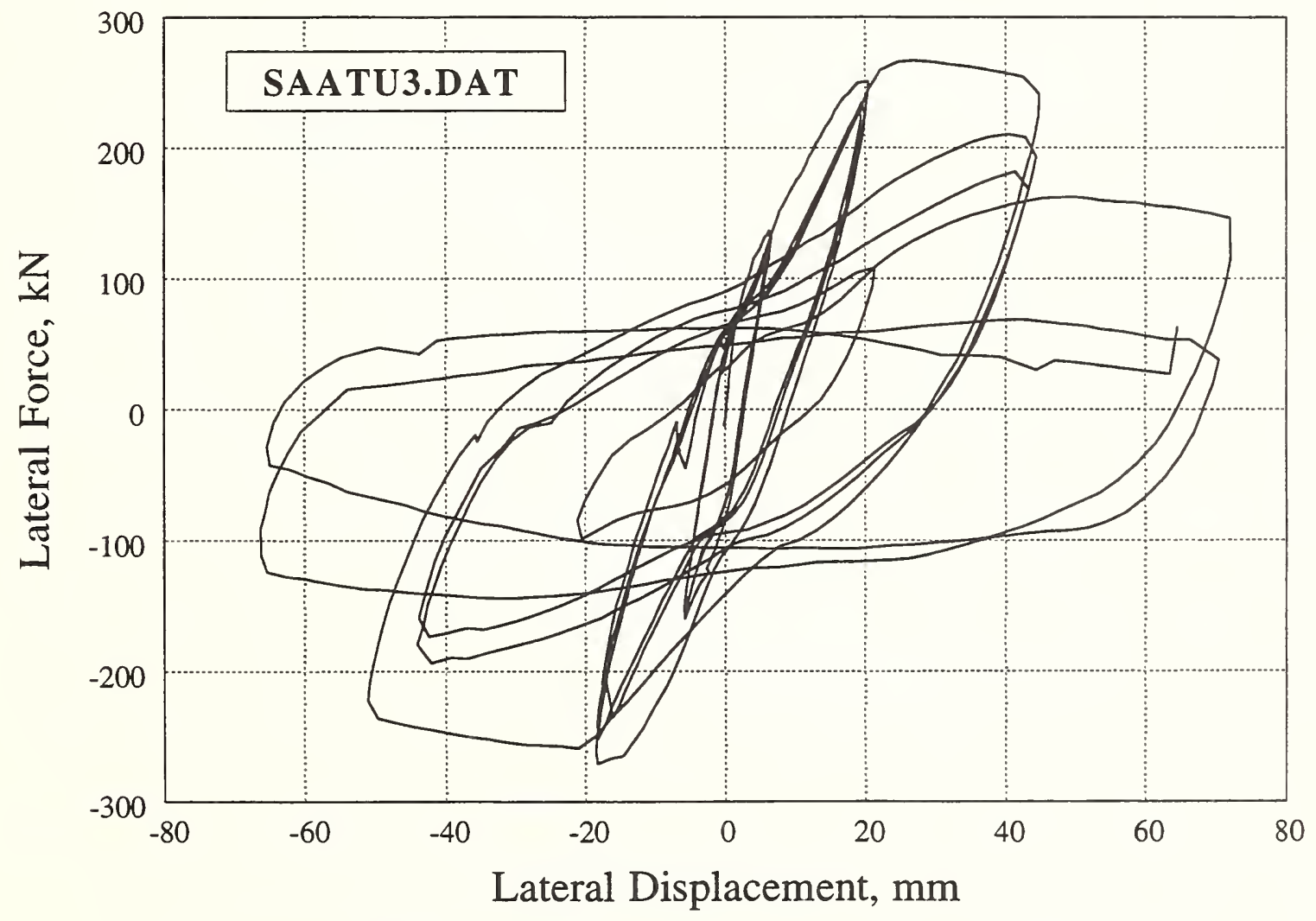

Figure 104. Specimen U3 of Saatcioglu and Ozcebe 1989 


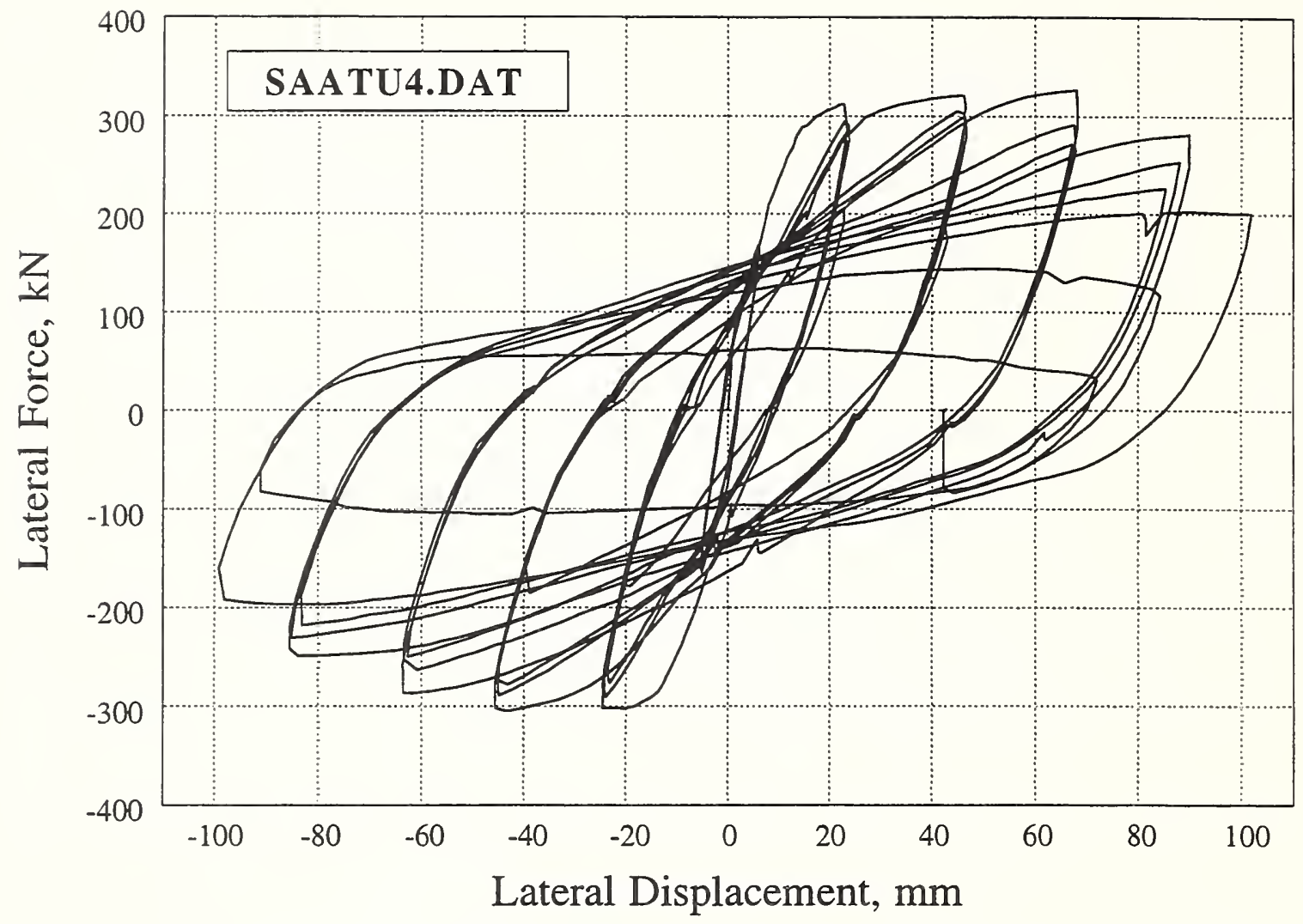

Figure 105. Specimen U4 of Saatcioglu and Ozcebe 1989

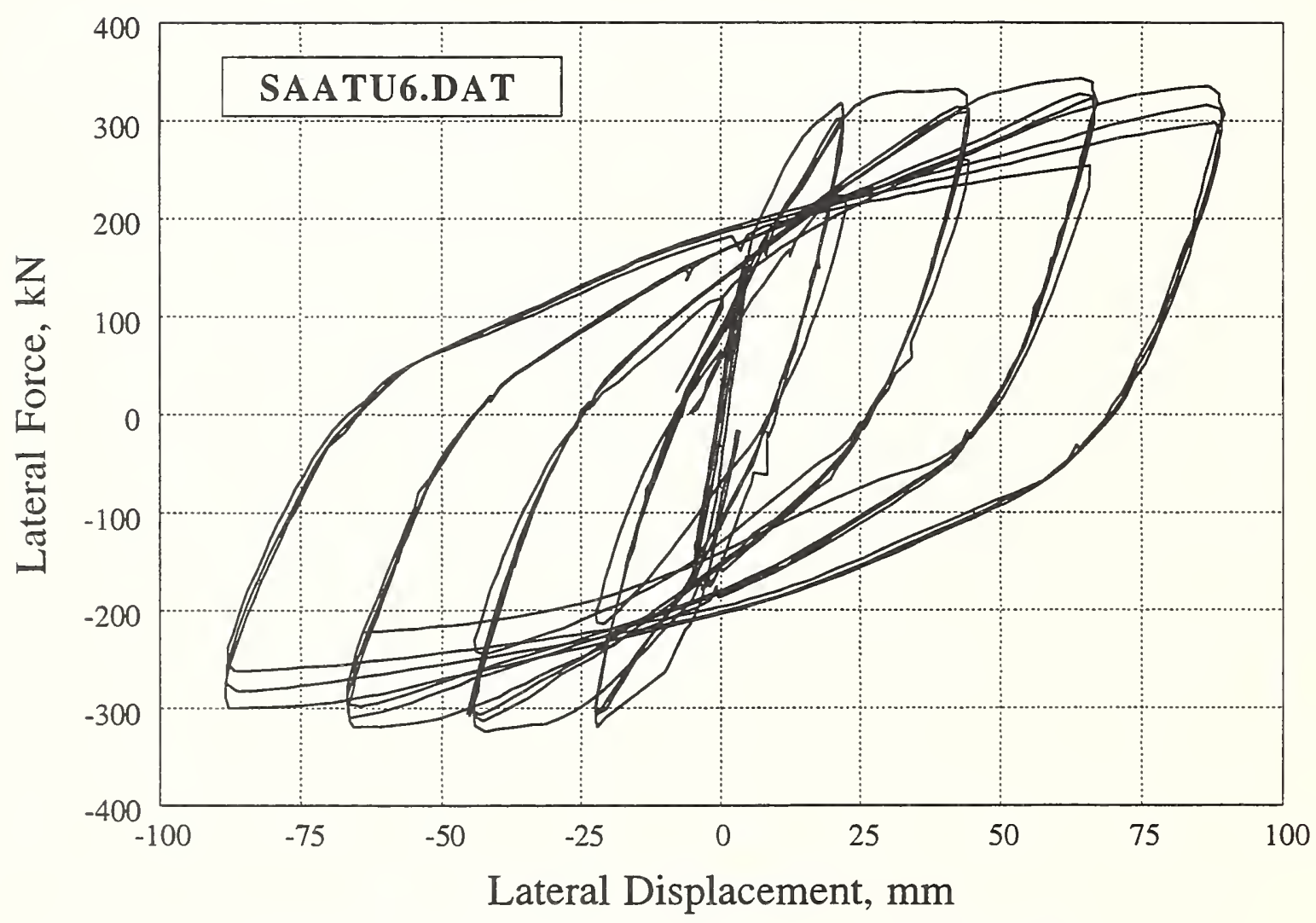

Figure 106. Specimen U6 of Saatcioglu and Ozcebe 1989 


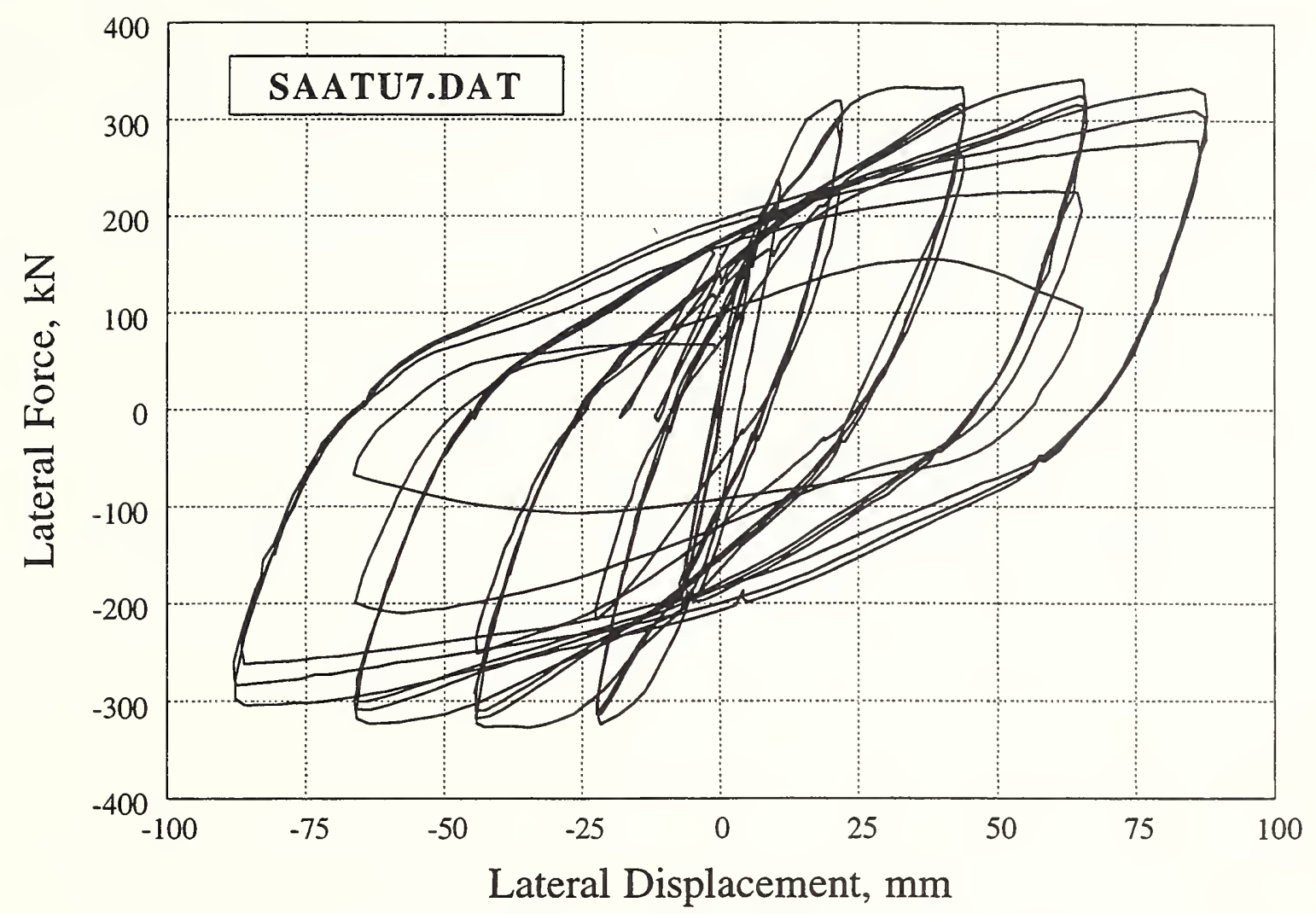

Figure 107. Specimen U7 of Saatcioglu and Ozcebe 1989 
- 96 - 


\section{Chapter 4: Digital Data Files}

Enclosed is one high density (1.44 megabytes) DOS compatible disk. The disk contains 107 digital data files listing lateral deflection-lateral force data, as described in this report.

The files are stored on the disk in a compressed format. To decompress the files, first copy the only file on the disk, RECTCOL.EXE onto the computer hard disk. There should be at least 3 megabytes of disk space available to perform the expansion. Then type the command

\section{RECTCOL.EXE}

and all of the files will be automatically decompressed. The original compressed files, RECTCOL.EXE, may then be erased from the hard disk.

The names of the 107 digital data files correspond to those given in Chapter 2 of the report. All the data files (once they have been de-compressed) are in a format which is readable by common spreadsheet programs. The first line of each file is a short descriptive title. The second line is the total number of deflection-force data pairs, "n", to follow in the data file (i.e., the number of data points in the file). The remaining " $n$ "lines are the deflection-force data pairs, in units of $\mathrm{mm}$ and $\mathrm{kN}$. 


